Supplementary Materials

for

\title{
Intramolecular Hydrogen Atom Transfer Induced 1,2-Migration of Boronate Complexes
}

Dinghai Wang, Kalipada Jana, Armido Studer*

Corresponding author. e-mail: studer@uni-muenster.de

Organisch-Chemisches Institut, Westfälische Wilhelms-Universität, Corrensstraße 40, 48149 Münster, Germany

\section{Content}

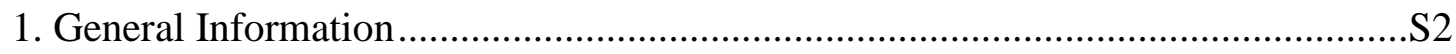

2. General Procedures .......................................................................................S3

2.1 General Procedure 1 for the Intramolecular HAT Reaction from Commercially Available Organolithium Reagents (GP1) ..S3

2.2 General Procedure 2 for the Intramolecular HAT Reaction from Aryl Halides

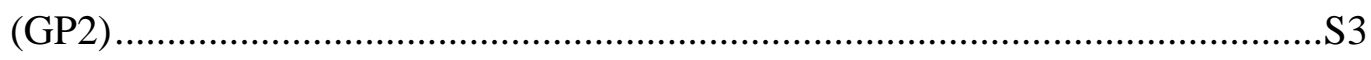

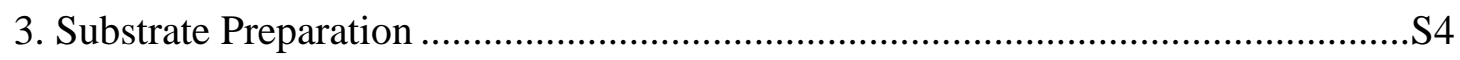

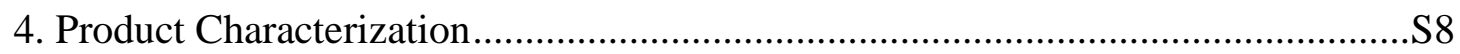

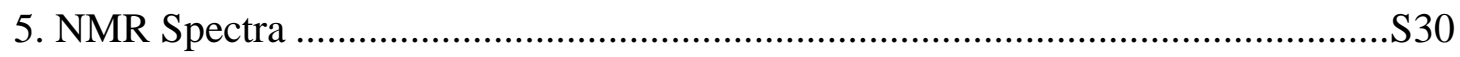




\section{General Information}

All reactions involving air or moisture sensitive reagents were carried out in flamedried glassware under argon atmosphere using standard Schlenk techniques. Solvents used in reactions were either freshly distilled or obtained in extra-dry grade from commercial sources. Diethyl ether $\left(\mathrm{Et}_{2} \mathrm{O}\right)$ was refluxed over $\mathrm{K}$ and freshly distilled from K-Na-alloy (4:1) afterwards. Tetrahydrofuran (THF) was refluxed over Na and distilled from K afterwards. Acetonitrile (MeCN, 99.9\%, Extra Dry over Molecular Sieves) was purchased from Acros Organics. Solvents for extraction and for flash chromatography were distilled. Otherwise noted, commercially available alkyl/aryl lithium reagents were purchased from Acros Organics, Sigma Aldrich and were used as received. All other chemicals were purchased from Fluorochem, ABCR, Acros Organics, Alfa Aesar, Fluka, Sigma Aldrich, TCI and were used as received. Flash chromatography (FC) was performed on Merck silica gel $60(40-63 \mu \mathrm{m})$. Merck silica gel 60 F254 plates were used for thin layer chromatography (TLC) using UV light $(254 / 366 \mathrm{~nm})$ or oxidation with $\mathrm{KMnO} 4$ (1.5 g in $200 \mathrm{~mL}$ H2O, $5 \mathrm{~g} \mathrm{NaHCO} 3$ ) for detection. Melting points (MP) were determined with a Stuart SMP10 and are uncorrected. Infrared spectra (IR) were measured on a Digilab 3100 FT-IR Excalibur Series spectrometer and the position of the absorption bands is given in wave numbers $v\left(\mathrm{~cm}^{-1}\right) .{ }^{\mathbf{1}} \mathbf{H} \mathbf{~ N M R}(300 \mathrm{MHz}, 400 \mathrm{MHz}$ and $600 \mathrm{MHz}),{ }^{13} \mathbf{C ~ N M R}(75 \mathrm{MHz}, 100 \mathrm{MHz}$ and $151 \mathrm{MHz}),{ }^{19} \mathbf{F}$ NMR $(282 \mathrm{MHz}$ and $564 \mathrm{MHz}$ ) and ${ }^{11} \mathbf{B}$ NMR (96 MHz, $128 \mathrm{MHz}$ and $192 \mathrm{MHz}$ ) spectra were measured on a Bruker DPX 300, Bruker AV 300 or an Agilent DD2 600 spectrometer. The multiplicity of all signals were described as s (singlet), d (doublet), t (triplet), q (quartet) and $\mathrm{m}$ (multiplet). Chemical shifts ( $\delta$ in ppm) were referenced on the residual peak of $\mathrm{CDCl}_{3}\left({ }^{1} \mathrm{HNMR}: \delta=7.26 ;{ }^{13} \mathrm{C}\right.$ NMR: $\left.\delta=77.0\right)$ or on an external standard $\left(\mathrm{CFCl}_{3}:{ }^{19} \mathrm{~F}\right.$ NMR: $\delta=0.0)$. HRMS ESI $(\mathrm{m} / \mathrm{z})$ measurements were performed on a Bruker MicroTof and HRMS EI (m/z) on a Waters-Micromass QuattroMicro GC-MS. LED light bulbs $(365 \mathrm{~nm})$ was purchased from LED Engin and fixed on a round module. A borosilicate Schlenk tube was use as the irradiation vessel. No filter for the irradiation was applied. The distance from the light source to the irradiation vessel is about $5 \mathrm{~cm}$ (Figure S1).

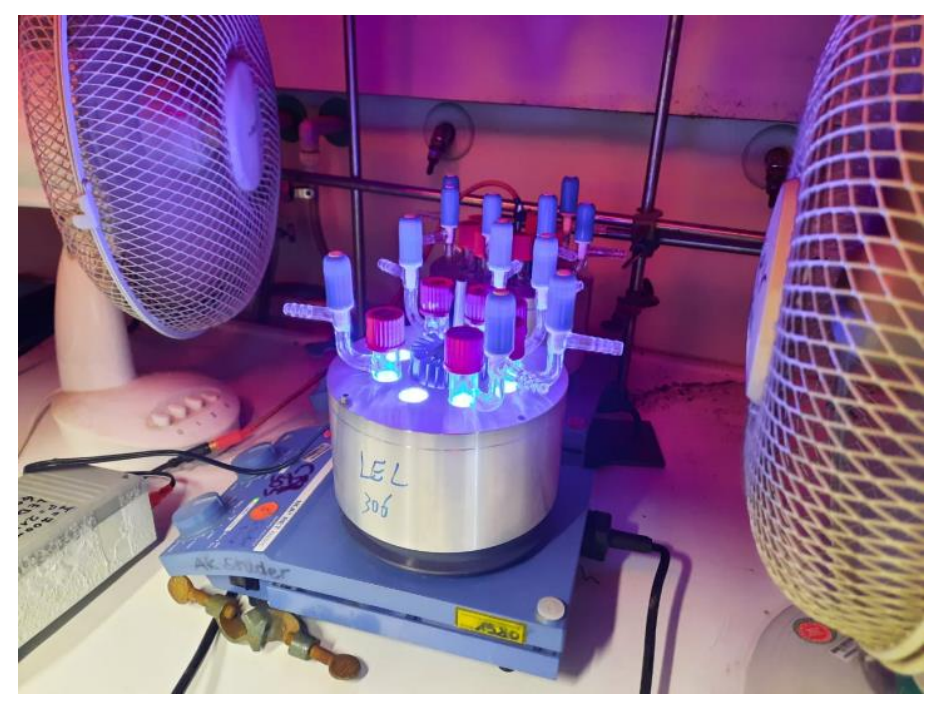

Figure S1. Irradiation system. 


\section{General Procedures}

\subsection{General Procedure 1 for the Intramolecular HAT Reaction from Commercially}

\section{Available Organolithium Reagents (GP1)}

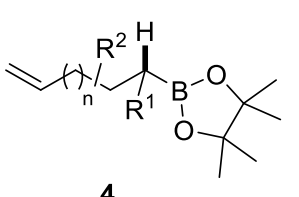

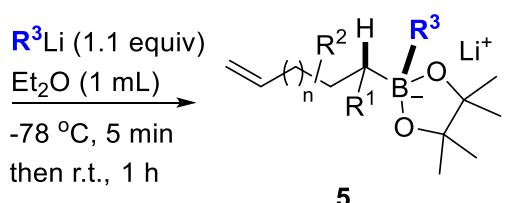

5

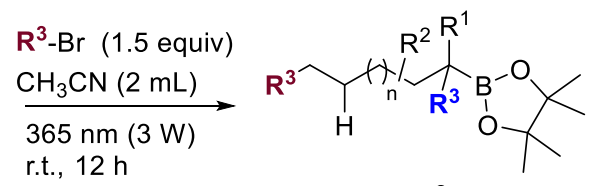

Alkyl boronic ester 4 (0.20 mmol, 1.0 equiv) was dissolved in diethyl ether ( $\mathrm{Et}_{2} \mathrm{O}, 1.0$ $\mathrm{mL})$. The organolithium reagent solution $(0.22 \mathrm{mmol}, 1.1$ equiv) was added dropwise at $-78{ }^{\circ} \mathrm{C}$. The mixture was stirred at $-78{ }^{\circ} \mathrm{C}$ for $5 \mathrm{~min}$ then at room temperature for 1 hour. The solvent was carefully removed in vacuo and further dried for 5 min under high vacuum. After addition of acetonitrile $(2 \mathrm{~mL})$, the mixture was stirred for $1 \mathrm{~min}$ until all solid was dissolved. Then alkyl bromide ( $0.3 \mathrm{mmol}, 1.5$ equiv) was added to the reaction tube. The reaction mixture was irradiated with a $3 \mathrm{~W}$ LED $(365 \mathrm{~nm})$ and stirred at room temperature for $12 \mathrm{~h}$. The reaction mixture was filtered through a pad of silica gel and rinsed with $40 \mathrm{mLEt}_{2} \mathrm{O}$. The organic solvent was removed under reduced pressure. Flash column chromatography eluting with pentane and dichloromethane afforded the desired product 6 .

\subsection{General Procedure 2 for the Intramolecular HAT Reaction from Aryl Halides}

(GP2)

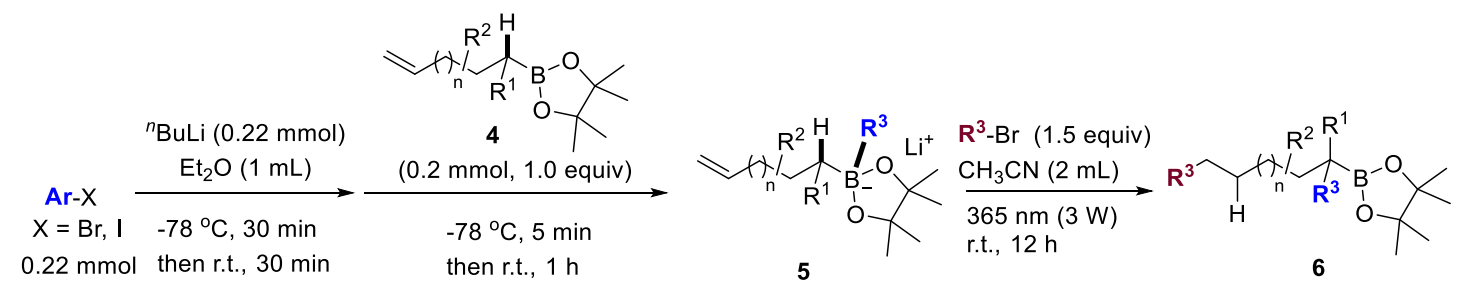

Aryl halide (0.22 mmol, 1.1 equiv) was dissolved in diethyl ether $\left(\mathrm{Et}_{2} \mathrm{O}, 1.0 \mathrm{~mL}\right) . n$ Butyl lithium ( $0.14 \mathrm{~mL}, 0.22 \mathrm{mmol}, 1.1$ equiv, $c=1.6 \mathrm{M}$ in hexane) was added dropwise at $-78{ }^{\circ} \mathrm{C}$. The reaction mixture was stirred at $-78{ }^{\circ} \mathrm{C}$ for $30 \mathrm{~min}$ then at room temperature for $30 \mathrm{~min}$. Alkyl boronic ester 4 ( $0.20 \mathrm{mmol}, 1.0$ equiv) was added to the reaction mixture under argon flow at $-78{ }^{\circ} \mathrm{C}$. The mixture was stirred at $-78{ }^{\circ} \mathrm{C}$ for 5 min then at room temperature for 1 hour. The solvent was carefully removed in vacuo and further dried for $5 \mathrm{~min}$ under high vacuum. After addition of acetonitrile $(2 \mathrm{~mL})$, the mixture was stirred for $1 \mathrm{~min}$ until all solid was dissolved. Then alkyl bromide $(0.3$ mmol, 1.5 equiv) was added to the reaction tube. The reaction mixture was irradiated 
with a $3 \mathrm{~W}$ LED $(365 \mathrm{~nm})$ and stirred at room temperature for $12 \mathrm{~h}$. The reaction mixture was filtered through a pad of silica gel and rinsed with $40 \mathrm{mLEt}_{2} \mathrm{O}$. The organic solvent was removed under reduced pressure. Flash column chromatography eluting with pentane and dichloromethane afforded the desired product 6.

\section{Substrate Preparation}

Preparation of 2-(5,5-dimethylhept-6-en-2-yl)-4,4,5,5-tetramethyl-1,3,2-dioxaborolane (4a)

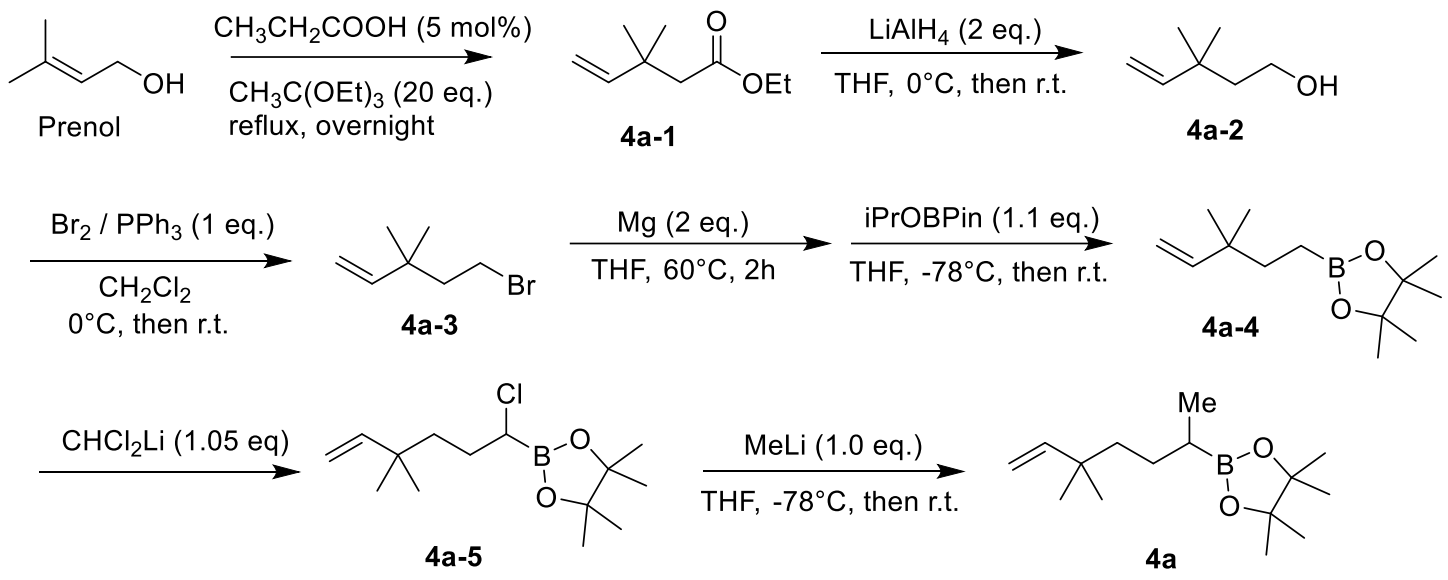

Prenol (5.43 g, $6.4 \mathrm{~mL}, 63.0 \mathrm{mmol})$, propionic acid $(0.23 \mathrm{~g}, 0.23 \mathrm{~mL}, 0.05$ equiv) and triethylorthoacetate $(230 \mathrm{~mL}, 1.26 \mathrm{~mol}, 20$ equiv) were refluxed overnight. The reaction mixture was then poured into a solution of $\mathrm{H}_{2} \mathrm{SO}_{4}(15 \mathrm{~mL})$ and ice water $(300 \mathrm{~mL})$ and stirred overnight. The mixture was extracted with $\mathrm{Et}_{2} \mathrm{O}$. The combined organic layers were washed with $\mathrm{NaOH}$ solution $(10 \% \mathrm{w} / \mathrm{w})$ and brine. After drying over $\mathrm{MgSO}_{4}$ the solvent was evaporated. The resulting mixture was then poured into a solution of $\mathrm{H}_{2} \mathrm{SO}_{4}$ $(15 \mathrm{~mL})$ and ice water $(300 \mathrm{~mL})$ and stirred overnight. The mixture was extracted with $\mathrm{Et}_{2} \mathrm{O}$. The combined organic layers were washed with $\mathrm{NaOH}$ solution $(10 \% \mathrm{w} / \mathrm{w})$ and brine. After drying over $\mathrm{MgSO}_{4}$ solvent was evaporated and 4a-1 was obtained as a colourless liquid (4.35 g, $27.8 \mathrm{mmol}, 44 \%) . \mathrm{R}_{\mathrm{f}}=0.5\left(\mathrm{PE} / \mathrm{Et}_{2} \mathrm{O}=20: 1\right) .{ }^{1} \mathrm{H}$ NMR (300 $\left.\mathrm{MHz}, \mathrm{CDCl}_{3}\right) \delta=5.98-5.86(\mathrm{~m}, 1 \mathrm{H}), 5.03-4.93(\mathrm{~m}, 2 \mathrm{H}), 4.10(\mathrm{q}, J=7.1 \mathrm{~Hz}, 2 \mathrm{H})$, $2.31(\mathrm{~s}, 2 \mathrm{H}), 1.25(\mathrm{t}, J=7.1 \mathrm{~Hz}, 3 \mathrm{H}), 1.15(6 \mathrm{H}, \mathrm{s})$.

Ethyl 3,3-dimethylpent-4-enoate (4a-1, $4.35 \mathrm{~g}, 27.8 \mathrm{mmol})$ was stirred in THF at $0^{\circ} \mathrm{C}$. $\mathrm{LiAlH}_{4}(2.11 \mathrm{~g}, 53.7 \mathrm{mmol}, 2.0$ equiv) was slowly added to the solution. After the addition the solution was stirred for 2 hours and carefully quenched by addition of water (2.1 mL), NaOH-solution ( $4.2 \mathrm{~mL}, 10 \% \mathrm{w} / \mathrm{w})$, and water $(6.3 \mathrm{~mL})$. The white solid was filtered off and the aqueous phase was extracted with $\mathrm{Et}_{2} \mathrm{O}$. After concentration, the product was purified by column chromatography to obtain $\mathbf{4 a - 2}$ a colourless liquid (2.71 $\mathrm{g}, 23.71 \mathrm{mmol}, 85 \%) . \mathrm{R}_{\mathrm{f}}=0.5\left(\mathrm{PE} / \mathrm{Et}_{2} \mathrm{O}=2: 1\right) .{ }^{1} \mathrm{H} \mathrm{NMR}\left(300 \mathrm{MHz}, \mathrm{CDCl}_{3}\right) \delta=5.93$ $-5.81(\mathrm{~m}, 1 \mathrm{H}), 5.02-4.93(\mathrm{~m}, 2 \mathrm{H}), 3.71-3.63(\mathrm{~m}, 2 \mathrm{H}), 1.64(\mathrm{t}, J=7.2 \mathrm{~Hz}, 1 \mathrm{H}), 1.05$ $(\mathrm{s}, 6 \mathrm{H})$. 
Triphenylphosphine (6.21 g, $23.7 \mathrm{mmol}, 1.0$ equiv) was dissolved in dichloromethane. Bromine (3.79 g, $1.21 \mathrm{~mL}, 23.7 \mathrm{mmol}, 1.0$ equiv) was added to the solution. 3,3Dimethylpent-4-en-1-ol (4a-2, $2.71 \mathrm{~g}, 23.7 \mathrm{mmol})$ was added in one portion and the solution was stirred at room temperature over night. The solvent was evaporated, the resulting mixture was stirred in pentane and filtered. The filtrate was further purified by column chromatography to afford $\mathbf{4 a - 3}$ as a colourless liquid (4.74 g, $17.2 \mathrm{mmol}$, $72 \%) . \mathrm{R}_{\mathrm{f}}=0.7(\mathrm{PE}) .{ }^{1} \mathrm{H}$ NMR $\left(300 \mathrm{MHz}, \mathrm{CDCl}_{3}\right) \delta=5.82-5.70(\mathrm{~m}, 1 \mathrm{H}), 5.04-4.93$ $(\mathrm{m}, 2 \mathrm{H}), 3.36-3.28(\mathrm{~m}, 2 \mathrm{H}), 1.90(\mathrm{~m}, 2 \mathrm{H}), 1.04(\mathrm{~s}, 6 \mathrm{H})$.

5-Bromo-3,3-dimethylpent-1-ene (4a-3, $4.74 \mathrm{~g}, 17.2 \mathrm{mmol})$ was added to a suspension of magnesium chippings ( $0.83 \mathrm{~g}, 34.3 \mathrm{mmol}, 2.0$ equiv) in dry THF with a small piece of iodine. After stirring the solution at $60^{\circ} \mathrm{C}$ for 2 hours it was cooled down to $-78{ }^{\circ} \mathrm{C}$ and 2-isoprpoxy-4,4,5,5-tetramethyl-1,3,2-dioxaborolane (3.51 g, $3.86 \mathrm{~mL}, 17.2 \mathrm{mmol}$, 1.0 equiv) was added in one portion. The reaction mixture was stirred overnight, quenched with $\mathrm{H}_{2} \mathrm{O}$, and extracted with $\mathrm{Et}_{2} \mathrm{O}$. After further purification by column chromatography, the product $\mathbf{4 a - 4}$ was obtained as a colourless liquid (1.38 g, $6.2 \mathrm{mmol}$, $36 \%) . \mathrm{R}_{\mathrm{f}}=0.5\left(\mathrm{PE} / \mathrm{Et}_{2} \mathrm{O}=10: 1\right) .{ }^{1} \mathrm{H}$ NMR $(300 \mathrm{MHz}$, Chloroform- $d) \delta 5.84-5.63(\mathrm{~m}$, $1 \mathrm{H}), 5.00-4.79(\mathrm{~m}, 2 \mathrm{H}), 1.43-1.34(\mathrm{~m}, 2 \mathrm{H}), 1.24(\mathrm{~s}, 12 \mathrm{H}), 0.95(\mathrm{~s}, 6 \mathrm{H}), 0.74-0.60$ (m, 2H). ${ }^{11} \mathrm{~B}$ NMR $\left(96 \mathrm{MHz}, \mathrm{CDCl}_{3}\right) \delta=33.9$.

Dichloromethane $(0.81 \mathrm{~g}, 0.61 \mathrm{~mL}, 9.6 \mathrm{mmol}, 1.55$ equiv) was diluted in THF (30 mL) and cooled down to $-100{ }^{\circ} \mathrm{C}$ in a $95 \%$ ethanol/liquid nitrogen slush bath. $\mathrm{n}$ Butyllithium-solution (4.1 mL, 1.6 M in Hexane, $6.5 \mathrm{mmol}, 1.05$ equiv) was carefully added to the solution over 10 minutes. After 20 minutes, the 2-(3,3-dimethylpent-4-en1-yl)-4,4,5,5-tetramethyl-1,3,2-dioxaborolane (1.38 g, $6.2 \mathrm{mmol})$ was added in one portion. The mixture was allowed to warm to room temperature in the slush bath overnight and was stirred throughout this time. After that, the solvent was evaporated and the resulting mixture was stirred in pentane to precipitate the lithium chloride. The precipitate was filtered off to obtain $\mathbf{4 a - 5}$ as a colourless liquid (1.67 g, $6.1 \mathrm{mmol}, 99 \%)$. ${ }^{1} \mathrm{H}$ NMR $\left(300 \mathrm{MHz}, \mathrm{CDCl}_{3}\right) \delta=5.74-5.62(\mathrm{~m}, 1 \mathrm{H}), 4.89-4.80(\mathrm{~m}, 2 \mathrm{H}), 3.33-3.24$ (m, 1H), $1.75-1.59(\mathrm{~m}, 2 \mathrm{H}), 1.51-1.37(\mathrm{~m}, 2 \mathrm{H}), 1.22(\mathrm{~s}, 12 \mathrm{H}), 0.91(\mathrm{~s}, 6 \mathrm{H}) .4 \mathbf{a}-5$ was directly used for the next step without further purification.

2-(1-Chloro-4,4-dimethylhex-5-en-1-yl)-4,4,5,5-tetramethyl-1,3,2-dioxaborolane (4a5) $(0.27 \mathrm{~g}, 1 \mathrm{mmol})$ was diluted in $\mathrm{THF}(3 \mathrm{~mL})$ and cooled down to $-78{ }^{\circ} \mathrm{C}$. Methyllithium solution ( $0.63 \mathrm{~mL}, 1.6 \mathrm{M}$ in $\mathrm{Et}_{2} \mathrm{O}, 1 \mathrm{mmol}, 1.0$ equiv) was added dropwise to the solution. This reaction mixture was allowed to warm up to room temperature and was stirred overnight. The solvent was evaporated and the product was purified by column chromatography to afford 4a a colourless liquid $(0.1218 \mathrm{~g}, 0.483$ mmol, $48 \%) . \mathrm{R}_{\mathrm{f}}=0.5\left(\mathrm{PE} / \mathrm{Et}_{2} \mathrm{O}=20: 1\right) .{ }^{1} \mathrm{H}$ NMR $(300 \mathrm{MHz}$, Chloroform- $d) \delta 5.83-$ $5.69(\mathrm{~m}, 1 \mathrm{H}), 4.96-4.82(\mathrm{~m}, 2 \mathrm{H}), 1.40-1.15(\mathrm{~m}, 16 \mathrm{H}), 1.03-0.88(\mathrm{~m}, 10 \mathrm{H}) .{ }^{13} \mathrm{C}$ NMR $\left(75 \mathrm{MHz}, \mathrm{CDCl}_{3}\right) \delta 148.8,110.0,82.8,42.0,36.5,28.0,26.8,26.7,24.8,24.7$, 15.6, carbon attached to boron atom not observed. ${ }^{11} \mathrm{~B} \mathrm{NMR}\left(96 \mathrm{MHz}, \mathrm{CDCl}_{3}\right) \delta 34.4$. 
2-(4,4-Dimethylhex-5-en-1-yl)-4,4,5,5-tetramethyl-1,3,2-dioxaborolane (4z)<smiles>C=CC(C)(C)CCB1OC(C)(C)C(C)(C)O1</smiles>

$4 a-4$

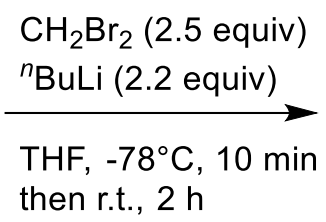

then r.t., $2 \mathrm{~h}$

2-(2,2-Dimethylpent-4-en-1-yl)-4,4,5,5-tetramethyl-1,3,2-dioxaborolane (4a-4, 67.2 $\mathrm{mg}, 0.3 \mathrm{mmol})$ and dibromomethane $(52.7 \mu \mathrm{L}, 130.5 \mathrm{mg}, 0.75 \mathrm{mmol}, 2.5$ equiv) were dissolved in THF ( $9 \mathrm{~mL}$ ). The solution was cooled to $-78{ }^{\circ} \mathrm{C}$ and ${ }^{n} \mathrm{BuLi}(0.41 \mathrm{~mL}, 1.6$ $\mathrm{M}$ in hexane, $0.66 \mathrm{mmol}, 2.2$ equiv) was added dropwise. The solution was stirred at $78{ }^{\circ} \mathrm{C}$ for $10 \mathrm{~min}$ then at room temperature for another 2 hours. The solvent was evaporated and the product was purified by column chromatography to afford $\mathbf{4 z}$ as a colorless liquid (39.3 mg, 55\%). $\mathrm{R}_{\mathrm{f}}=0.4(\mathrm{PE} / \mathrm{DCM}=3: 2) .{ }^{1} \mathrm{H}$ NMR (300 MHz, Chloroform- $d$ ) $\delta 5.83-5.64(\mathrm{~m}, 1 \mathrm{H}), 4.96-4.80(\mathrm{~m}, 2 \mathrm{H}), 1.39-1.15(\mathrm{~m}, 16 \mathrm{H}), 0.95$ $(\mathrm{s}, 6 \mathrm{H}), 0.71(\mathrm{t}, J=7.0 \mathrm{~Hz}, 2 \mathrm{H}) .{ }^{13} \mathrm{C} \mathrm{NMR}\left(76 \mathrm{MHz}, \mathrm{CDCl}_{3}\right) \delta 148.7,109.9,82.8,45.8$, 36.6, 26.7, 24.8, 18.9, carbon attached to boron atom not observed. ${ }^{10} \mathrm{~B} \mathrm{NMR}(32 \mathrm{MHz}$, $\left.\mathrm{CDCl}_{3}\right) \delta$ 32.7. HRMS (ESI) $m / z$ : $[\mathrm{M}+\mathrm{Na}]^{+}$calcd. for $\mathrm{C}_{14} \mathrm{H}_{27} \mathrm{O}_{2} \mathrm{BNa}^{+} 261.1999$; found 261.1994.

2-(6,6-Dimethyloct-7-en-3-yl)-4,4,5,5-tetramethyl-1,3,2-dioxaborolane (4aa)<smiles>C=CC(C)(C)CCC(Cl)B1OC(C)(C)C(C)(C)O1</smiles>

4a-5

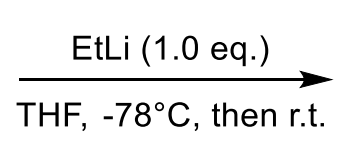

THF, $-78^{\circ} \mathrm{C}$, then r.t.<smiles>C=CC(C)(C)CCC(CC)B1OC(C)(C)C(C)(C)O1</smiles>

4aa

2-(1-Chloro-4,4-dimethylhex-5-en-1-yl)-4,4,5,5-tetramethyl-1,3,2-dioxaborolane (4a5) $(0.27 \mathrm{~g}, 1.0 \mathrm{mmol})$ was diluted in THF $(3 \mathrm{~mL})$ and cooled down to $-78{ }^{\circ} \mathrm{C}$. Ethyllithium-solution ( $2.0 \mathrm{~mL}, 0.5 \mathrm{M}$ in benzene, $1 \mathrm{mmol}, 1.0$ equiv) was added dropwise to the solution. This reaction mixture was allowed to warm up to room temperature and was stirred overnight. The solvent was evaporated and the product was purified by column chromatography to afford 4aa as a colourless liquid $(0.0912 \mathrm{~g}, 0.343$ mmol, 34\%). $\mathrm{R}_{\mathrm{f}}=0.5\left(\mathrm{PE} / \mathrm{Et}_{2} \mathrm{O}=20: 1\right) .{ }^{1} \mathrm{H}$ NMR $(300 \mathrm{MHz}$, Chloroform- $d) \delta 5.83-$ $5.67(\mathrm{~m}, 1 \mathrm{H}), 4.95-4.82(\mathrm{~m}, 2 \mathrm{H}), 1.51-1.31(\mathrm{~m}, 2 \mathrm{H}), 1.30-1.19(\mathrm{~m}, 16 \mathrm{H}), 0.96(\mathrm{~s}$, $6 \mathrm{H}), 0.88(\mathrm{t}, J=7.4 \mathrm{~Hz}, 3 \mathrm{H}), 0.83-0.74(\mathrm{~m}, 1 \mathrm{H}) .{ }^{13} \mathrm{C} \mathrm{NMR}\left(76 \mathrm{MHz}, \mathrm{CDCl}_{3}\right) \delta 148.8$, $110.1,82.9,42.2,36.6,26.9,26.7,26.0,24.9,24.9,24.4,13.9$, carbon attached to boron atom not observed. ${ }^{11} \mathrm{~B} \mathrm{NMR}\left(96 \mathrm{MHz}, \mathrm{CDCl}_{3}\right) \delta 34.2$. FTIR (neat): $v\left(\mathrm{~cm}^{-1}\right) 2958$, 2929, 2868, 1640, 1463, 1413, 1388, 1315, 1262, 1213, 1144, 1112, 1000, 968, 908, 856.

4,4,5,5-Tetramethyl-2-(2,2,6,6-tetramethyloct-7-en-3-yl)-1,3,2-dioxaborolane (4ab) 


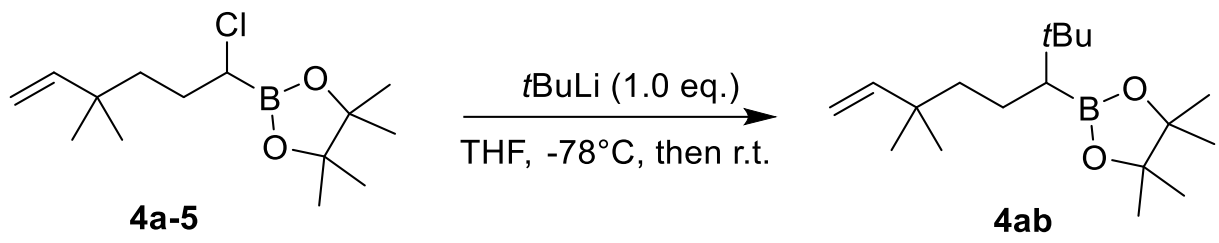

2-(1-Chloro-4,4-dimethylhex-5-en-1-yl)-4,4,5,5-tetramethyl-1,3,2-dioxaborolane (4a$5,0.27 \mathrm{~g}, 1.0 \mathrm{mmol})$ was diluted in THF $(3 \mathrm{~mL})$ and cooled down to $-78^{\circ} \mathrm{C}$. tert-Butyllithium solution $(0.59 \mathrm{~mL}, 1.7 \mathrm{M}$ in Pentane, $1.0 \mathrm{mmol}, 1.0$ equiv) was added dropwise to the solution. This reaction mixture was allowed to warm to room temperature and was stirred overnight. The solvent was evaporated, and the product was purified by column chromatography to afford $\mathbf{4 a b}$ a colourless liquid $(0.25 \mathrm{~g}, 0.85$ mmol, 85\%). ${ }^{1} \mathrm{H}$ NMR (300 MHz, Chloroform- $d$ ) $\delta 5.86-5.67(\mathrm{~m}, 1 \mathrm{H}), 4.99-4.81$ $(\mathrm{m}, 2 \mathrm{H}), 1.37-1.11(\mathrm{~m}, 16 \mathrm{H}), 0.96(\mathrm{~s}, 6 \mathrm{H}), 0.92(\mathrm{~s}, 9 \mathrm{H}) .{ }^{13} \mathrm{C} \mathrm{NMR}\left(76 \mathrm{MHz}, \mathrm{CDCl}_{3}\right)$ $\delta$ 148.7, 110.0, 82.8, 43.4, 36.8, 32.1, 29.6, 27.0, 26.6, 25.1, 24.9, 21.8, carbon attached to boron atom not observed. ${ }^{11} \mathrm{~B} \mathrm{NMR}\left(96 \mathrm{MHz}, \mathrm{CDCl}_{3}\right) \delta 33.8$. FTIR (neat): $v\left(\mathrm{~cm}^{-1}\right)$ 2959, 2869, 1639, 1474, 1371, 1314, 1262, 1143, 1104, 1000, 967, 967, 908, 866, 844, 775,688 .

2-(5,5-Dimethyloct-7-en-2-yl)-4,4,5,5-tetramethyl-1,3,2-dioxaborolane (4ac)

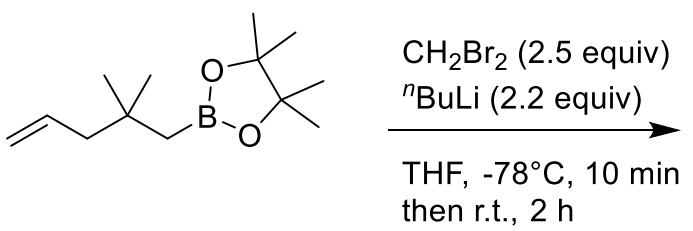

4ac-1<smiles>C=CCC(C)(C)CCC(Cl)B1OC(C)(C)C(C)(C)O1</smiles>

4ac-3
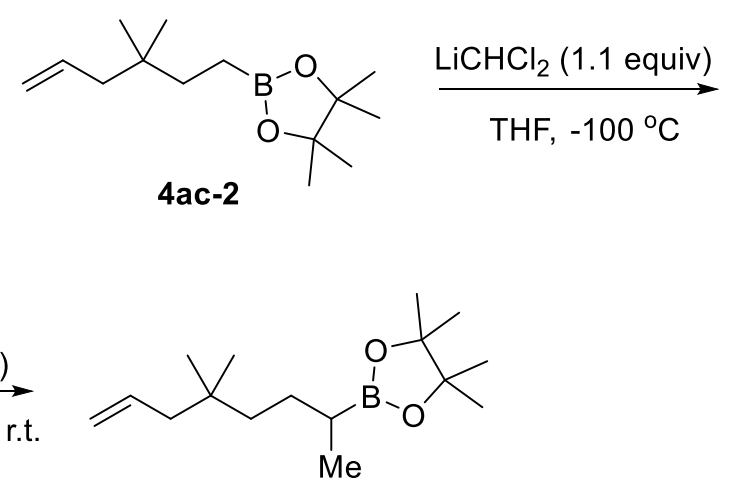

$4 a c$

2-(2,2-Dimethylpent-4-en-1-yl)-4,4,5,5-tetramethyl-1,3,2-dioxaborolane (4ac-1, 201.6 $\mathrm{mg}, 0.9 \mathrm{mmol})$ and dibromomethane $(158.1 \mu \mathrm{L}, 334.5 \mathrm{mg}, 1.98 \mathrm{mmol}, 2.2$ equiv) were dissolved in THF ( $9 \mathrm{~mL})$. The solution was cooled to $-78^{\circ} \mathrm{C}$ and $\mathrm{nBuLi}(1.23 \mathrm{~mL}, 1.6$ $\mathrm{M}$ in hexane, $2.25 \mathrm{mmol}, 2.5$ equiv) was added dropwise. The solution was stirred at $78{ }^{\circ} \mathrm{C}$ for $10 \mathrm{~min}$ then at room temperature for another 2 hours. The solvent was evaporated and the product was purified by column chromatography to afford 4ac-2 a colourless liquid $(116.5 \mathrm{mg}, 54 \%) . \mathrm{R}_{\mathrm{f}}=0.5(\mathrm{PE} / \mathrm{DCM}=3: 2) .{ }^{1} \mathrm{H}$ NMR $(400 \mathrm{MHz}$, Chloroform- $d) \delta 5.89-5.71(\mathrm{~m}, 1 \mathrm{H}), 5.03-4.90(\mathrm{~m}, 2 \mathrm{H}), 1.97-1.87(\mathrm{~m}, 2 \mathrm{H}), 1.34-$ $1.26(\mathrm{~m}, 2 \mathrm{H}), 1.24(\mathrm{~s}, 12 \mathrm{H}), 0.81(\mathrm{~s}, 6 \mathrm{H}), 0.75-0.65(\mathrm{~m}, 2 \mathrm{H}) .{ }^{13} \mathrm{C} \mathrm{NMR}(101 \mathrm{MHz}$, $\left.\mathrm{CDCl}_{3}\right) \delta 136.0,116.4,82.9,45.9,35.7,33.5,26.3,24.8$, carbon attached to boron atom not observed. ${ }^{11} \mathrm{~B}$ NMR $\left(128 \mathrm{MHz}, \mathrm{CDCl}_{3}\right) \delta 34.2$. HRMS (ESI) $\mathrm{m} / z:[\mathrm{M}+\mathrm{Na}]^{+}$calcd. for $\mathrm{C}_{14} \mathrm{H}_{27} \mathrm{O}_{2} \mathrm{BNa}^{+}$261.1999; found 261.1994. FTIR (neat): $v\left(\mathrm{~cm}^{-1}\right)$ 2978, 2959, 2930, 1639, 1469, 1370, 1329, 1314, 1273, 1215, 1145, 1108, 995, 968, 910, 887, 848, 739, 
674.

Dichloromethane $(0.1 \mathrm{~mL})$ was dissolved in THF $(1 \mathrm{~mL})$ and cooled down to $-100{ }^{\circ} \mathrm{C}$ in a $95 \%$ ethanol/liquid nitrogen slush bath. n-Butyllithium solution $(0.2 \mathrm{~mL}, 1.6 \mathrm{M}$ in Hexane, 0.33 mmol, 1.1 equiv) was carefully added to the solution. After 20 minutes, 2-(3,3-Dimethylhex-5-en-1-yl)-4,4,5,5-tetramethyl-1,3,2-dioxaborolane (4ac-2, 71.4 $\mathrm{mg}, 0.3 \mathrm{mmol}$ ) was added in one portion. The mixture was allowed to warm to room temperature in the slush bath overnight and was stirred throughout this time. After that, the solvent was evaporated and the resulting mixture was stirred in pentane to precipitate the lithium chloride. The precipitate was filtered off to obtain $\mathbf{4 a - 3}$ as a colourless liquid and used for the next step without further purification.

4a-3 was dissolved in $\mathrm{Et}_{2} \mathrm{O}(1 \mathrm{~mL})$ and the solution was cooled to $-78^{\circ} \mathrm{C}$. Methyllithium solution $(0.20 \mathrm{~mL}, 1.6 \mathrm{M}$ in Et2O, $0.32 \mathrm{mmol}, 1.1$ equiv) was added to dropwise to the solution. This reaction mixture was allowed to warm to room temperature and was stirred overnight. The solvent was evaporated, and the product was purified by column chromatography to afford 4ac a colourless liquid (19.0 mg, 24\% yield for two steps). ${ }^{1} \mathrm{H}$ NMR (300 MHz, Chloroform- $d$ ) $\delta 5.89-5.61(\mathrm{~m}, 1 \mathrm{H}), 5.08-4.78(\mathrm{~m}, 2 \mathrm{H}), 2.04-$ $1.71(\mathrm{~m}, 2 \mathrm{H}), 1.40-1.04(\mathrm{~m}, 16 \mathrm{H}), 0.89(\mathrm{~s}, 3 \mathrm{H}), 0.77(\mathrm{~s}, 6 \mathrm{H}) .{ }^{13} \mathrm{C} \mathrm{NMR}(76 \mathrm{MHz}$, $\left.\mathrm{CDCl}_{3}\right) \delta 136.0,116.4,82.8,46.4,41.2,33.1,27.5,27.0,26.9,24.8,24.7,15.6$, carbon attached to boron atom not observed. HRMS (ESI) $\mathrm{m} / z$ : $[\mathrm{M}+\mathrm{Na}]^{+}$calcd. for $\mathrm{C}_{14} \mathrm{H}_{27} \mathrm{O}_{2} \mathrm{BNa}^{+}$289.2312; found 289.2309.

\section{Product Characterization}

4,4,5,5-Tetramethyl-2-(11,11,12,12,13,13,14,14,15,15,16,16,16-tridecafluoro-5,8,8trimethylhexadecan-5-yl)-1,3,2-dioxaborolane (6a)

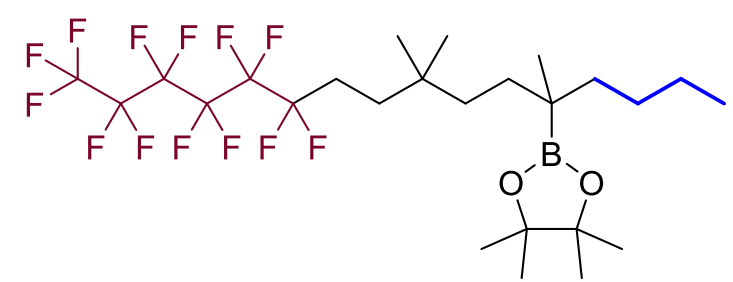

Following GP1 using 2-(5,5-dimethylhept-6-en-2-yl)-4,4,5,5-tetramethyl-1,3,2dioxaborolane (4a, $60 \mu \mathrm{L}, 50.4 \mathrm{mg}, 0.20 \mathrm{mmol}), n$-butyl lithium $(0.14 \mathrm{~mL}, 0.22 \mathrm{mmol}$, 1.1 equiv, $c=1.6 \mathrm{M}$ in hexane) and 1-bromoperfluorohexane $(64 \mu \mathrm{L}, 119.7 \mathrm{mg}, 0.3$ mmol), flash chromatography eluting with pentane/DCM $(5: 1)$ afforded $\mathbf{6 a}$ as a light yellow sticky oil $\left(82.4 \mathrm{mg}, 66 \%\right.$ yield). $\mathrm{R}_{\mathrm{f}} 0.4$ (pentane/DCM = 5:1). ${ }^{1} \mathrm{H}$ NMR (300 $\mathrm{MHz}$, Chloroform- $d$ ) $\delta 2.16-1.85(\mathrm{~m}, 2 \mathrm{H}), 1.49-1.43(\mathrm{~m}, 2 \mathrm{H}), 1.40-1.05(\mathrm{~m}, 10 \mathrm{H})$, $1.22(\mathrm{~s}, 12 \mathrm{H}), 0.92-0.84(\mathrm{~m}, 12 \mathrm{H}) .{ }^{13} \mathrm{C}\left\{{ }^{1} \mathrm{H},{ }^{19} \mathrm{~F}\right\}$ NMR $(151 \mathrm{MHz}$, Chloroform- $d) \delta$ 119.0, 117.3, 111.2, 111.1, 110.3, 108.5, 82.9, 39.2, 37.2, 33.1, 32.0, 30.9, 27.8, 27.0, $26.9,26.1,24.8,23.6,21.3,14.1$, carbon attached to boron atom not observed. ${ }^{19} \mathrm{~F}\left\{{ }^{1} \mathrm{H}\right\}$ NMR (282 MHz, Chloroform- $d$ ) $\delta-80.90(\mathrm{tt}, J=10.0,2.6 \mathrm{~Hz}),-114.3--114.5(\mathrm{~m})$, $121.8--122.1(\mathrm{~m}),-122.7--123.1(\mathrm{~m}),-123.2--123.4(\mathrm{~m}),-126.1--126.3(\mathrm{~m}) .{ }^{11} \mathrm{~B}$ NMR (192 MHz, Chloroform- $d$ ) $\delta 34.8$. HRMS (ESI) $m / z:[\mathrm{M}+\mathrm{Na}]^{+}$calcd. for $\mathrm{C}_{25} \mathrm{H}_{38}$ 
$\mathrm{O}_{2} \mathrm{BF}_{13} \mathrm{Na}^{+}$651.2654; found 651.2654. FTIR (neat): $v\left(\mathrm{~cm}^{-1}\right)$ 2961, 2933, 1471, 1372, 1306, 1238, 1206, 1166, 1144, 908, 855, 811, 733, 694, 651.

4,4,5,5-Tetramethyl-2-(8,8,9,9,10,10,11,11,12,12,13,13,13-tridecafluoro-2,5,5trimethyltridecan-2-yl)-1,3,2-dioxaborolane (6b)

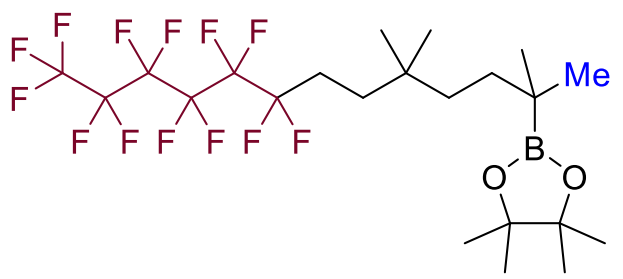

Following GP1 using 2-(5,5-dimethylhept-6-en-2-yl)-4,4,5,5-tetramethyl-1,3,2dioxaborolane (4a, $60 \mu \mathrm{L}, 50.4 \mathrm{mg}, 0.20 \mathrm{mmol})$, methyl lithium $(0.14 \mathrm{~mL}, 0.22 \mathrm{mmol}$, 1.1 equiv, $\left.c=1.6 \mathrm{M}^{\text {in }} \mathrm{Et}_{2} \mathrm{O}\right)$ and 1-bromoperfluorohexane $(64 \mu \mathrm{L}, 119.7 \mathrm{mg}, 0.3 \mathrm{mmol})$, flash chromatography eluting with pentane/DCM (4:1) afforded $\mathbf{6 b}$ as a yellow sticky oil (74.2 mg, 63\% yield). $\mathrm{R}_{\mathrm{f}} 0.4$ (pentane/DCM $\left.=4: 1\right) .{ }^{1} \mathrm{H}$ NMR $(300 \mathrm{MHz}$, Chloroform- $d$ ) $\delta 2.16-1.85(\mathrm{~m}, 2 \mathrm{H}), 1.55-1.40(\mathrm{~m}, 2 \mathrm{H}), 1.21(\mathrm{~s}, 12 \mathrm{H}), 1.19-1.07$ $(\mathrm{m}, 4 \mathrm{H}), 0.91(\mathrm{~s}, 6 \mathrm{H}), 0.87(\mathrm{~s}, 6 \mathrm{H}) .{ }^{13} \mathrm{C}\left\{{ }^{1} \mathrm{H},{ }^{19} \mathrm{~F}\right\}$ NMR $(126 \mathrm{MHz}$, Chloroform- $d) \delta$ 119.0, 117.3, 111.2, 111.1, 110.3, 108.5, 82.9, 37.9, 35.0, 31.9, 30.9, 27.0, 26.1, 25.0, 24.6, carbon attached to boron atom not observed. ${ }^{19} \mathrm{~F}\left\{{ }^{1} \mathrm{H}\right\}$ NMR $(470 \mathrm{MHz}$, Chloroform- $d$ ) $\delta-80.27--82.16(\mathrm{~m}),-113.65--115.23(\mathrm{~m}),-121.46--122.31(\mathrm{~m}),-$ $122.82--123.04(\mathrm{~m}),-123.2--123.4 \mathrm{~m}),-126.1--126.3(\mathrm{~m}) .{ }^{11} \mathrm{~B}$ NMR $(96 \mathrm{MHz}$, Chloroform- $d$ ) $\delta$ 35.3. HRMS (ESI) $m / z:[\mathrm{M}+\mathrm{Na}]^{+}$calcd. for $\mathrm{C}_{22} \mathrm{H}_{32} \mathrm{O}_{2} \mathrm{BF}_{13} \mathrm{Na}^{+}$ 609.2184, found 609.2184. FTIR (neat): $v\left(\mathrm{~cm}^{-1}\right)$ 2961, 2865, 1477, 1368, 1309, 1237 , 1193, 1143, 1075, 1022, 967, 853, 807, 709, 692.

4,4,5,5-Tetramethyl-2-(13,13,14,14,15,15,16,16,17,17,18,18,18-tridecafluoro-7,10,10trimethyloctadecan-7-yl)-1,3,2-dioxaborolane (6c)

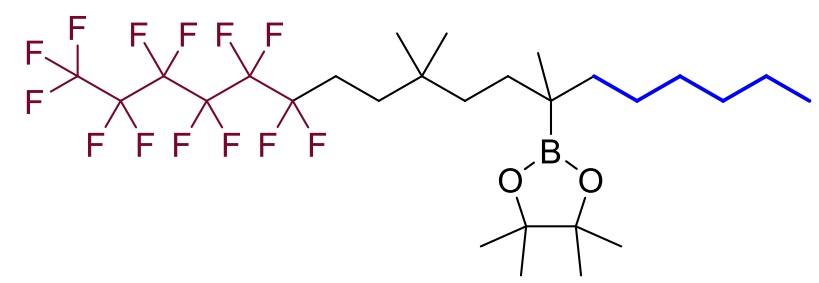

Following GP1 using 2-(5,5-dimethylhept-6-en-2-yl)-4,4,5,5-tetramethyl-1,3,2dioxaborolane (4a, $60 \mu \mathrm{L}, 50.4 \mathrm{mg}, 0.20 \mathrm{mmol}), n$-hexyl lithium $(0.09 \mathrm{~mL}, 0.22 \mathrm{mmol}$, 1.1 equiv, $c=2.5 \mathrm{M}$ in hexane) and 1-bromoperfluorohexane ( $64 \mu \mathrm{L}, 119.7 \mathrm{mg}, 0.3$ mmol), flash chromatography eluting with pentane/DCM (5:1) afforded $\mathbf{6 c}$ as light yellow sticky oil $\left(79.8 \mathrm{mg}, 61 \%\right.$ yield). $\mathrm{R}_{\mathrm{f}} 0.7$ (pentane/DCM $\left.=4: 1\right) .{ }^{1} \mathrm{H}$ NMR $(300$ $\mathrm{MHz}$, Chloroform- $d$ ) $\delta 2.15-1.86(\mathrm{~m}, 2 \mathrm{H}), 1.50-1.42(\mathrm{~m}, 2 \mathrm{H}), 1.40-1.05(\mathrm{~m}, 14 \mathrm{H})$, $1.22(\mathrm{~s}, 12 \mathrm{H}), 0.90-0.83(\mathrm{~m}, 12 \mathrm{H}) .{ }^{13} \mathrm{C}\left\{{ }^{1} \mathrm{H},{ }^{19} \mathrm{~F}\right\}$ NMR $(126 \mathrm{MHz}$, Chloroform- $d) \delta$ 119.0, 117.3, 111.2, 111.1, 110.3, 108.5, 82.9, 39.6, 37.2, 33.1, 32.0, 31.9, 30.9, 30.3, 27.0, 26.9, 26.1, 25.5, 24.8, 22.7, 21.3, 14.0, carbon attached to boron atom not observed. ${ }^{19} \mathrm{~F}\left\{{ }^{1} \mathrm{H}\right\}$ NMR (470 MHz, Chloroform- $d$ ) $\delta-80.8--80.9(\mathrm{~m}),-114.4--114.5$ 
(m), -121.85- -122.17 (m), -122.76--123.05 (m), -123.2--123.4 (m), -126.1- -126.2 (m). ${ }^{11} \mathrm{~B}$ NMR (96 MHz, Chloroform- $d$ ) $\delta$ 35.9. HRMS (ESI) $m / z:[\mathrm{M}+\mathrm{Na}]^{+}$calcd. for $\mathrm{C}_{27} \mathrm{H}_{42} \mathrm{O}_{2} \mathrm{BF}_{13} \mathrm{Na}^{+}$679.2967; found 679.2969. FTIR (neat): $v\left(\mathrm{~cm}^{-1}\right)$ 2959, 2929, 2857, 1469, 1370, 1308, 1237, 1196, 1143, 1073, 1022, 967, 854, 708, 695, 651.

4,4,5,5-Tetramethyl-2-(10,10,11,11,12,12,13,13,14,14,15,15,15-tridecafluoro-2,4,7,7tetramethylpentadecan-4-yl)-1,3,2-dioxaborolane (6d)

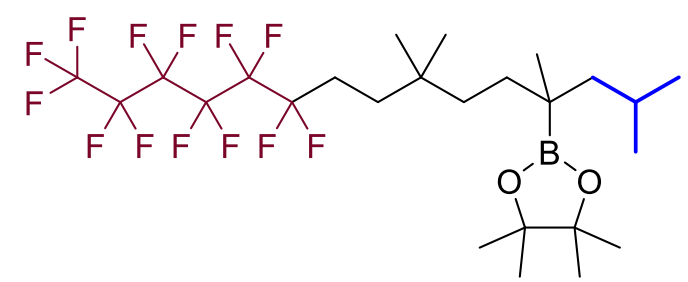

Following GP1 using 2-(5,5-dimethylhept-6-en-2-yl)-4,4,5,5-tetramethyl-1,3,2dioxaborolane (4a, $60 \mu \mathrm{L}, 50.4 \mathrm{mg}, 0.20 \mathrm{mmol})$, isopropyllithium $(0.31 \mathrm{~mL}, 0.22 \mathrm{mmol}$, 1.1 equiv, $c=0.7 \mathrm{M}$ in pentane) and 1-bromoperfluorohexane ( $64 \mu \mathrm{L}, 119.7 \mathrm{mg}, 0.3$ mmol), flash chromatography eluting with pentane/DCM (4:1) afforded 6d as light yellow sticky oil (75.8 mg, 62\% yield). $\mathrm{R}_{\mathrm{f}} 0.5$ (pentane/DCM $\left.=4: 1\right) .{ }^{1} \mathrm{H}$ NMR (300 $\mathrm{MHz}$, Chloroform- $d) \delta 2.15-1.88(\mathrm{~m}, 2 \mathrm{H}), 1.75-1.61(\mathrm{~m}, 1 \mathrm{H}), 1.52-1.42(\mathrm{~m}, 2 \mathrm{H})$, $1.42-1.33(\mathrm{~m}, 1 \mathrm{H}), 1.23(\mathrm{~s}, 12 \mathrm{H}), 1.17-1.05(\mathrm{~m}, 3 \mathrm{H}), 0.89$ (s, 3H), $0.87(\mathrm{~s}, 6 \mathrm{H}), 0.82$ $-0.79(\mathrm{~m}, 6 \mathrm{H}) .{ }^{13} \mathrm{C}\left\{{ }^{1} \mathrm{H},{ }^{19} \mathrm{~F}\right\}$ NMR $(126 \mathrm{MHz}$, Chloroform- $d) \delta 118.9,117.3,111.2$, 111.1, 110.3, 108.5, 82.9, 37.3, 33.6, 32.1, 31.0, 27.0, 26.8, 26.1, 25.0, 24.9, 19.8, 16.8, 16.5 , carbon attached to boron atom not observed. ${ }^{19} \mathrm{~F}\left\{{ }^{1} \mathrm{H}\right\}$ NMR $(470 \mathrm{MHz}$, Chloroform- $d$ ) $\delta$-78.7 - -83.6 (m), -114.4 - -114.5 (m), -121.9 - -122.1 (m), -122.8 - $123.0(\mathrm{~m}),-123.2--123.4(\mathrm{~m}),-126.1--126.3(\mathrm{~m}) .{ }^{11} \mathrm{~B}$ NMR $(96 \mathrm{MHz}$, Chloroformd) $\delta$ 35.2. HRMS (EI) m/z: $\left[\mathrm{M}-\mathrm{CH}_{3}\right]^{+}$calcd. for $\mathrm{C}_{24} \mathrm{H}_{36} \mathrm{O}_{2} \mathrm{BF}_{13}{ }^{+}$614.2600; found 614.2602. FTIR (neat): $v\left(\mathrm{~cm}^{-1}\right)$ 2961, 2936, 1471, 1370, 1306, 1237, 1196, 1142, 1119, 1072, 968, 861, 845, 811, 708, 695, 651 .

4,4,5,5-Tetramethyl-2-(9,9,10,10,11,11,12,12,13,13,14,14,14-tridecafluoro-2,2,3,6,6pentamethyltetradecan-3-yl)-1,3,2-dioxaborolane (6e)

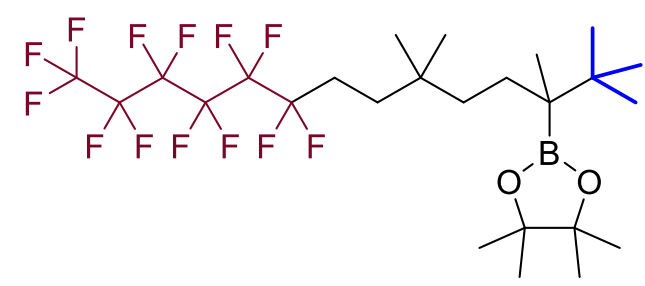

Following GP1 using 2-(5,5-dimethylhept-6-en-2-yl)-4,4,5,5-tetramethyl-1,3,2dioxaborolane (4a, $60 \mu \mathrm{L}, 50.4 \mathrm{mg}, 0.20 \mathrm{mmol})$, tert-butyllithium $(0.13 \mathrm{~mL}, 0.22 \mathrm{mmol}$, 1.1 equiv, $c=1.63 \mathrm{M}$ in pentane) and 1-bromoperfluorohexane $(64 \mu \mathrm{L}, 119.7 \mathrm{mg}, 0.3$ mmol), flash chromatography eluting with pentane/DCM (5:1) afforded 6e as colorless sticky oil $\left(56.5 \mathrm{mg}, 45 \%\right.$ yield). $\mathrm{R}_{\mathrm{f}} 0.6$ (pentane/DCM = 5:1). ${ }^{1} \mathrm{H}$ NMR (300 MHz, Chloroform- $d$ ) $\delta 2.25-1.83(\mathrm{~m}, 2 \mathrm{H}), 1.65-1.56(\mathrm{~m}, 1 \mathrm{H}), 1.51-1.42(\mathrm{~m}, 2 \mathrm{H}), 1.23(\mathrm{~s}$, $12 \mathrm{H}), 1.18-1.08(\mathrm{~m}, 1 \mathrm{H}), 1.04-0.94(\mathrm{~m}, 2 \mathrm{H}), 0.90(\mathrm{~s}, 9 \mathrm{H}), 0.88(\mathrm{~s}, 6 \mathrm{H}), 0.85(\mathrm{~s}, 3 \mathrm{H})$. 
${ }^{13} \mathrm{C}\left\{{ }^{1} \mathrm{H},{ }^{19} \mathrm{~F}\right\}$ NMR (126 MHz, Chloroform- $\left.d\right) \delta$ 119.0, 117.2, 111.2, 111.1, 110.3, 108.5, 82.9, 38.2, 34.8, 32.3, 31.1, 27.2, 27.2, 26.9, 26.2, 25.1, 25.0, 16.8, carbon attached to boron atom not observed. ${ }^{19} \mathrm{~F}\left\{{ }^{1} \mathrm{H}\right\}$ NMR $(282 \mathrm{MHz}$, Chloroform- $d$ ) $\delta-80.84(\mathrm{tt}, J=$ 10.0, $2.7 \mathrm{~Hz}),-113.95--114.55(\mathrm{~m}),-121.7--122.1(\mathrm{~m}),-122.7--123.0(\mathrm{~m}),-123.1$ $--123.4(\mathrm{~m}),-126.0--126.2(\mathrm{~m}) .{ }^{11} \mathrm{~B}$ NMR (96 MHz, Chloroform-d) $\delta$ 35.2. HRMS (EI) $m / z$ : $\left[\mathrm{M}-\mathrm{CH}_{3}\right]^{+}$calcd. for $\mathrm{C}_{24} \mathrm{H}_{35} \mathrm{O}_{2} \mathrm{BF}_{13}{ }^{+}$613.2521; found 613.2526. FTIR (neat): $v\left(\mathrm{~cm}^{-1}\right)$ 2962, 1473, 1370, 1302, 1238, 1205, 1143, 1071, 967, 855, 651 .

4,4,5,5-Tetramethyl-2-(8,8,9,9,10,10,11,11,12,12,13,13,13-tridecafluoro-5,5dimethyl-2-phenyltridecan-2-yl)-1,3,2-dioxaborolane (6f)

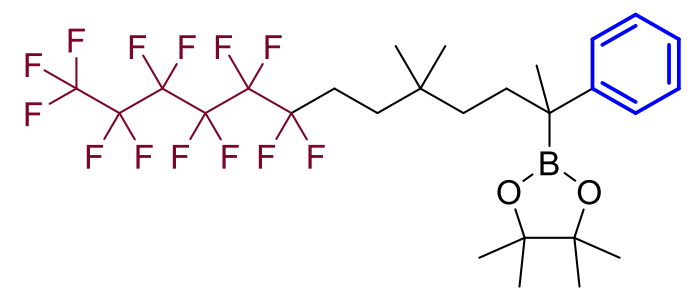

Following GP1 using 2-(5,5-dimethylhept-6-en-2-yl)-4,4,5,5-tetramethyl-1,3,2dioxaborolane (4a, $60 \mu \mathrm{L}, 50.4 \mathrm{mg}, 0.20 \mathrm{mmol})$, phenyllithium $(0.12 \mathrm{~mL}, 0.22 \mathrm{mmol}$, 1.1 equiv, $c=1.9 \mathrm{M}$ in dibutylether) and 1-bromoperfluorohexane $(64 \mu \mathrm{L}, 119.7 \mathrm{mg}$, $0.3 \mathrm{mmol})$, flash chromatography eluting with pentane/DCM (4:1) afforded 6f as colorless sticky oil $\left(87.9 \mathrm{mg}, 68 \%\right.$ yield). $\mathrm{R}_{\mathrm{f}} 0.4$ (pentane/DCM $\left.=4: 1\right) .{ }^{1} \mathrm{H}$ NMR (300 $\mathrm{MHz}$, Chloroform- $d) \delta 7.28-7.16(\mathrm{~m}, 4 \mathrm{H}), 7.10-7.01(\mathrm{~m}, 1 \mathrm{H}), 2.00-1.77(\mathrm{~m}, 2 \mathrm{H})$, $1.75-1.62(\mathrm{~m}, 1 \mathrm{H}), 1.57-1.45(\mathrm{~m}, 1 \mathrm{H}), 1.39(\mathrm{t}, J=8.5 \mathrm{~Hz}, 2 \mathrm{H}), 1.25(\mathrm{~s}, 3 \mathrm{H}), 1.13(\mathrm{~s}$, $6 \mathrm{H}), 1.12(\mathrm{~s}, 6 \mathrm{H}), 1.08-0.97(\mathrm{~m}, 2 \mathrm{H}), 0.79(\mathrm{~s}, 3 \mathrm{H}), 0.78(\mathrm{~s}, 3 \mathrm{H}) .{ }^{13} \mathrm{C}\left\{{ }^{1} \mathrm{H},{ }^{19} \mathrm{~F}\right\} \mathrm{NMR}$ (151 MHz, Chloroform-d) $\delta$ 147.3, 128.1, 126.8, 125.1, 118.9, 117.3, 111.2, 111.1, 110.3, 108.5, 83.3, 36.7, 33.5, 32.0, 30.7, 27.1, 27.0, 26.0, 24.6, 24.5, 21.6, carbon attached to boron atom not observed. ${ }^{19} \mathrm{~F}\left\{{ }^{1} \mathrm{H}\right\}$ NMR (564 MHz, Chloroform- $d$ ) $\delta-80.2$ $--81.9(\mathrm{~m}),-113.8--115.0(\mathrm{~m}),-121.9--122.1(\mathrm{~m}),-122.80--123.01(\mathrm{~m}),-123.2-$ $-123.3(\mathrm{~m}),-125.34--126.92(\mathrm{~m}) .{ }^{11} \mathrm{~B}$ NMR (192 MHz, Chloroform- $d$ ) $\delta$ 34.0. HRMS (ESI) $m / z:[\mathrm{M}+\mathrm{Na}]^{+}$calcd. for $\mathrm{C}_{27} \mathrm{H}_{34} \mathrm{O}_{2} \mathrm{BF}_{13} \mathrm{Na}^{+}$671.2341; found 671.2342. FTIR (neat): $v\left(\mathrm{~cm}^{-1}\right)$ 2979, 2876, 1472, 1350, 1313, 1237, 1204, 1192, 1143, 1120, 1067, 1030, 967, 912, 864, 847, 813, 765, 700, 653.

4,4,5,5-Tetramethyl-2-(8,8,9,9,10,10,11,11,12,12,13,13,13-tridecafluoro-2-(4fluorophenyl)-5,5-dimethyltridecan-2-yl)-1,3,2-dioxaborolane (6g)

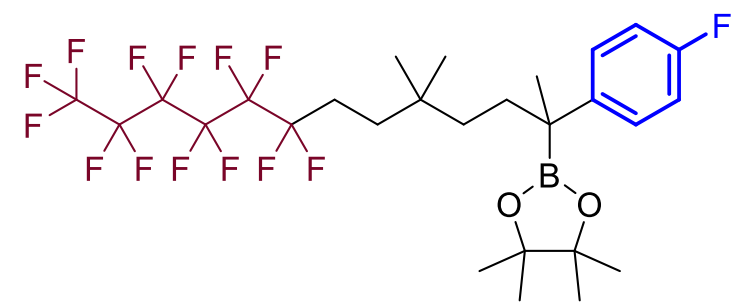

Following GP2 using 2-(5,5-dimethylhept-6-en-2-yl)-4,4,5,5-tetramethyl-1,3,2dioxaborolane (4a, $60 \mu \mathrm{L}, 50.4 \mathrm{mg}, 0.20 \mathrm{mmol}$ ), 1-fluoro-4-iodobenzene ( $25.4 \mu \mathrm{L}, 48.8$ $\mathrm{mg}, 0.22 \mathrm{mmol})$ and 1-bromoperfluorohexane $(64 \mu \mathrm{L}, 119.7 \mathrm{mg}, 0.3 \mathrm{mmol})$, flash 
chromatography eluting with pentane/DCM (4:1) afforded $\mathbf{6 g}$ as colorless sticky oil (86.6 mg, 65\% yield). $\mathrm{R}_{\mathrm{f}} 0.4$ (pentane/DCM = 4:1). ${ }^{1} \mathrm{H}$ NMR (300 MHz, Chloroformd) $\delta 7.23-7.15(\mathrm{~m}, 2 \mathrm{H}), 6.93-6.84(\mathrm{~m}, 2 \mathrm{H}), 2.01-1.73(\mathrm{~m}, 2 \mathrm{H}), 1.70-1.57(\mathrm{~m}, 1 \mathrm{H})$, $1.55-1.45(\mathrm{~m}, 1 \mathrm{H}), 1.44-1.33(\mathrm{~m}, 2 \mathrm{H}), 1.23(\mathrm{~s}, 3 \mathrm{H}), 1.13(\mathrm{~s}, 6 \mathrm{H}), 1.12(\mathrm{~s}, 6 \mathrm{H}), 1.05$ - $0.96(\mathrm{~m}, 2 \mathrm{H}), 0.78(\mathrm{~s}, 3 \mathrm{H}), 0.78(\mathrm{~s}, 3 \mathrm{H}) .{ }^{13} \mathrm{C}\left\{{ }^{1} \mathrm{H},{ }^{19} \mathrm{~F}\right\}$ NMR $(126 \mathrm{MHz}$, Chloroformd) $\delta 160.8,142.8,128.2,118.9,117.2,114.7,111.2,111.1,110.3,108.5,83.4,36.7,33.7$, $31.9,30.7,27.1,26.9,26.0,24.6,24.5,21.7$, carbon attached to boron atom not observed. ${ }^{19} \mathrm{~F}\left\{{ }^{1} \mathrm{H}\right\}$ NMR $(470 \mathrm{MHz}$, Chloroform- $d$ ) $\delta-80.8--80.9(\mathrm{~m}),-113.4--115.5$ (m), -118.9, -121.8 - -122.1 (m), -122.8 - -123.0 (m), -123.2 - -123.3 (m), -126.1 - $126.2(\mathrm{~m}) .{ }^{11} \mathrm{~B}$ NMR $\left(96 \mathrm{MHz}, \mathrm{CDCl}_{3}\right) \delta 34.7$. HRMS (EI) $\mathrm{m} / \mathrm{z}:[\mathrm{M}]^{+}$calcd. for $\mathrm{C}_{27} \mathrm{H}_{33} \mathrm{O}_{2} \mathrm{BF}_{14}{ }^{+}$666.2349, found 666.2360.

2-(2-(4-Chlorophenyl)-8,8,9,9,10,10,11,11,12,12,13,13,13-tridecafluoro-5,5dimethyltridecan-2-yl)-4,4,5,5-tetramethyl-1,3,2-dioxaborolane (6h)

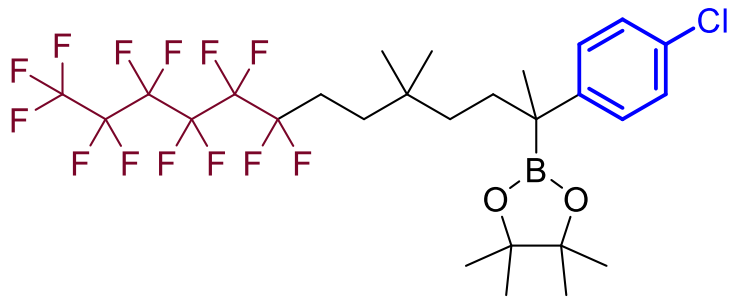

Following GP2 using 2-(5,5-dimethylhept-6-en-2-yl)-4,4,5,5-tetramethyl-1,3,2dioxaborolane (4a, $60 \mu \mathrm{L}, 50.4 \mathrm{mg}, 0.20 \mathrm{mmol}$ ), 1-chloro-4-iodobenzene (52.5 mg, $0.22 \mathrm{mmol})$ and 1-bromoperfluorohexane $(64 \mu \mathrm{L}, 119.7 \mathrm{mg}, 0.3 \mathrm{mmol})$, flash chromatography eluting with pentane/DCM (4:1) afforded $\mathbf{6 h}$ as colorless sticky oil (97.5 mg, 71\% yield). $\mathrm{R}_{\mathrm{f}} 0.5$ (pentane/DCM = 3:1). ${ }^{1} \mathrm{H}$ NMR (300 MHz, Chloroformd) $\delta 7.19-7.14(\mathrm{~m}, 4 \mathrm{H}), 2.04-1.75(\mathrm{~m}, 2 \mathrm{H}), 1.70-1.57(\mathrm{~m}, 1 \mathrm{H}), 1.55-1.46(\mathrm{~m}, 1 \mathrm{H})$, $1.45-1.31(\mathrm{~m}, 2 \mathrm{H}), 1.22(\mathrm{~s}, 3 \mathrm{H}), 1.12(\mathrm{~s}, 6 \mathrm{H}), 1.11(\mathrm{~s}, 6 \mathrm{H}), 1.05-0.96(\mathrm{~m}, 2 \mathrm{H}), 0.78$ (s, 3H), $0.78(\mathrm{~s}, 3 \mathrm{H}) .{ }^{13} \mathrm{C}\left\{{ }^{1} \mathrm{H},{ }^{19} \mathrm{~F}\right\}$ NMR (126 MHz, Chloroform-d) $\delta$ 145.7, 130.9, 128.2, 128.2, 118.9, 117.2, 111.2, 111.1, 110.3, 108.5, 83.5, 36.7, 33.5, 32.0, 30.7, 27.0, 26.9, 26.0, 24.5, 24.5, 21.5, carbon attached to boron atom not observed. ${ }^{19} \mathrm{~F}\left\{{ }^{1} \mathrm{H}\right\} \mathrm{NMR}$ (282 MHz, Chloroform- $d$ ) $\delta-80.8--80.9(\mathrm{~m}),-114.1--114.4(\mathrm{~m}),-121.7--122.1(\mathrm{~m})$, $-122.7--123.0(\mathrm{~m}),-123.0--123.4(\mathrm{~m}),-125.9--126.4(\mathrm{~m}) .{ }^{11} \mathrm{~B}$ NMR $(96 \mathrm{MHz}$, Chloroform- $d$ ) $\delta$ 34.6. HRMS (EI) $m / z$ : $\left[\mathrm{M}-\mathrm{CH}_{3}\right]^{+}$calcd. for $\mathrm{C}_{26} \mathrm{H}_{30} \mathrm{O}_{2} \mathrm{BClF}_{13}{ }^{+}$ 667.1819; found 667.1830. FTIR (neat): $v\left(\mathrm{~cm}^{-1}\right)$ 2962, 1492, 1349, 1317, 1237, 1202, 1142, 1115, 1014, 967, 910, 847, 827, 727, 706, 651.

2-(2-(4-Bromophenyl)-8,8,9,9,10,10,11,11,12,12,13,13,13-tridecafluoro-5,5dimethyltridecan-2-yl)-4,4,5,5-tetramethyl-1,3,2-dioxaborolane (6i)<smiles>CC(C)(CCC(C)(B1OC(C)(C)C(C)(C)O1)c1ccc(Br)cc1)CCC(F)(F)C(F)(F)C(F)(F)C(F)(F)C(F)(F)C(F)(F)C(F)(F)F</smiles> 
Following GP2 using 2-(5,5-dimethylhept-6-en-2-yl)-4,4,5,5-tetramethyl-1,3,2dioxaborolane (4a, $60 \mu \mathrm{L}, 50.4 \mathrm{mg}, 0.20 \mathrm{mmol}), 1,4$-dibromobenzene (51.9 mg, 0.22 mmol) and 1-bromoperfluorohexane (64 $\mu \mathrm{L}, 119.7 \mathrm{mg}, 0.3 \mathrm{mmol})$, flash chromatography eluting with pentane/DCM (4:1) afforded $\mathbf{6 i}$ as colorless sticky oil (94.9 mg, 65\% yield). $\mathrm{R}_{\mathrm{f}} 0.4$ (pentane/DCM = 4:1). ${ }^{1} \mathrm{H}$ NMR $(300 \mathrm{MHz}$, Chloroformd) $\delta 7.35-7.27(\mathrm{~m}, 2 \mathrm{H}), 7.15-7.08(\mathrm{~m}, 2 \mathrm{H}), 2.03-1.74(\mathrm{~m}, 2 \mathrm{H}), 1.70-1.56(\mathrm{~m}, 1 \mathrm{H})$, $1.55-1.45(\mathrm{~m}, 1 \mathrm{H}), 1.45-1.30(\mathrm{~m}, 2 \mathrm{H}), 1.22(\mathrm{~s}, 3 \mathrm{H}), 1.12(\mathrm{~s}, 6 \mathrm{H}), 1.11(\mathrm{~s}, 6 \mathrm{H}), 1.05$ - $0.95(\mathrm{~m}, 2 \mathrm{H}), 0.78(\mathrm{~s}, 3 \mathrm{H}), 0.78(\mathrm{~s}, 3 \mathrm{H}) .{ }^{13} \mathrm{C}\left\{{ }^{1} \mathrm{H},{ }^{19} \mathrm{~F}\right\}$ NMR $(126 \mathrm{MHz}$, Chloroformd) $\delta 146.3,131.1,128.7,119.0,118.9,117.2,111.2,111.1,110.3,108.5,83.5,36.7,33.4$, $32.0,30.7,27.0,26.9,26.0,24.6,24.5,21.4$, carbon attached to boron atom not observed. ${ }^{19} \mathrm{~F}\left\{{ }^{1} \mathrm{H}\right\}$ NMR $(470 \mathrm{MHz}$, Chloroform- $d) \delta-80.8--80.9(\mathrm{~m}),-114.2--114.4$ (m), -121.8--122.1 (m), -122.7--123.0 (m), -123.1--123.4 (m), -126.0--126.3 (m). ${ }^{11} \mathrm{~B}$ NMR (96 MHz, Chloroform- $d$ ) $\delta$ 34.4. HRMS (ESI) $\mathrm{m} / \mathrm{z}:[\mathrm{M}+\mathrm{Na}]^{+}$calcd. for $\mathrm{C}_{27} \mathrm{H}_{33} \mathrm{O}_{2} \mathrm{BBrF}_{13} \mathrm{Na}^{+} 749.1442$; found 749.1450. FTIR (neat): $v\left(\mathrm{~cm}^{-1}\right) 2962,1472$, 1349, 1317, 1237, 1203, 1142, 1079, 1009, 967, 909, 847, 733, 651.

4,4,5,5-Tetramethyl-2-(8,8,9,9,10,10,11,11,12,12,13,13,13-tridecafluoro-2-(4iodophenyl)-5,5-dimethyltridecan-2-yl)-1,3,2-dioxaborolane (6j)

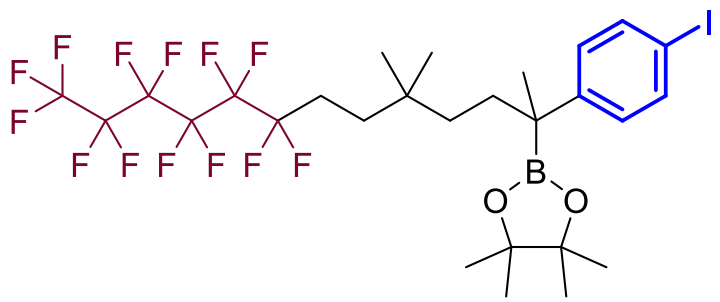

Following GP2 using 2-(5,5-dimethylhept-6-en-2-yl)-4,4,5,5-tetramethyl-1,3,2dioxaborolane (4a, $60 \mu \mathrm{L}, 50.4 \mathrm{mg}, 0.20 \mathrm{mmol})$, 1,4-diiodobenzene (72.6 mg, 0.22 mmol) and 1-bromoperfluorohexane $(64 \mu \mathrm{L}, 119.7 \mathrm{mg}, 0.3 \mathrm{mmol})$, flash chromatography eluting with pentane/DCM (4:1) afforded $\mathbf{6 j}$ as colorless sticky oil (98.4 mg, 64\% yield). $\mathrm{R}_{\mathrm{f}} 0.6$ (pentane/DCM = 3:1). ${ }^{1} \mathrm{H}$ NMR $(300 \mathrm{MHz}$, Chloroformd) $\delta 7.64-7.55(\mathrm{~m}, 2 \mathrm{H}), 7.11-7.04(\mathrm{~m}, 2 \mathrm{H}), 2.13-1.84(\mathrm{~m}, 2 \mathrm{H}), 1.78-1.64(\mathrm{~m}, 1 \mathrm{H})$, $1.63-1.52(\mathrm{~m}, 1 \mathrm{H}), 1.52-1.41(\mathrm{~m}, 2 \mathrm{H}), 1.29(\mathrm{~s}, 3 \mathrm{H}), 1.20(\mathrm{~s}, 6 \mathrm{H}), 1.19(\mathrm{~s}, 6 \mathrm{H}), 0.86$ (s, 3H), $0.86(\mathrm{~s}, 3 \mathrm{H}) .{ }^{13} \mathrm{C}\left\{{ }^{1} \mathrm{H},{ }^{19} \mathrm{~F}\right\}$ NMR $(126 \mathrm{MHz}$, Chloroform- $d$ ) $\delta$ 147.1, 137.1, 129.1, 118.9, 117.2, 111.2, 111.1, 110.3, 108.5, 90.4, 83.5, 36.7, 33.4, 32.0, 30.7, 27.0, 26.9, 26.0, 24.6, 24.5, 21.4, carbon attached to boron atom not observed. ${ }^{19} \mathrm{~F}\left\{{ }^{1} \mathrm{H}\right\} \mathrm{NMR}$ $(470 \mathrm{MHz}$, Chloroform- $d$ ) $\delta$-80.82 - -80.91 (m), -114.20 - -114.44 (m), -121.83 - $122.13(\mathrm{~m}),-122.72--122.98(\mathrm{~m}),-123.14--123.29(\mathrm{~m}),-126.07--126.23(\mathrm{~m}) .{ }^{11} \mathrm{~B}$ $\mathrm{NMR}\left(96 \mathrm{MHz}, \mathrm{CDCl}_{3}\right) \delta$ 33.9. HRMS (ESI) $\mathrm{m} / \mathrm{z}:[\mathrm{M}+\mathrm{Na}]^{+}$calcd. for $\mathrm{C}_{27} \mathrm{H}_{33} \mathrm{O}_{2} \mathrm{BF}_{13} \mathrm{INa}^{+}$797.1308; found 797.1311. FTIR (neat): $v\left(\mathrm{~cm}^{-1}\right)$ 2963, 2873, 1735 , 1700, 1685, 1652, 1558, 1473, 1317, 1236, 1196, 1142, 1071, 1004, 847, 818, 735, 692, 651.

4,4,5,5-Tetramethyl-2-(8,8,9,9,10,10,11,11,12,12,13,13,13-tridecafluoro-5,5dimethyl-2-(4-(trifluoromethyl)phenyl)tridecan-2-yl)-1,3,2-dioxaborolane (6k) 


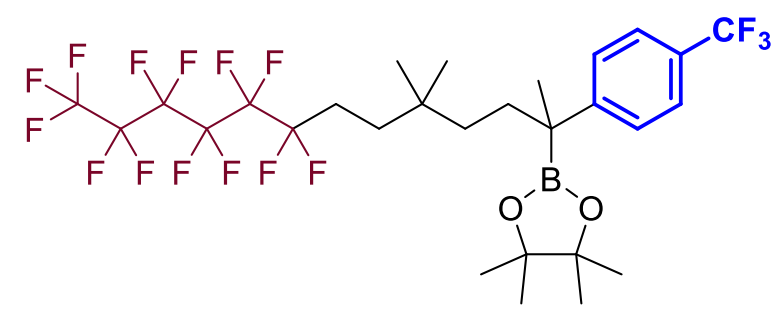

Following GP2 using 2-(5,5-dimethylhept-6-en-2-yl)-4,4,5,5-tetramethyl-1,3,2dioxaborolane (4a, $60 \mu \mathrm{L}, 50.4 \mathrm{mg}, 0.20 \mathrm{mmol}$ ), 4-iodotrifluorotoluene (60.9 mg, 0.22 mmol) and 1-bromoperfluorohexane (64 $\mu \mathrm{L}, 119.7 \mathrm{mg}, 0.3 \mathrm{mmol})$, flash chromatography eluting with pentane/DCM (4:1) afforded $\mathbf{6 k}$ as colorless sticky oil (92.4 mg, 65\% yield). $\mathrm{R}_{\mathrm{f}} 0.4$ (pentane/DCM = 4:1). ${ }^{1} \mathrm{H}$ NMR (300 MHz, Chloroformd) $\delta 7.45(\mathrm{~d}, J=8.3 \mathrm{~Hz}, 2 \mathrm{H}), 7.39-7.30(\mathrm{~m}, 2 \mathrm{H}), 2.02-1.72(\mathrm{~m}, 2 \mathrm{H}), 1.76-1.60(\mathrm{~m}$, $1 \mathrm{H}), 1.62-1.45(\mathrm{~m}, 1 \mathrm{H}), 1.44-1.32(\mathrm{~m}, 2 \mathrm{H}), 1.27(\mathrm{~s}, 3 \mathrm{H}), 1.13(\mathrm{~s}, 6 \mathrm{H}), 1.12(\mathrm{~s}, 6 \mathrm{H})$, $1.08-0.94(\mathrm{~m}, 2 \mathrm{H}), 0.79(\mathrm{~s}, 3 \mathrm{H}), 0.78(\mathrm{~s}, 3 \mathrm{H}) .{ }^{13} \mathrm{C}\left\{{ }^{1} \mathrm{H},{ }^{19} \mathrm{~F}\right\}$ NMR (126 MHz, Chloroform- $d$ ) $\delta 151.5,127.5,127.1,125.0,124.5,118.9$, 117.3, 111.2, 111.1, 110.3, 108.5, 83.6, 36.6, 33.4, 32.0, 30.6, 27.0, 26.9, 26.0, 24.6, 24.5, 21.4, carbon attached to boron atom not observed. ${ }^{19} \mathrm{~F}\left\{{ }^{1} \mathrm{H}\right\}$ NMR $(470 \mathrm{MHz}$, Chloroform- $d$ ) $\delta-80.9(\mathrm{~m}),-$ $114.3--114.5(\mathrm{~m}),-121.8--122.1(\mathrm{~m}),-122.8--123.1(\mathrm{~m}),-123.1--123.4(\mathrm{~m})$, $126.1--126.3(\mathrm{~m}) .{ }^{11} \mathrm{~B} \mathrm{NMR}\left(96 \mathrm{MHz}, \mathrm{CDCl}_{3}\right) \delta 32.6$. HRMS (ESI) $m / z:[\mathrm{M}+\mathrm{Na}]^{+}$ calcd. for $\mathrm{C}_{28} \mathrm{H}_{33} \mathrm{O}_{2} \mathrm{BF}_{16} \mathrm{Na}^{+}$739.2215; found 739.2217. FTIR (neat): v ( $\left.\mathrm{cm}^{-1}\right)$ 2978, 2932, 1618, 1473, 1382, 1350, 1328, 1240, 1208, 1167, 1144, 1127, 1073, 1017, 910, $846,736,707$.

4,4,5,5-Tetramethyl-2-(8,8,9,9,10,10,11,11,12,12,13,13,13-tridecafluoro-5,5dimethyl-2-(4-(trifluoromethoxy)phenyl)tridecan-2-yl)-1,3,2-dioxaborolane (6l)

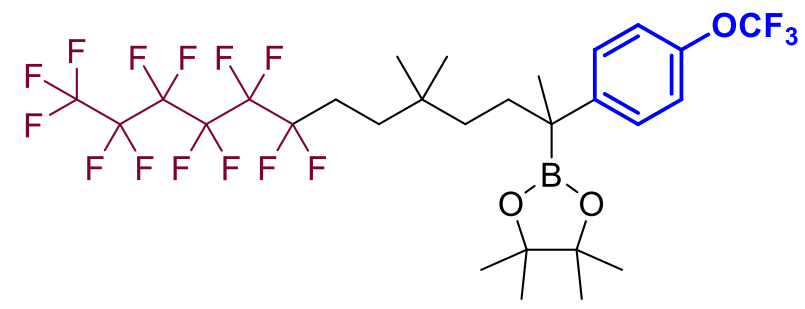

Following GP2 using 2-(5,5-dimethylhept-6-en-2-yl)-4,4,5,5-tetramethyl-1,3,2dioxaborolane (4a, $60 \mu \mathrm{L}, 50.4 \mathrm{mg}, 0.20 \mathrm{mmol}$ ), 1-iodo-4-(trifluoromethoxy)benzene (63.4 mg, $0.22 \mathrm{mmol}$ ) and 1-bromoperfluorohexane (64 $\mu \mathrm{L}, 119.7 \mathrm{mg}, 0.3 \mathrm{mmol})$, flash chromatography eluting with pentane/DCM (4:1) afforded $\mathbf{6 1}$ as colorless sticky oil (89.7 mg, 61\% yield). $\mathrm{R}_{\mathrm{f}} 0.5$ (pentane/DCM = 4:1). ${ }^{1} \mathrm{H}$ NMR (300 MHz, Chloroformd) $\delta 7.29-7.21(\mathrm{~m}, 2 \mathrm{H}), 7.09-6.99(\mathrm{~m}, 2 \mathrm{H}), 2.04-1.74(\mathrm{~m}, 2 \mathrm{H}), 1.72-1.58(\mathrm{~m}, 1 \mathrm{H})$, $1.56-1.43(\mathrm{~m}, 1 \mathrm{H}), 1.38(\mathrm{t}, J=8.6 \mathrm{~Hz}, 2 \mathrm{H}), 1.24(\mathrm{~s}, 3 \mathrm{H}), 1.13(\mathrm{~s}, 6 \mathrm{H}), 1.12(\mathrm{~s}, 6 \mathrm{H})$, $0.79(\mathrm{~s}, 3 \mathrm{H}), 0.78(\mathrm{~s}, 3 \mathrm{H}) .{ }^{13} \mathrm{C}\left\{{ }^{1} \mathrm{H},{ }^{19} \mathrm{~F}\right\} \mathrm{NMR}(126 \mathrm{MHz}$, Chloroform- $d) \delta 146.9,145.9$, 128.0, 120.4, 118.9, 117.2, 111.2, 111.1, 110.3, 108.5, 83.5, 36.7, 33.6, 31.9, 30.6, 27.0, $26.9,26.0,24.5,24.5,21.7$, carbon attached to boron atom not observed. ${ }^{19} \mathrm{~F}\left\{{ }^{1} \mathrm{H}\right\} \mathrm{NMR}$ (470 MHz, Chloroform- $d$ ) $\delta-80.9(\mathrm{~m}),-114.3--114.5(\mathrm{~m}),-121.8--122.1(\mathrm{~m}),-122.7$ $-123.0(\mathrm{~m}),-123.2--123.4(\mathrm{~m}),-126.1--126.3(\mathrm{~m}) .{ }^{11} \mathrm{~B} \mathrm{NMR}\left(96 \mathrm{MHz}, \mathrm{CDCl}_{3}\right) \delta$ 32.4. HRMS (ESI) $m / z:[\mathrm{M}+\mathrm{Na}]^{+}$calcd. for $\mathrm{C}_{28} \mathrm{H}_{33} \mathrm{O}_{3} \mathrm{BF}_{16} \mathrm{Na}^{+}$755.2160; found: 
755.2169. FTIR (neat): $v\left(\mathrm{~cm}^{-1}\right) 2974,2876,1508,1472,1382,1351,1317,1261,1238$, 1210, 1166, 1144, 1121, 1070, 967, 909, 850, 811, 736, 708, 694, 652.

4,4,5,5-Tetramethyl-2-(8,8,9,9,10,10,11,11,12,12,13,13,13-tridecafluoro-5,5dimethyl-2-(p-tolyl)tridecan-2-yl)-1,3,2-dioxaborolane (6m)

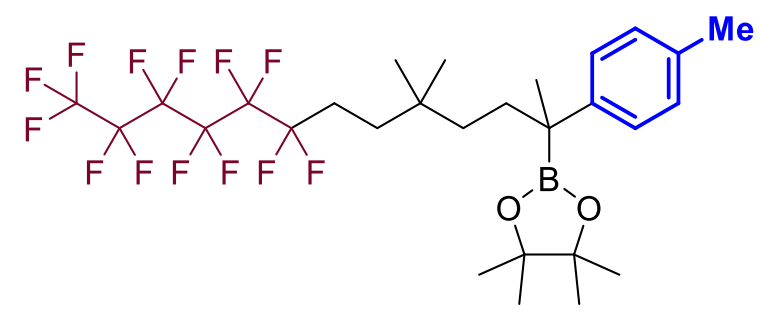

Following GP2 using 2-(5,5-dimethylhept-6-en-2-yl)-4,4,5,5-tetramethyl-1,3,2dioxaborolane (4a, $60 \mu \mathrm{L}, 50.4 \mathrm{mg}, 0.20 \mathrm{mmol})$, 4-iodotoluene (48.0 mg, $0.22 \mathrm{mmol})$ and 1-bromoperfluorohexane ( $64 \mu \mathrm{L}, 119.7 \mathrm{mg}, 0.3 \mathrm{mmol})$, flash chromatography eluting with pentane/DCM (4:1) afforded $\mathbf{6 m}$ as colorless sticky oil $(81.2 \mathrm{mg}, 61 \%$ yield). $\mathrm{R}_{\mathrm{f}} 0.5$ (pentane/DCM = 4:1). ${ }^{1} \mathrm{H}$ NMR (300 MHz, Chloroform- $d$ ) $\delta 7.15-7.09$ $(\mathrm{m}, 2 \mathrm{H}), 7.04-6.98(\mathrm{~m}, 2 \mathrm{H}), 2.22(\mathrm{~s}, 3 \mathrm{H}), 2.04-1.72(\mathrm{~m}, 2 \mathrm{H}), 1.73-1.59(\mathrm{~m}, 1 \mathrm{H})$, $1.53-1.42(\mathrm{~m}, 1 \mathrm{H}), 1.38(\mathrm{t}, J=8.6 \mathrm{~Hz}, 3 \mathrm{H}), 1.22(\mathrm{~s}, 3 \mathrm{H}), 1.13(\mathrm{~s}, 6 \mathrm{H}), 1.11(\mathrm{~s}, 6 \mathrm{H})$, $1.08-0.99(\mathrm{~m}, 2 \mathrm{H}), 0.79(\mathrm{~s}, 3 \mathrm{H}), 0.78(\mathrm{~s}, 3 \mathrm{H}) .{ }^{13} \mathrm{C}\left\{{ }^{1} \mathrm{H},{ }^{19} \mathrm{~F}\right\}$ NMR $(126 \mathrm{MHz}$, Chloroform- $d$ ) $\delta 144.2,134.4,128.8,126.6,119.0,117.2,111.2,111.1,110.3,108.5$, 83.3, 36.7, 33.6, 32.0, 30.7, 27.1, 27.0, 26.0, 24.6, 24.5, 21.7, 20.8, carbon attached to boron atom not observed. ${ }^{19} \mathrm{~F}\left\{{ }^{1} \mathrm{H}\right\}$ NMR $(470 \mathrm{MHz}$, Chloroform- $d) \delta-80.9(\mathrm{~m}),-114.3$ (m), -121.8--122.1 (m), -122.7--123.1 (m), -123.1--123.4 (m), -126.0--126.3 (m). ${ }^{11} \mathrm{~B}$ NMR $\left(96 \mathrm{MHz}, \mathrm{CDCl}_{3}\right) \delta$ 33.6. HRMS (ESI) $\mathrm{m} / \mathrm{z}:[\mathrm{M}+\mathrm{Na}]^{+}$calcd. for $\mathrm{C}_{28} \mathrm{H}_{36} \mathrm{O}_{2} \mathrm{BF}_{13} \mathrm{Na}^{+}$685.2498; found 685.2497. FTIR (neat): $v\left(\mathrm{~cm}^{-1}\right)$ 2979, 2874, 1513, 1470, 1349, 1310, 1349, 1310, 1238, 1205, 1193, 1144, 1119, 1068,1020, 967, 909, 865, $848,816,734,707,694,651$.

4,4,5,5-Tetramethyl-2-(8,8,9,9,10,10,11,11,12,12,13,13,13-tridecafluoro-2-(4methoxyphenyl)-5,5-dimethyltridecan-2-yl)-1,3,2-dioxaborolane (6n)

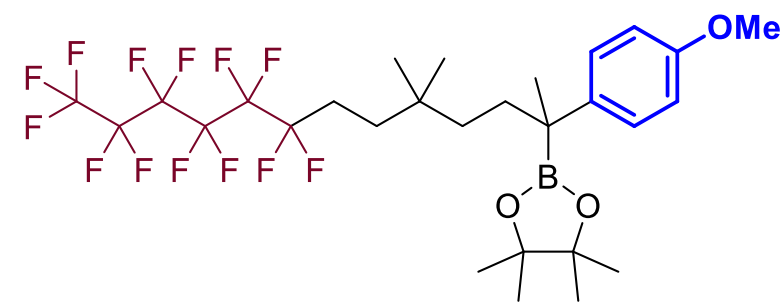

Following GP2 using 2-(5,5-dimethylhept-6-en-2-yl)-4,4,5,5-tetramethyl-1,3,2dioxaborolane (4a, $60 \mu \mathrm{L}, 50.4 \mathrm{mg}, 0.20 \mathrm{mmol})$, 4-iodoanisole $(51.5 \mathrm{mg}, 0.22 \mathrm{mmol})$ and 1-bromoperfluorohexane (64 $\mu \mathrm{L}, 119.7 \mathrm{mg}, 0.3 \mathrm{mmol})$, flash chromatography eluting with pentane/DCM (1:1) afforded 6n as colorless sticky oil (68.4 mg, 50\% yield). $\mathrm{R}_{\mathrm{f}} 0.6$ (pentane/DCM = 1:1). ${ }^{1} \mathrm{H}$ NMR $(300 \mathrm{MHz}$, Chloroform- $d$ ) $\delta 7.17-7.11$ $(\mathrm{m}, 2 \mathrm{H}), 6.79-6.71(\mathrm{~m}, 2 \mathrm{H}), 3.70(\mathrm{~s}, 3 \mathrm{H}), 2.05-1.74(\mathrm{~m}, 2 \mathrm{H}), 1.71-1.57(\mathrm{~m}, 1 \mathrm{H})$, $1.54-1.32(\mathrm{~m}, 3 \mathrm{H}), 1.22(\mathrm{~s}, 3 \mathrm{H}), 1.12(\mathrm{~s}, 6 \mathrm{H}), 1.11(\mathrm{~s}, 6 \mathrm{H}), 1.07-0.98(\mathrm{~m}, 2 \mathrm{H}), 0.78$ (s, 3H), 0.78 (s, 3H). ${ }^{13} \mathrm{C}\left\{{ }^{1} \mathrm{H},{ }^{19} \mathrm{~F}\right\}$ NMR $\left(126 \mathrm{MHz}, \mathrm{CDCl}_{3}\right) \delta 157.2,139.2,127.7$, 
118.9, 117.2, 113.5, 111.2, 111.1, 110.3, 108.5, 83.3, 55.1, 36.8, 33.7, 32.0, 30.7, 27.1, 27.0, 26.0, 24.6, 24.5, 21.8, carbon attached to boron atom not observed. ${ }^{19} \mathrm{~F}\left\{{ }^{1} \mathrm{H}\right\} \mathrm{NMR}$ $(470 \mathrm{MHz}$, Chloroform- $d$ ) $\delta-80.8--80.9(\mathrm{~m}),-114.3(\mathrm{~m}),-121.8--122.1(\mathrm{~m}),-122.8$ $-123.0(\mathrm{~m}),-123.1--123.4(\mathrm{~m}),-126.0--126.3(\mathrm{~m}) .{ }^{11} \mathrm{~B} \mathrm{NMR}\left(96 \mathrm{MHz}, \mathrm{CDCl}_{3}\right) \delta$ 33.9. HRMS (ESI) $m / z$ : $[\mathrm{M}+\mathrm{Na}]^{+}$calcd. for $\mathrm{C}_{28} \mathrm{H}_{36} \mathrm{O}_{3} \mathrm{BF}_{13} \mathrm{Na}^{+}$701.2449; found 701.2447. FTIR (neat): $v\left(\mathrm{~cm}^{-1}\right)$ 2959, 1718, 1604, 1512, 1367, 1236, 1206, 1144, 1120 , 1039, 828, 734, 653 .

4,4,5,5-Tetramethyl-2-(8,8,9,9,10,10,11,11,12,12,13,13,13-tridecafluoro-2-(3fluorophenyl)-5,5-dimethyltridecan-2-yl)-1,3,2-dioxaborolane (6o)

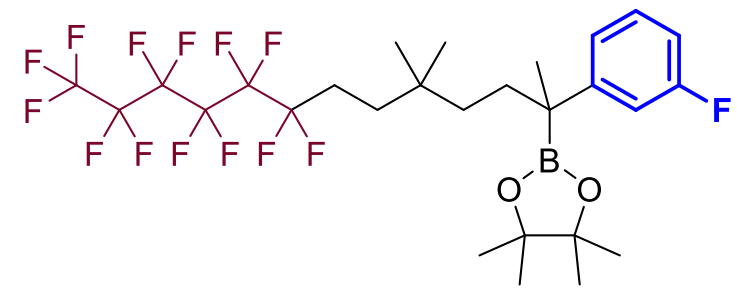

Following GP2 using 2-(5,5-dimethylhept-6-en-2-yl)-4,4,5,5-tetramethyl-1,3,2dioxaborolane (4a, $60 \mu \mathrm{L}, 50.4 \mathrm{mg}, 0.20 \mathrm{mmol}$ ), 3-fluoroiodobenzene (48.8 mg, 0.22 $\mathrm{mmol}$ ) and 1-bromoperfluorohexane (64 $\mu \mathrm{L}, 119.7 \mathrm{mg}, 0.3 \mathrm{mmol})$, flash chromatography eluting with pentane/DCM (4:1) afforded 60 as colorless sticky oil (82.4 mg, 62\% yield). $\mathrm{R}_{\mathrm{f}} 0.4$ (pentane/DCM = 4:1). ${ }^{1} \mathrm{H}$ NMR (300 MHz, Chloroformd) $\delta 7.20-7.10(\mathrm{~m}, 1 \mathrm{H}), 7.04-6.91(\mathrm{~m}, 2 \mathrm{H}), 6.80-6.70(\mathrm{~m}, 1 \mathrm{H}), 2.03-1.74(\mathrm{~m}, 2 \mathrm{H})$, $1.72-1.59(\mathrm{~m}, 1 \mathrm{H}), 1.57-1.44(\mathrm{~m}, 1 \mathrm{H}), 1.38(\mathrm{t}, J=8.6 \mathrm{~Hz}, 2 \mathrm{H}), 1.23(\mathrm{~s}, 3 \mathrm{H}), 1.13(\mathrm{~s}$, $6 \mathrm{H}), 1.12(\mathrm{~s}, 6 \mathrm{H}), 1.06-0.97(\mathrm{~m}, 2 \mathrm{H}), 0.79(\mathrm{~s}, 3 \mathrm{H}), 0.78(\mathrm{~s}, 3 \mathrm{H}),{ }^{13} \mathrm{C}\left\{{ }^{1} \mathrm{H},{ }^{19} \mathrm{~F}\right\} \mathrm{NMR}$ $\left(126 \mathrm{MHz}, \mathrm{CDCl}_{3}\right) \delta 163.0,163.0,150.2,129.3,122.5,118.9,117.2,113.8,111.9,111.2$, 111.10, 110.3, 108.5, 83.5, 36.7, 33.4, 31.9, 30.7, 27.0, 26.9, 26.0, 24.5, 24.5, 21.4, carbon attached to boron atom not observed. ${ }^{19} \mathrm{~F}\left\{{ }^{1} \mathrm{H}\right\}$ NMR $(470 \mathrm{MHz}$, Chloroform- $d)$ $\delta-80.8--80.9(\mathrm{~m}),-113.8(\mathrm{~s}),-114.3--114.4(\mathrm{~m}),-121.8--122.1(\mathrm{~m}),-122.8--123.0$ (m), -123.1 - -123.4 (m), -126.0 - -126.3 (m). ${ }^{11} \mathrm{~B}$ NMR $\left(96 \mathrm{MHz}, \mathrm{CDCl}_{3}\right) \delta 32.9$. HRMS (ESI) $m / z$ : $[\mathrm{M}+\mathrm{Na}]^{+}$calcd. for $\mathrm{C}_{27} \mathrm{H}_{33} \mathrm{O}_{2} \mathrm{BF}_{14} \mathrm{Na}^{+}$689.2247; found 689.2249. FTIR (neat): $v\left(\mathrm{~cm}^{-1}\right)$ 2980, 1613, 1585, 1471, 1351, 1317, 1236, 1194, 1166, 1142, 1120, 1071, 1016, 967, 909, 856, 812, 783, 735, 707, 694, 680, 650 .

2-(2-(3-Chlorophenyl)-8,8,9,9,10,10,11,11,12,12,13,13,13-tridecafluoro-5,5dimethyltridecan-2-yl)-4,4,5,5-tetramethyl-1,3,2-dioxaborolane (6p)<smiles>CC(C)(CCC(C)(B1OC(C)(C)C(C)(C)O1)c1cccc(Cl)c1)CCC(F)(F)C(F)(F)C(F)(F)C(F)(F)C(F)(F)C(F)(F)F</smiles>

Following GP2 using 2-(5,5-dimethylhept-6-en-2-yl)-4,4,5,5-tetramethyl-1,3,2dioxaborolane (4a, $60 \mu \mathrm{L}, 50.4 \mathrm{mg}, 0.20 \mathrm{mmol}$ ), 3-chloroiodobenzene (52.5 mg, 0.22 $\mathrm{mmol})$ and 1-bromoperfluorohexane $(64 \mu \mathrm{L}, 119.7 \mathrm{mg}, 0.3 \mathrm{mmol})$, flash 
chromatography eluting with pentane/DCM (4:1) afforded 6p as colorless sticky oil (97.7 mg, 72\% yield). $\mathrm{R}_{\mathrm{f}} 0.5$ (pentane/DCM = 3:1). ${ }^{1} \mathrm{H}$ NMR (300 MHz, Chloroformd) $\delta 7.24-7.20(\mathrm{~m}, 1 \mathrm{H}), 7.15-7.09(\mathrm{~m}, 2 \mathrm{H}), 7.08-7.00(\mathrm{~m}, 1 \mathrm{H}), 2.08-1.74(\mathrm{~m}, 2 \mathrm{H})$, $1.72-1.59(\mathrm{~m}, 1 \mathrm{H}), 1.56-1.46(\mathrm{~m}, 1 \mathrm{H}), 1.45-1.32(\mathrm{~m}, 2 \mathrm{H}), 1.23(\mathrm{~s}, 3 \mathrm{H}), 1.13(\mathrm{~s}$, 6H), $1.12(\mathrm{~s}, 6 \mathrm{H}), 1.07-0.95(\mathrm{~m}, 2 \mathrm{H}), 0.79(\mathrm{~s}, 3 \mathrm{H}), 0.79(\mathrm{~s}, 3 \mathrm{H}) \cdot{ }^{13} \mathrm{C}\left\{{ }^{1} \mathrm{H},{ }^{19} \mathrm{~F}\right\}$ NMR (126 MHz, Chloroform-d) $\delta$ 149.6, 134.1, 129.2, 127.0, 125.3, 125.2, 118.9, 117.2, 111.2, 111.1, 110.3, 108.5, 83.6, 36.8, 33.4, 32.0, 30.8, 27.0, 26.8, 26.0, 24.6, 24.5, 21.4, carbon attached to boron atom not observed. ${ }^{19} \mathrm{~F}\left\{{ }^{1} \mathrm{H}\right\}$ NMR $(470 \mathrm{MHz}$, Chloroform- $d)$ $\delta-80.8--80.9(\mathrm{~m}),-114.2--114.4(\mathrm{~m}),-121.8--122.1(\mathrm{~m}),-122.8--123.0(\mathrm{~m}),-$ $123.1--123.3(\mathrm{~m}),-126.1--126.2(\mathrm{~m}) .{ }^{11} \mathrm{~B}$ NMR $\left(96 \mathrm{MHz}, \mathrm{CDCl}_{3}\right) \delta 34.8$. HRMS (EI) $\mathrm{m} / \mathrm{z}:\left[\mathrm{M}-\mathrm{CH}_{3}\right]^{+}$calcd. for $\mathrm{C}_{27} \mathrm{H}_{33} \mathrm{O}_{2} \mathrm{BClF}_{13}{ }^{+}$682.2054; found 682.2066. FTIR (neat): $v\left(\mathrm{~cm}^{-1}\right)$ 2958, 1565, 1467, 1321, 1235, 1201, 1149, 961, 910, 846, 792, 703, 658.

2-(2-(3-Bromophenyl)-8,8,9,9,10,10,11,11,12,12,13,13,13-tridecafluoro-5,5dimethyltridecan-2-yl)-4,4,5,5-tetramethyl-1,3,2-dioxaborolane (6q)

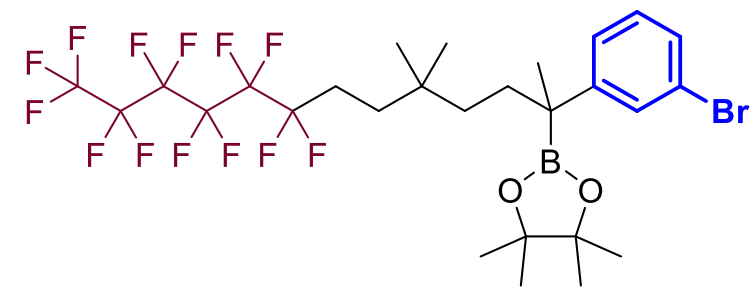

Following GP2 using 2-(5,5-dimethylhept-6-en-2-yl)-4,4,5,5-tetramethyl-1,3,2dioxaborolane (4a, $60 \mu \mathrm{L}, 50.4 \mathrm{mg}, 0.20 \mathrm{mmol}), 1,3$-dibromobenzene (51.9 mg, 0.22 mmol) and 1-bromoperfluorohexane (64 $\mu \mathrm{L}, 119.7 \mathrm{mg}, 0.3 \mathrm{mmol})$, flash chromatography eluting with pentane/DCM (4:1) afforded $\mathbf{6 q}$ as colorless sticky oil (77.5 mg, 53\% yield). $\mathrm{R}_{\mathrm{f}} 0.4$ (pentane/DCM = 4:1). ${ }^{1} \mathrm{H}$ NMR (300 MHz, Chloroformd) $\delta 7.41-7.34(\mathrm{~m}, 1 \mathrm{H}), 7.24-7.12(\mathrm{~m}, 2 \mathrm{H}), 7.11-6.99(\mathrm{~m}, 1 \mathrm{H}), 2.06-1.75(\mathrm{~m}, 2 \mathrm{H})$, $1.72-1.58(\mathrm{~m}, 1 \mathrm{H}), 1.56-1.45(\mathrm{~m}, 1 \mathrm{H}), 1.45-1.34(\mathrm{~m}, 2 \mathrm{H}), 1.22(\mathrm{~s}, 3 \mathrm{H}), 1.13(\mathrm{~s}$, 6H), $1.12(\mathrm{~s}, 6 \mathrm{H}), 1.08-0.98(\mathrm{~m}, 2 \mathrm{H}), 0.79(\mathrm{~s}, 3 \mathrm{H}), 0.79(\mathrm{~s}, 3 \mathrm{H}) .{ }^{13} \mathrm{C}\left\{{ }^{1} \mathrm{H},{ }^{19} \mathrm{~F}\right\} \mathrm{NMR}$ (126 MHz, Chloroform- $d$ ) $\delta 149.9,129.9,129.6,128.3,125.6,122.5,118.9,117.2$, 111.2, 111.1, 110.3, 108.5, 83.6, 36.8, 33.4, 32.0, 30.8, 27.0, 26.8, 26.0, 24.6, 24.5, 21.4, carbon attached to boron atom not observed. ${ }^{19} \mathrm{~F}\left\{{ }^{1} \mathrm{H}\right\}$ NMR $(470 \mathrm{MHz}$, Chloroform- $d)$ $\delta-80.8--80.9(\mathrm{~m}),-114.2--114.4(\mathrm{~m}),-121.7--122.2(\mathrm{~m}),-122.7--123.0(\mathrm{~m}),-$ $123.1--123.4(\mathrm{~m}),-126.0--126.3(\mathrm{~m}) .{ }^{11} \mathrm{~B} \mathrm{NMR}\left(96 \mathrm{MHz}, \mathrm{CDCl}_{3}\right) \delta 33.9 . \mathbf{H R M S}$ (ESI) $m / z:[\mathrm{M}+\mathrm{Na}]^{+}$calcd. for $\mathrm{C}_{27} \mathrm{H}_{33} \mathrm{O}_{2} \mathrm{BBrF}_{13} \mathrm{Na}^{+} 749.1442$; found 749.1450. FTIR (neat): $v\left(\mathrm{~cm}^{-1}\right)$ 2962, 1561, 1472, 1349, 1317, 1236, 1200, 1142, 1069, 1020, 966, 915 , $847,784,705,652$.

4,4,5,5-Tetramethyl-2-(8,8,9,9,10,10,11,11,12,12,13,13,13-tridecafluoro-2-(3iodophenyl)-5,5-dimethyltridecan-2-yl)-1,3,2-dioxaborolane (6r) 


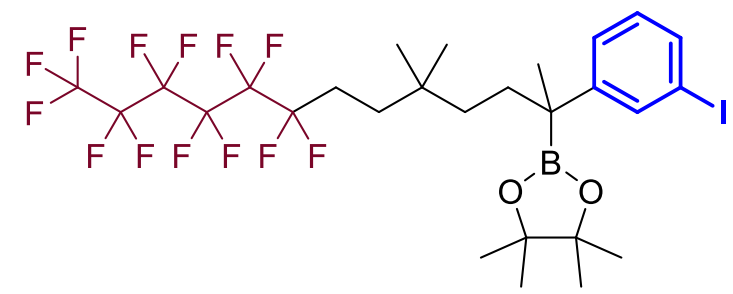

Following GP2 using 2-(5,5-dimethylhept-6-en-2-yl)-4,4,5,5-tetramethyl-1,3,2dioxaborolane (4a, $60 \mu \mathrm{L}, 50.4 \mathrm{mg}, 0.20 \mathrm{mmol}$ ), 1,3-diiodobenzene (72.6 mg, 0.22 mmol) and 1-bromoperfluorohexane (64 $\mu \mathrm{L}, 119.7 \mathrm{mg}, 0.3 \mathrm{mmol})$, flash chromatography eluting with pentane/DCM (4:1) afforded 6r as colorless sticky oil (97.4 mg, 63\% yield). $\mathrm{R}_{\mathrm{f}} 0.4$ (pentane/DCM = 4:1). ${ }^{1} \mathrm{H}$ NMR (300 MHz, Chloroformd) $\delta 7.59-7.54(\mathrm{~m}, 1 \mathrm{H}), 7.44-7.36(\mathrm{~m}, 1 \mathrm{H}), 7.24-7.17(\mathrm{~m}, 1 \mathrm{H}), 6.92(\mathrm{t}, J=7.8 \mathrm{~Hz}$, $1 \mathrm{H}), 2.06-1.74(\mathrm{~m}, 2 \mathrm{H}), 1.70-1.57(\mathrm{~m}, 1 \mathrm{H}), 1.54-1.44(\mathrm{~m}, 1 \mathrm{H}), 1.44-1.34(\mathrm{~m}$, 2H), $1.21(\mathrm{~s}, 3 \mathrm{H}), 1.13(\mathrm{~s}, 6 \mathrm{H}), 1.12(\mathrm{~s}, 6 \mathrm{H}), 1.08-0.97(\mathrm{~m}, 2 \mathrm{H}), 0.79(\mathrm{~s}, 3 \mathrm{H}), 0.79$ (s, $3 \mathrm{H}) .{ }^{13} \mathrm{C}\left\{{ }^{1} \mathrm{H},{ }^{19} \mathrm{~F}\right\}$ NMR $(126 \mathrm{MHz}$, Chloroform- $d$ ) $\delta 150.0,136.0,134.3,129.8,126.2$, 118.9, 117.2, 111.2, 111.1, 110.3, 108.5, 94.7, 83.6, 36.9, 33.4, 32.0, 30.8, 27.0, 26.8, 26.0, 24.6, 24.5, 21.4, carbon attached to boron atom not observed. ${ }^{19} \mathrm{~F}\left\{{ }^{1} \mathrm{H}\right\} \mathrm{NMR}(470$ $\mathrm{MHz}$, Chloroform- $d$ ) $\delta-80.8--80.9(\mathrm{~m}),-114.2--114.4(\mathrm{~m}),-121.8--122.1(\mathrm{~m}),-$ $122.7--123.1(\mathrm{~m}),-123.1--123.3(\mathrm{~m}),-126.1--126.3(\mathrm{~m}) .{ }^{11} \mathrm{~B}$ NMR $(96 \mathrm{MHz}$, $\left.\mathrm{CDCl}_{3}\right) \delta$ 34.4. HRMS (ESI) $m / z:[\mathrm{M}+\mathrm{H}]^{+}$calcd. for $\mathrm{C}_{27} \mathrm{H}_{33} \mathrm{O}_{2} \mathrm{BF}_{13} \mathrm{IH}^{+} 775.1488$, found 775.1494. FTIR (neat): $v\left(\mathrm{~cm}^{-1}\right)$ 2962, 1557, 1470, 1349, 1317, 1237, 1203, 1142, 1069, 1020, 966, 908, 846, 809, 733, 652.

4,4,5,5-Tetramethyl-2-(8,8,9,9,10,10,11,11,12,12,13,13,13-tridecafluoro-5,5dimethyl-2-(m-tolyl)tridecan-2-yl)-1,3,2-dioxaborolane (6s)

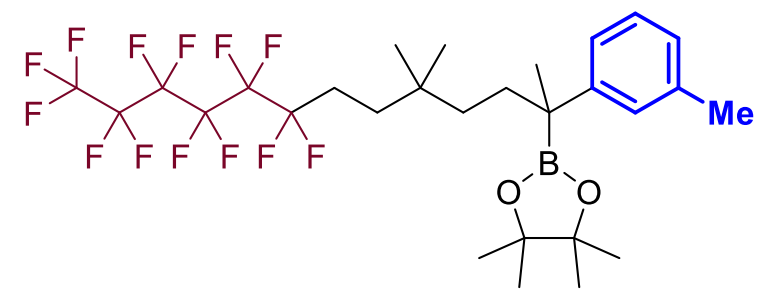

Following GP2 using 2-(5,5-dimethylhept-6-en-2-yl)-4,4,5,5-tetramethyl-1,3,2dioxaborolane (4a, $60 \mu \mathrm{L}, 50.4 \mathrm{mg}, 0.20 \mathrm{mmol}$ ), 3-methyliodobenzene (48.0 mg, 0.22 mmol) and 1-bromoperfluorohexane (64 $\mu \mathrm{L}, 119.7 \mathrm{mg}, 0.3 \mathrm{mmol})$, flash chromatography eluting with pentane/DCM (4:1) afforded $\mathbf{6 s}$ as colorless sticky oil (74.0 mg, 56\% yield). $\mathrm{R}_{\mathrm{f}} 0.4$ (pentane/DCM = 4:1). ${ }^{1} \mathrm{H}$ NMR (400 MHz, Chloroformd) $\delta 7.12-7.06(\mathrm{~m}, 1 \mathrm{H}), 7.06-7.00(\mathrm{~m}, 2 \mathrm{H}), 6.90-6.85(\mathrm{~m}, 1 \mathrm{H}), 2.25(\mathrm{~s}, 3 \mathrm{H}), 2.04-$ $1.74(\mathrm{~m}, 1 \mathrm{H}), 1.74-1.62(\mathrm{~m}, 1 \mathrm{H}), 1.53-1.42(\mathrm{~m}, 1 \mathrm{H}), 1.42-1.35(\mathrm{~m}, 2 \mathrm{H}), 1.23(\mathrm{~s}$, $3 \mathrm{H}), 1.13(\mathrm{~s}, 6 \mathrm{H}), 1.12(\mathrm{~s}, 6 \mathrm{H}), 1.08-1.01(\mathrm{~m}, 2 \mathrm{H}), 0.80(\mathrm{~s}, 3 \mathrm{H}), 0.79(\mathrm{~s}, 3 \mathrm{H}) .{ }^{13} \mathrm{C}\left\{{ }^{1} \mathrm{H}\right.$, ${ }^{19} \mathrm{~F}$ \} NMR (126 MHz, Chloroform- $d$ ) $\delta 147.3,137.4,127.9,127.5,125.9,123.9,119.0$, 117.3, 111.2, 111.1, 110.3, 108.5, 83.3, 36.8, 33.5, 32.0, 30.7, 27.1, 27.0, 26.1, 24.6, $24.5,21.6,21.6$, carbon attached to boron atom not observed. ${ }^{19} \mathrm{~F}\left\{{ }^{1} \mathrm{H}\right\} \mathrm{NMR}(470 \mathrm{MHz}$, Chloroform- $d$ ) $\delta$-80.8 - -80.9 (m), -114.2 - -114.4 (m), -121.8 - -122.1 (m), -122.8 - $123.0(\mathrm{~m}),-123.1--123.3(\mathrm{~m}),-126.0--126.3(\mathrm{~m}) .{ }^{11} \mathrm{~B}$ NMR $\left(128 \mathrm{MHz}, \mathrm{CDCl}_{3}\right) \delta$ 
33.3. HRMS (ESI) $\mathrm{m} / \mathrm{z}$ : $[\mathrm{M}+\mathrm{Na}]^{+}$calcd. for $\mathrm{C}_{28} \mathrm{H}_{36} \mathrm{O}_{2} \mathrm{BF}_{13} \mathrm{Na}^{+}$685.2498; found 685.2499. FTIR (neat): $v\left(\mathrm{~cm}^{-1}\right)$ 2961, 1472, 1348, 1313, 1238, 1205, 1144, 1069, 967 , $913,856,785,709,651$.

4,4,5,5-Tetramethyl-2-(8,8,9,9,10,10,11,11,12,12,13,13,13-tridecafluoro-5,5dimethyl-2-(o-tolyl)tridecan-2-yl)-1,3,2-dioxaborolane (6t)

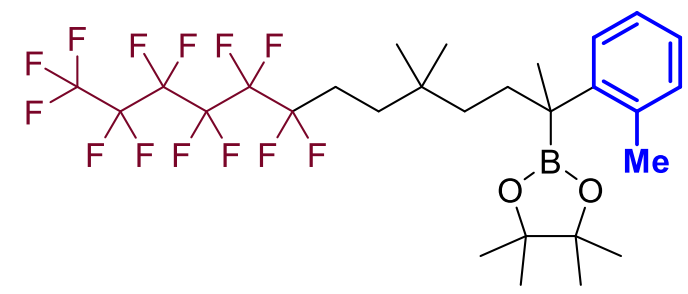

Following GP2 using 2-(5,5-dimethylhept-6-en-2-yl)-4,4,5,5-tetramethyl-1,3,2dioxaborolane (4a, $60 \mu \mathrm{L}, 50.4 \mathrm{mg}, 0.20 \mathrm{mmol}$ ), 2-methyliodobenzene (48.0 mg, 0.22 mmol) and 1-bromoperfluorohexane $(64 \mu \mathrm{L}, 119.7 \mathrm{mg}, 0.3 \mathrm{mmol})$, flash chromatography eluting with pentane/DCM (4:1) afforded 6t as colorless sticky oil (66.6 mg, 50\% yield). $\mathrm{R}_{\mathrm{f}} 0.4$ (pentane/DCM = 4:1). ${ }^{1} \mathrm{H}$ NMR (400 MHz, Chloroformd) $\delta 7.15-6.93(\mathrm{~m}, 5 \mathrm{H}), 2.24(\mathrm{~s}, 3 \mathrm{H}), 1.93-1.54(\mathrm{~m}, 4 \mathrm{H}), 1.37-1.29(\mathrm{~m}, 2 \mathrm{H}), 1.26$ (s, 3H), 1.17 (s, 12H), $1.13-0.99(\mathrm{~m}, 1 \mathrm{H}), 0.75$ (s, 3H), 0.73 (s, 3H), $0.71-0.55$ (m, 1H). ${ }^{13} \mathrm{C}\left\{{ }^{1} \mathrm{H},{ }^{19} \mathrm{~F}\right\}$ NMR $(126 \mathrm{MHz}$, Chloroform- $d$ ) $\delta$ 144.4, 135.7, 130.9, 126.4, 125.6, 125.4, 118.9, 117.2, 111.1, 111.1, 110.3, 108.5, 83.3, 34.8, 31.8, 30.5, 30.3, 27.0, 27.0, $25.9,24.9,24.8,21.8,21.0$, carbon attached to boron atom not observed. ${ }^{19} \mathrm{~F}\left\{{ }^{1} \mathrm{H}\right\} \mathrm{NMR}$ $(470 \mathrm{MHz}$, Chloroform- $d$ ) $\delta-80.8--80.9(\mathrm{~m}),-114.4--114.6(\mathrm{~m}),-121.8--122.1(\mathrm{~m})$, $-122.8--123.0(\mathrm{~m}),-123.2--123.4(\mathrm{~m}),-126.1--126.3(\mathrm{~m}) .{ }^{11} \mathrm{~B}$ NMR $(128 \mathrm{MHz}$, $\left.\mathrm{CDCl}_{3}\right) \delta$ 34.9. HRMS (ESI) $\mathrm{m} / z$ : $[\mathrm{M}+\mathrm{Na}]^{+}$calcd. for $\mathrm{C}_{28} \mathrm{H}_{36} \mathrm{O}_{2} \mathrm{BF}_{13} \mathrm{Na}^{+}$685.2498; found 685.2500. FTIR (neat): $v\left(\mathrm{~cm}^{-1}\right)$ 2961, 2874, 1472, 1373, 1343, 1312, 1238, 1205, 1144, 1118, 1087, 1046, 966, 907, 848, 812, 728, 707, 651.

2-(2-(4-Bromo-3-methylphenyl)-8,8,9,9,10,10,11,11,12,12,13,13,13-tridecafluoro5,5-dimethyltridecan-2-yl)-4,4,5,5-tetramethyl-1,3,2-dioxaborolane (6u)

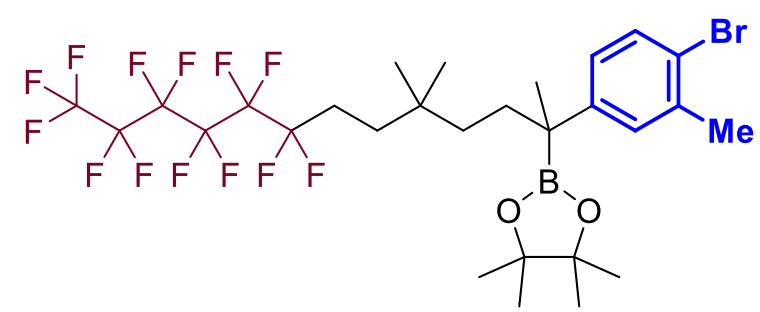

Following GP2 using 2-(5,5-dimethylhept-6-en-2-yl)-4,4,5,5-tetramethyl-1,3,2dioxaborolane (4a, $60 \mu \mathrm{L}, 50.4 \mathrm{mg}, 0.20 \mathrm{mmol})$, 1-iodo-3-methyl-4-bromobenzene (65.3 mg, $0.22 \mathrm{mmol}$ ) and 1-bromoperfluorohexane (64 $\mu \mathrm{L}, 119.7 \mathrm{mg}, 0.3 \mathrm{mmol})$, flash chromatography eluting with pentane/DCM (4:1) afforded $\mathbf{6 u}$ as colorless sticky oil (86.1 mg, 58\% yield). $\mathrm{R}_{\mathrm{f}} 0.4$ (pentane/DCM = 4:1). ${ }^{1} \mathrm{H}$ NMR (400 MHz, Chloroformd) $\delta 7.33(\mathrm{~d}, J=8.3 \mathrm{~Hz}, 1 \mathrm{H}), 7.11-7.05(\mathrm{~m}, 1 \mathrm{H}), 6.92(\mathrm{dd}, J=8.4,2.4 \mathrm{~Hz}, 1 \mathrm{H}), 2.29$ (s, 3H), $2.07-1.72(\mathrm{~m}, 2 \mathrm{H}), 1.69-1.58(\mathrm{~m}, 1 \mathrm{H}), 1.52-1.33(\mathrm{~m}, 3 \mathrm{H}), 1.21(\mathrm{~s}, 3 \mathrm{H})$, $1.13(\mathrm{~s}, 6 \mathrm{H}), 1.12$ (s, 6H), $1.07-0.97(\mathrm{~m}, 2 \mathrm{H}), 0.79(\mathrm{~s}, 3 \mathrm{H}), 0.78(\mathrm{~s}, 3 \mathrm{H}) .{ }^{13} \mathrm{C}\left\{{ }^{1} \mathrm{H},{ }^{19} \mathrm{~F}\right\}$ 
NMR (126 MHz, Chloroform-d) $\delta 146.6,137.2,131.9,129.3,126.1,121.5,118.9$, $117.2,111.2,111.1,110.3,108.5,83.5,36.8,33.4,32.0,30.7,27.1,26.9,26.0,24.6$, 24.5, 23.0, 21.5, carbon attached to boron atom not observed. ${ }^{19} \mathrm{~F}\left\{{ }^{1} \mathrm{H}\right\} \mathrm{NMR}(564 \mathrm{MHz}$, Chloroform- $d$ ) $\delta$-80.8 - -80.9 (m), -114.3 - -114.4 (m), -121.9- -122.1 (m), -122.8 - $123.0(\mathrm{~m}),-123.1--123.3(\mathrm{~m}),-126.1--126.3(\mathrm{~m}) .{ }^{11} \mathrm{~B}$ NMR $\left(128 \mathrm{MHz}, \mathrm{CDCl}_{3}\right) \delta$ 32.9. HRMS (ESI) $\mathrm{m} / z$ : $[\mathrm{M}+\mathrm{Na}]^{+}$calcd. for $\mathrm{C}_{87} \mathrm{H}_{35} \mathrm{O}_{2} \mathrm{BBrF}_{13} \mathrm{Na}^{+} 763.1603$; found 763.1606. FTIR (neat): $v\left(\mathrm{~cm}^{-1}\right)$ 2978, 2871, 1473, 1348, 1314, 1238, 1205, 1143, 1120, $1027,907,856,815,731,650$.

2-(2-(3,5-Dimethylphenyl)-8,8,9,9,10,10,11,11,12,12,13,13,13-tridecafluoro-5,5dimethyltridecan-2-yl)-4,4,5,5-tetramethyl-1,3,2-dioxaborolane (6v)

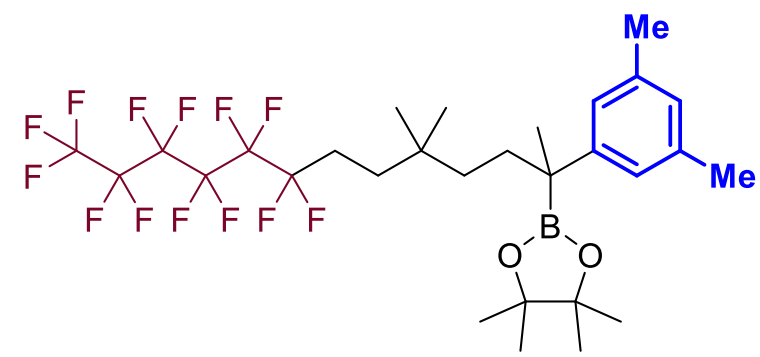

Following GP2 using 2-(5,5-dimethylhept-6-en-2-yl)-4,4,5,5-tetramethyl-1,3,2dioxaborolane (4a, $60 \mu \mathrm{L}, 50.4 \mathrm{mg}, 0.20 \mathrm{mmol}$ ), 3,5-dimethyliodobenzene (51.0 mg, $0.22 \mathrm{mmol}$ ) and 1-bromoperfluorohexane $(64 \mu \mathrm{L}, 119.7 \mathrm{mg}, 0.3 \mathrm{mmol})$, flash chromatography eluting with pentane/DCM (4:1) afforded $\mathbf{6 v}$ as colorless sticky oil (77.2 mg, 57\% yield). $\mathrm{R}_{\mathrm{f}} 0.4$ (pentane/DCM = 4:1). ${ }^{1} \mathrm{H}$ NMR $(500 \mathrm{MHz}$, Chloroformd) $\delta 6.94(\mathrm{~s}, 2 \mathrm{H}), 6.80(\mathrm{~s}, 1 \mathrm{H}), 2.31(\mathrm{~s}, 3 \mathrm{H}), 2.10-2.01(\mathrm{~m}, 1 \mathrm{H}), 2.00-1.91(\mathrm{~m}, 1 \mathrm{H})$, $1.83-1.73(\mathrm{~m}, 1 \mathrm{H}), 1.62-1.45(\mathrm{~m}, 3 \mathrm{H}), 1.32(\mathrm{~s}, 3 \mathrm{H}), 1.24(\mathrm{~s}, 6 \mathrm{H}), 1.23(\mathrm{~s}, 6 \mathrm{H}), 1.20$ $-1.13(\mathrm{~m}, 2 \mathrm{H}), 0.91(\mathrm{~s}, 3 \mathrm{H}), 0.90(\mathrm{~s}, 3 \mathrm{H}) .{ }^{13} \mathrm{C}\left\{{ }^{1} \mathrm{H},{ }^{19} \mathrm{~F}\right\}(126 \mathrm{MHz}$, Chloroform- $d) \delta$ 147.3, 137.3, 126.9, 124.6, 119.0, 117.3, 111.2, 111.1, 110.3, 108.5, 83.3, 37.0, 33.5, $32.0,30.8,27.1,27.0,26.1,24.6,24.5,21.7,21.5$, carbon attached to boron atom not observed. ${ }^{19} \mathrm{~F}\left\{{ }^{1} \mathrm{H}\right\}$ NMR $(470 \mathrm{MHz}$, Chloroform- $d) \delta-80.8--80.9(\mathrm{~m}),-114.1--114.4$ (m), -121.8--122.2 (m), -122.8--123.0 (m), -123.1--123.3 (m), -126.0--126.3 (m). ${ }^{11} \mathrm{~B}$ NMR $\left(128 \mathrm{MHz}, \mathrm{CDCl}_{3}\right) \delta$ 32.8. HRMS (ESI) $\mathrm{m} / \mathrm{z}:[\mathrm{M}+\mathrm{Na}]^{+}$calcd. for $\mathrm{C}_{29} \mathrm{H}_{38} \mathrm{O}_{2} \mathrm{BF}_{13} \mathrm{Na}^{+}$699.2655; found 699.2655. FTIR (neat): $v\left(\mathrm{~cm}^{-1}\right)$ 2928, 1693, 1659, $1551,1531,1513,1465,1313,1234,1199,961,912,851,706,647$.

2-(8,8,9,9,10,10,11,11,12,12,13,13,14,14,15,15,15-Heptadecafluoro-2,5,5trimethylpentadecan-2-yl)-4,4,5,5-tetramethyl-1,3,2-dioxaborolane (6w)

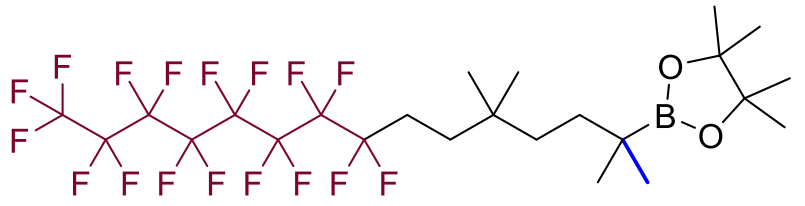

Following GP1 using 2-(5,5-dimethylhept-6-en-2-yl)-4,4,5,5-tetramethyl-1,3,2dioxaborolane $(\mathbf{4 a}, 60 \mu \mathrm{L}, 50.4 \mathrm{mg}, 0.20 \mathrm{mmol})$, methyl lithium $(0.22 \mathrm{~mL}, 1.0 \mathrm{M}$ in $\left.\mathrm{Et}_{2} \mathrm{O}\right)$ and 1-bromoperfluorooctane $(77.6 \mu \mathrm{L}, 149.7 \mathrm{mg}, 0.3 \mathrm{mmol})$, flash chromatography eluting with pentane/DCM (4:1) afforded $\mathbf{6 w}$ as colorless sticky oil 
(83.5 mg, 61\% yield). $\mathrm{R}_{\mathrm{f}} 0.4$ (pentane/DCM = 4:1). ${ }^{1} \mathrm{H}$ NMR (300 MHz, Chloroformd) $\delta 2.09-1.76(\mathrm{~m}, 2 \mathrm{H}), 1.46-1.31(\mathrm{~m}, 2 \mathrm{H}), 1.14(\mathrm{~s}, 12 \mathrm{H}), 1.13-0.98(\mathrm{~m}, 4 \mathrm{H}), 0.84$ $(\mathrm{s}, 6 \mathrm{H}), 0.80(\mathrm{~s}, 6 \mathrm{H}) .{ }^{13} \mathrm{C}\left\{{ }^{1} \mathrm{H},{ }^{19} \mathrm{~F}\right\}$ NMR $(151 \mathrm{MHz}$, Chloroform- $d) \delta$ 119.0, 117.2, 111.2, 110.9, 110.8, 110.3, 108.4, 82.9, 37.9, 35.0, 31.9, 30.9, 27.0, 26.1, 25.0, 24.6, carbon attached to boron atom not observed. ${ }^{19} \mathrm{~F}\left\{{ }^{1} \mathrm{H}\right\}$ NMR $(564 \mathrm{MHz}$, Chloroform- $d)$ $\delta-80.9--81.0(\mathrm{~m}),-114.4--114.5(\mathrm{~m}),-121.7--121.9(\mathrm{~m}),-121.9--122.1(\mathrm{~m}),-$ 122.7 - -122.9 (m), -123.2 - -123.4 (m), -126.1 - -126.3 (m). ${ }^{11} \mathrm{~B}$ NMR (96 MHz, $\left.\mathrm{CDCl}_{3}\right) \delta$ 33.7. HRMS (ESI) $\mathrm{m} / z$ : $[\mathrm{M}+\mathrm{Na}]^{+}$calcd. for $\mathrm{C}_{24} \mathrm{H}_{32} \mathrm{O}_{2} \mathrm{BF}_{17} \mathrm{Na}^{+}$709.2120; found 709.2124. FTIR (neat): $v\left(\mathrm{~cm}^{-1}\right)$ 2980, 2961, 2870, 1477, 1372, 1309, 1239, 1202 , 1145, 1113, 1081, 1024, 968, 854, 737, 654.

5,5,8-Trimethyl-8-(4,4,5,5-tetramethyl-1,3,2-dioxaborolan-2-yl)nonanenitrile (6x)

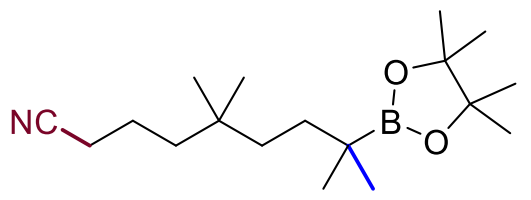

Following GP1 using 2-(5,5-dimethylhept-6-en-2-yl)-4,4,5,5-tetramethyl-1,3,2dioxaborolane (4a, $60 \mu \mathrm{L}, 50.4 \mathrm{mg}, 0.20 \mathrm{mmol})$, methyl lithium $(0.22 \mathrm{~mL}, 1.0 \mathrm{M}$ in $\left.\mathrm{Et}_{2} \mathrm{O}\right)$ and ethyl bromoacetonitrile $(36.0 \mathrm{mg}, 0.3 \mathrm{mmol})$, flash chromatography eluting with pentane/Et ${ }_{2} \mathrm{O}$ (4:1) afforded $\mathbf{6 x}$ as colorless sticky oil (10.2 mg, 17\% yield). $\mathrm{R}_{\mathrm{f}} 0.3$ (pentane/Et $\left.{ }_{2} \mathrm{O}=4: 1\right) .{ }^{1} \mathrm{H}$ NMR $(300 \mathrm{MHz}$, Chloroform- $d) \delta 2.30(\mathrm{t}, J=7.2 \mathrm{~Hz}, 2 \mathrm{H})$, $1.68-1.54(\mathrm{~m}, 2 \mathrm{H}), 1.33-1.25(\mathrm{~m}, 2 \mathrm{H}), 1.22(\mathrm{~s}, 12 \mathrm{H}), 1.19-1.06(\mathrm{~m}, 4 \mathrm{H}), 0.90(\mathrm{~s}$, $6 \mathrm{H}), 0.84(\mathrm{~s}, 6 \mathrm{H}) .{ }^{13} \mathrm{C} \mathrm{NMR}\left(76 \mathrm{MHz}, \mathrm{CDCl}_{3}\right) \delta 119.9,82.9,40.8,38.1,35.1,32.5$, 27.2, 25.0, 24.7, 20.5, 18.0. ${ }^{11} \mathrm{~B}$ NMR $\left(96 \mathrm{MHz}, \mathrm{CDCl}_{3}\right) \delta 34.2$. HRMS (ESI) $\mathrm{m} / \mathrm{z}$ : $[\mathrm{M}+\mathrm{Na}]^{+}$calcd. for $\mathrm{C}_{18} \mathrm{H}_{34} \mathrm{NO}_{2} \mathrm{BNa}^{+} 330.2578$; found 330.2574 . FTIR (neat): $v$ ( $\left.\mathrm{cm}^{-1}\right)$ 2958, 2935, 2862, 1475, 1389, 1371, 1308, 1249, 1166, 1143, 1109, 967, 853, 692.

4,4,5,5-Tetramethyl-2-(8,8,9,9,10,10,11,11,12,12,13,13,13-tridecafluoro-2phenyltridecan-2-yl)-1,3,2-dioxaborolane (6y)

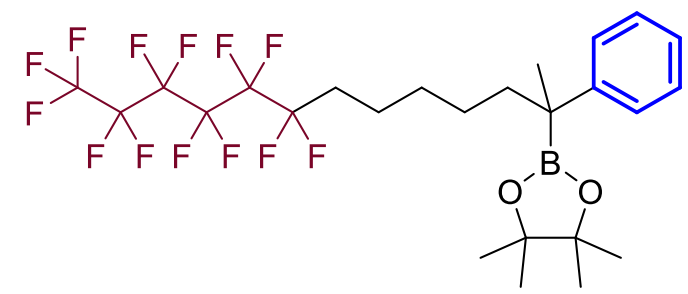

Following GP1 using 2-(hept-6-en-2-yl)-4,4,5,5-tetramethyl-1,3,2-dioxaborolane (4y, $44.8 \mathrm{mg}, 0.20 \mathrm{mmol})$, phenyl lithium $\left(0.12 \mathrm{~mL}, 1.9 \mathrm{M}\right.$ in $\left.{ }^{n} \mathrm{Bu}_{2} \mathrm{O}\right)$ and 1bromoperfluorohexane $(64 \mu \mathrm{L}, 119.7 \mathrm{mg}, 0.3 \mathrm{mmol})$, flash chromatography eluting with pentane/DCM (4:1) afforded $\mathbf{6 y}$ as colorless sticky oil (15.8 mg, 13\% yield). $\mathrm{R}_{\mathrm{f}}$ 0.4 (pentane/DCM = 4:1). ${ }^{1} \mathrm{H}$ NMR $(500 \mathrm{MHz}$, Chloroform- $d) \delta 7.26-7.17(\mathrm{~m}, 4 \mathrm{H})$, $7.10-7.03(\mathrm{~m}, 1 \mathrm{H}), 1.97-1.89(\mathrm{~m}, 2 \mathrm{H}), 1.76-1.68(\mathrm{~m}, 1 \mathrm{H}), 1.65-1.57(\mathrm{~m}, 1 \mathrm{H})$, $1.54-1.45(\mathrm{~m}, 3 \mathrm{H}), 1.32-1.24(\mathrm{~m}, 1 \mathrm{H}), 1.26(\mathrm{~s}, 3 \mathrm{H}), 1.21-1.14(\mathrm{~m}, 2 \mathrm{H}), 1.13(\mathrm{~s}$, $6 \mathrm{H}), 1.12(\mathrm{~s}, 6 \mathrm{H}) .{ }^{13} \mathrm{C}\left\{{ }^{1} \mathrm{H},{ }^{19} \mathrm{~F}\right\}$ NMR (126 MHz, Chloroform- $d$ ) $\delta$ 147.1, 128.1, 126.8, 125.1, 118.5, 117.2, 111.1, 110.3, 108.5, 83.3, 39.1, 30.8, 29.7, 25.1, 24.5, 24.5, 21.5, 
19.9, carbon attached to boron atom not observed. ${ }^{19} \mathrm{~F}\left\{{ }^{1} \mathrm{H}\right\}$ NMR (470 MHz, Chloroform- $d$ ) $\delta$-80.8 - -80.9 (m), -114.3 - -114.4 (m), -121.8 - -122.1 (m), -122.7 - $123.1(\mathrm{~m}),-123.5--123.7(\mathrm{~m}),-126.0--126.2(\mathrm{~m}) .{ }^{11} \mathrm{~B} \mathrm{NMR}\left(96 \mathrm{MHz}, \mathrm{CDCl}_{3}\right) \delta 33.0$. HRMS (ESI) $m / z$ : $[\mathrm{M}+\mathrm{Na}]^{+}$calcd. for $\mathrm{C}_{25} \mathrm{H}_{30} \mathrm{O}_{2} \mathrm{BF}_{13} \mathrm{Na}^{+}$643.2028; found 643.2028. FTIR (neat): $v\left(\mathrm{~cm}^{-1}\right)$ 2979, 1381, 1353, 1313, 1239, 1210, 1144, 1030, 912, 735, 698.

2-(1-(4-(Tert-butyl)phenyl)-7,7,8,8,9,9,10,10,11,11,12,12,12-tridecafluoro-4,4dimethyldodecyl)-4,4,5,5-tetramethyl-1,3,2-dioxaborolane (6z)

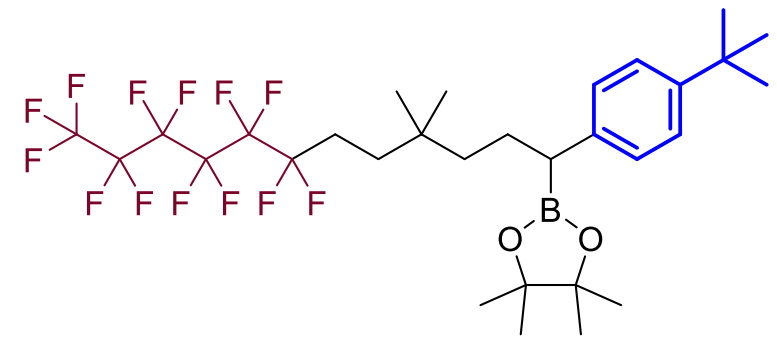

Following GP2 using 2-(4,4-dimethylhex-5-en-1-yl)-4,4,5,5-tetramethyl-1,3,2dioxaborolane (4z, $35.0 \mathrm{mg}, 0.147 \mathrm{mmol})$, 4-tert-butyliodobenzene (52.0 mg, 0.20 mmol, 1.36 equiv), ${ }^{n} \mathrm{BuLi}(0.125 \mathrm{~mL}, 1.6 \mathrm{M}$ in hexane, $0.2 \mathrm{mmol}, 1.36$ equiv) and 1 bromoperfluorohexane (75.8 $\mathrm{mg}, 0.19 \mathrm{mmol}, 1.5$ equiv), flash chromatography eluting with pentane/DCM (3:1) afforded $\mathbf{6 z}$ as colorless sticky oil (32.9 mg, 32\% yield). $\mathbf{R}_{\mathrm{f}}$ 0.4 (pentane/DCM = 3:1). ${ }^{1} \mathrm{H}$ NMR (599 MHz, Chloroform- $\left.d\right) \delta 7.20-7.17(\mathrm{~m}, 2 \mathrm{H})$, $7.06-7.03(\mathrm{~m}, 2 \mathrm{H}), 2.09(\mathrm{dd}, J=8.9,6.7 \mathrm{~Hz}, 1 \mathrm{H}), 1.97-1.88(\mathrm{~m}, 1 \mathrm{H}), 1.87-1.77$ $(\mathrm{m}, 1 \mathrm{H}), 1.73-1.64(\mathrm{~m}, 1 \mathrm{H}), 1.52-1.45(\mathrm{~m}, 1 \mathrm{H}), 1.42-1.35(\mathrm{~m}, 2 \mathrm{H}), 1.22(\mathrm{~s}, 9 \mathrm{H})$, $1.19-1.07(\mathrm{~m}, 2 \mathrm{H}) 1.14(\mathrm{~s}, 6 \mathrm{H}), 1.11(\mathrm{~s}, 6 \mathrm{H}), 0.80(\mathrm{~s}, 3 \mathrm{H}), 0.79(\mathrm{~s}, 3 \mathrm{H}) .{ }^{13} \mathrm{C}\left\{{ }^{1} \mathrm{H},{ }^{19} \mathrm{~F}\right\}$ NMR (151 MHz, Chloroform- $d$ ) $\delta 147.9,140.0,127.8,125.2,118.9,117.2,111.1,110.3$, 108.5, 83.3, 40.9, 34.2, 32.1, 31.4, 30.8, 27.3, 27.1, 27.0, 26.0, 24.7, 24.5, carbon attached to boron atom not observed. ${ }^{19} \mathrm{~F}\left\{{ }^{1} \mathrm{H}\right\} \mathrm{NMR}(564 \mathrm{MHz}$, Chloroform- $d) \delta-80.8$ - -80.9 (m), -114.4 - -114.5 (m), -121.9 - -122.1 (m), -122.8 - -123.0 (m), -123.1 - $123.4(\mathrm{~m}),-126.1--126.3(\mathrm{~m}) .{ }^{11} \mathrm{~B}$ NMR (192 MHz, Chloroform- $d$ ) $\delta 33.8$. HRMS (ESI) $m / z:[\mathrm{M}+\mathrm{Na}]^{+}$calcd. for $\mathrm{C}_{30} \mathrm{H}_{40} \mathrm{O}_{2} \mathrm{BF}_{13} \mathrm{Na}^{+}$713.2811; found 713.2812. FTIR (neat): $v\left(\mathrm{~cm}^{-1}\right)$ 2961, 2939, 2876, 1510, 1367, 1321, 1238, 1206, 1144, 1120, 1022 , $967,853,653$.

2-(3-(4-(Tert-butyl)phenyl)-9,9,10,10,11,11,12,12,13,13,14,14,14-tridecafluoro-6,6dimethyltetradecan-3-yl)-4,4,5,5-tetramethyl-1,3,2-dioxaborolane (6aa)

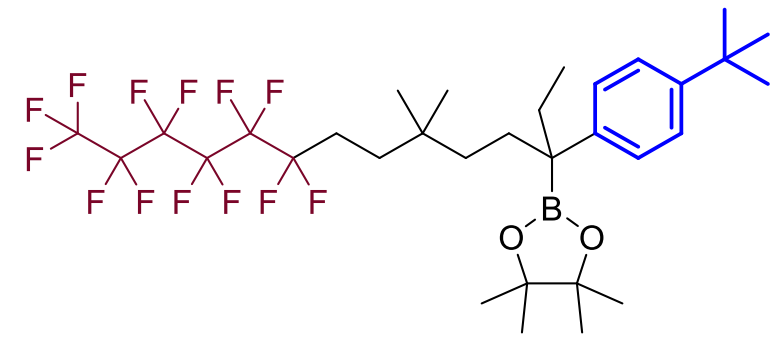

Following GP2 using 2-(6,6-dimethyloct-7-en-3-yl)-4,4,5,5-tetramethyl-1,3,2dioxaborolane (4aa, $53.2 \mathrm{mg}, 0.2 \mathrm{mmol}$ ), 4-tert-butyliodobenzene (57.2 $\mathrm{mg}, 0.22 \mathrm{mmol}$ ) 
and 1-bromoperfluorohexane (75.8 $\mathrm{mg}, 0.19 \mathrm{mmol}, 1.5$ equiv), flash chromatography eluting with pentane/DCM (4:1) afforded 6aa as colorless sticky oil $(62.2 \mathrm{mg}, 43 \%$ yield). $\mathrm{R}_{\mathrm{f}} 0.5$ (pentane/DCM = 4:1). ${ }^{1} \mathrm{H}$ NMR (500 MHz, Chloroform- $d$ ) $\delta 7.31-7.28$ $(\mathrm{m}, 2 \mathrm{H}), 7.24-7.19(\mathrm{~m}, 2 \mathrm{H}), 1.97-1.88(\mathrm{~m}, 2 \mathrm{H}), 1.88-1.77(\mathrm{~m}, 2 \mathrm{H}), 1.74(\mathrm{dd}, J=$ 9.4, $7.7 \mathrm{~Hz}, 2 \mathrm{H}), 1.51-1.46(\mathrm{~m}, 2 \mathrm{H}), 1.31(\mathrm{~s}, 9 \mathrm{H}), 1.24(\mathrm{~s}, 12 \mathrm{H}), 1.12-0.99(\mathrm{~m}, 2 \mathrm{H})$, $0.89(\mathrm{~s}, 3 \mathrm{H}), 0.88(\mathrm{~s}, 3 \mathrm{H}), 0.74(\mathrm{t}, J=7.5 \mathrm{~Hz}, 3 \mathrm{H}) .{ }^{13} \mathrm{C}\left\{{ }^{1} \mathrm{H},{ }^{19} \mathrm{~F}\right\}$ NMR $(126 \mathrm{MHz}$, Chloroform- $d$ ) $\delta 147.6,142.1,127.1,124.8,119.0,117.2,111.2,111.1,110.3,108.5$, 36.1, 34.2, 32.0, 31.3, 30.6, 28.5, 28.2, 27.1, 27.1, 26.0, 24.7, 24.7, 9.5, carbon attached to boron atom not observed. ${ }^{19} \mathrm{~F}\left\{{ }^{1} \mathrm{H}\right\}$ NMR $(470 \mathrm{MHz}$, Chloroform- $d) \delta-80.8--80.9$ (m), -114.3--114.5 (m), -121.8--122.1 (m), -122.7--123.0 (m), -123.1--123.4 (m), $-126.1--126.3(\mathrm{~m}) .{ }^{11} \mathrm{~B}$ NMR $(160 \mathrm{MHz}$, Chloroform- $d$ ) $\delta 34.1$. HRMS (ESI) $m / z$ : $[\mathrm{M}+\mathrm{Na}]^{+}$calcd. for $\mathrm{C}_{32} \mathrm{H}_{44} \mathrm{O}_{2} \mathrm{BF}_{13} \mathrm{Na}^{+} 741.3125$; found 741.3127. FTIR (neat): $v$ ( $\mathrm{cm}^{-}$ 1) 2960, 2874, 1514, 1367, 1309, 1236, 1203, 1143, 1120, 1022, 966, 855, 829, 811, 707, 694, 651 .

4,4,5,5-Tetramethyl-2-(8,8,8-trifluoro-2,4,4-trimethyloctan-2-yl)-1,3,2-dioxaborolane (9a)

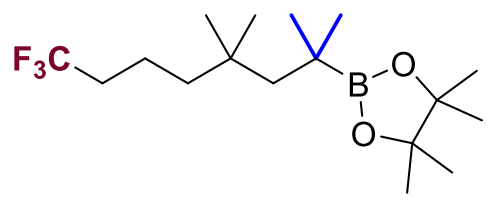

Following GP1 using 2-(2,2-dimethylpent-4-en-1-yl)-4,4,5,5-tetramethyl-1,3,2dioxaborolane (7a, $44.8 \mathrm{mg}, 0.20 \mathrm{mmol})$, isopropyl lithium $(0.31 \mathrm{~mL}, 0.22 \mathrm{mmol}, 0.7$ $\mathrm{M}$ in pentane) and trifluoromethyl iodide $(0.3 \mathrm{~mL}, 0.3 \mathrm{mmol}, 1.0 \mathrm{M}$ in acetonitrile), flash chromatography eluting with pentane/DCM (2:1) afforded 9a as colorless sticky oil (48.5 mg, 72\% yield). $\mathrm{R}_{\mathrm{f}} 0.5$ (pentane/DCM = 2:1). ${ }^{1} \mathrm{H}$ NMR (300 MHz, Chloroform- $d$ ) $\delta 2.05-1.80(\mathrm{~m}, 2 \mathrm{H}), 1.54-1.38(\mathrm{~m}, 2 \mathrm{H}), 1.28(\mathrm{~s}, 2 \mathrm{H}), 1.23-1.10(\mathrm{~m}$, $14 \mathrm{H}), 0.89(\mathrm{~s}, 6 \mathrm{H}), 0.85(\mathrm{~s}, 6 \mathrm{H}) .{ }^{13} \mathrm{C}\left\{{ }^{1} \mathrm{H},{ }^{19} \mathrm{~F}\right\} \mathrm{NMR}(151 \mathrm{MHz}$, Chloroform- $d$ ) $\delta 127.2$, 82.9, 52.3, 44.0, 34.6, 33.9, 28.0, 27.4, 24.7, 16.8, carbon attached to boron atom not observed. ${ }^{19} \mathrm{~F}\left\{{ }^{1} \mathrm{H}\right\}$ NMR $\left(282 \mathrm{MHz}, \mathrm{CDCl}_{3}\right) \delta$-66.4. ${ }^{11} \mathrm{~B} \mathrm{NMR}\left(96 \mathrm{MHz}, \mathrm{CDCl}_{3}\right) \delta 34.7$. HRMS (ESI) $m / z$ : $[\mathrm{M}+\mathrm{Na}]^{+}$calcd. for $\mathrm{C}_{17} \mathrm{H}_{32} \mathrm{O}_{2} \mathrm{BF}_{3} \mathrm{Na}^{+}$359.2343; found 359.2340. FTIR (neat): $v\left(\mathrm{~cm}^{-1}\right) 2959,1481,1389,1371,1306,1283,1253,1136,1080,1023$, $967,847,692,668,632$.

4,4,5,5-Tetramethyl-2-(8,8,9,9,9-pentafluoro-2,4,4-trimethylnonan-2-yl)-1,3,2dioxaborolane (9b)

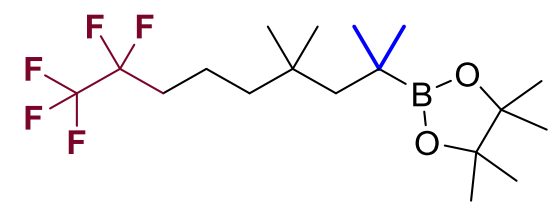

Following GP1 using 2-(2,2-dimethylpent-4-en-1-yl)-4,4,5,5-tetramethyl-1,3,2dioxaborolane (7a, $44.8 \mathrm{mg}, 0.20 \mathrm{mmol})$, isopropyl lithium $(0.31 \mathrm{~mL}, 0.22 \mathrm{mmol}, 0.7$ $\mathrm{M}$ in pentane) and pentafluoroethyl iodide $(0.3 \mathrm{~mL}, 0.3 \mathrm{mmol}, 1.0 \mathrm{M}$ in acetonitrile), flash chromatography eluting with pentane/DCM (2:1) afforded $9 \mathbf{b}$ as colorless sticky 
oil (42.1 mg, 55\% yield). $\mathrm{R}_{\mathrm{f}} 0.6$ (pentane/DCM = 2:1). ${ }^{1} \mathrm{H}$ NMR (300 MHz, Chloroform- $d$ ) $\delta 1.99-1.76(\mathrm{~m}, 2 \mathrm{H}), 1.58-1.40(\mathrm{~m}, 2 \mathrm{H}), 1.29(\mathrm{~s}, 2 \mathrm{H}), 1.22-1.12(\mathrm{~m}$, 14H), 0.89 (s, 6H), $0.86(\mathrm{~s}, 6 \mathrm{H}) .{ }^{13} \mathrm{C}\left\{{ }^{1} \mathrm{H},{ }^{19} \mathrm{~F}\right\}$ NMR (151 MHz, Chloroform- $d$ ) $\delta 119.3$, $115.8,82.9,52.3,44.4,33.9,31.5,28.0,27.4,24.7,15.2$, carbon attached to boron atom not observed. ${ }^{19} \mathrm{~F}\left\{{ }^{1} \mathrm{H}\right\}$ NMR (564 MHz, Chloroform- $d$ ) $\delta-85.5(\mathrm{~s}),-118.2(\mathrm{~s}) .{ }^{11} \mathrm{~B}$ NMR $\left(96 \mathrm{MHz}, \mathrm{CDCl}_{3}\right) \delta$ 34.7. HRMS (ESI) $\mathrm{m} / z$ : $[\mathrm{M}+\mathrm{Na}]^{+}$calcd. for $\mathrm{C}_{18} \mathrm{H}_{32} \mathrm{O}_{2} \mathrm{BF}_{5} \mathrm{Na}^{+}$ 409.2311; found 409.2307. FTIR (neat): $v\left(\mathrm{~cm}^{-1}\right)$ 2960, 2911, 2871, 1481, 1390, 1372 , 1340, 1307, 1267, 1193, 1145, 1067, 846, 714, 692.

2-(8,8,9,9,10,10,10-Heptafluoro-2,4,4-trimethyldecan-2-yl)-4,4,5,5-tetramethyl-1,3,2dioxaborolane $(\mathbf{9 c})$

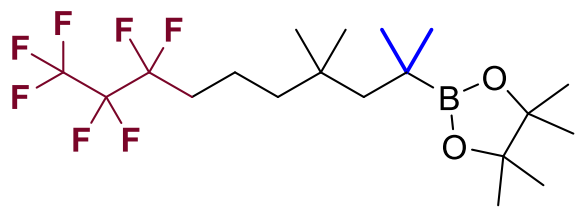

Following GP1 using 2-(2,2-dimethylpent-4-en-1-yl)-4,4,5,5-tetramethyl-1,3,2dioxaborolane (7a, $44.8 \mathrm{mg}, 0.20 \mathrm{mmol})$, isopropyl lithium $(0.31 \mathrm{~mL}, 0.22 \mathrm{mmol}, 0.7$ $\mathrm{M}$ in pentane) and perfluoropropyl iodide ( $88.8 \mathrm{mg}, 0.3 \mathrm{mmol})$, flash chromatography eluting with pentane/DCM (3:1) afforded 9c as colorless sticky oil (67.1 mg, 77\% yield) $\mathrm{R}_{\mathrm{f}} 0.5$ (pentane/DCM = 3:1). ${ }^{1} \mathrm{H}$ NMR $(300 \mathrm{MHz}$, Chloroform- $d$ ) $\delta 2.03-1.78(\mathrm{~m}, 2 \mathrm{H})$, $1.58-1.43(\mathrm{~m}, 2 \mathrm{H}), 1.29(\mathrm{~s}, 2 \mathrm{H}), 1.25-1.10(\mathrm{~m}, 14 \mathrm{H}), 0.89(\mathrm{~s}, 6 \mathrm{H}), 0.86(\mathrm{~s}, 6 \mathrm{H})$. ${ }^{13} \mathrm{C}\left\{{ }^{1} \mathrm{H},{ }^{19} \mathrm{~F}\right\}$ NMR (126 MHz, Chloroform- $d$ ) $\delta$ 118.0, 117.7, 109.0, 82.9, 52.4, 44.4, $34.0,31.5,28.0,27.4,24.7,15.0$, carbon attached to boron atom not observed. ${ }^{19} \mathrm{~F}\left\{{ }^{1} \mathrm{H}\right\}$ NMR (470 MHz, Chloroform- $d$ ) $\delta-80.7(\mathrm{t}, J=9.6 \mathrm{~Hz}),-115.2--115.4(\mathrm{~m}),-127.9-$ $128.1(\mathrm{~m}) .{ }^{11} \mathrm{~B}$ NMR $\left(96 \mathrm{MHz}, \mathrm{CDCl}_{3}\right) \delta 34.3$. HRMS (ESI) $\mathrm{m} / \mathrm{z}:[\mathrm{M}+\mathrm{Na}]^{+}$calcd. for $\mathrm{C}_{19} \mathrm{H}_{32} \mathrm{O}_{2} \mathrm{BF}_{7} \mathrm{Na}^{+}$459.2279; found 459.2274. FTIR (neat): $v\left(\mathrm{~cm}^{-1}\right)$ 2959, 1481, 1390, 1371, 1354, 1306, 1261, 1220, 1168, 1145, 1113, 1046, 967, 948, 909, 867, 846, 751, 724, 692, 668, 578 .

4,4,5,5-Tetramethyl-2-(8,8,9,9,10,10,11,11,12,12,13,13,13-tridecafluoro-2,4,4trimethyltridecan-2-yl)-1,3,2-dioxaborolane (9d)

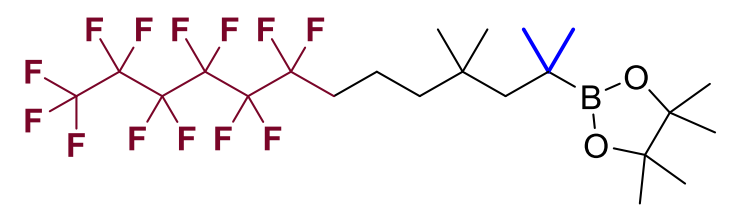

Following GP1 using 2-(2,2-dimethylpent-4-en-1-yl)-4,4,5,5-tetramethyl-1,3,2dioxaborolane (7a, $44.8 \mathrm{mg}, 0.20 \mathrm{mmol})$, isopropyl lithium $(0.31 \mathrm{~mL}, 0.22 \mathrm{mmol}, 0.7$ $\mathrm{M}$ in pentane) and perfluorohexyl bromide (119.7 $\mathrm{mg}, 0.3 \mathrm{mmol})$, flash chromatography eluting with pentane/DCM (3:1) afforded 9d as colorless sticky oil (79.9 mg, 68\% yield). $\mathrm{R}_{\mathrm{f}} 0.7$ (pentane/DCM = 3:1). ${ }^{1} \mathrm{H}$ NMR $(300 \mathrm{MHz}$, Chloroformd) $\delta 2.04-1.78(\mathrm{~m}, 2 \mathrm{H}), 1.61-1.44(\mathrm{~m}, 2 \mathrm{H}), 1.29(\mathrm{~s}, 2 \mathrm{H}), 1.25-1.10(\mathrm{~m}, 14 \mathrm{H}), 0.89$ $(\mathrm{s}, 6 \mathrm{H}), 0.86(\mathrm{~s}, 6 \mathrm{H}) .{ }^{13} \mathrm{C}\left\{{ }^{1} \mathrm{H},{ }^{19} \mathrm{~F}\right\}$ NMR $(126 \mathrm{MHz}$, Chloroform-d) $\delta$ 118.4, 117.3, 111.1, 110.3, 108.5, 83.0, 52.4, 44.4, 34.0, 31.8, 28.0, 27.4, 24.7, 15.1, carbon attached to boron atom not observed. ${ }^{19} \mathrm{~F}\left\{{ }^{1} \mathrm{H}\right\}$ NMR $(470 \mathrm{MHz}$, Chloroform- $d) \delta-80.9--81.0$ 
(m), -114.2--114.5 (m), -121.8--122.2 (m), -122.8--123.1 (m), -123.5--123.9 (m), -126.1 - -126.4 (m). ${ }^{11} \mathrm{~B}$ NMR $\left(96 \mathrm{MHz}, \mathrm{CDCl}_{3}\right) \delta 34.4$. HRMS (ESI) $\mathrm{m} / z:[\mathrm{M}+\mathrm{Na}]^{+}$ calcd. for $\mathrm{C}_{22} \mathrm{H}_{32} \mathrm{O}_{2} \mathrm{BF}_{13} \mathrm{Na}^{+}$609.2184; found 609.2186. FTIR (neat): v ( $\left.\mathrm{cm}^{-1}\right)$ 2979, 2939, 2874, 1481, 1372, 1307, 1237, 1189, 1143, 1072, 1022, 967, 846, 812, 729, 697, 652.

4,4,5,5-Tetramethyl-2-(8,8,9,9,10,10,11,11,12,12,13,13,13-tridecafluoro-4,4dimethyltridecan-2-yl)-1,3,2-dioxaborolane (9e)

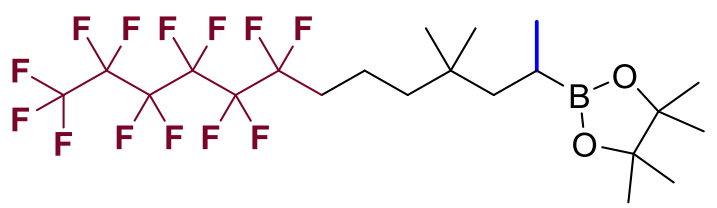

Following GP1 using 2-(2,2-dimethylpent-4-en-1-yl)-4,4,5,5-tetramethyl-1,3,2dioxaborolane (7a, $44.8 \mathrm{mg}, 0.20 \mathrm{mmol})$, ethyl lithium $(0.44 \mathrm{~mL}, 0.22 \mathrm{mmol}, 0.5 \mathrm{M}$ in cyclohexane/benzene) and perfluorohexyl bromide (119.7 $\mathrm{mg}, 0.3 \mathrm{mmol})$, flash chromatography eluting with pentane/DCM (3:1) afforded 9e as colorless sticky oil (78.1 mg, 68\% yield). $\mathrm{R}_{\mathrm{f}} 0.5$ (pentane/DCM = 3:1). ${ }^{1} \mathrm{H}$ NMR $(600 \mathrm{MHz}$, Chloroformd) $\delta 1.99(\mathrm{t}, J=7.8 \mathrm{~Hz}, 2 \mathrm{H}), 1.63-1.49(\mathrm{~m}, 3 \mathrm{H}), 1.27-1.19(\mathrm{~m}, 13 \mathrm{H}), 1.11-1.06(\mathrm{~m}$, 1H), $1.02-0.94(\mathrm{~m}, 4 \mathrm{H}), 0.85(\mathrm{~s}, 3 \mathrm{H}), 0.85(\mathrm{~s}, 3 \mathrm{H}) .{ }^{13} \mathrm{C}\left\{{ }^{1} \mathrm{H},{ }^{19} \mathrm{~F}\right\}$ NMR $(151 \mathrm{MHz}$, Chloroform- $d$ ) $\delta 118.4,117.2,111.1,110.3,108.5,82.8,46.0,41.9,33.5,31.8,27.1$, 27.0, 24.6, 24.6, 18.2, 15.0, carbon attached to boron atom not observed. ${ }^{19} \mathrm{~F}\left\{{ }^{1} \mathrm{H}\right\} \mathrm{NMR}$ (564 MHz, Chloroform- $d$ ) $\delta$-80.9 - -81.0 (m), -114.3 - -114.4 (m), -121.9- -122.1 (m), $-122.9--123.1(\mathrm{~m}),-123.6--123.9(\mathrm{~m}),-126.2--126.4(\mathrm{~m}) .{ }^{11} \mathrm{~B}$ NMR $(96 \mathrm{MHz}$, $\left.\mathrm{CDCl}_{3}\right) \delta$ 33.8. HRMS (ESI) $\mathrm{m} / z$ : $[\mathrm{M}+\mathrm{Na}]^{+}$calcd. for $\mathrm{C}_{21} \mathrm{H}_{30} \mathrm{O}_{2} \mathrm{BF}_{13} \mathrm{Na}^{+}$595.2027; found 595.2030. FTIR (neat): $v\left(\mathrm{~cm}^{-1}\right)$ 2979, 2960, 2938, 1465, 1381, 1367, 1316, 1237 , $1195,1143,1120,1073,1022,969,863,845,812,729,698,652$.

4,4,5,5-Tetramethyl-2-(9,9,10,10,11,11,12,12,13,13,14,14,14-tridecafluoro-3,5,5trimethyltetradecan-3-yl)-1,3,2-dioxaborolane (9f)

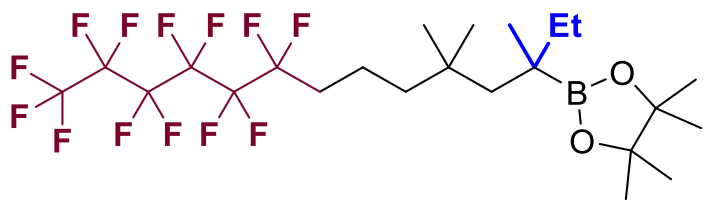

Following GP1 using 2-(2,2-dimethylpent-4-en-1-yl)-4,4,5,5-tetramethyl-1,3,2dioxaborolane (7a, $44.8 \mathrm{mg}, 0.20 \mathrm{mmol})$, isobutyl lithium $(0.16 \mathrm{~mL}, 0.22 \mathrm{mmol}, 1.4 \mathrm{M}$ in hexane) and perfluorohexyl bromide (119.7 $\mathrm{mg}, 0.3 \mathrm{mmol})$, flash chromatography eluting with pentane/DCM (4:1) afforded 9f as colorless sticky oil (70.7 mg, 59\% yield). $\mathrm{R}_{\mathrm{f}} 0.5$ (pentane/DCM = 4:1). ${ }^{1} \mathrm{H}$ NMR $(300 \mathrm{MHz}$, Chloroform- $d$ ) $\delta 2.05-1.80(\mathrm{~m}, 2 \mathrm{H})$, $1.59-1.32(\mathrm{~m}, 4 \mathrm{H}), 1.26-1.05(\mathrm{~m}, 16 \mathrm{H}), 0.89$ (s, 3H), $0.88(\mathrm{~s}, 3 \mathrm{H}), 0.86(\mathrm{~s}, 3 \mathrm{H}), 0.78$ $(\mathrm{t}, J=7.5 \mathrm{~Hz}, 3 \mathrm{H}) .{ }^{13} \mathrm{C}\left\{{ }^{1} \mathrm{H},{ }^{19} \mathrm{~F}\right\}$ NMR $(126 \mathrm{MHz}$, Chloroform- $d$ ) $\delta 118.4,117.2,111.1$, 110.3, 108.5, 83.0, 50.4, 44.6, 34.2, 34.0, 31.8, 28.3, 27.9, 25.2, 24.9, 21.9, 15.0, 9.2, carbon attached to boron atom not observed. ${ }^{19} \mathrm{~F}\left\{{ }^{1} \mathrm{H}\right\}$ NMR $(470 \mathrm{MHz}$, Chloroform- $d)$ $\delta-80.9--81.0(\mathrm{~m}),-114.3--114.4(\mathrm{~m}),-121.9--122.1(\mathrm{~m}),-122.8--123.1(\mathrm{~m}),-$ $123.6--123.9(\mathrm{~m}),-126.1--126.3(\mathrm{~m}) .{ }^{11} \mathrm{~B}$ NMR $\left(96 \mathrm{MHz}, \mathrm{CDCl}_{3}\right) \delta 34.0$. HRMS 
(ESI) $m / z:[\mathrm{M}+\mathrm{Na}]^{+}$calcd. for $\mathrm{C}_{23} \mathrm{H}_{34} \mathrm{O}_{2} \mathrm{BF}_{13} \mathrm{Na}^{+}$623.2341; found 623.2344. FTIR (neat): $v\left(\mathrm{~cm}^{-1}\right) 2966,1465,1371,1304,1237,1199,1143,1073,1025,967,908,843$, $735,697,651$.

2-(4,4-Diethyl-8,8,8-trifluoro-2-methyloctan-2-yl)-4,4,5,5-tetramethyl-1,3,2dioxaborolane $(\mathbf{9 g})$

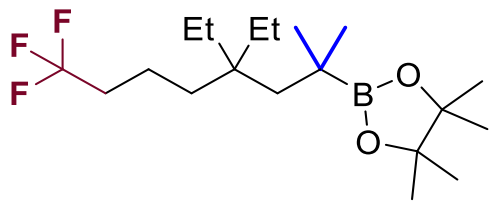

Following GP1 using 2-(2,2-diethylpent-4-en-1-yl)-4,4,5,5-tetramethyl-1,3,2dioxaborolane (7g, $50.4 \mathrm{mg}, 0.20 \mathrm{mmol})$, isopropyl lithium $(0.31 \mathrm{~mL}, 0.22 \mathrm{mmol}, 0.7$ $\mathrm{M}$ in pentane) and trifluoromethyl iodide $(0.3 \mathrm{~mL}, 0.3 \mathrm{M}, 1.0 \mathrm{M}$ in acetonitrile), flash chromatography eluting with pentane/DCM (3:1) afforded 9g as colorless sticky oil (42.7 mg, 59\% yield). $\mathrm{R}_{\mathrm{f}} 0.6$ (pentane/DCM = 4:1).

$1 \mathrm{mmol}$ scale reaction: Following GP1 using 2-(2,2-diethylpent-4-en-1-yl)-4,4,5,5tetramethyl-1,3,2- dioxaborolane $(7 \mathrm{~g}, 252 \mathrm{mg}, 1.00 \mathrm{mmol})$, isopropyl lithium $(1.5 \mathrm{~mL}$, $1.1 \mathrm{mmol}, 0.7 \mathrm{M}$ in pentane) and trifluoromethyl iodide (1.5, 1.5 m.mol, $1.0 \mathrm{M}$ in acetonitrile), flash chromatography eluting with pentane/DCM (3:1) afforded $\mathbf{9 g}$ as colorless sticky oil (145.6 mg, 40\% yield). Rf 0.6 (pentane/DCM = 4:1).

${ }^{1} \mathrm{H}$ NMR (300 MHz, Chloroform-d) $\delta 2.04-1.83(\mathrm{~m}, 2 \mathrm{H}), 1.48-1.35(\mathrm{~m}, 2 \mathrm{H}), 1.26$ (s, 2H), $1.25-1.17(\mathrm{~m}, 6 \mathrm{H}), 1.15(\mathrm{~s}, 12 \mathrm{H}), 0.90(\mathrm{~s}, 6 \mathrm{H}), 0.71(\mathrm{t}, J=7.4 \mathrm{~Hz}, 6 \mathrm{H}) .{ }^{13} \mathrm{C}\left\{{ }^{1} \mathrm{H}\right.$, $\left.{ }^{19} \mathrm{~F}\right\}$ NMR $\left(126 \mathrm{MHz}, \mathrm{CDCl}_{3}\right) \delta 127.2,82.9,45.2,38.6,36.2,34.7,29.4,27.6,24.8$, 16.2, 8.0, 7.9, carbon attached to boron atom not observed. ${ }^{19} \mathrm{~F}\left\{{ }^{1} \mathrm{H}\right\} \mathrm{NMR}(282 \mathrm{MHz}$, $\left.\mathrm{CDCl}_{3}\right) \delta$-66.3. ${ }^{11} \mathrm{~B} \mathrm{NMR}\left(96 \mathrm{MHz}, \mathrm{CDCl}_{3}\right) \delta 34.2$. HRMS (ESI) $\mathrm{m} / z:[\mathrm{M}+\mathrm{Na}]^{+}$calcd. for $\mathrm{C}_{19} \mathrm{H}_{36} \mathrm{O}_{2} \mathrm{BF}_{3} \mathrm{Na}^{+}$387.2656; found 387.2652. FTIR (neat): $v\left(\mathrm{~cm}^{-1}\right) 2971,2938$, 1462, 1371, 1305, 1252, 1229, 1167, 1137, 1083, 1008, 967, 908, 858, 690, 652.

4,4,5,5-Tetramethyl-2-(8,8,8-trifluoro-2-methyl-4,4-dipropyloctan-2-yl)-1,3,2dioxaborolane $(\mathbf{9 h})$

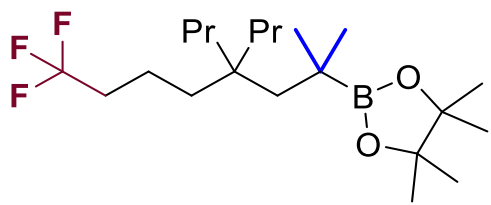

Following GP1 using 2-(2,2-dipropylpent-4-en-1-yl)-4,4,5,5-tetramethyl-1,3,2dioxaborolane (7h, $56.0 \mathrm{mg}, 0.20 \mathrm{mmol})$, isopropyl lithium $(0.31 \mathrm{~mL}, 0.22 \mathrm{mmol}, 0.7$ $\mathrm{M}$ in pentane) and trifluoromethyl iodide $(0.3 \mathrm{~mL}, 0.3 \mathrm{M}, 1.0 \mathrm{M}$ in acetonitrile), flash chromatography eluting with pentane/DCM (3:1) afforded $\mathbf{9 h}$ as colorless sticky oil (50.9 mg, 65\% yield). $\mathrm{R}_{\mathrm{f}} 0.7$ (pentane/DCM = 3:1). ${ }^{1} \mathrm{H}$ NMR (300 MHz, Chloroformd) $\delta 2.19-1.98(\mathrm{~m}, 2 \mathrm{H}), 1.67-1.52(\mathrm{~m}, 2 \mathrm{H}), 1.45(\mathrm{~s}, 2 \mathrm{H}), 1.40-1.27(\mathrm{~m}, J=4.0 \mathrm{~Hz}$, $22 \mathrm{H}), 1.06(\mathrm{~s}, 6 \mathrm{H}), 1.01-0.92(\mathrm{~m}, 6 \mathrm{H}) .{ }^{13} \mathrm{C}\left\{{ }^{1} \mathrm{H},{ }^{19} \mathrm{~F}\right\} \mathrm{NMR}\left(126 \mathrm{MHz}, \mathrm{CDCl}_{3}\right) \delta 127.2$, 82.9, 45.8, 40.4, 38.9, 37.4, 34.7, 27.5, 24.8, 16.8, 16.4, 15.0, carbon attached to boron atom not observed. ${ }^{19} \mathrm{~F}\left\{{ }^{1} \mathrm{H}\right\} \mathrm{NMR}\left(282 \mathrm{MHz}, \mathrm{CDCl}_{3}\right) \delta-66.3 .{ }^{11} \mathrm{~B} \mathrm{NMR}(96 \mathrm{MHz}$, $\left.\mathrm{CDCl}_{3}\right) \delta$ 33.8. HRMS (ESI) $\mathrm{m} / z:[\mathrm{M}+\mathrm{Na}]^{+}$calcd. for $\mathrm{C}_{21} \mathrm{H}_{40} \mathrm{O}_{2} \mathrm{BF}_{3} \mathrm{Na}^{+}$415.2970, 
found 415.2963. FTIR (neat): $v\left(\mathrm{~cm}^{-1}\right)$ 2957, 2934, 2873, 1459, 1390, 1371, 1304, 1252 , 1169, 1138, 1032, 967, 857, 745, 692, 654.

4,4,5,5-Tetramethyl-2-(2-methyl-1-(1-(4,4,4-trifluorobutyl)cyclopentyl)propan-2-yl)1,3,2-dioxaborolane (9i)

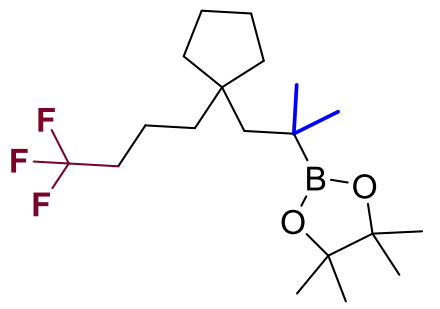

Following GP1 using 2-((1-allylcyclopentyl)methyl)-4,4,5,5-tetramethyl-1,3,2dioxaborolane (7i, $50.0 \mathrm{mg}, 0.20 \mathrm{mmol})$, isopropyl lithium $(0.31 \mathrm{~mL}, 0.22 \mathrm{mmol}, 0.7$ $\mathrm{M}$ in pentane) and trifluoromethyl iodide $(0.3 \mathrm{~mL}, 0.3 \mathrm{M}, 1.0 \mathrm{M}$ in acetonitrile), flash chromatography eluting with pentane/DCM (3:1) afforded 9i as colorless sticky oil (45.8 mg, 63\% yield). $\mathrm{R}_{\mathrm{f}} 0.5$ (pentane/DCM = 3:1). ${ }^{1} \mathrm{H}$ NMR (300 MHz, Chloroformd) $\delta 2.04-1.84(\mathrm{~m}, 2 \mathrm{H}), 1.60-1.42(\mathrm{~m}, 6 \mathrm{H}), 1.40(\mathrm{~s}, 2 \mathrm{H}), 1.38-1.21(\mathrm{~m}, 6 \mathrm{H}), 1.15$ (s, 12H), $0.89(\mathrm{~s}, 6 \mathrm{H}) .{ }^{13} \mathrm{C}\left\{{ }^{1} \mathrm{H},{ }^{19} \mathrm{~F}\right\} \mathrm{NMR}\left(151 \mathrm{MHz}, \mathrm{CDCl}_{3}\right) \delta 127.2,82.9,49.3,45.5$, $39.4,38.0,34.7,27.0,24.7,24.7,17.4$, carbon attached to boron atom not observed. ${ }^{19} \mathrm{~F}\left\{{ }^{1} \mathrm{H}\right\} \mathrm{NMR}\left(282 \mathrm{MHz}, \mathrm{CDCl}_{3}\right) \delta-66.4 .{ }^{11} \mathrm{~B} \mathrm{NMR}\left(96 \mathrm{MHz}, \mathrm{CDCl}_{3}\right) \delta 34.4$. HRMS (ESI) $m / z:[\mathrm{M}+\mathrm{Na}]^{+}$calcd. for $\mathrm{C}_{19} \mathrm{H}_{34} \mathrm{O}_{2} \mathrm{BF}_{3} \mathrm{Na}^{+}$385.2500; found 385.2495. FTIR (neat): $v\left(\mathrm{~cm}^{-1}\right)$ 2954, 2869, 1478, 1390, 1371, 1305, 1252, 1214, 1139, 1056, 967, 849, 692.

4,4,5,5-Tetramethyl-2-(2-methyl-1-(1-(4,4,4-trifluorobutyl)cyclohexyl)propan-2-yl)1,3,2-dioxaborolane (9j)

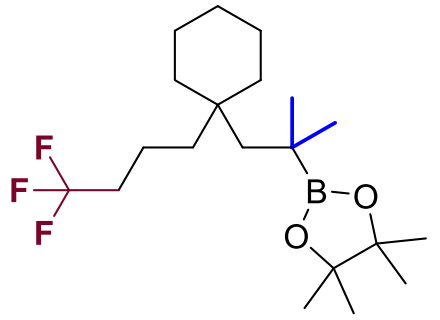

Following GP1 using 2-((1-allylcyclohexyl)methyl)-4,4,5,5-tetramethyl-1,3,2dioxaborolane (7j, $52.8 \mathrm{mg}, 0.20 \mathrm{mmol})$, isopropyl lithium $(0.31 \mathrm{~mL}, 0.22 \mathrm{mmol}, 0.7$ $\mathrm{M}$ in pentane) and trifluoromethyl iodide $(0.3 \mathrm{~mL}, 0.3 \mathrm{M}, 1.0 \mathrm{M}$ in acetonitrile), flash chromatography eluting with pentane/DCM (3:1) afforded 9j as colorless sticky oil (46.1 mg, 62\% yield). $\mathrm{R}_{\mathrm{f}} 0.5$ (pentane/DCM = 3:1). ${ }^{1} \mathrm{H}$ NMR $(300 \mathrm{MHz}$, Chloroformd) $\delta 2.06-1.83(\mathrm{~m}, 2 \mathrm{H}), 1.51-1.08(\mathrm{~m}, 16 \mathrm{H}), 1.15(\mathrm{~s}, 12 \mathrm{H}), 0.90(\mathrm{~s}, 6 \mathrm{H}) .{ }^{13} \mathrm{C}\left\{{ }^{1} \mathrm{H},{ }^{19} \mathrm{~F}\right\}$ NMR $\left(151 \mathrm{MHz}, \mathrm{CDCl}_{3}\right) \delta 127.3,82.9,50.3,36.0,35.6,35.5,34.7,27.8,26.1,24.7$, $21.7,15.8$, carbon attached to boron atom not observed. ${ }^{19} \mathrm{~F}\left\{{ }^{1} \mathrm{H}\right\}$ NMR $(282 \mathrm{MHz}$, $\left.\mathrm{CDCl}_{3}\right) \delta-66.3 .{ }^{11} \mathrm{~B} \mathrm{NMR}\left(96 \mathrm{MHz}, \mathrm{CDCl}_{3}\right) \delta 34.3$. HRMS (ESI) $m / z:[\mathrm{M}+\mathrm{Na}]^{+}$calcd. for $\mathrm{C}_{20} \mathrm{H}_{36} \mathrm{O}_{2} \mathrm{BF}_{3} \mathrm{Na}^{+}$399.2656; found 399.2660. FTIR (neat): $v\left(\mathrm{~cm}^{-1}\right)$ 2976, 2929, 2861, 1479, 1457, 1371, 1352, 1303, 1252, 1226, 1196, 1142, 1133, 1055, 1001, 967 , $857,713,691,668,652$. 
4,4,5,5-Tetramethyl-2-(2-methyl-1-(4-(4,4,4-trifluorobutyl)tetrahydro-2H-pyran-4yl)propan-2-yl)-1,3,2-dioxaborolane (9k)

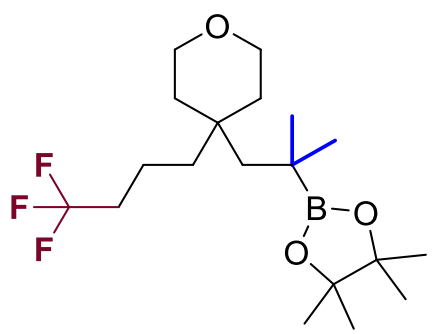

Following GP1 using 2-((4-allyltetrahydro-2H-pyran-4-yl)methyl)-4,4,5,5tetramethyl-1,3,2-dioxaborolane (7k, $53.2 \mathrm{mg}, 0.20 \mathrm{mmol})$, isopropyl lithium $(0.31 \mathrm{~mL}$, $0.22 \mathrm{mmol}, 0.7 \mathrm{M}$ in pentane) and trifluoromethyl iodide $(0.3 \mathrm{~mL}, 0.3 \mathrm{M}, 1.0 \mathrm{M}$ in acetonitrile), flash chromatography eluting with pentane/Et ${ }_{2} \mathrm{O}(3: 1)$ afforded $9 \mathbf{k}$ as colorless sticky oil (52.3 mg, 69\% yield). $\mathrm{R}_{\mathrm{f}} 0.4$ (pentane/ $\left.\mathrm{Et}_{2} \mathrm{O}=3: 1\right) .{ }^{1} \mathrm{H}$ NMR (300 $\mathrm{MHz}$, Chloroform- $d$ ) $\delta 3.73-3.42(\mathrm{~m}, 4 \mathrm{H}), 2.08-1.87(\mathrm{~m}, 2 \mathrm{H}), 1.64-1.50(\mathrm{~m}, 2 \mathrm{H})$, $1.49-1.42(\mathrm{~m}, 4 \mathrm{H}), 1.37(\mathrm{~s}, 2 \mathrm{H}), 1.33-1.23(\mathrm{~m}, 2 \mathrm{H}), 1.15(\mathrm{~s}, 12 \mathrm{H}), 0.92(\mathrm{~s}, 6 \mathrm{H})$. ${ }^{13} \mathrm{C}\left\{{ }^{1} \mathrm{H},{ }^{19} \mathrm{~F}\right\}$ NMR $\left(126 \mathrm{MHz}, \mathrm{CDCl}_{3}\right) \delta 127.1,83.0,63.6,48.7,35.8,35.7,34.4,34.0$, $27.7,24.7,15.8$, carbon attached to boron atom not observed. ${ }^{19} \mathrm{~F}\left\{{ }^{1} \mathrm{H}\right\} \mathrm{NMR}(282 \mathrm{MHz}$, $\left.\mathrm{CDCl}_{3}\right) \delta$-66.3. ${ }^{11} \mathrm{~B} \mathrm{NMR}\left(96 \mathrm{MHz}, \mathrm{CDCl}_{3}\right) \delta 33.7$. HRMS (ESI) $\mathrm{m} / z:[\mathrm{M}+\mathrm{Na}]^{+}$calcd. for $\mathrm{C}_{19} \mathrm{H}_{34} \mathrm{O}_{3} \mathrm{BF}_{3} \mathrm{Na}^{+}$401.2449; found 401.2446. FTIR (neat): $v\left(\mathrm{~cm}^{-1}\right)$ 2957, 2859, 1478, 1371, 1305, 1252, 1167, 1138, 1107, 1064, 1008, 967, 848, 691, 632.

4,4,5,5-Tetramethyl-2-(2-methyl-1-(1-(4,4,4-trifluorobutyl)cycloheptyl)propan-2-yl)1,3,2-dioxaborolane (9I)

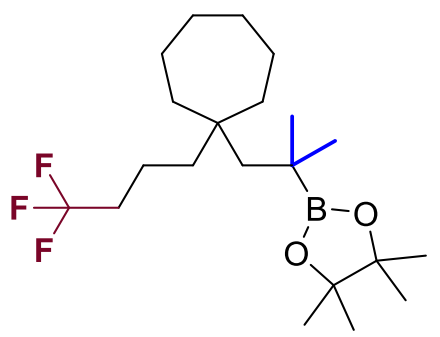

Following GP1 using 2-((1-allylcycloheptyl)methyl)-4,4,5,5-tetramethyl-1,3,2dioxaborolane (7l, $55.6 \mathrm{mg}, 0.20 \mathrm{mmol})$, isopropyl lithium $(0.31 \mathrm{~mL}, 0.22 \mathrm{mmol}, 0.7$ $\mathrm{M}$ in pentane) and trifluoromethyl iodide $(0.3 \mathrm{~mL}, 0.3 \mathrm{M}, 1.0 \mathrm{M}$ in acetonitrile), flash chromatography eluting with pentane/DCM (3:1) afforded 91 as colorless sticky oil (47.7 mg, 61\% yield). $\mathrm{R}_{\mathrm{f}} 0.5$ (pentane/ $\left.\mathrm{DCM}=3: 1\right)$. ${ }^{1} \mathrm{H}$ NMR $(300 \mathrm{MHz}$, Chloroformd) $\delta 2.05-1.81(\mathrm{~m}, 2 \mathrm{H}), 1.54-1.22(\mathrm{~m}, 18 \mathrm{H}), 1.16(\mathrm{~s}, 12 \mathrm{H}), 0.89(\mathrm{~s}, 6 \mathrm{H}) .{ }^{13} \mathrm{C}\left\{{ }^{1} \mathrm{H},{ }^{19} \mathrm{~F}\right\}$ NMR $\left(126 \mathrm{MHz}, \mathrm{CDCl}_{3}\right) \delta 127.3,82.9,50.1$, 39.6, 39.5, 39.4, 34.7, 30.8, 27.6, 24.8, $22.9,16.4$, carbon attached to boron atom not observed. ${ }^{19} \mathrm{~F}\left\{{ }^{1} \mathrm{H}\right\} \mathrm{NMR}(282 \mathrm{MHz}$, $\left.\mathrm{CDCl}_{3}\right) \delta-66.3 .{ }^{11} \mathrm{~B} \mathrm{NMR}\left(96 \mathrm{MHz}, \mathrm{CDCl}_{3}\right) \delta 34.3$. HRMS (ESI) $\mathrm{m} / z:[\mathrm{M}+\mathrm{Na}]^{+}$calcd. for $\mathrm{C}_{21} \mathrm{H}_{38} \mathrm{O}_{2} \mathrm{BF}_{3} \mathrm{Na}^{+}$413.2813; found 413.2806. FTIR (neat): $v\left(\mathrm{~cm}^{-1}\right)$ 2978, 2926, 2857, 1390, 1371, 1352, 1305, 1253, 1196, 1138, 1046, 1005, 967, 846, 688, 651. 
4,4,5,5-Tetramethyl-2-(8,8,8-trifluoro-2,4-dimethyl-4-phenyloctan-2-yl)-1,3,2dioxaborolane $(\mathbf{9 m})$<smiles>CC(C)(CCCC(F)(F)F)c1ccccc1</smiles>

Following GP1 using 4,4,5,5-tetramethyl-2-(2-methyl-2-phenylpent-4-en-1-yl)-1,3,2dioxaborolane (7m, $57.2 \mathrm{mg}, 0.20 \mathrm{mmol})$, isopropyl lithium $(0.31 \mathrm{~mL}, 0.22 \mathrm{mmol}, 0.7$ $\mathrm{M}$ in pentane) and trifluoromethyl iodide $(0.3 \mathrm{~mL}, 0.3 \mathrm{M}, 1.0 \mathrm{M}$ in acetonitrile), flash chromatography eluting with pentane/DCM (2:1) afforded $\mathbf{9 m}$ as colorless sticky oil (40.6 mg, 51\% yield). $\mathrm{R}_{\mathrm{f}} 0.4$ (pentane/ DCM = 2:1). ${ }^{1} \mathrm{H}$ NMR $(300 \mathrm{MHz}$, Chloroformd) $\delta 7.25-7.13(\mathrm{~m}, 4 \mathrm{H}), 7.12-7.00(\mathrm{~m}, 1 \mathrm{H}), 1.92-1.69(\mathrm{~m}, 4 \mathrm{H}), 1.68-1.56(\mathrm{~m}, 1 \mathrm{H})$, $1.52-1.34(\mathrm{~m}, 1 \mathrm{H}), 1.29(\mathrm{~s}, 3 \mathrm{H}), 1.16(\mathrm{~s}, 12 \mathrm{H}), 1.30-1.10(\mathrm{~m}, 1 \mathrm{H}) 0.98-0.83(\mathrm{~m}$, $1 \mathrm{H}), 0.79$ (s, 3H), 0.33 (s, 3H). ${ }^{13} \mathrm{C}\left\{{ }^{1} \mathrm{H},{ }^{19} \mathrm{~F}\right\} \mathrm{NMR}\left(126 \mathrm{MHz}, \mathrm{CDCl}_{3}\right) \delta 148.2,127.8$, 127.1, 126.4, 125.3, 83.0, 54.1, 45.2, 41.0, 34.2, 29.0, 24.8, 24.6, 22.4, 16.7, carbon attached to boron atom not observed. ${ }^{19} \mathrm{~F}\left\{{ }^{1} \mathrm{H}\right\} \mathrm{NMR}\left(282 \mathrm{MHz}, \mathrm{CDCl}_{3}\right) \delta$-66.2. ${ }^{11} \mathrm{~B}$ NMR $\left(160 \mathrm{MHz}, \mathrm{CDCl}_{3}\right) \quad \delta$ 34.1. HRMS (ESI) $\mathrm{m} / \mathrm{z}:[\mathrm{M}+\mathrm{Na}]^{+}$calcd. for $\mathrm{C}_{22} \mathrm{H}_{34} \mathrm{O}_{2} \mathrm{BF}_{3} \mathrm{Na}^{+}$421.2500; found 421.2495. FTIR (neat): $v\left(\mathrm{~cm}^{-1}\right)$ 2976, 2939, 1474, 1446, 1371, 1305, 1275, 1252, 1203, 1127, 1056, 1008, 967, 849, 769, 700.

4,4,5,5-Tetramethyl-2-(8,8,8-trifluoro-4-isopropyl-2-methyloctan-2-yl)-1,3,2dioxaborolane $(\mathbf{9 n})$<smiles>CC(C)C(CCCC(F)(F)F)CC(C)(C)B1OC(C)(C)C(C)(C)O1</smiles>

Following GP1 using 2-(2-isopropylpent-4-en-1-yl)-4,4,5,5-tetramethyl-1,3,2dioxaborolane (7n, $47.6 \mathrm{mg}, 0.20 \mathrm{mmol})$, isopropyl lithium $(0.31 \mathrm{~mL}, 0.22 \mathrm{mmol}, 0.7$ $\mathrm{M}$ in pentane) and trifluoromethyl iodide $(0.3 \mathrm{~mL}, 0.3 \mathrm{M}, 1.0 \mathrm{M}$ in acetonitrile $)$, flash chromatography eluting with pentane/DCM $(3: 1)$ afforded 9 n as colorless sticky oil (35.0 mg, 50\% yield). $\mathrm{R}_{\mathrm{f}} 0.5$ (pentane/ DCM = 3:1). ${ }^{1} \mathrm{H}$ NMR (300 MHz, Chloroformd) $\delta 2.07-1.85(\mathrm{~m}, 2 \mathrm{H}), 1.70-1.56(\mathrm{~m}, 1 \mathrm{H}), 1.55-1.35(\mathrm{~m}, 2 \mathrm{H}), 1.31-1.17(\mathrm{~m}, 3 \mathrm{H})$, $1.15(\mathrm{~s}, 6 \mathrm{H}), 1.15(\mathrm{~s}, 6 \mathrm{H}), 1.13-0.97(\mathrm{~m}, 3 \mathrm{H}), 0.84(\mathrm{~s}, 6 \mathrm{H}), 0.76(\mathrm{~s}, 3 \mathrm{H}), 0.73(\mathrm{~s}, 3 \mathrm{H})$. ${ }^{13} \mathrm{C} \mathrm{NMR}\left(126 \mathrm{MHz}, \mathrm{CDCl}_{3}\right) \delta 127.3,82.9,41.6,40.6,34.2,30.2,29.1,25.8,25.7,24.8$, $24.7,20.4,19.0,18.3$, carbon attached to boron atom not observed. ${ }^{19} \mathrm{~F}$ NMR (282 $\left.\mathrm{MHz}, \mathrm{CDCl}_{3}\right) \delta-66.4 .{ }^{11} \mathrm{~B} \mathrm{NMR}\left(160 \mathrm{MHz}, \mathrm{CDCl}_{3}\right) \delta 34.4$. HRMS (ESI) $\mathrm{m} / z:[\mathrm{M}+\mathrm{Na}]^{+}$ calcd. for $\mathrm{C}_{18} \mathrm{H}_{34} \mathrm{O}_{2} \mathrm{BF}_{3} \mathrm{Na}^{+}$373.2500; found 373.2496. FTIR (neat): $v\left(\mathrm{~cm}^{-1}\right) 2959$, 1476, 1390, 1372, 1306, 1259, 1141, 1107, 1016, 967, 852, 806, 691. 


\section{NMR Spectra}
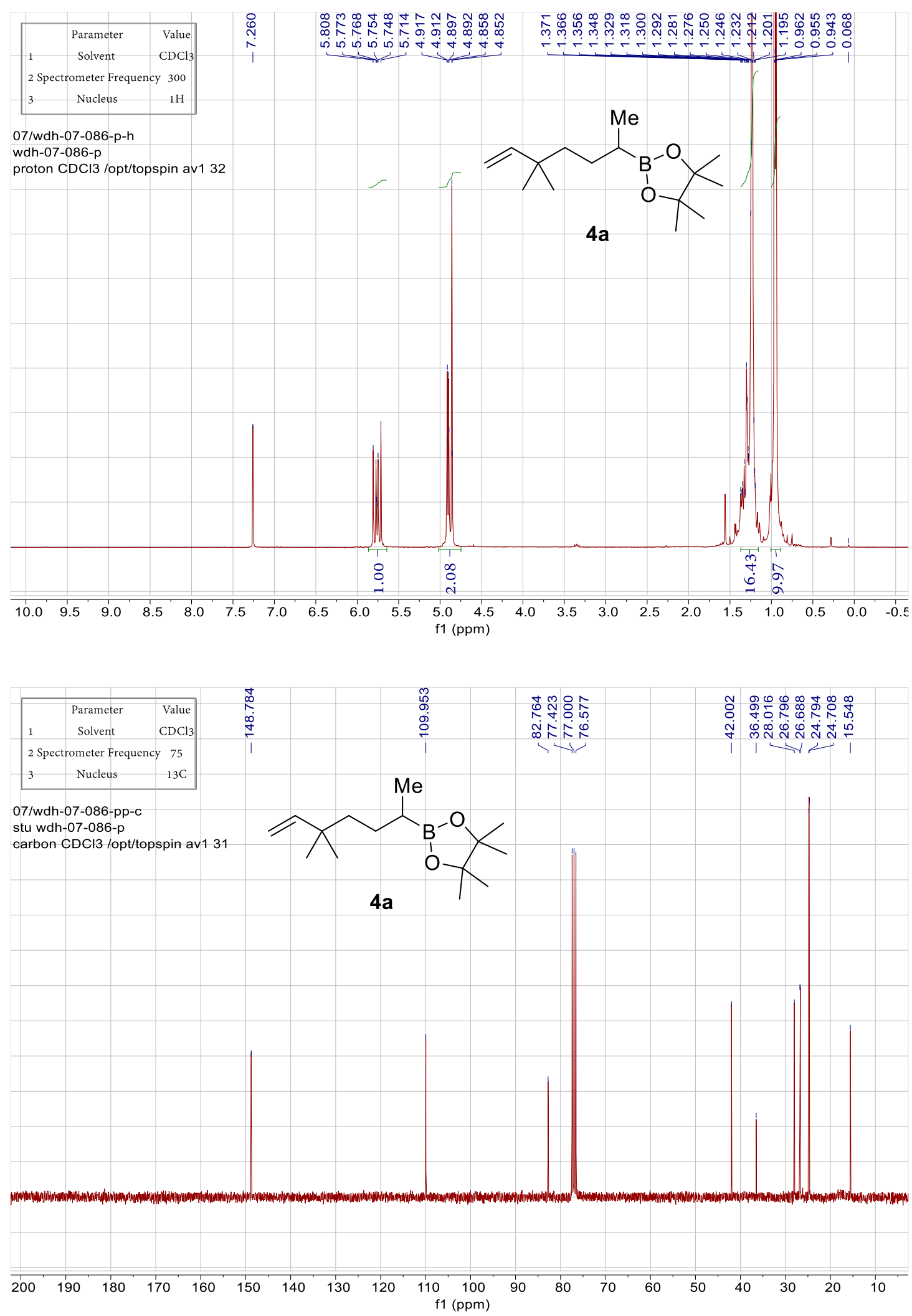

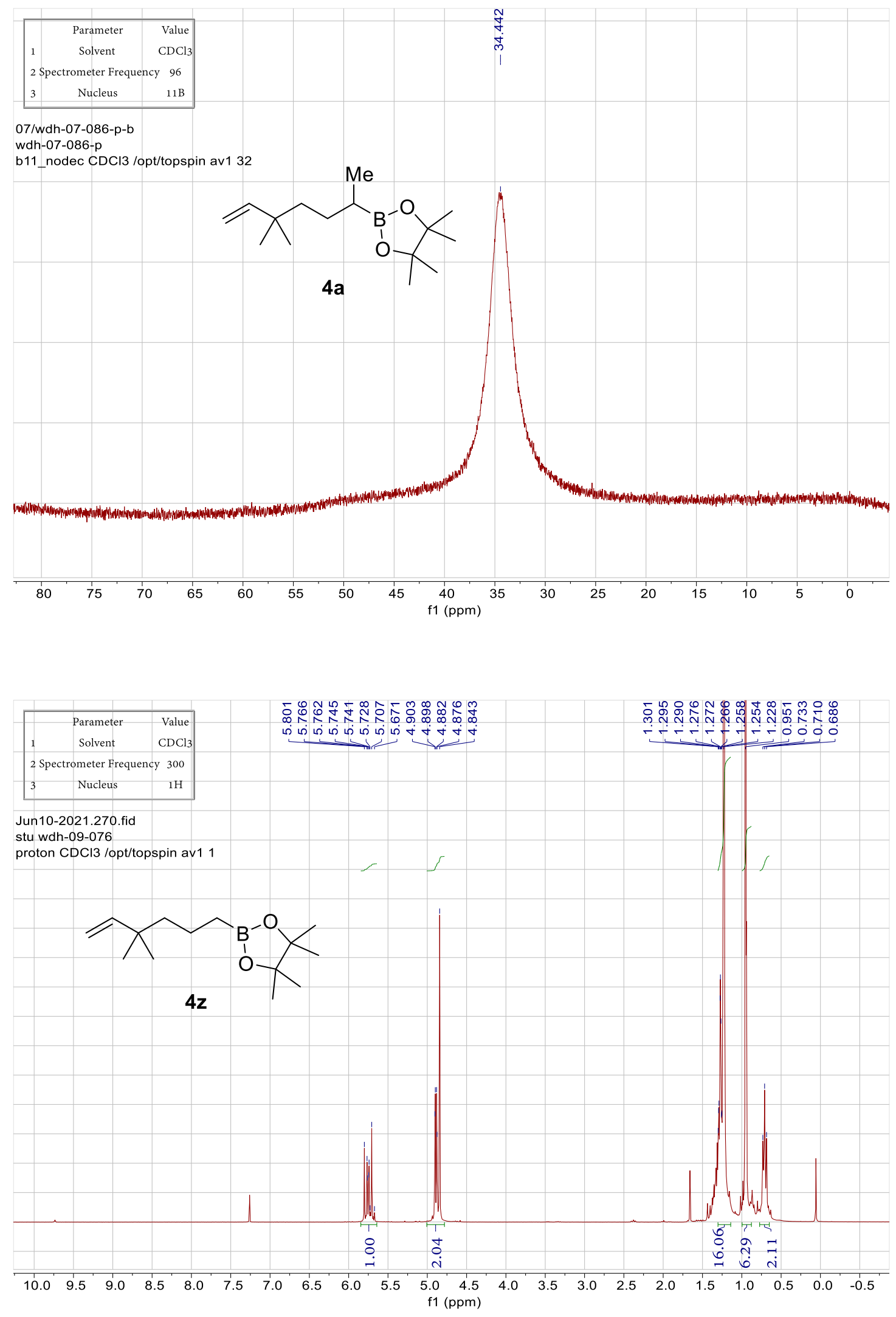


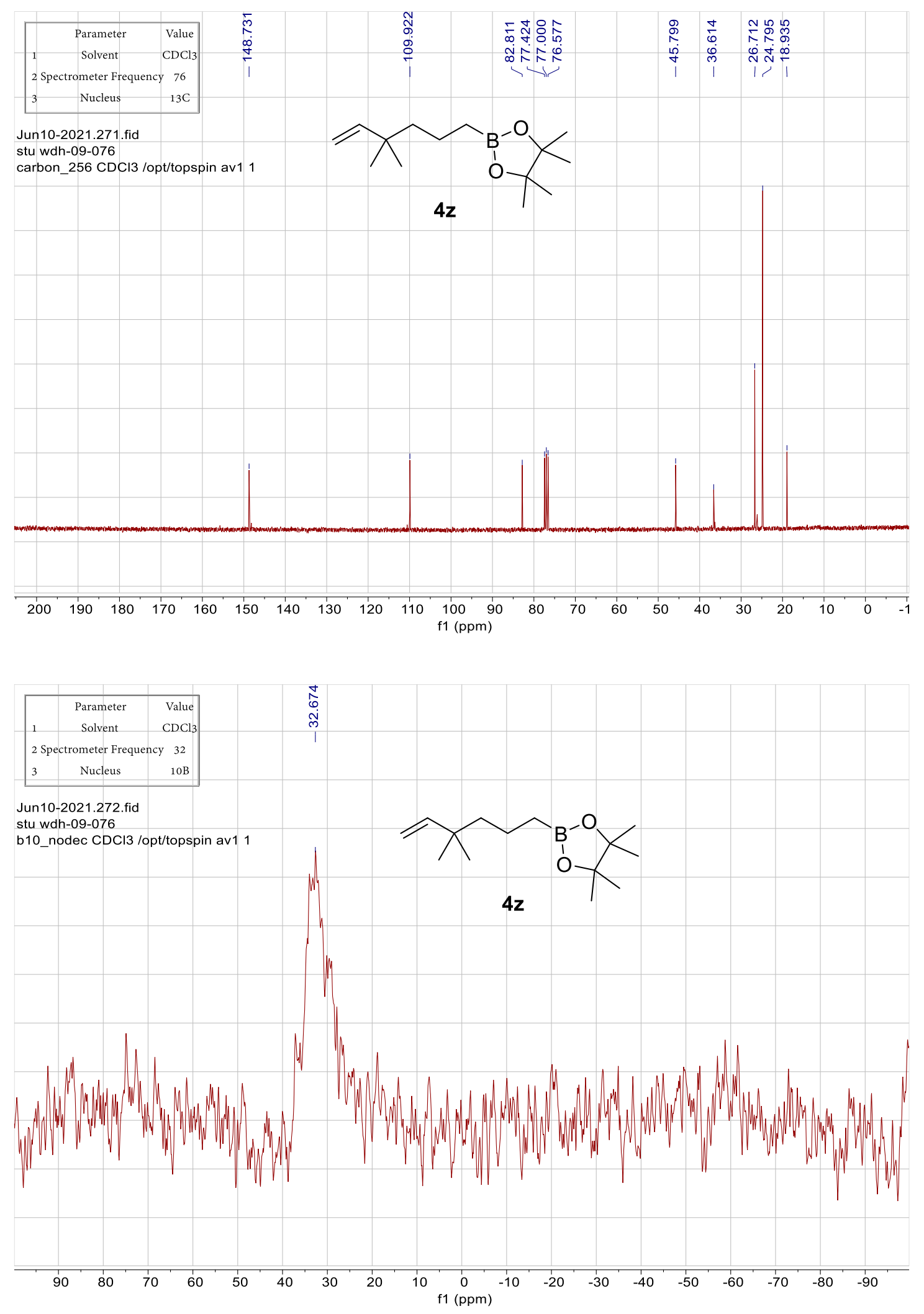



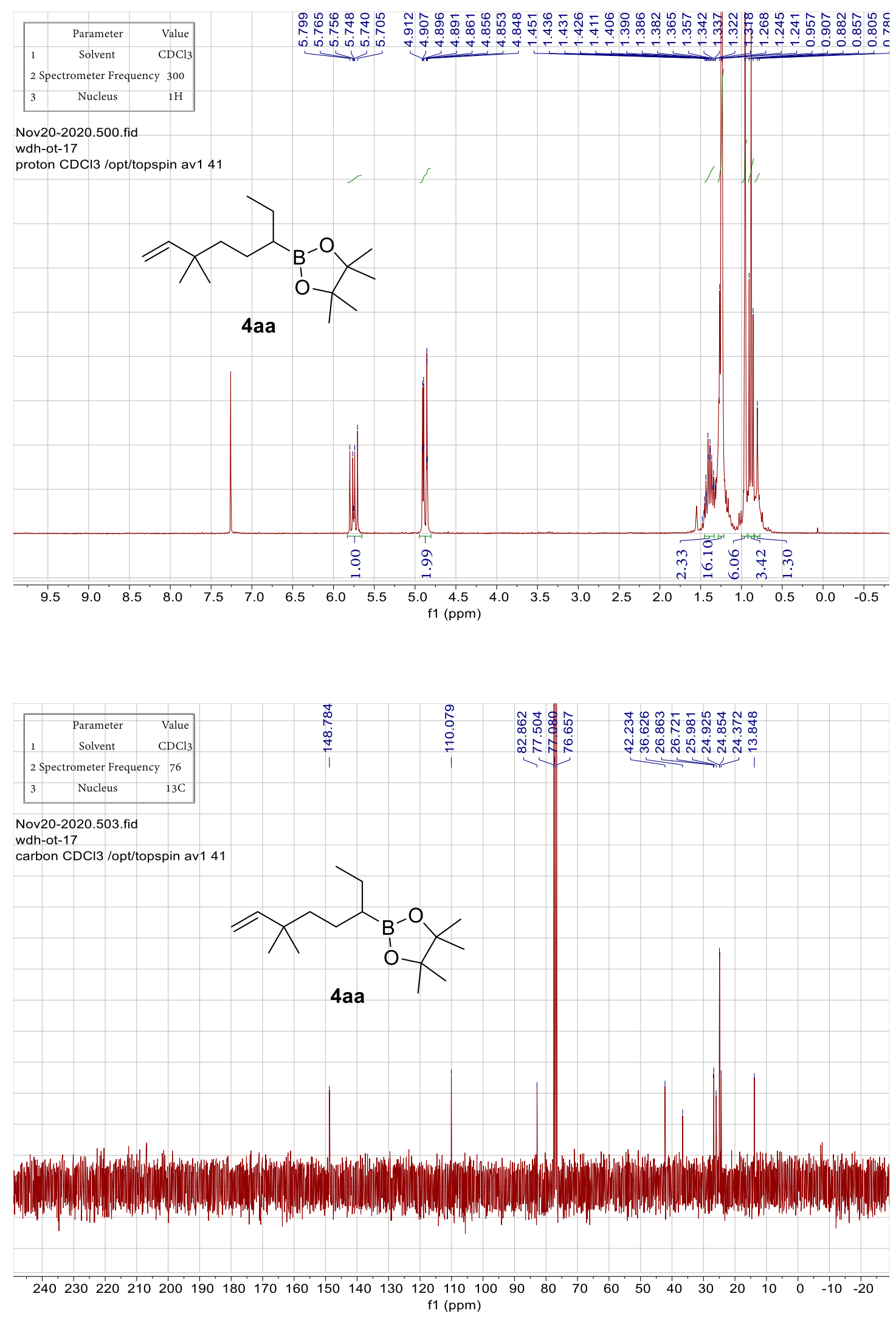

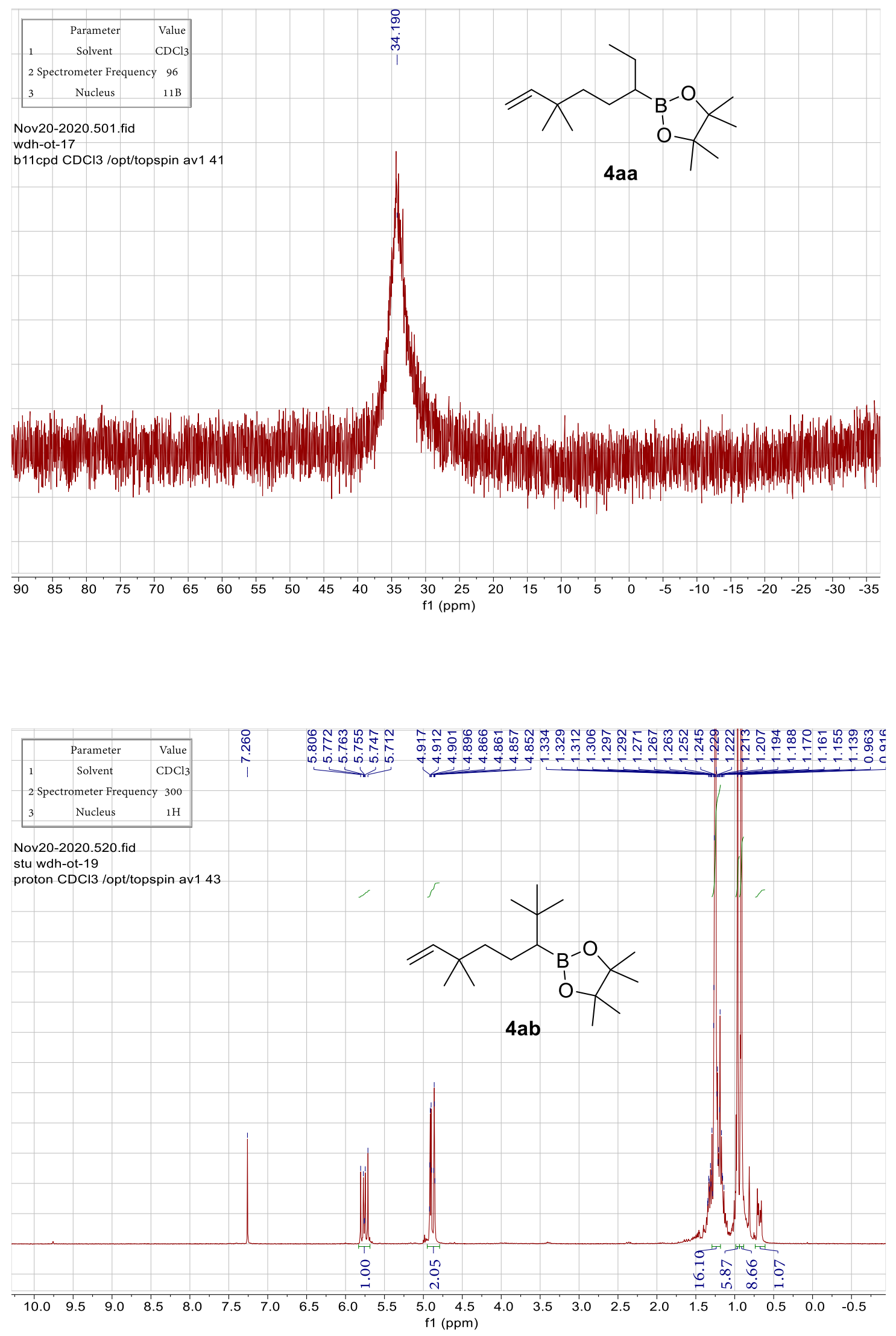

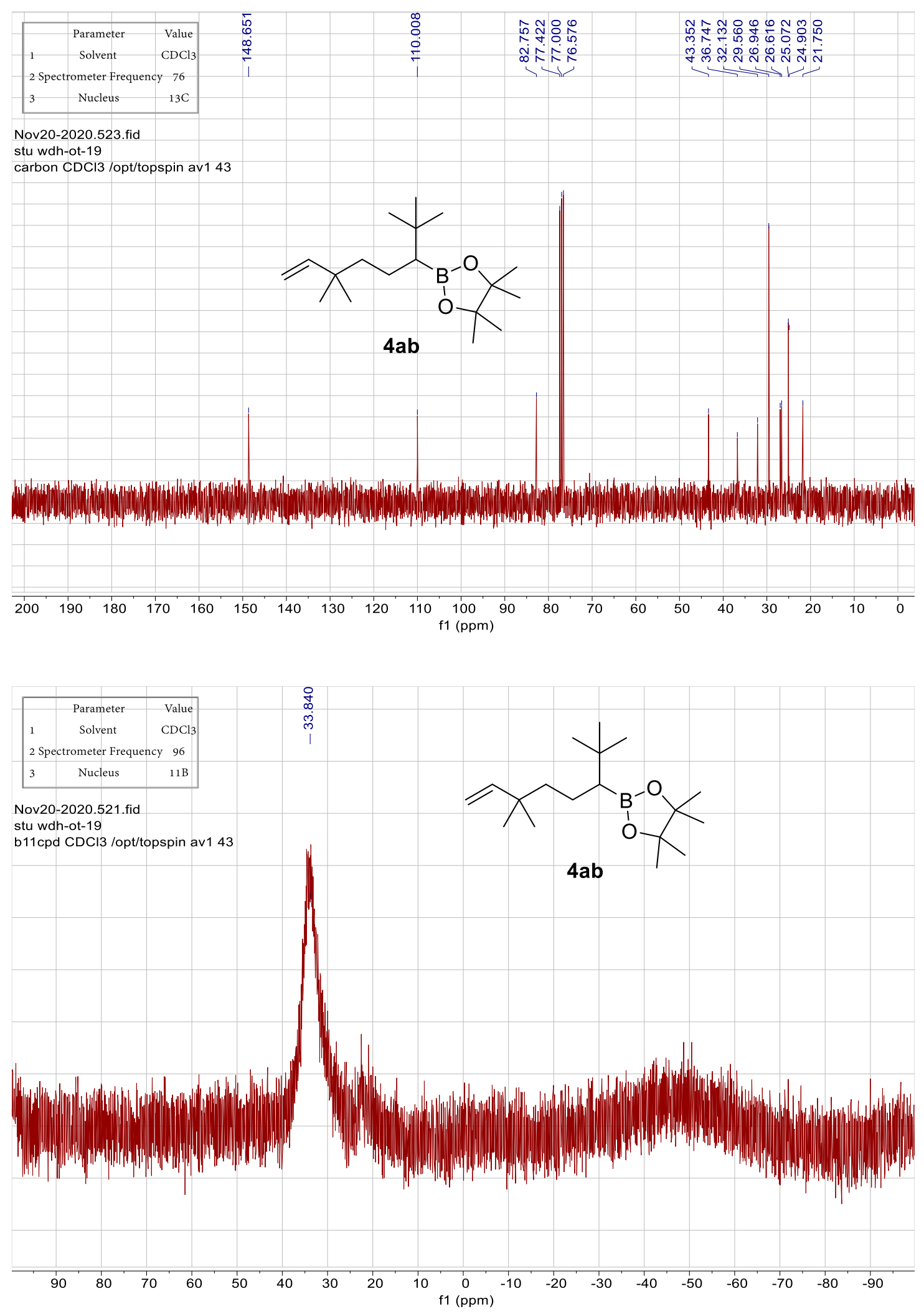

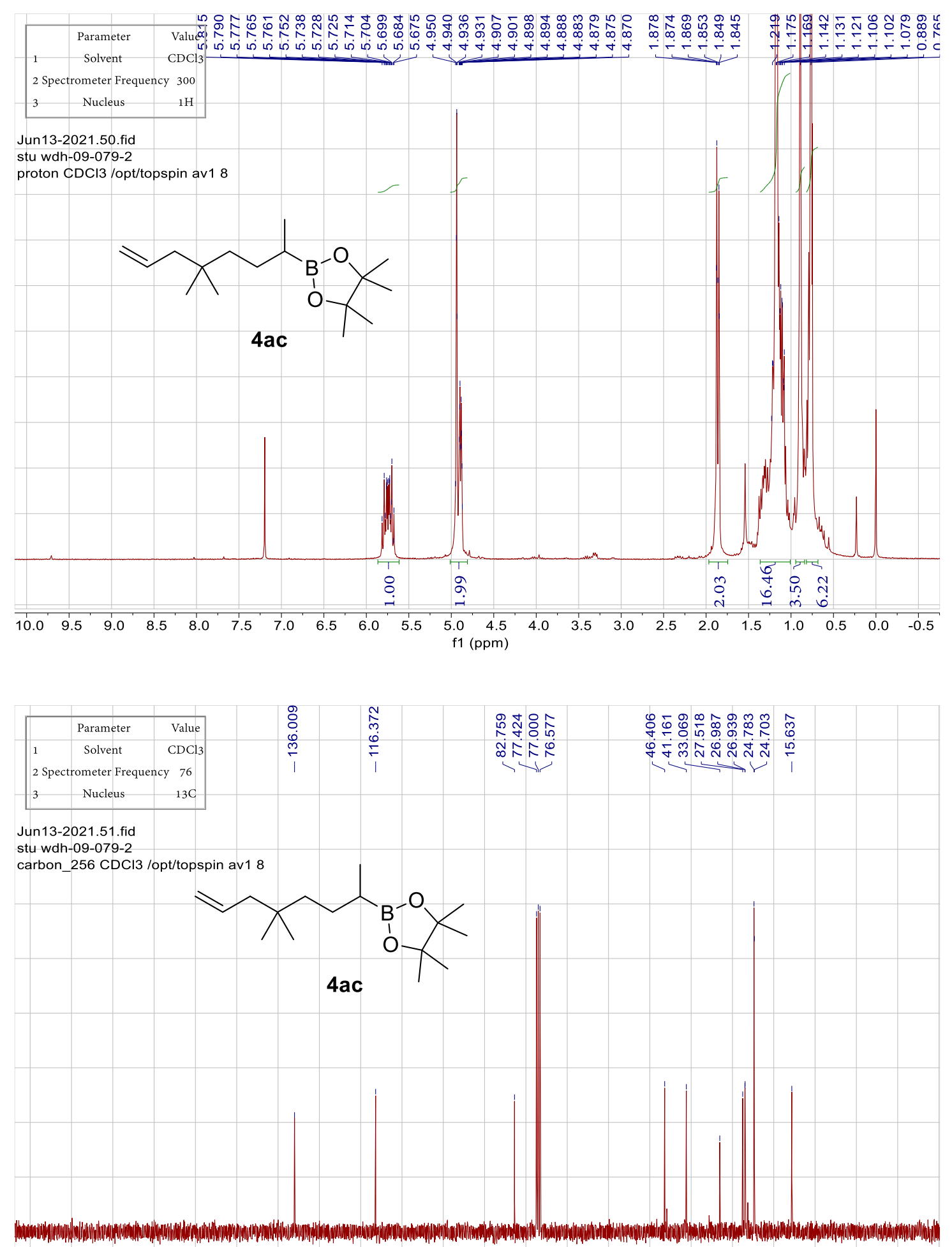

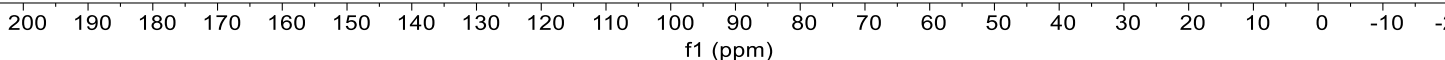



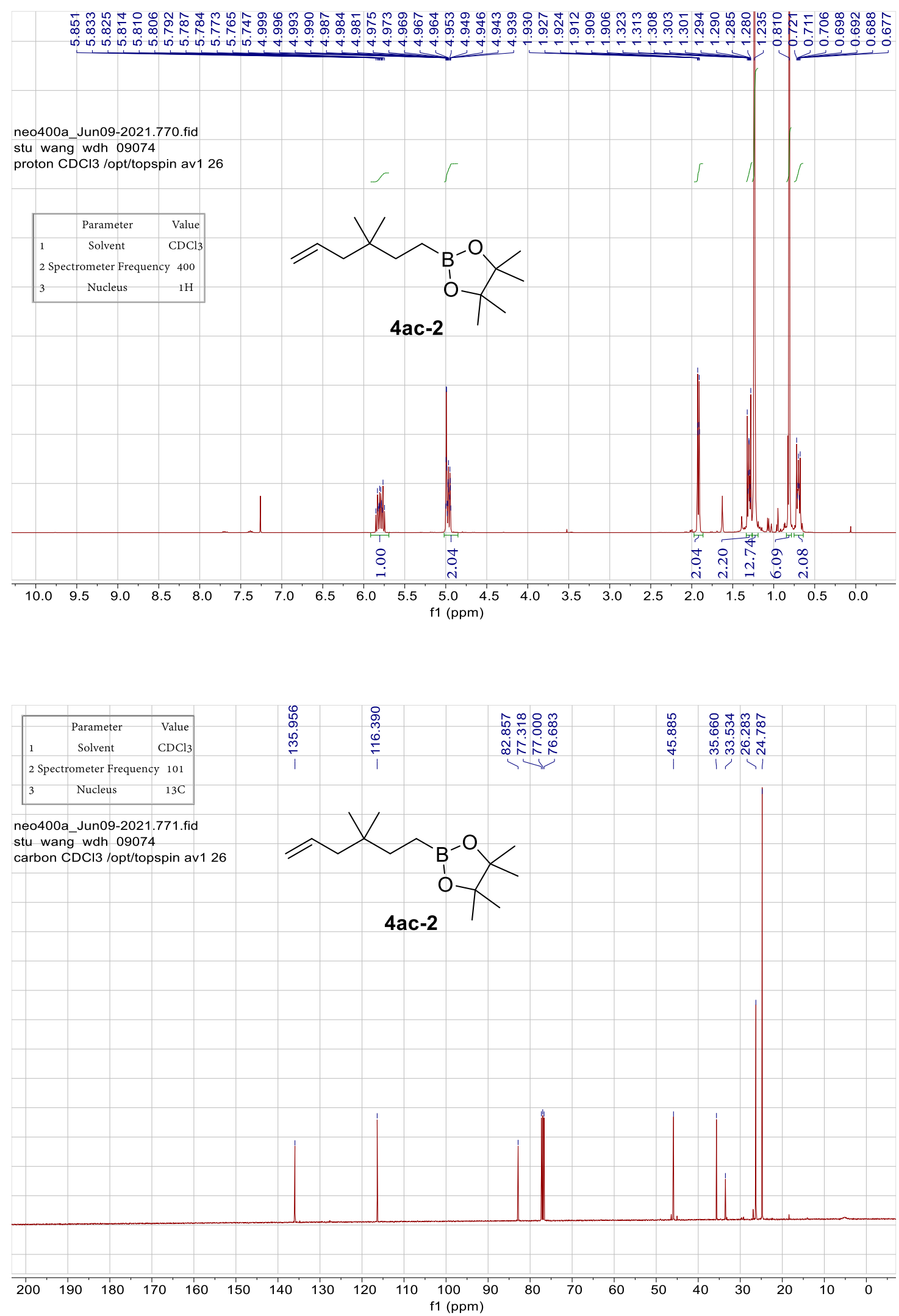

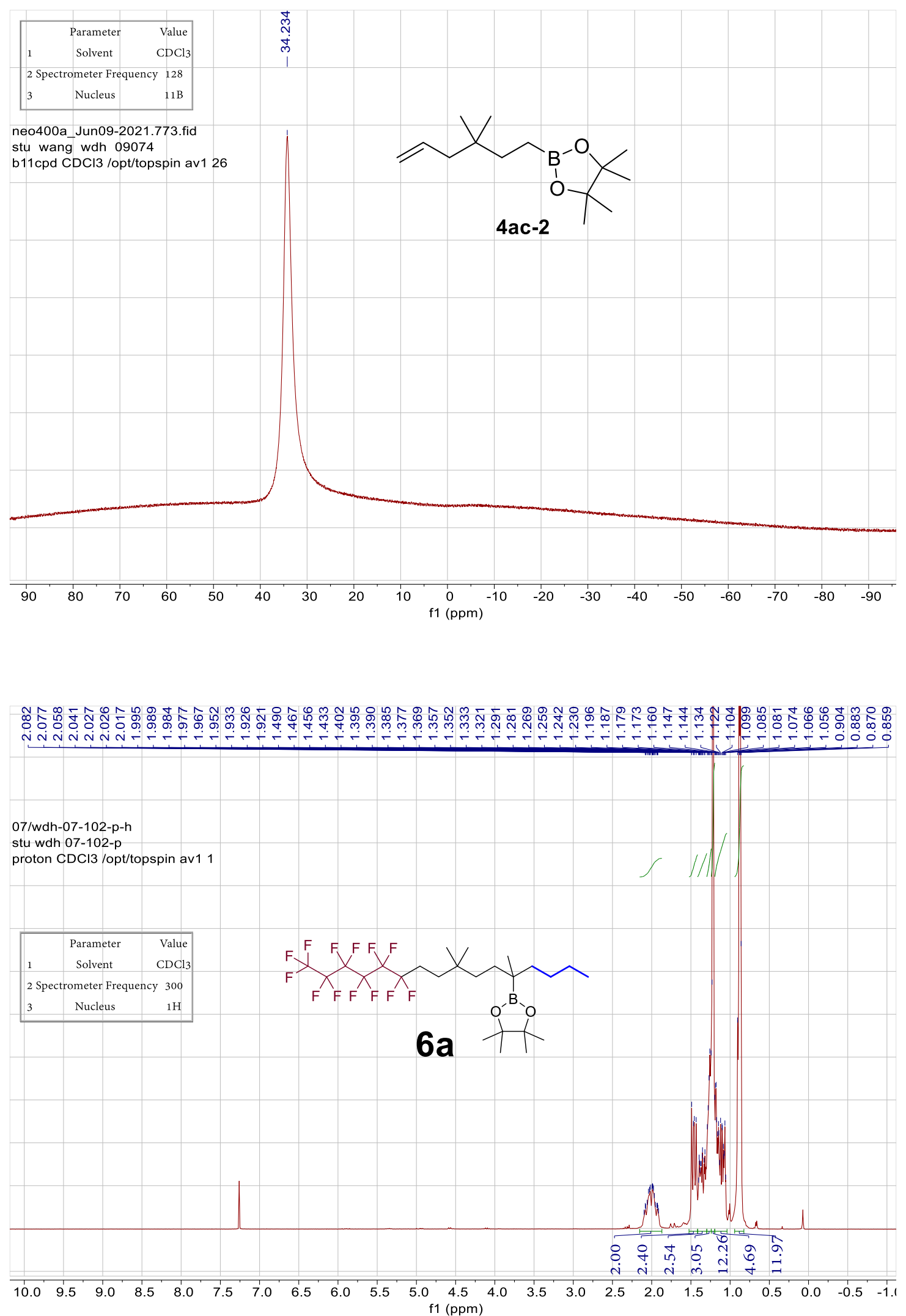

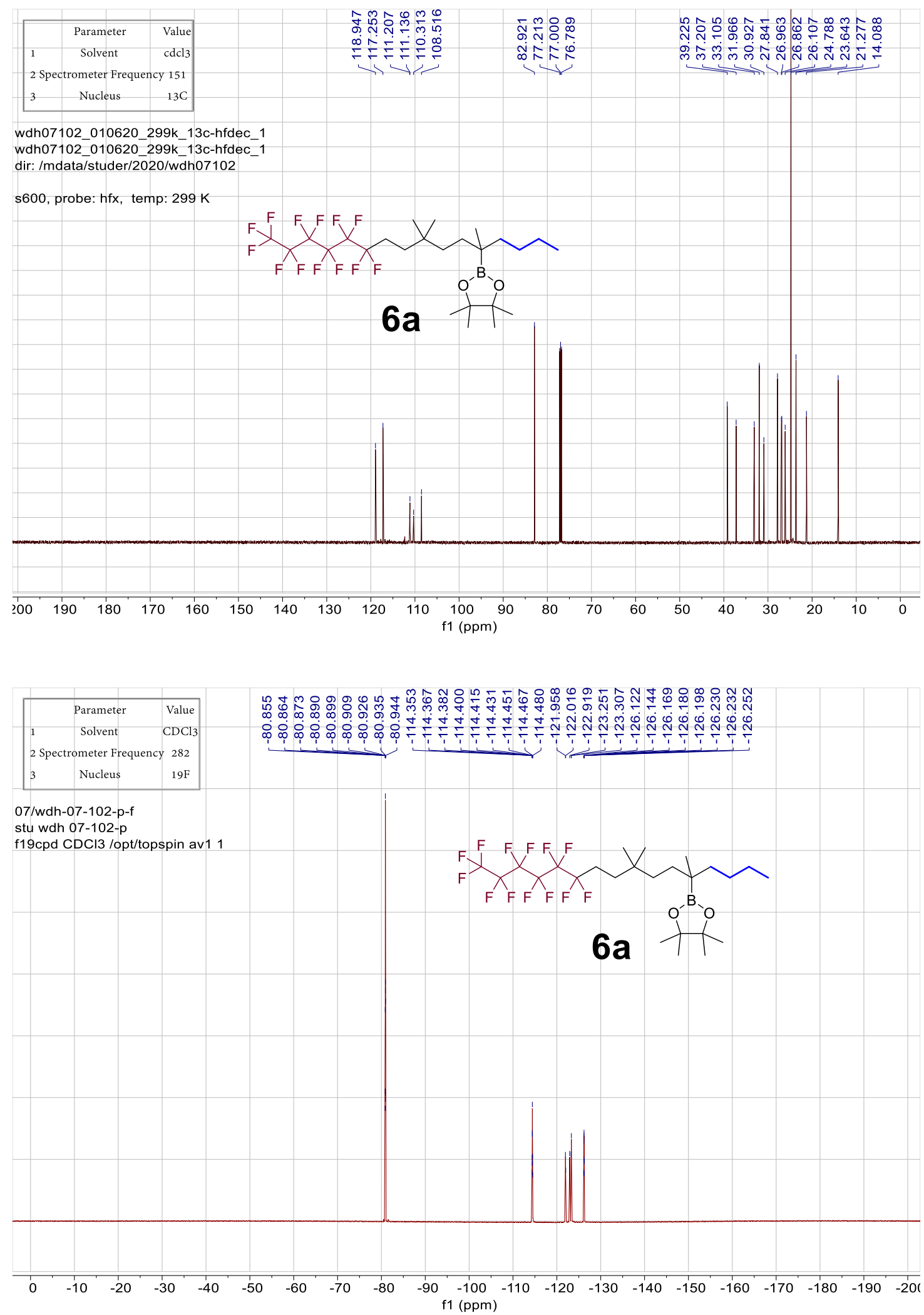

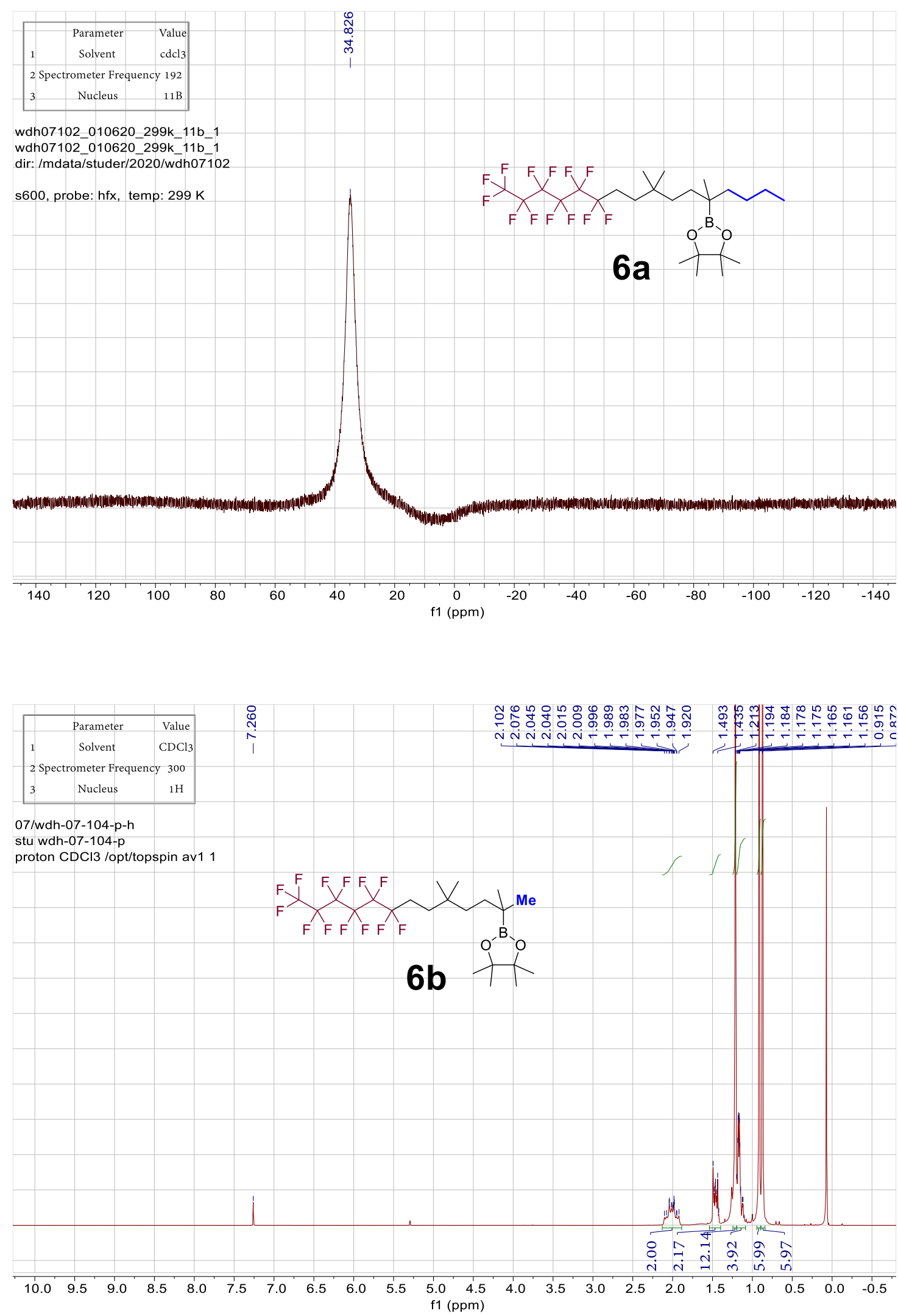


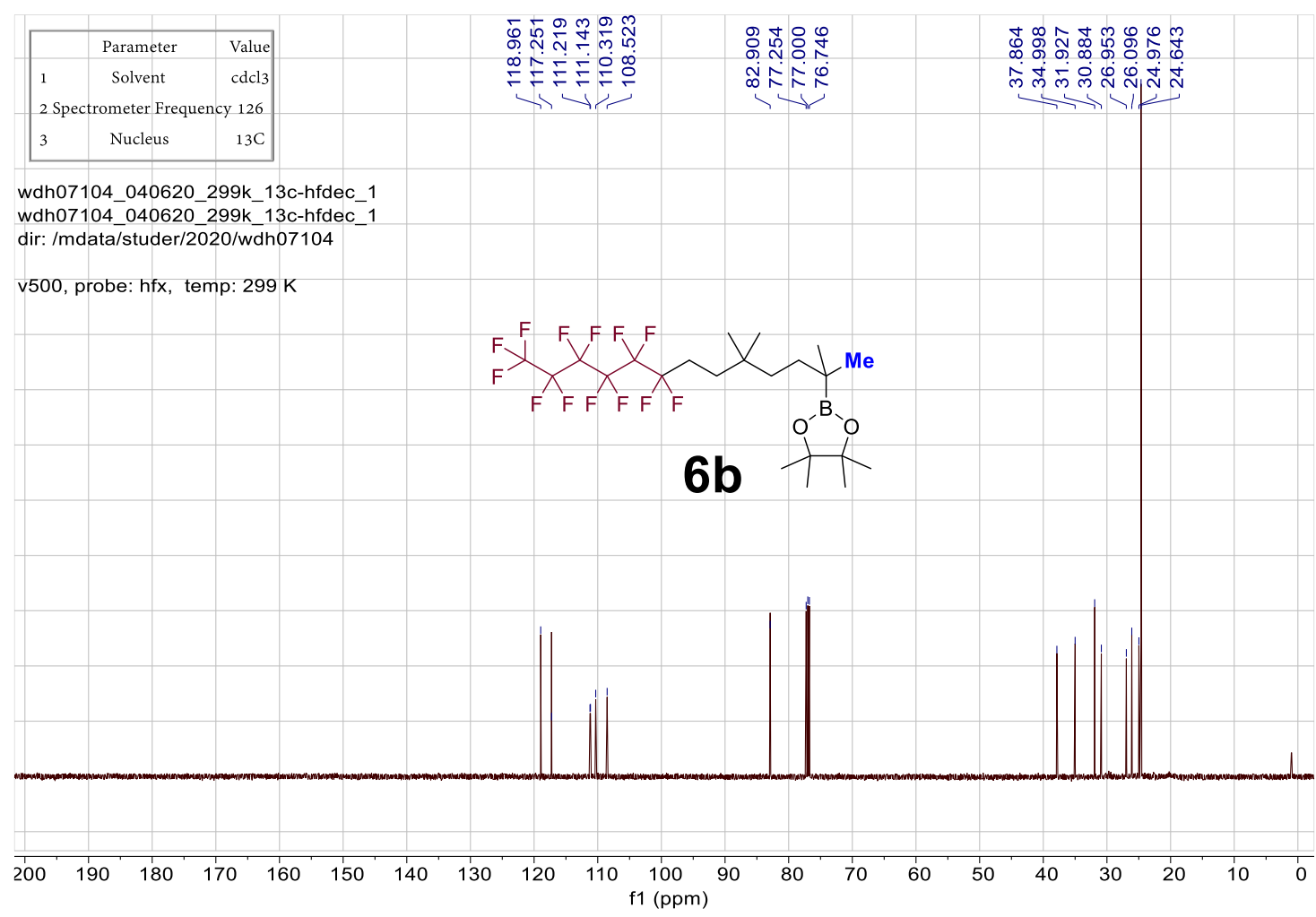

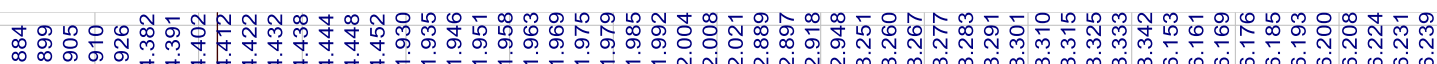

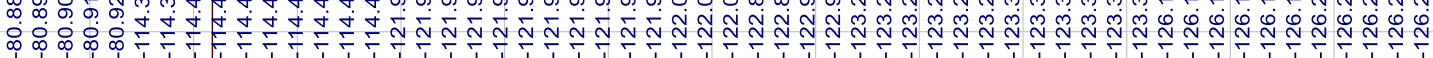

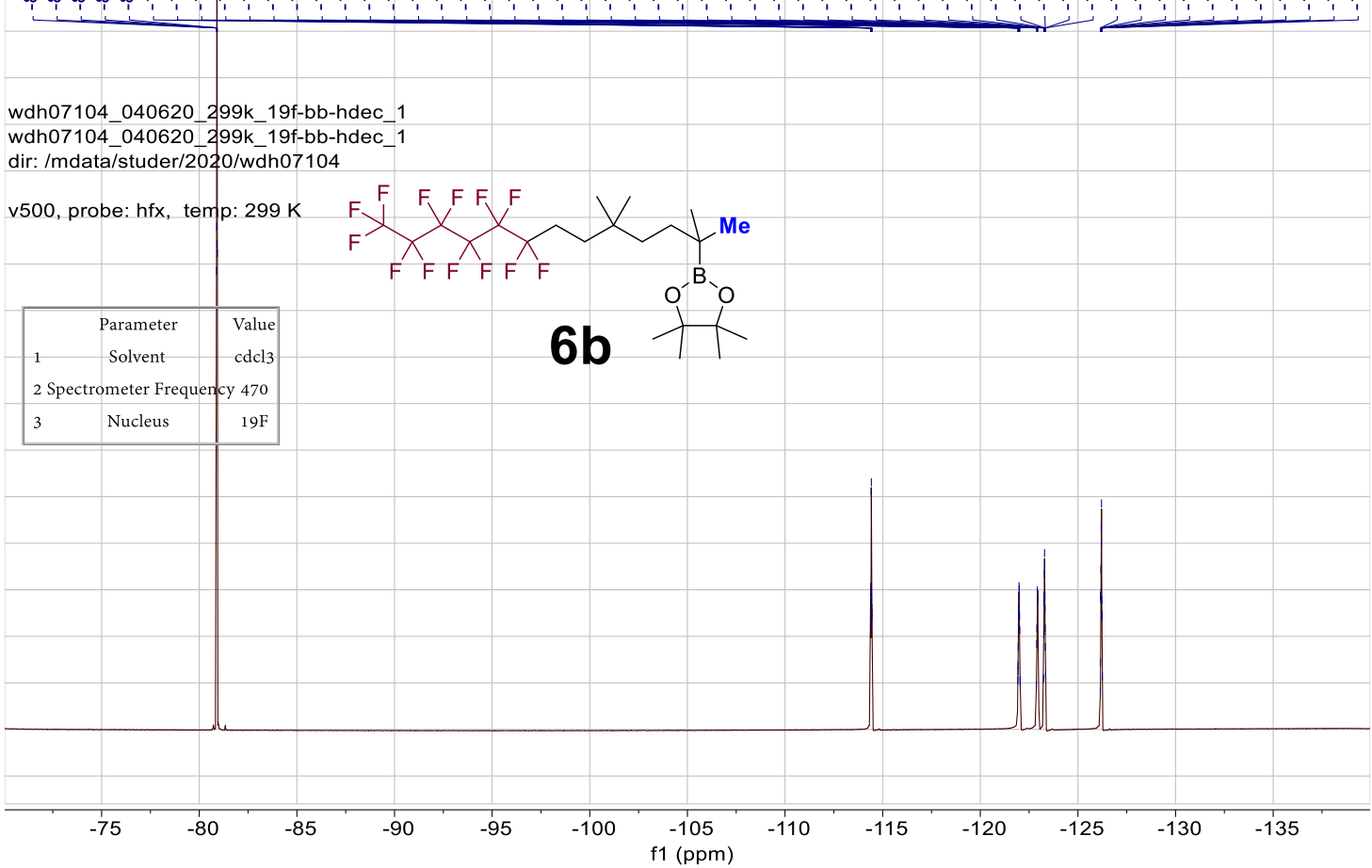



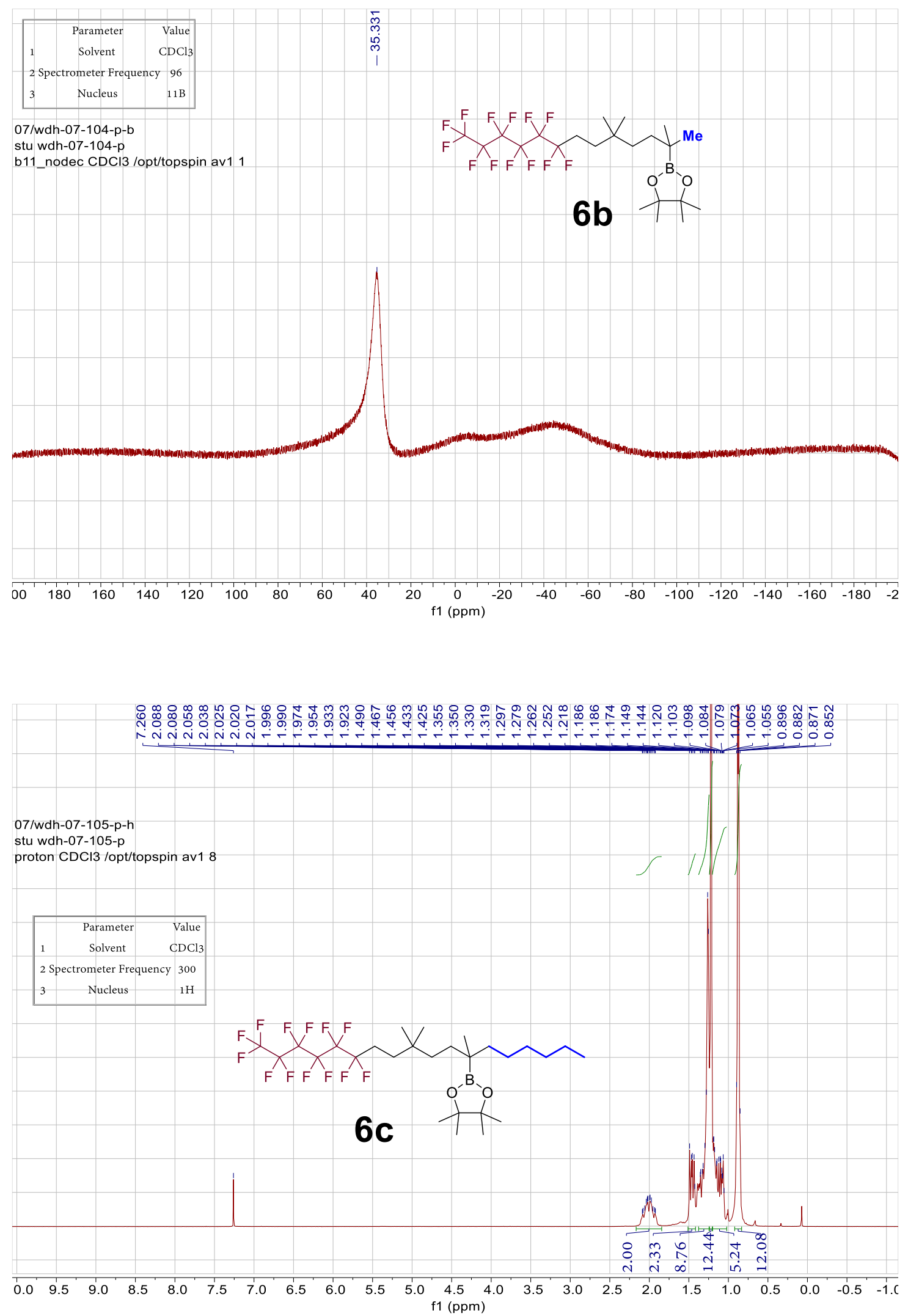


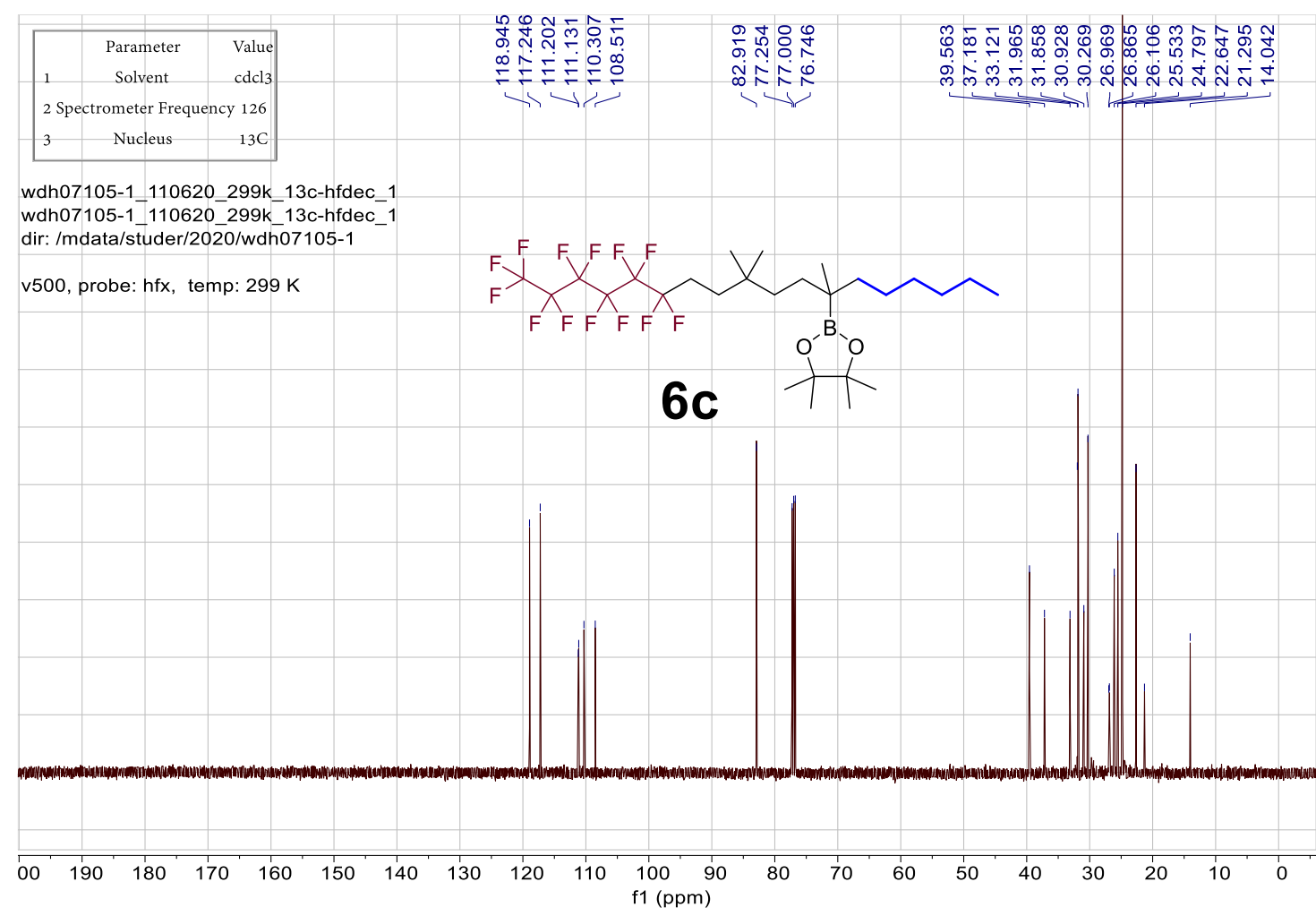

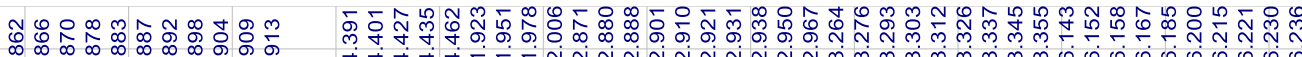

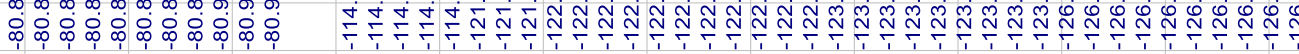

wdh07105-1 110620 299k 19f-bb-hdec 1

wdh07105-1_110620_299k_19f-bb-hdec

dir: /mdata/studer/2020/wdh07105-1

v500, probe: hfx, temp: $299 \mathrm{~K}$

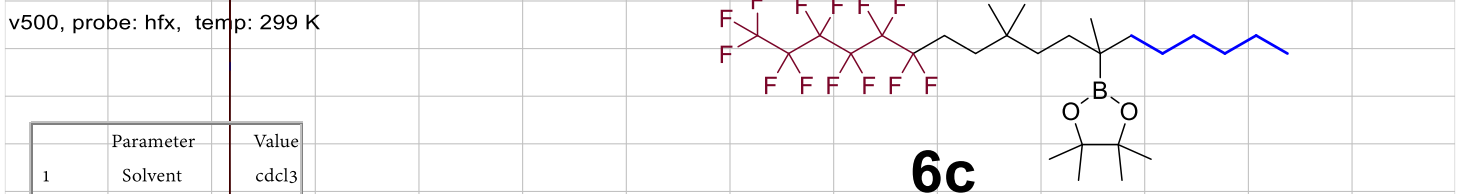

\begin{tabular}{l|l|l|l|l|l|l|}
1 & Solvent & $\operatorname{cdcl} 3$ & & 6
\end{tabular}

2 Spectrometer Frequepcy 470

\begin{tabular}{ll|l}
3 & Nucleus & $19 \mathrm{~F}$ \\
\hline
\end{tabular}

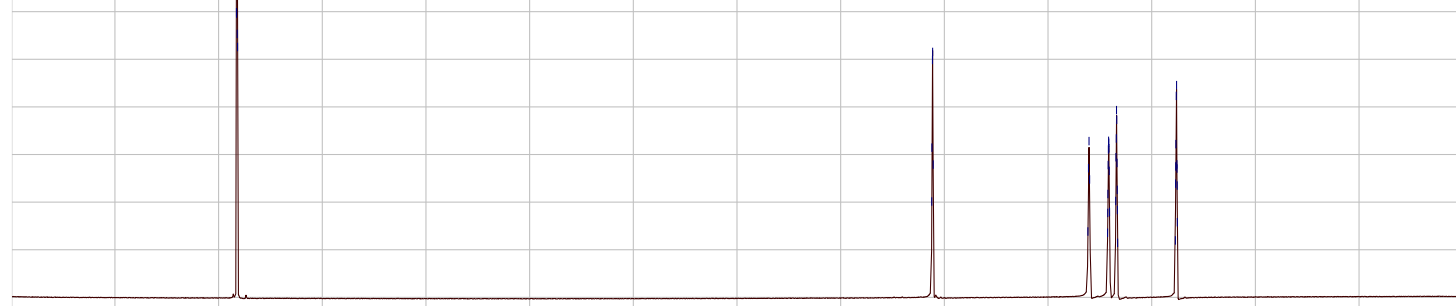

\begin{tabular}{|c|c|c|c|c|c|c|c|c|c|c|c|c|}
\hline-75 & -80 & -85 & -90 & -95 & -100 & $\begin{array}{c}-105 \\
\mathrm{f} 1(\mathrm{ppm})\end{array}$ & -110 & -115 & -120 & -125 & -130 & -135 \\
\hline
\end{tabular}



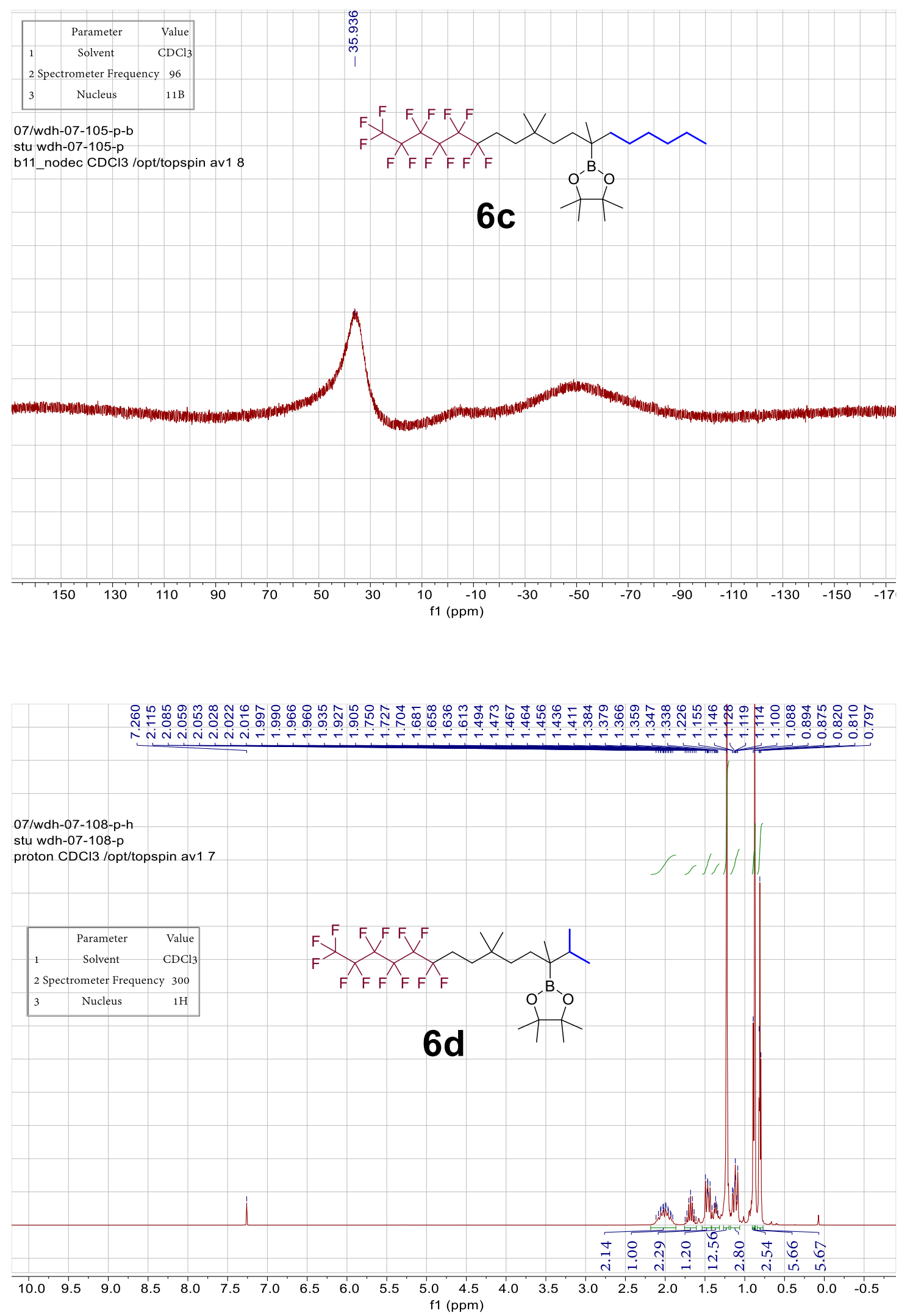


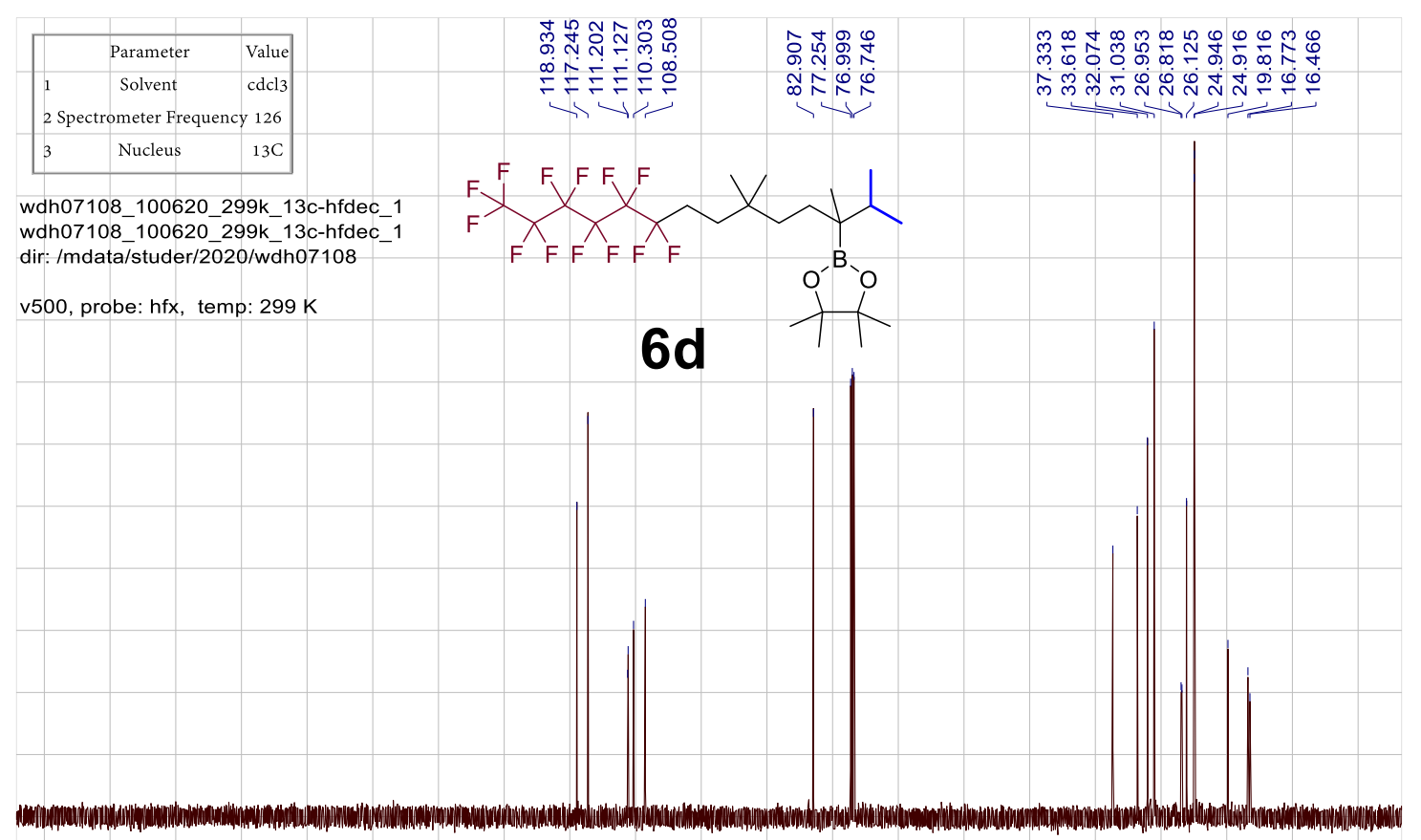

$\begin{array}{lllllllllllllllllllll}1 & 190 & 180 & 170 & 160 & 150 & 140 & 130 & 120 & 110 & \begin{array}{c}100 \\ \mathrm{f} 1(\mathrm{ppm})\end{array} & 90 & 70 & 60 & 50 & 40 & 30 & 20 & 10 & 0\end{array}$

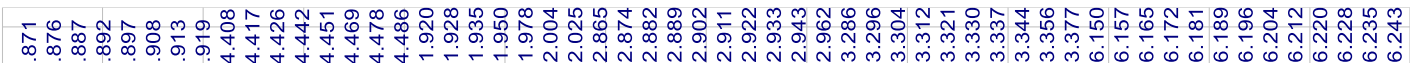

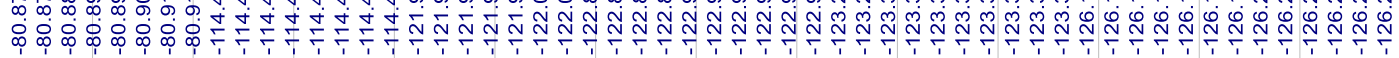

wdh07108 100620 299k 19f-bb-hdec

wdh07108_100620_299k_19f-bb-hdec_1

dir: /mdata/studer/2020/wdh07108

v500, probe: $\mathrm{hfx}$, temp: $299 \mathrm{~K}$

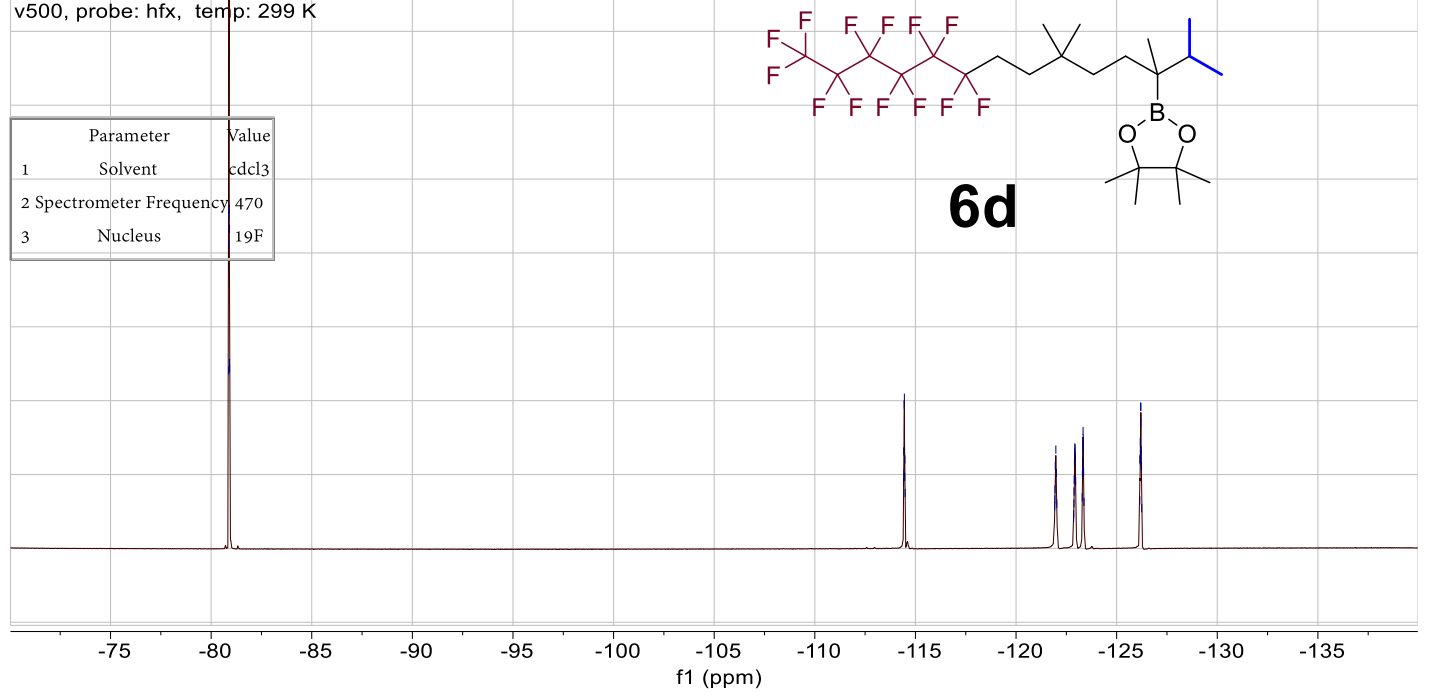



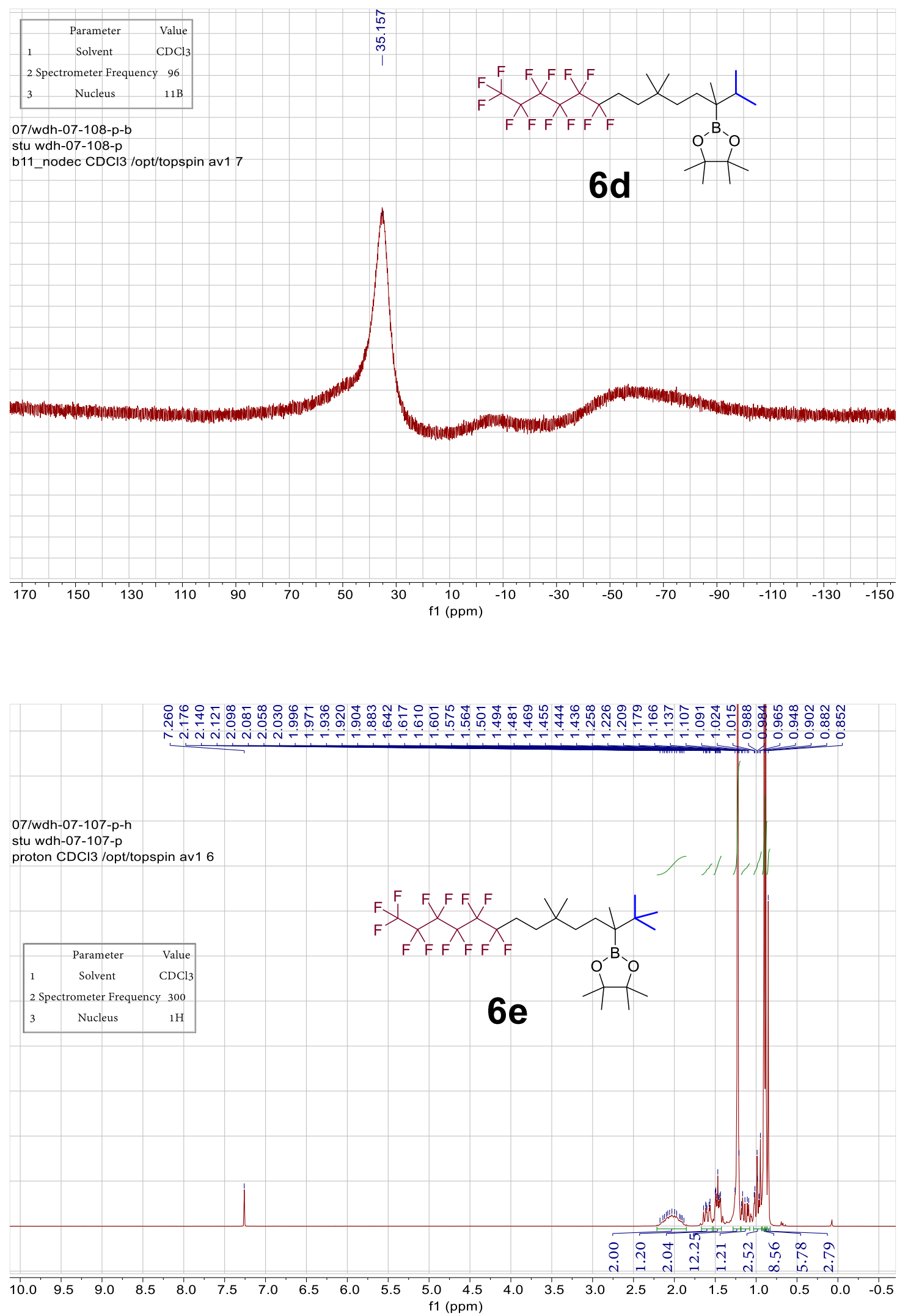

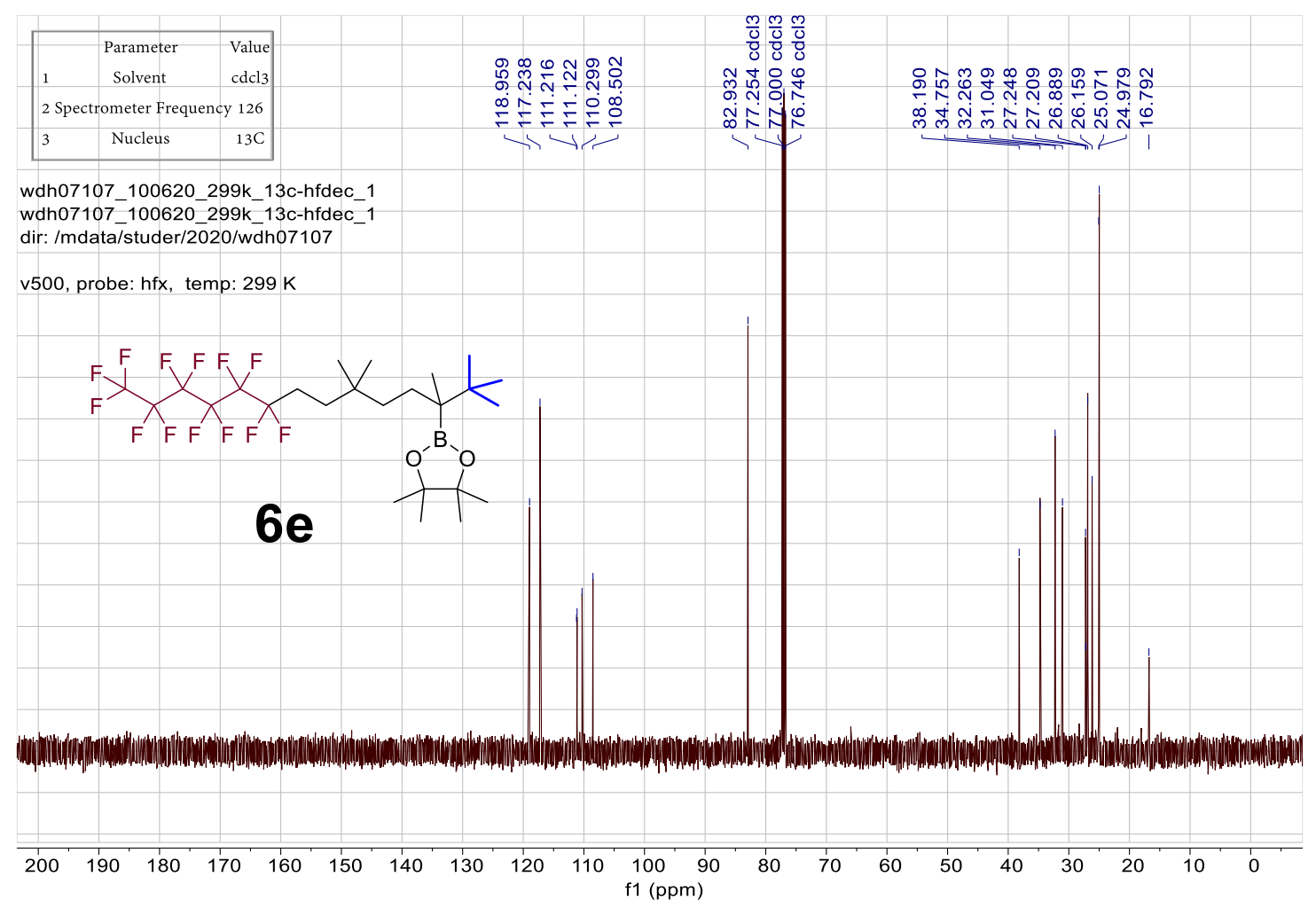

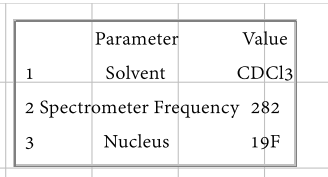

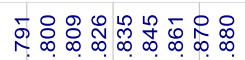

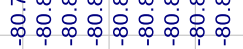

\section{7/wdh-07-107-p-f}

stu wdh-07-107-p

f19cpd CDCl3 /opt/topspin av1 6

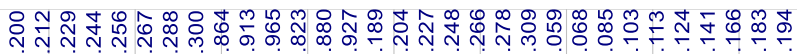

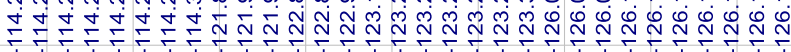



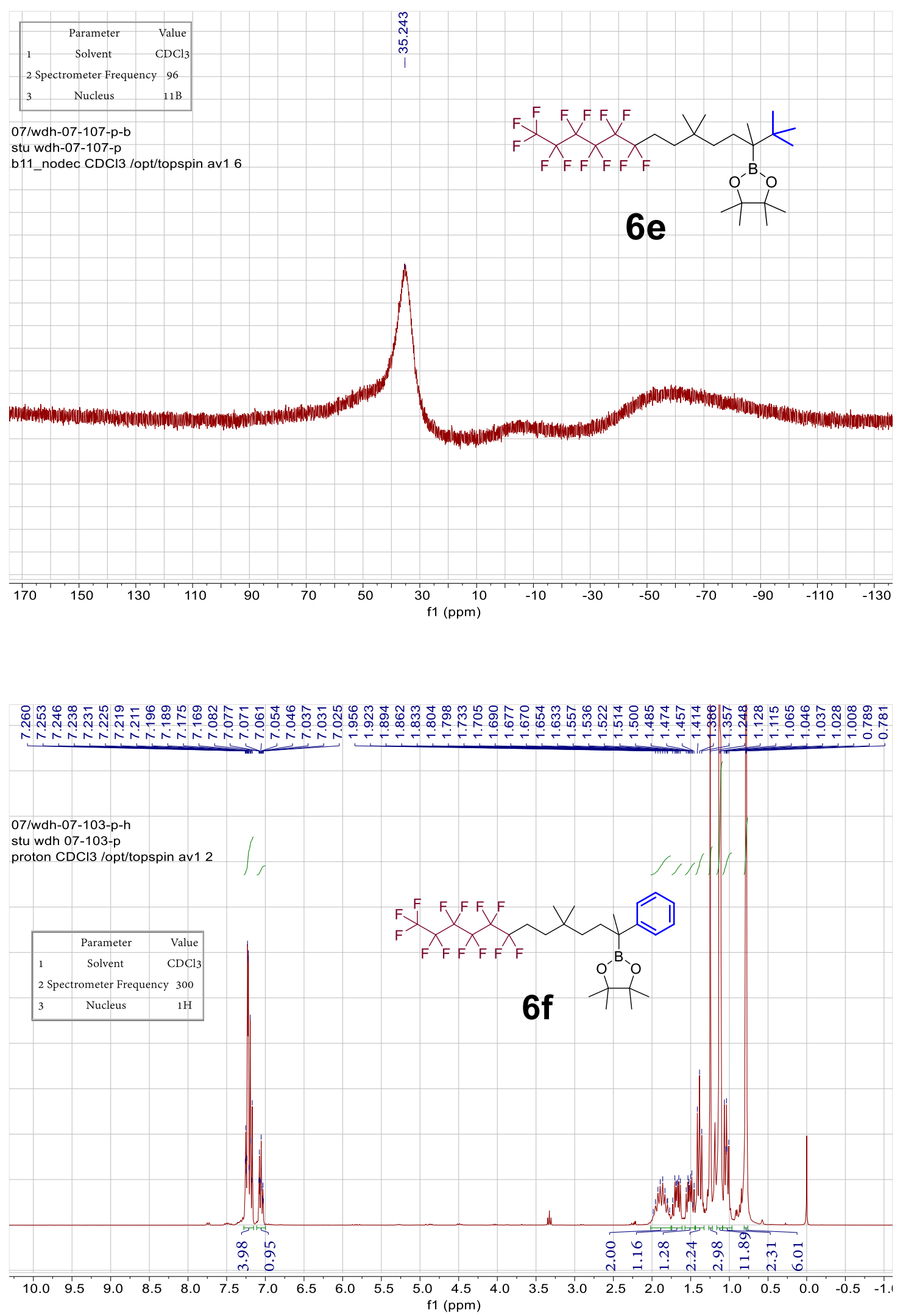

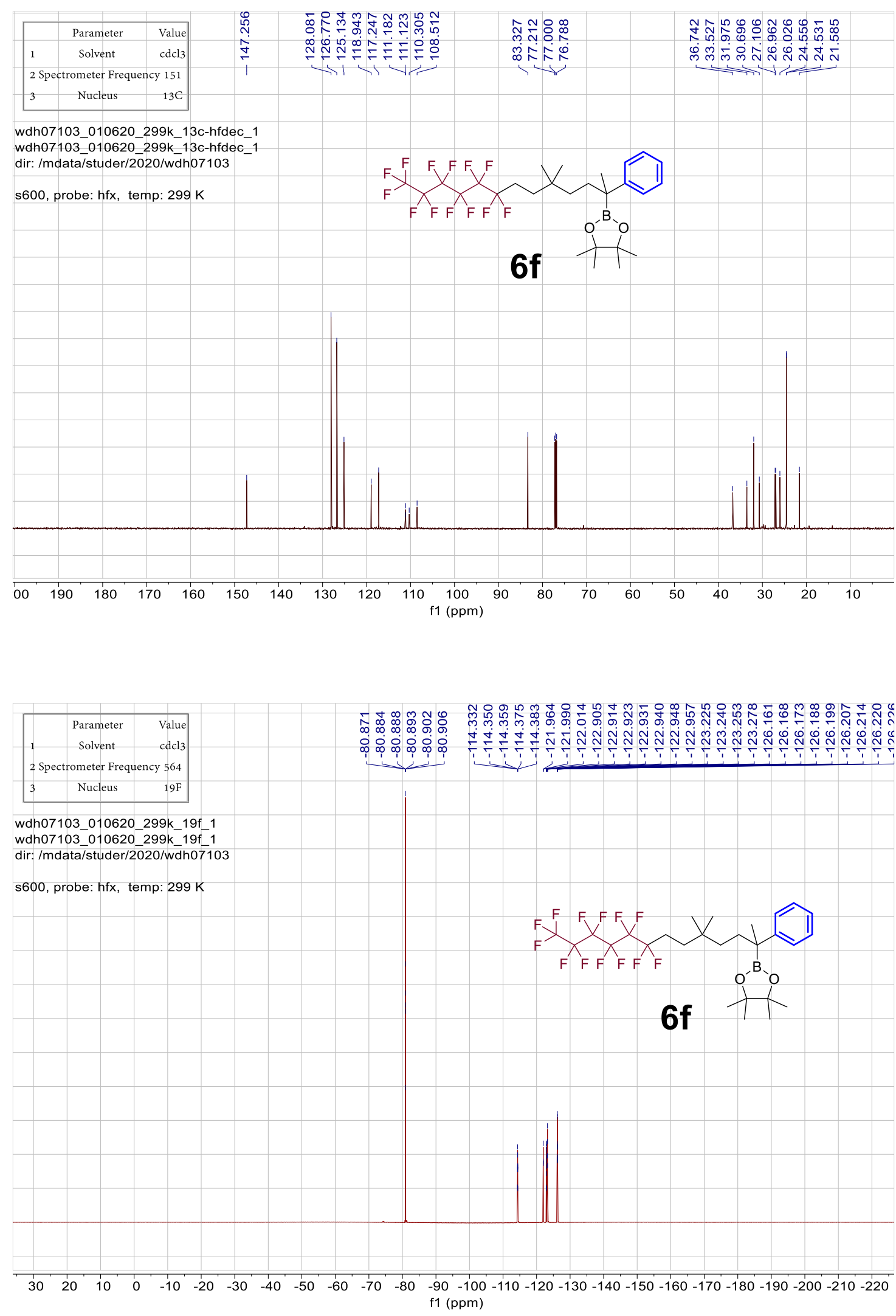

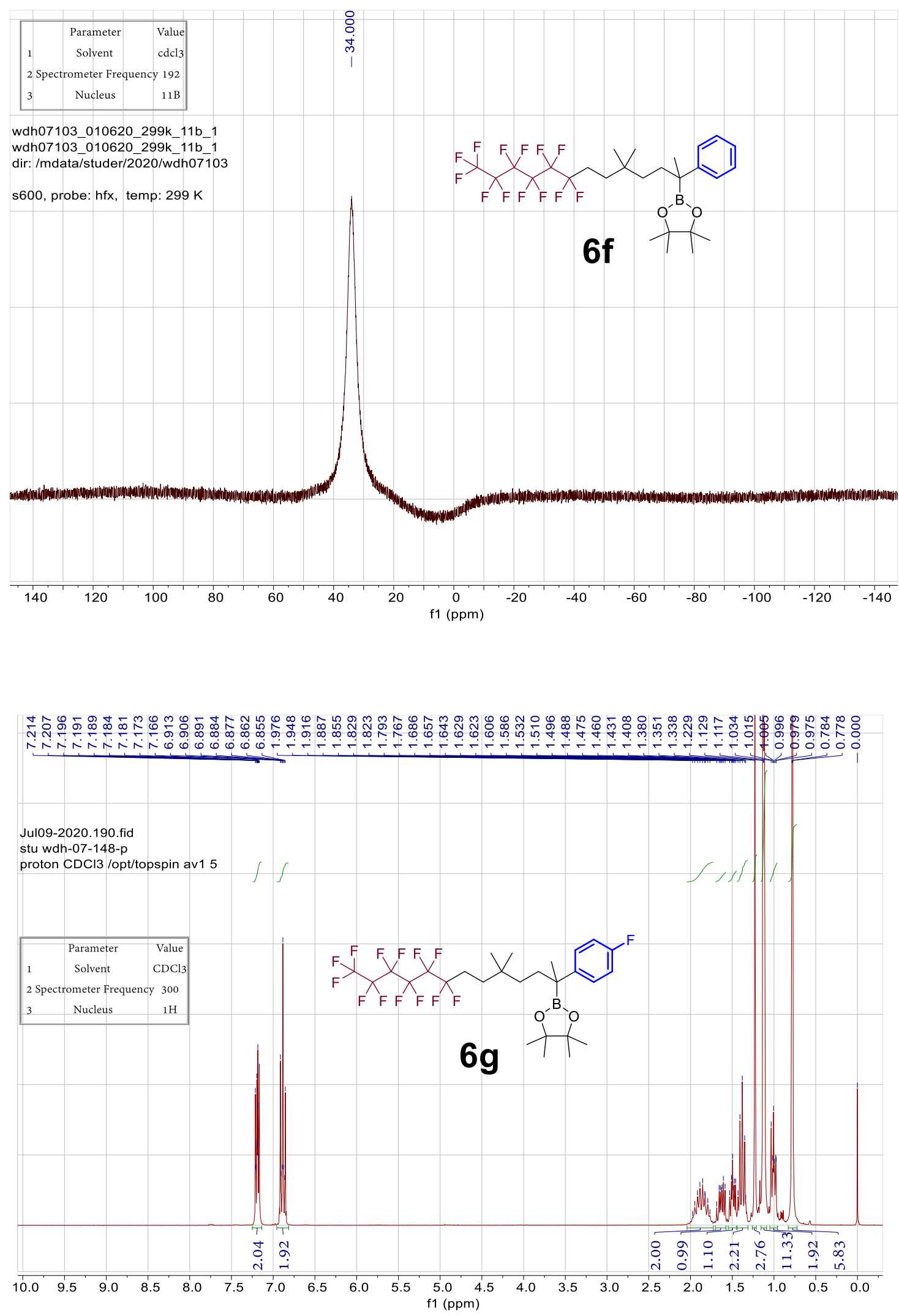


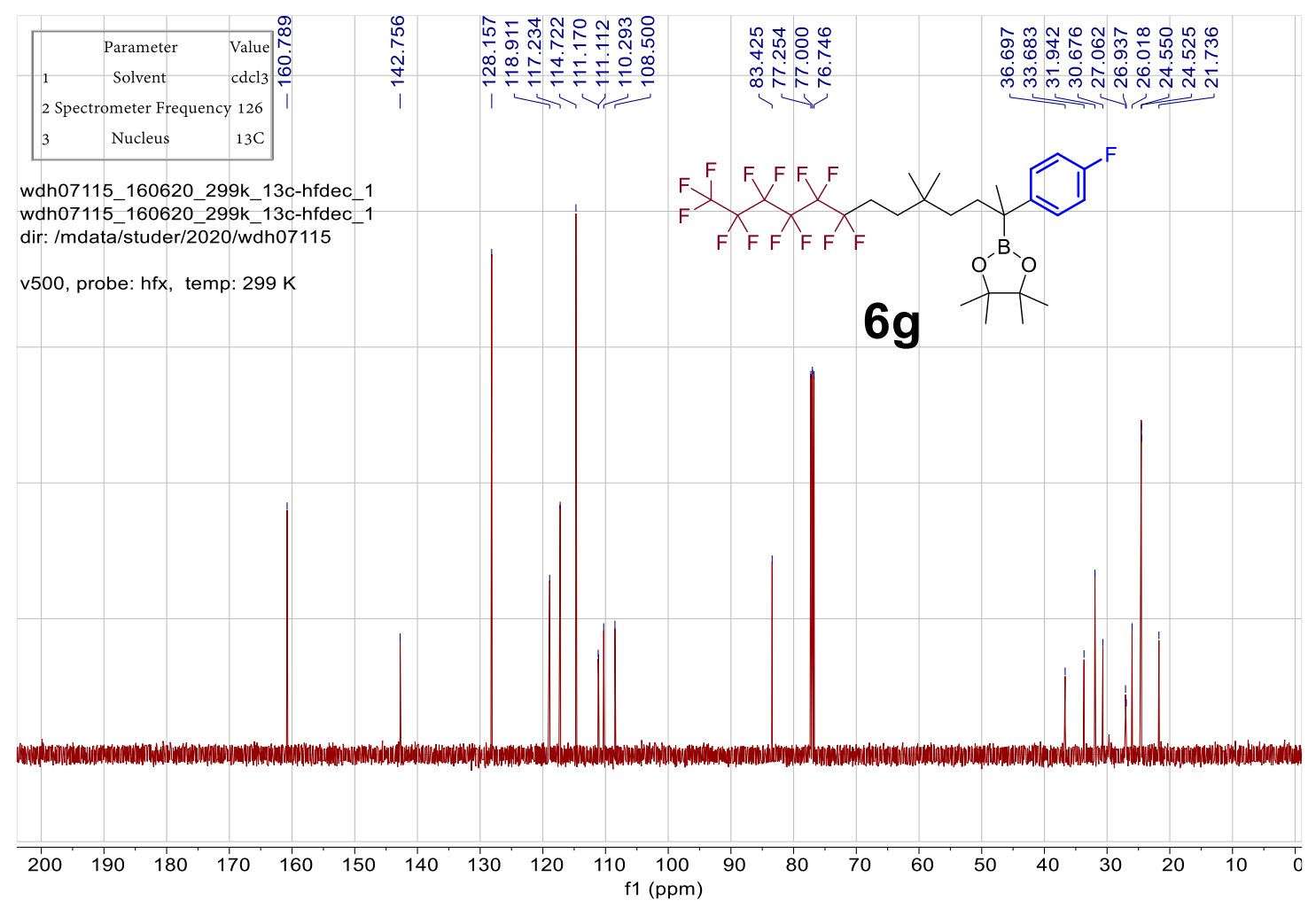

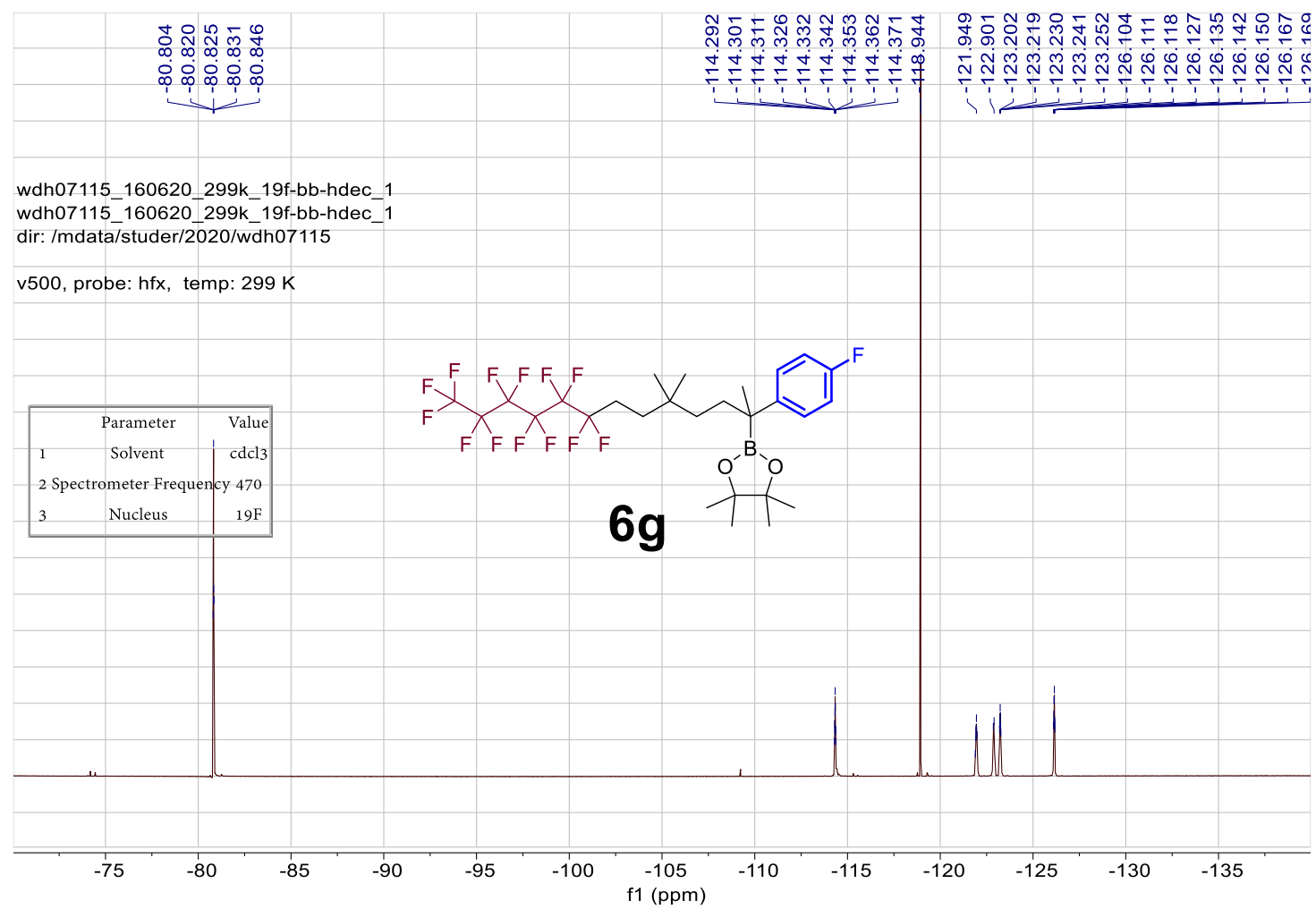



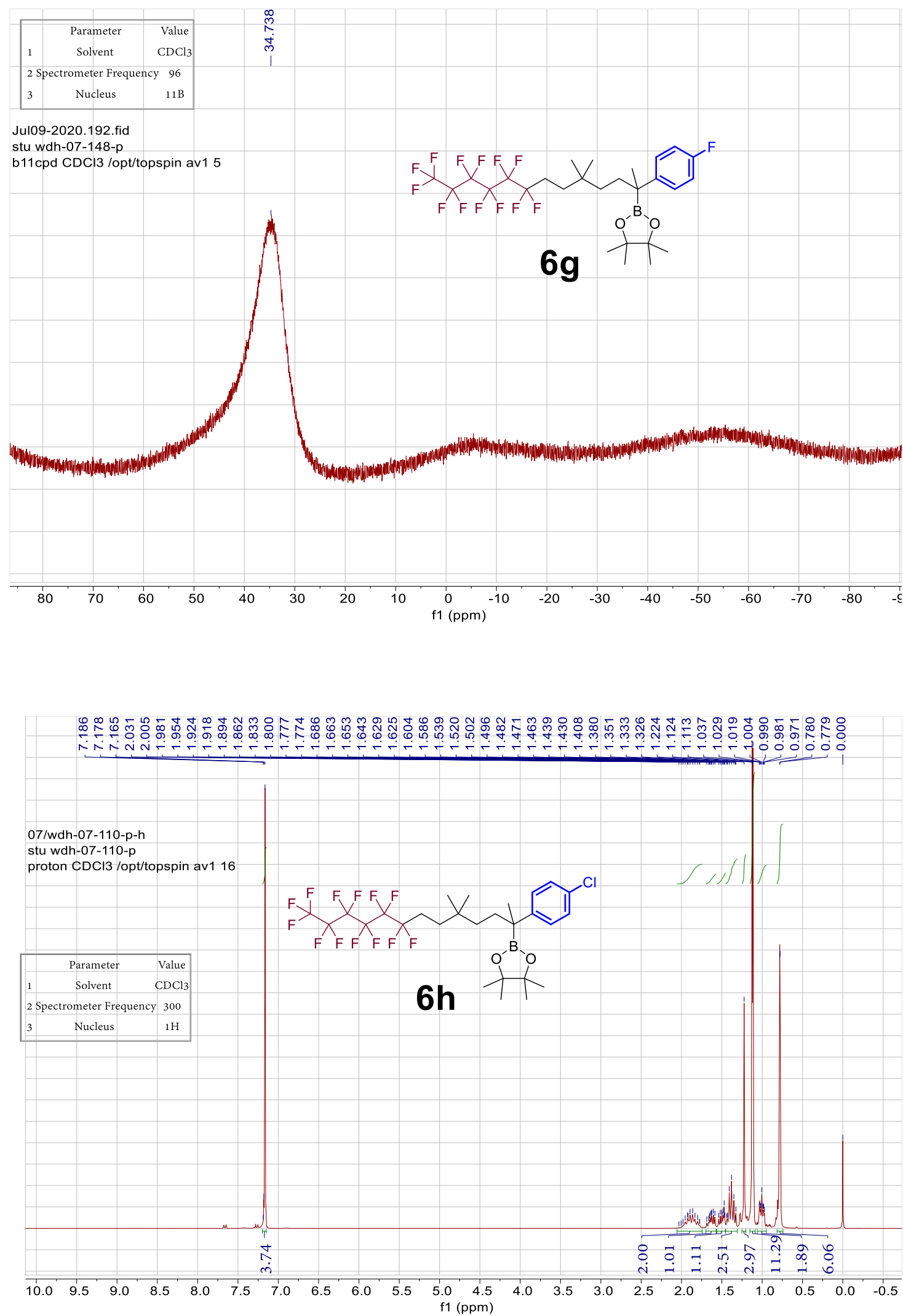

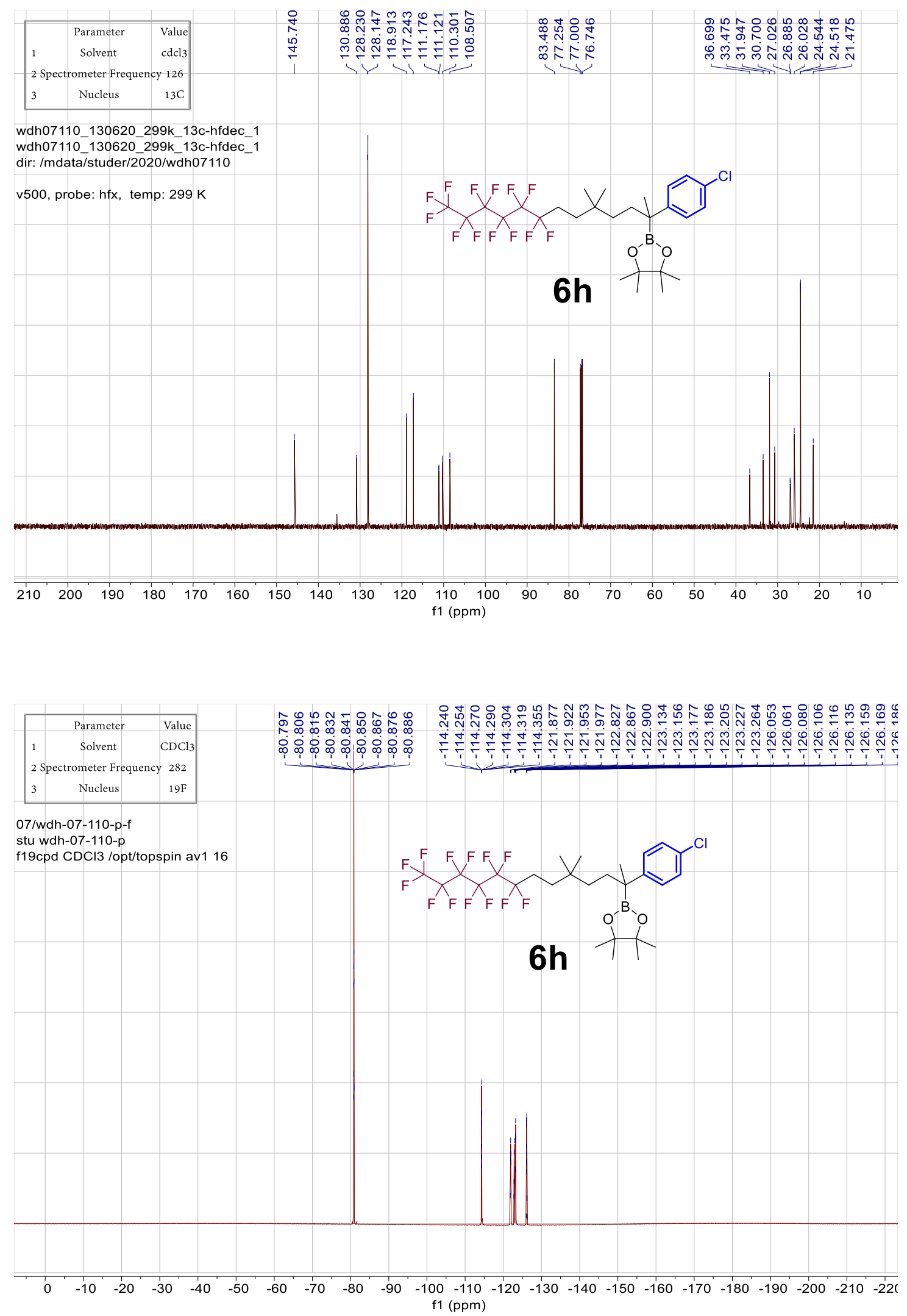

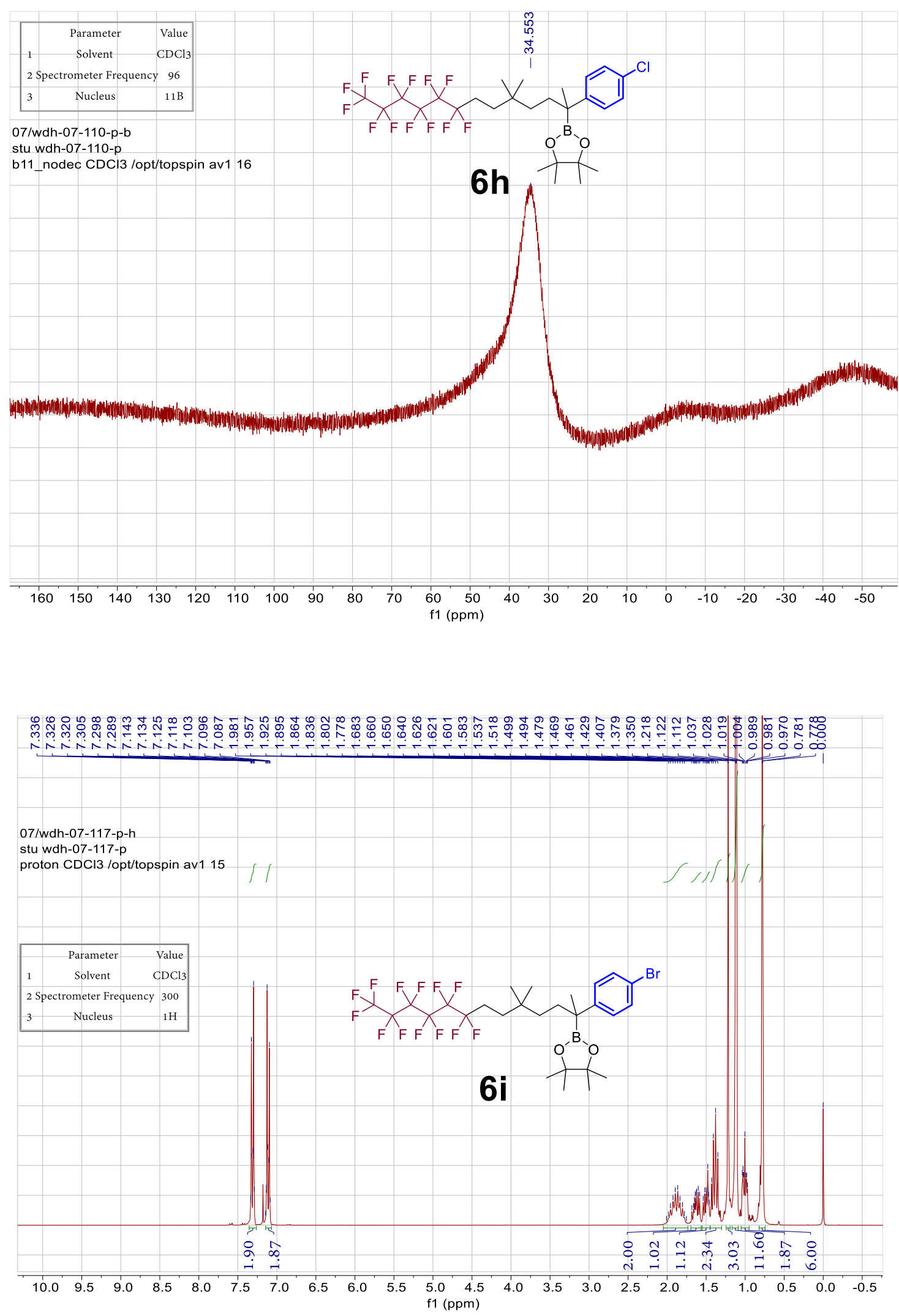


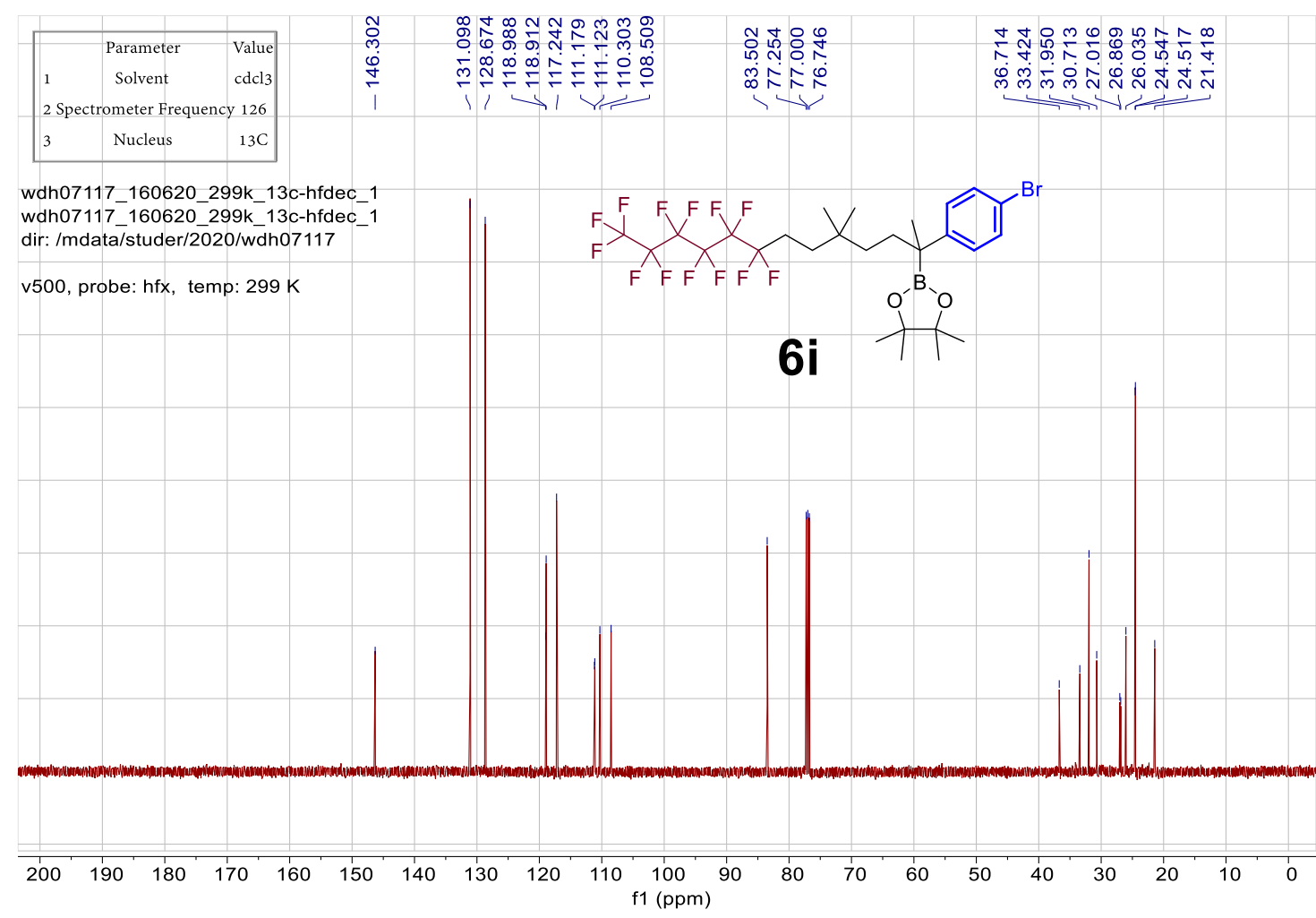

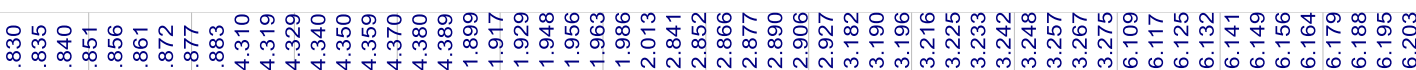

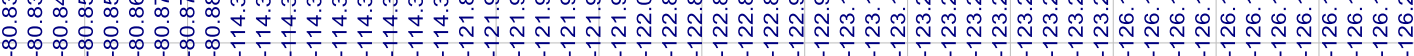

wdh07117_160620_299k_19f-bb-hdec

wdh07117 160620_299k_19f-bb-hdec 1

dir: /mdata/studer/2020/wdh07117

v500, probe: hfx, temp: $299 \mathrm{~K}$

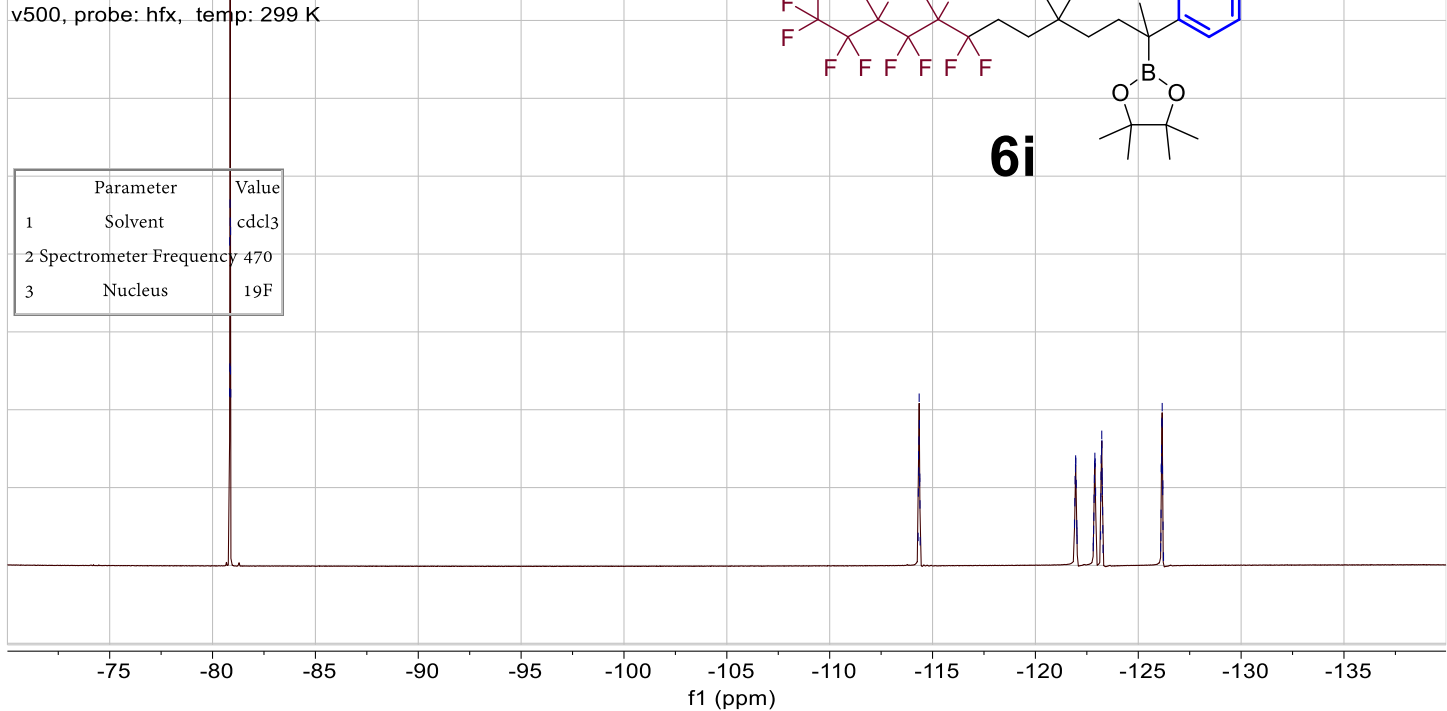



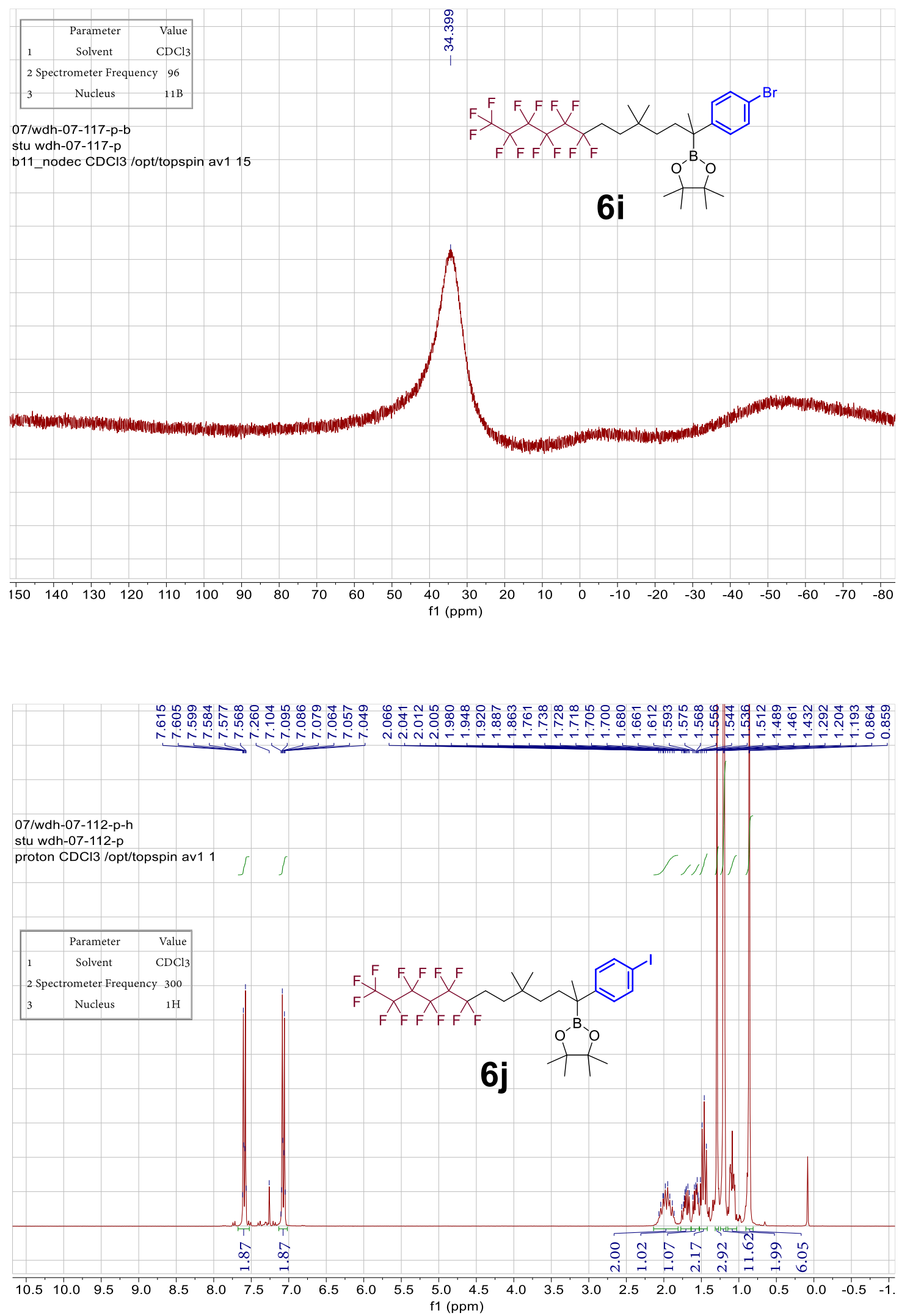

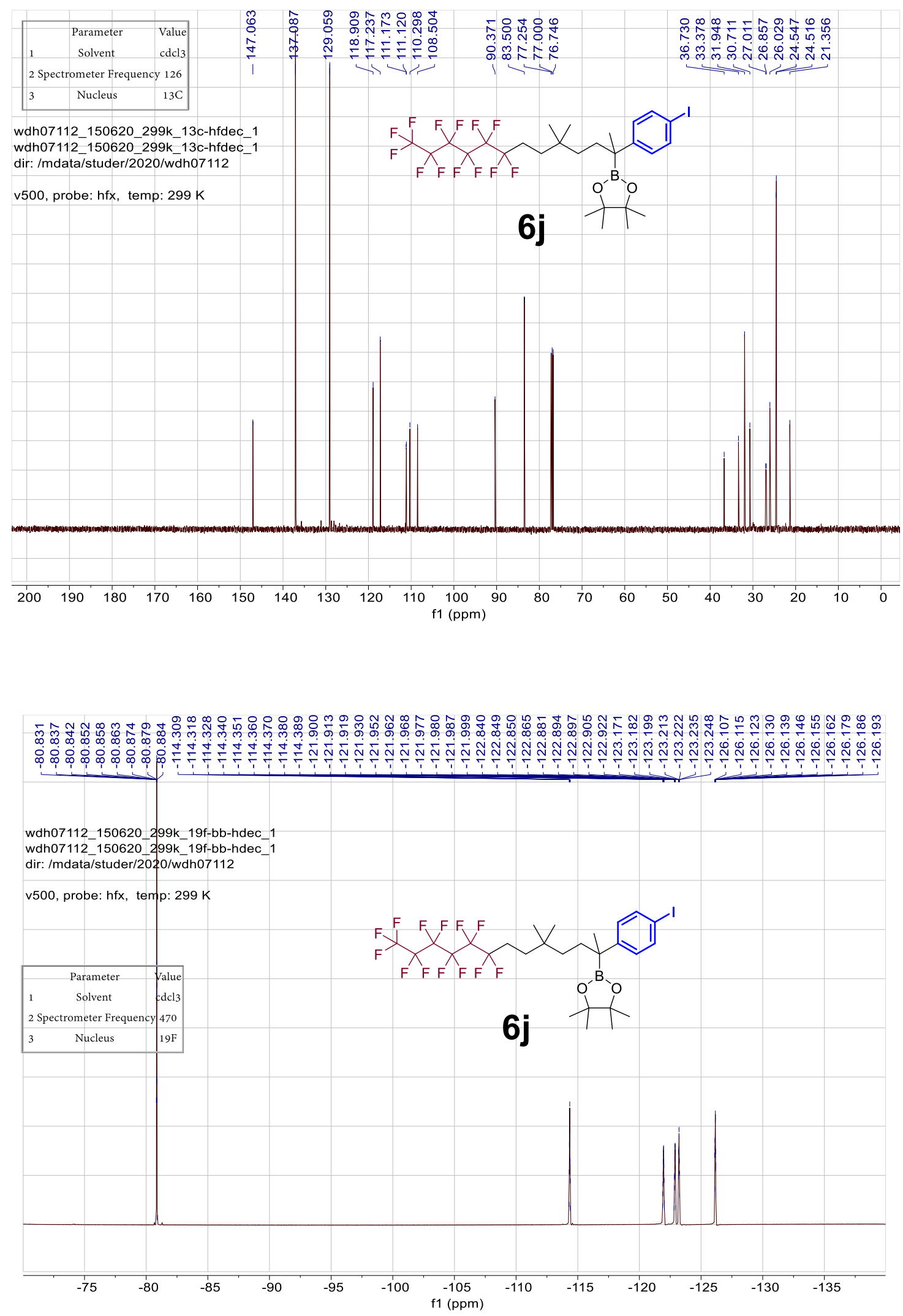

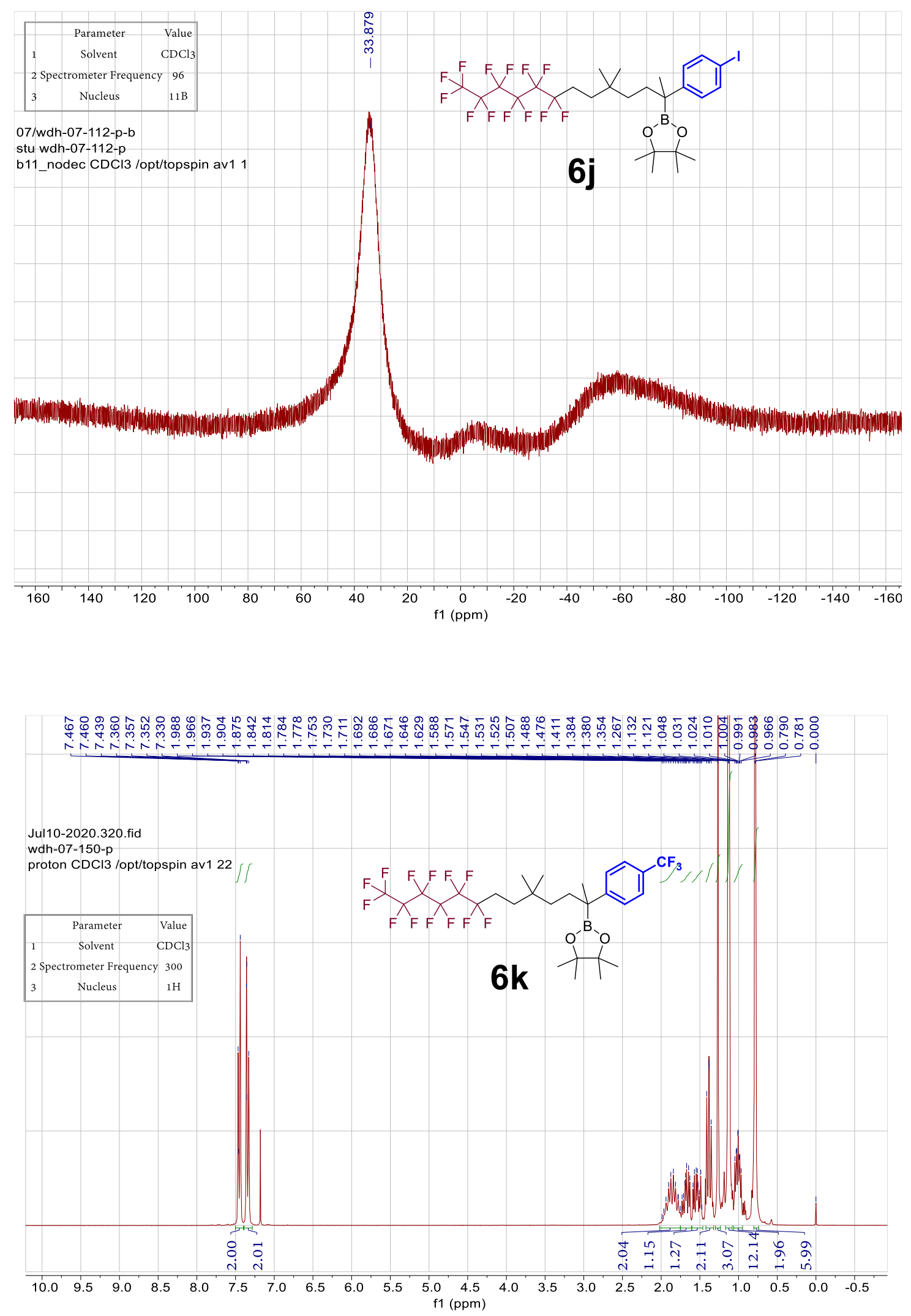


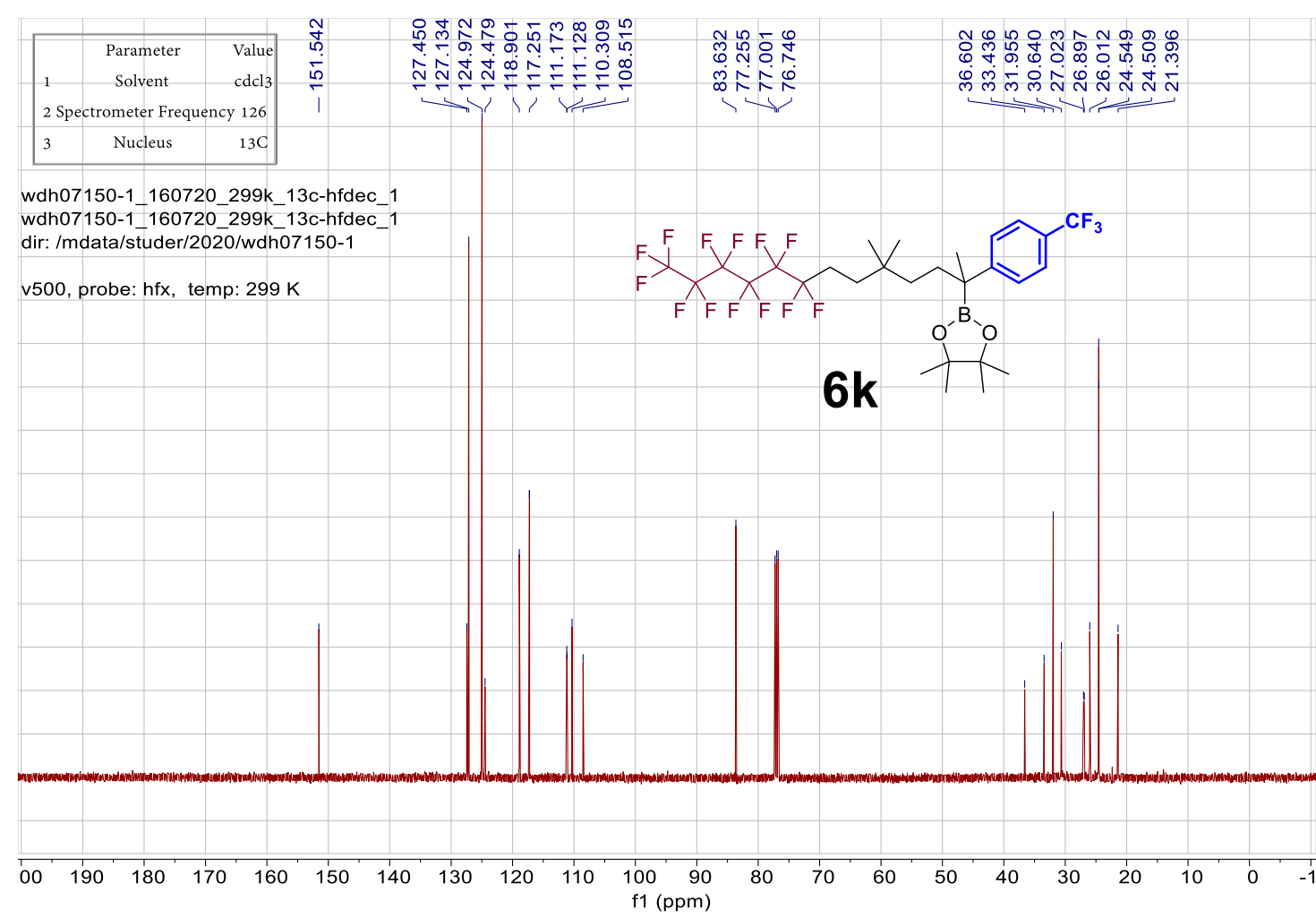

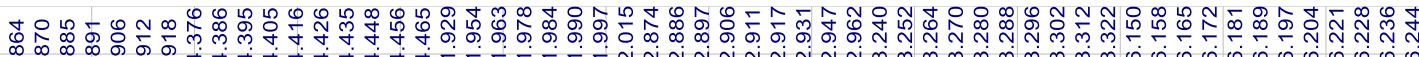

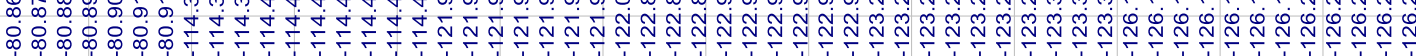

wdh07150-1_160720_299k_19f-bb-hdec_1

wdh07150-1_160720_299k_19f-bb-hdec_1

dir: /mdata/studer/2020/wdh07150-1

v500, probe: hfx, temp: $299 \mathrm{~K}$
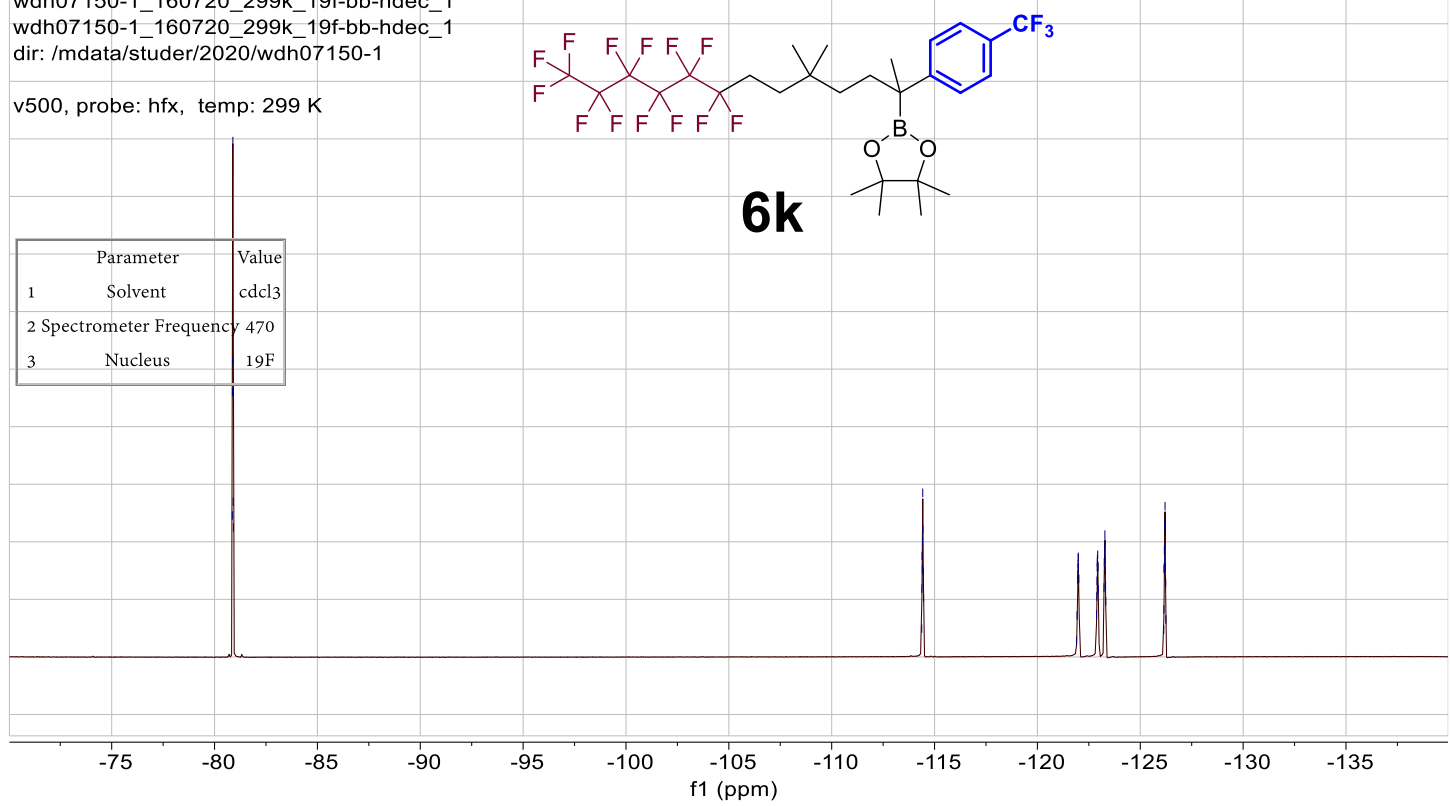

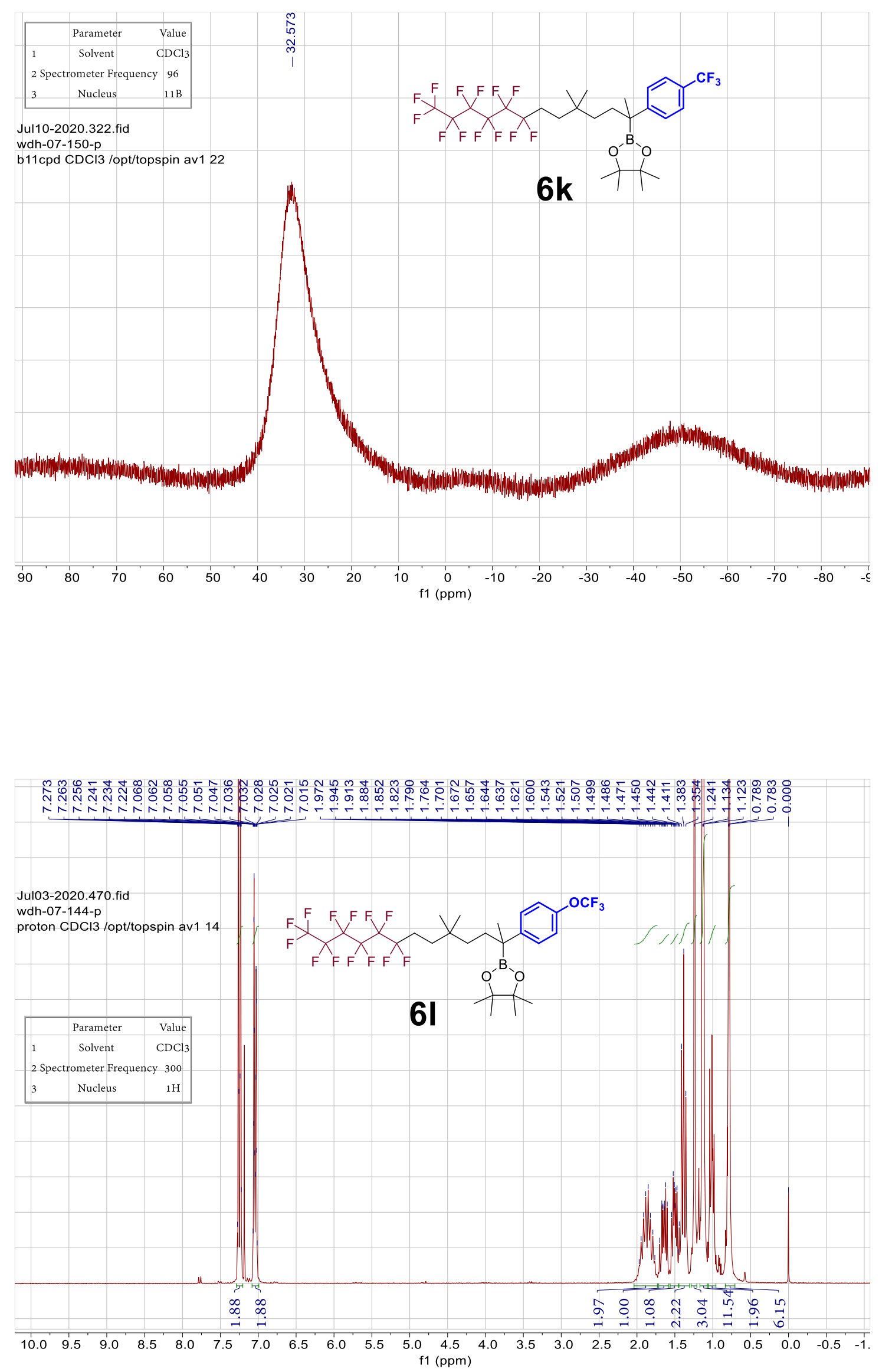


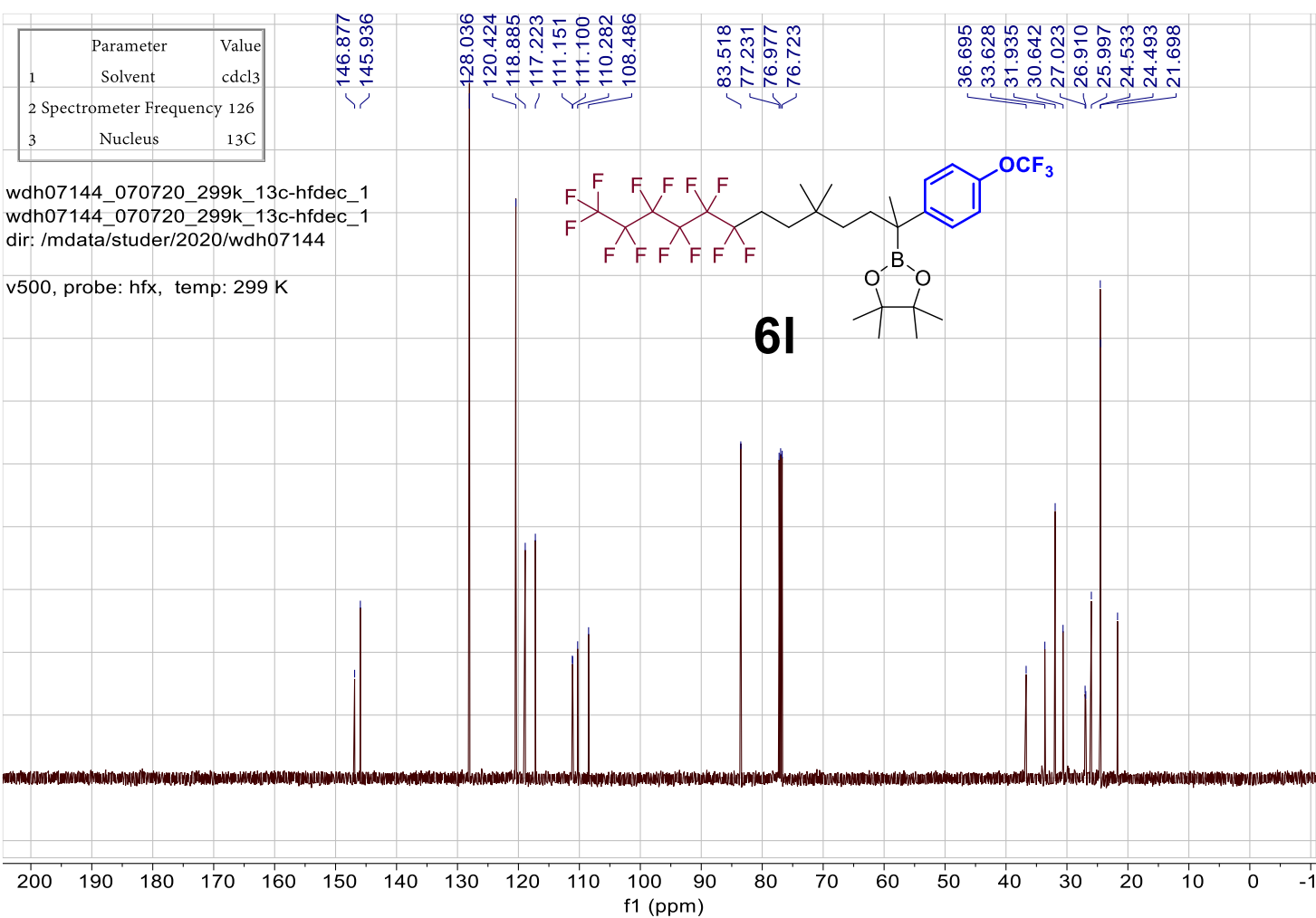

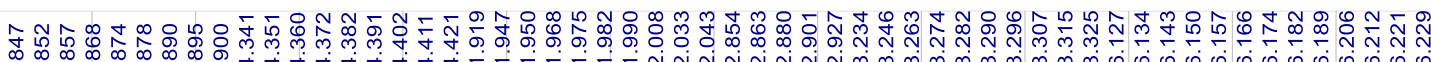

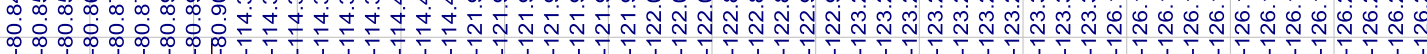

wdh07144_070720_299k_19f-bb-hdec_1

wdh07144_070720_299k_19f-bb-hdec_

dir:/mdata/studer/2020/wdh07144

v500, probe: hfx, temp: $299 \mathrm{~K}$

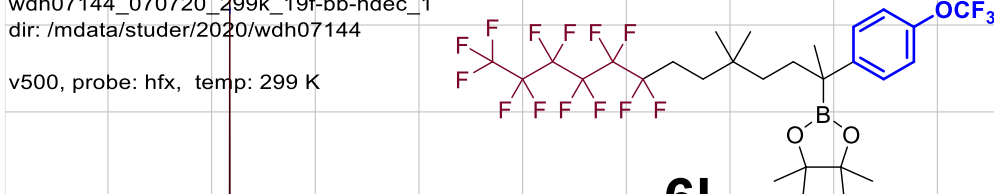

6I

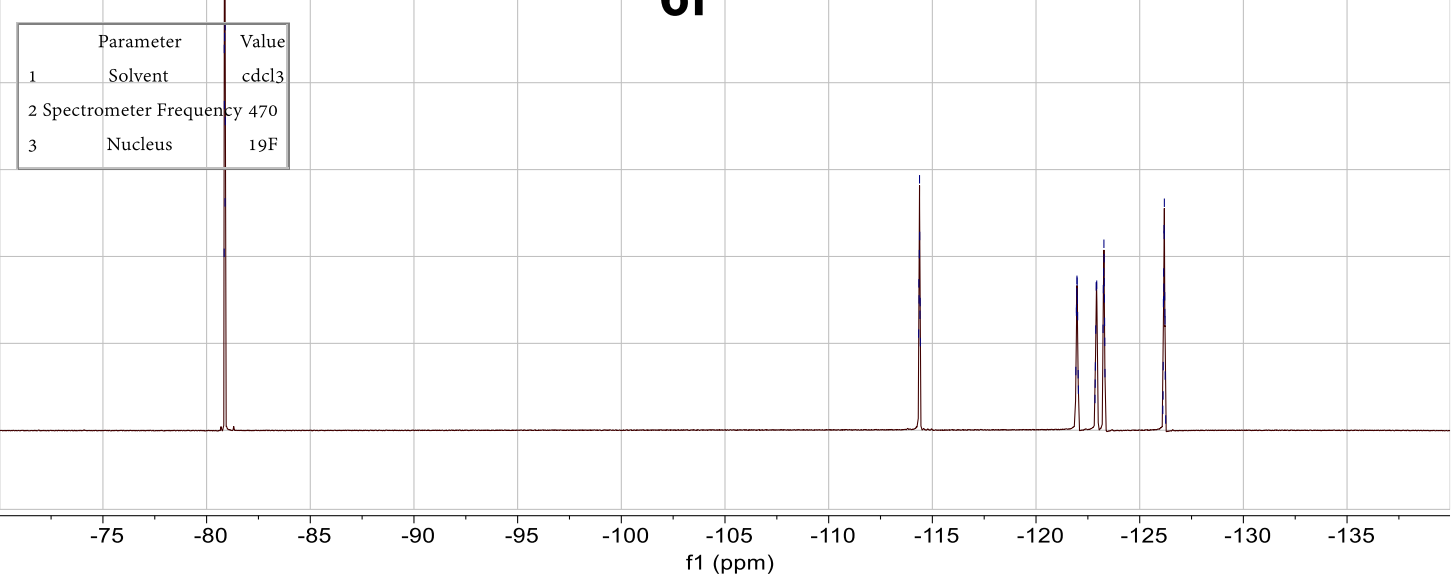



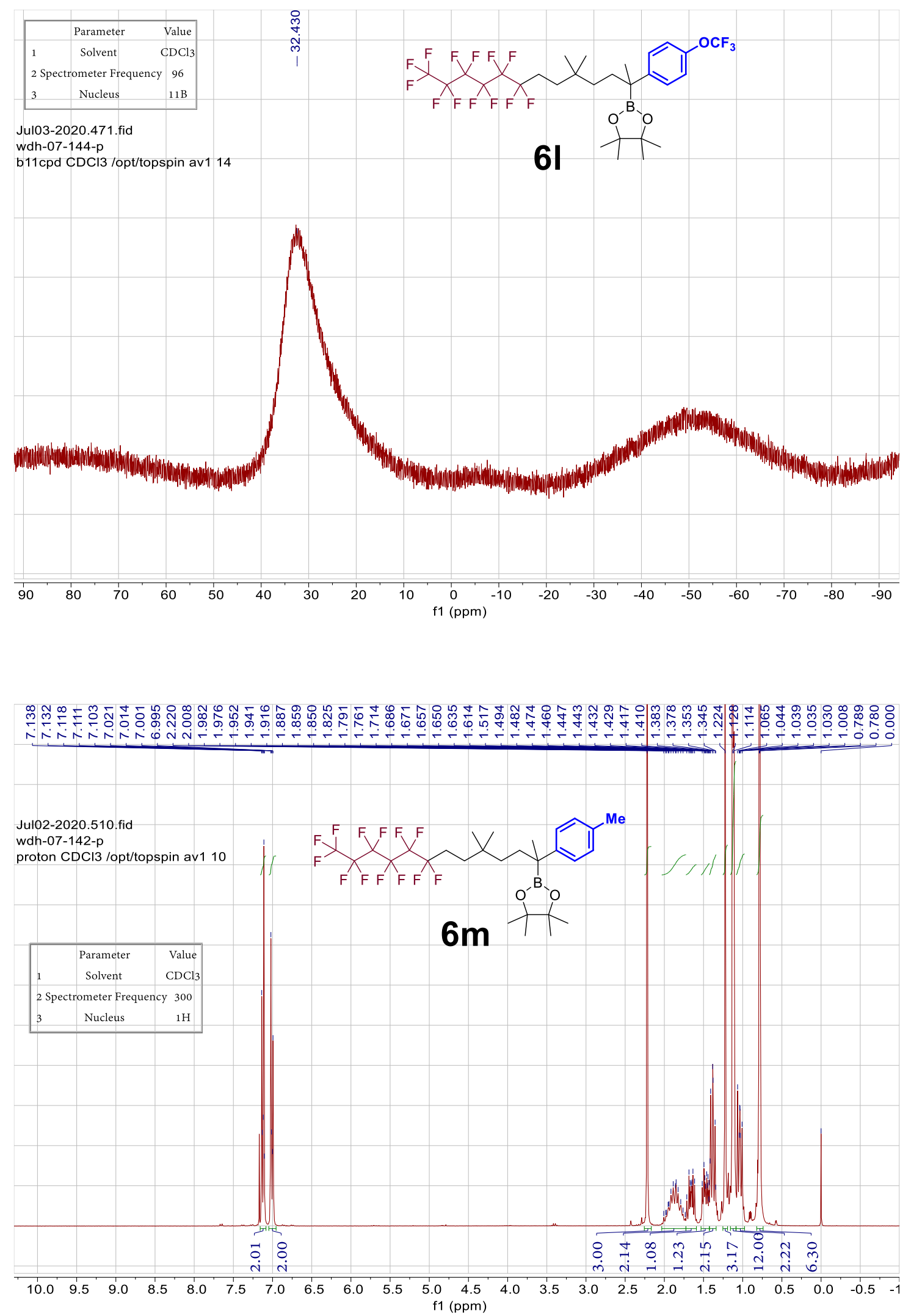


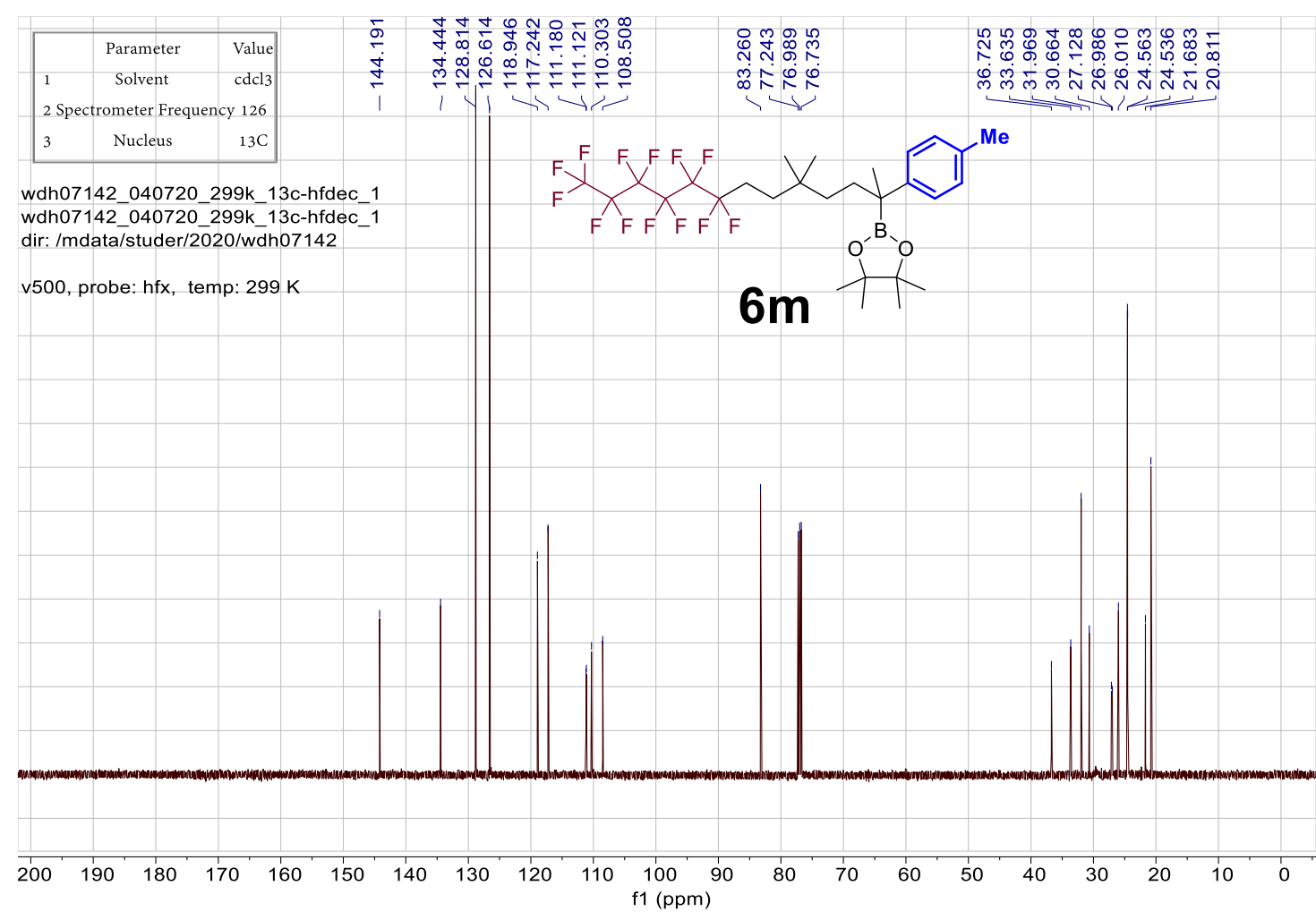

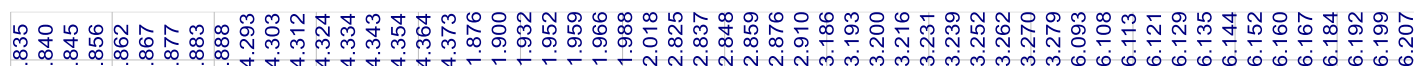

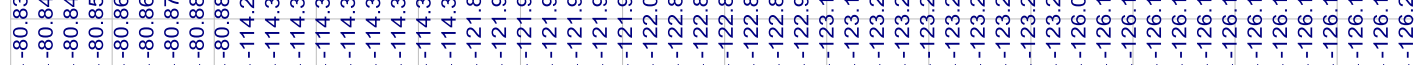

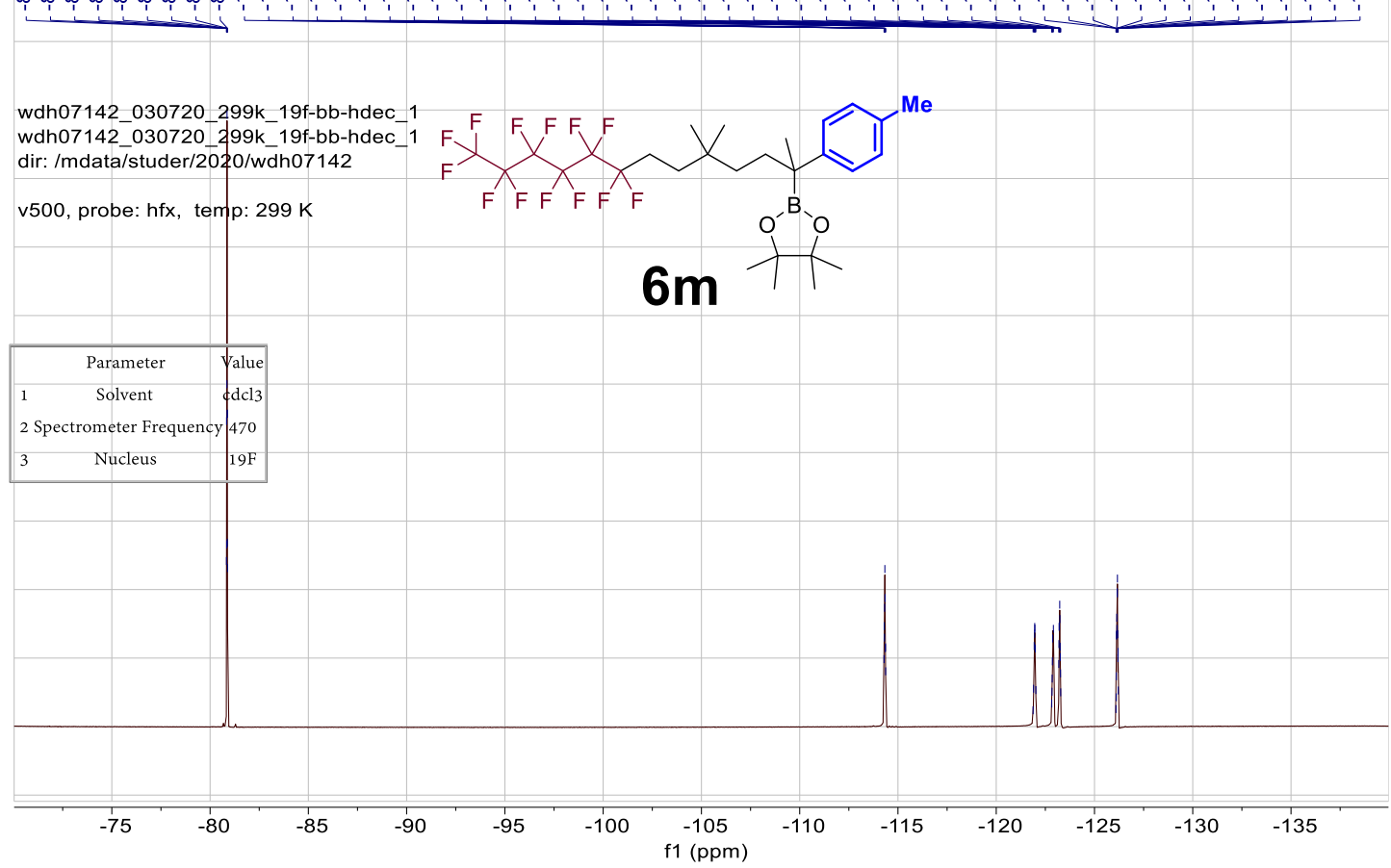



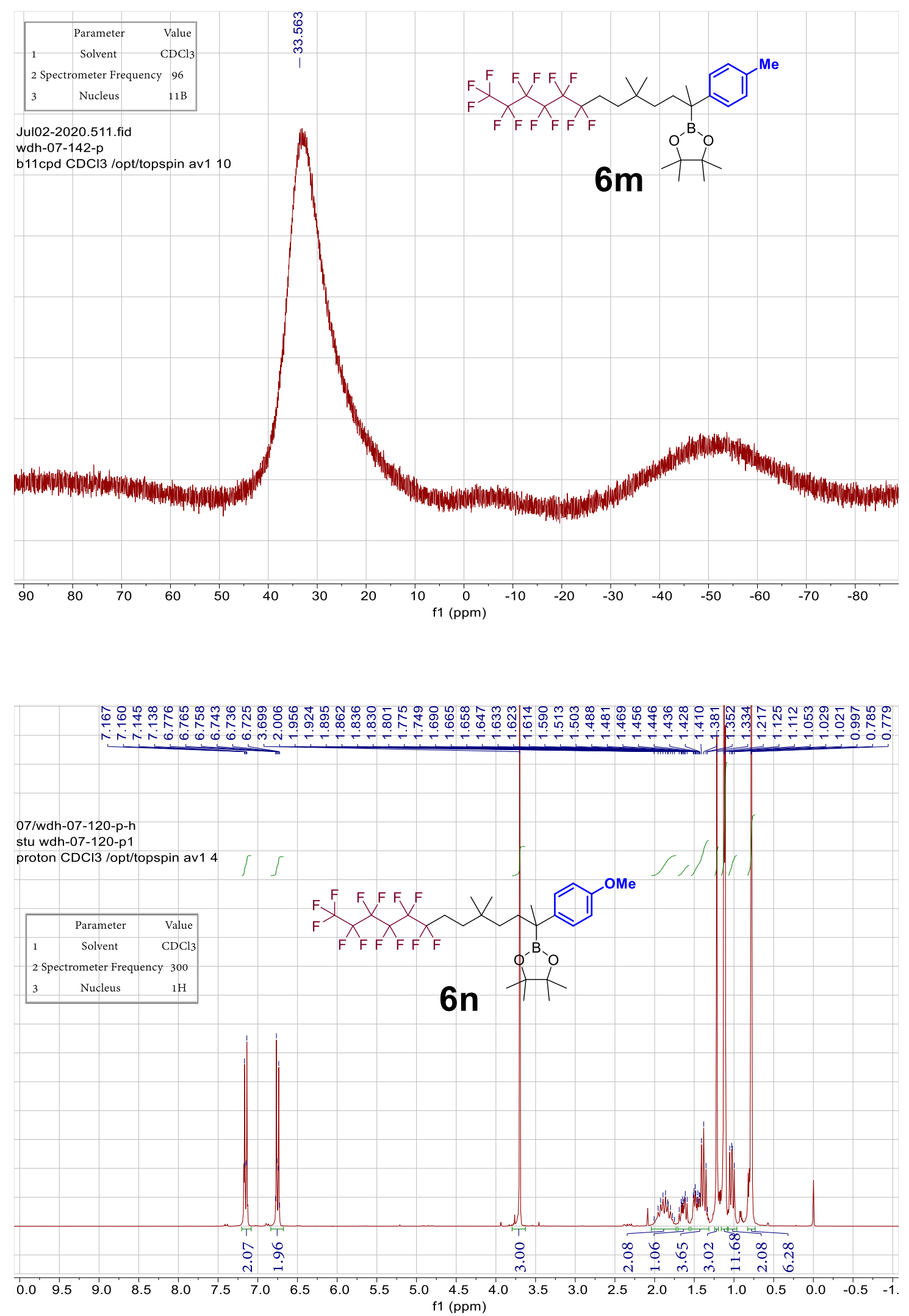


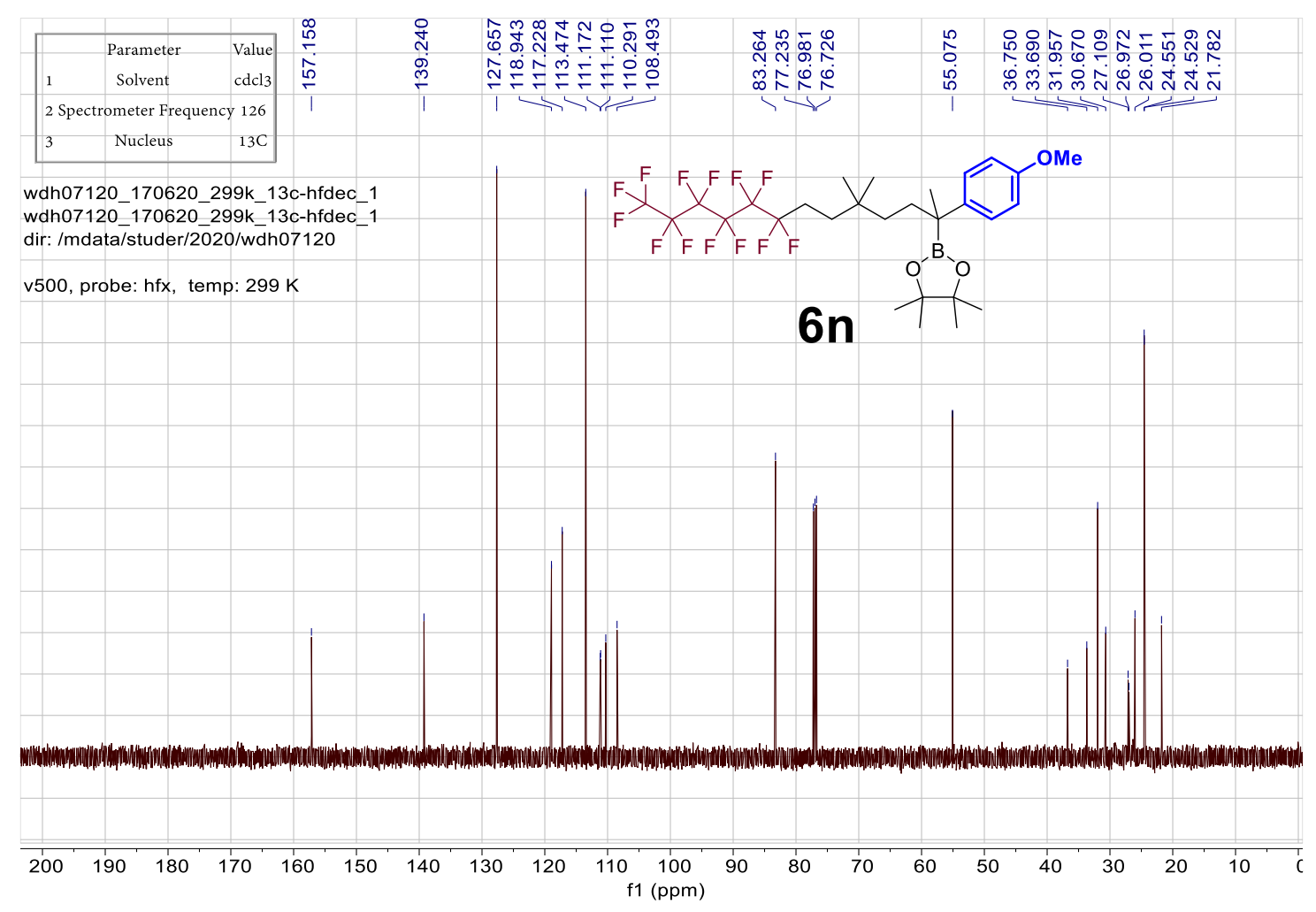

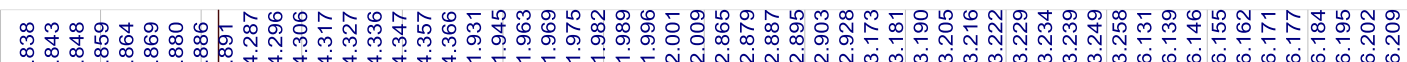

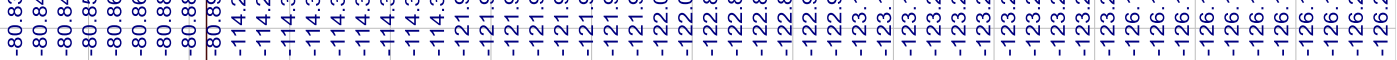

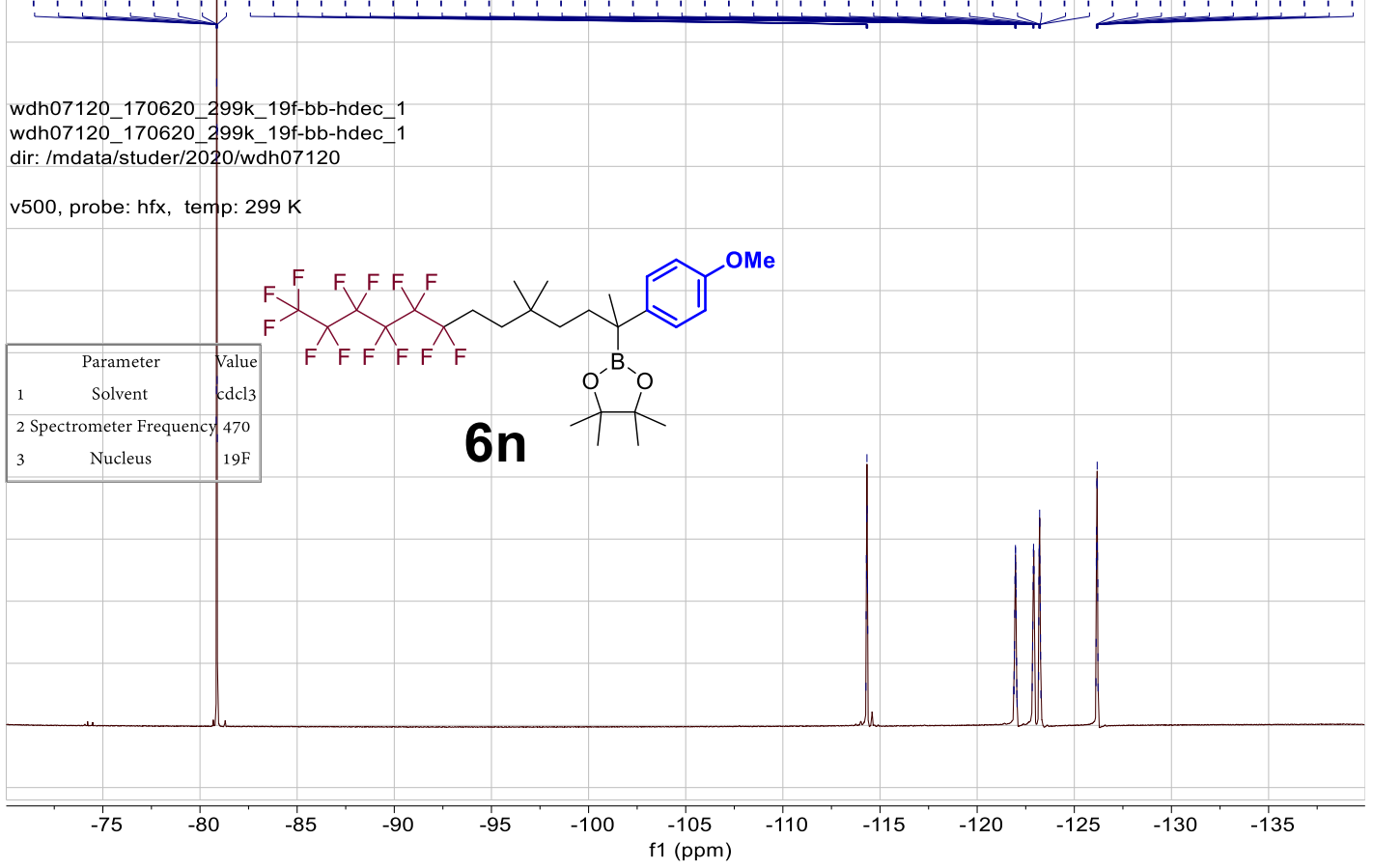



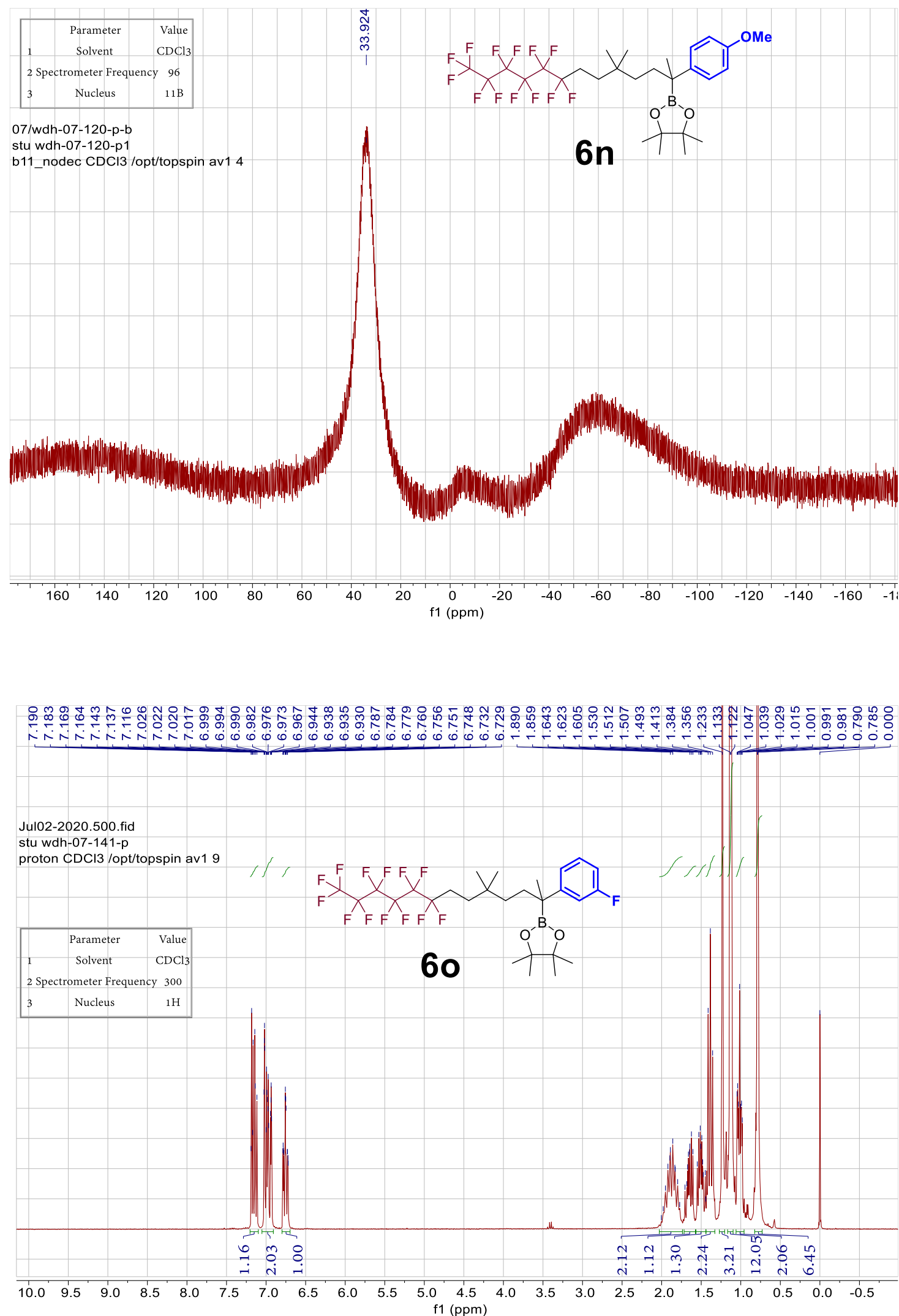


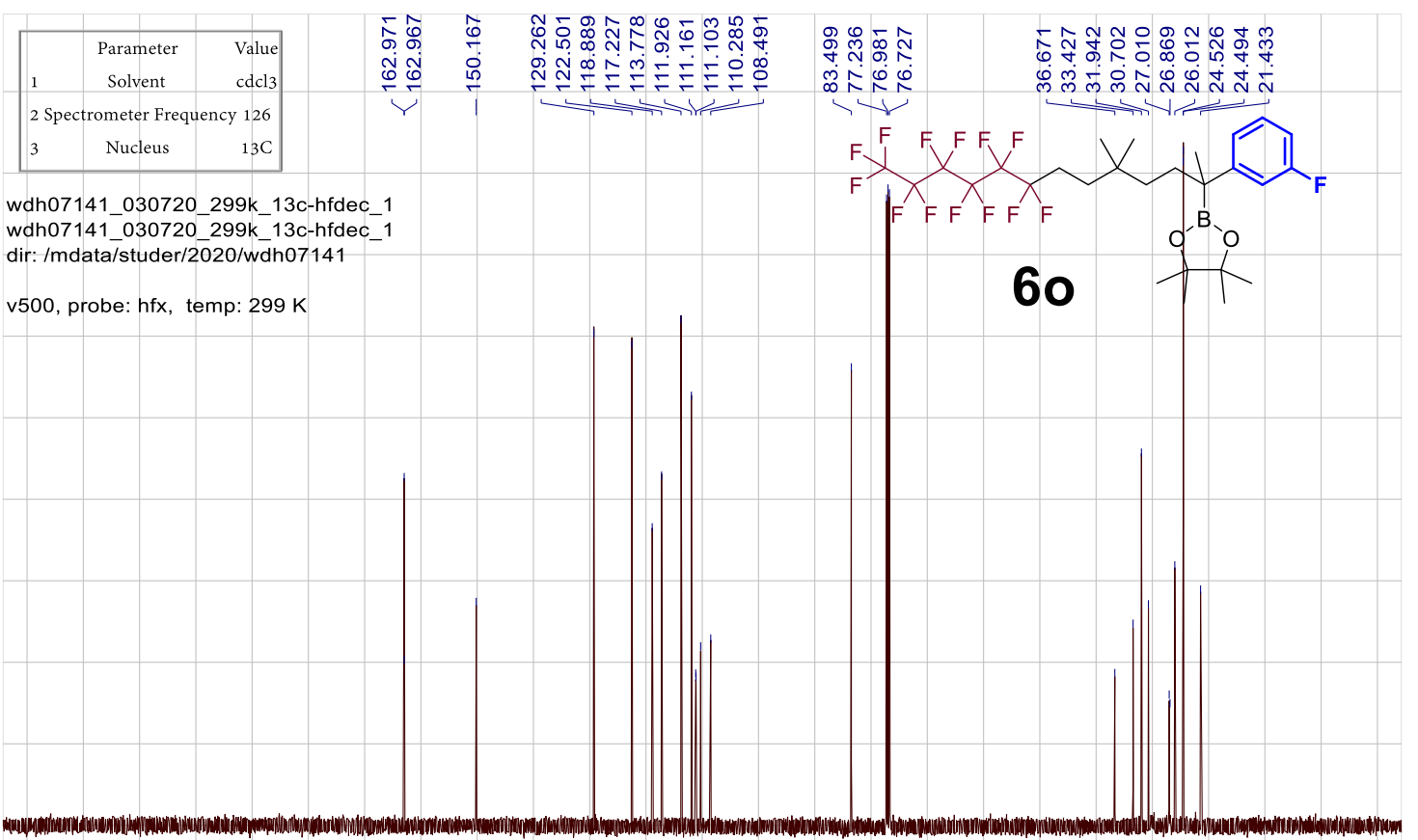

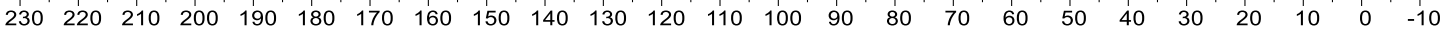
$\mathrm{f} 1(\mathrm{ppm})$

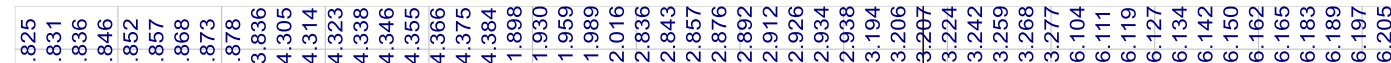

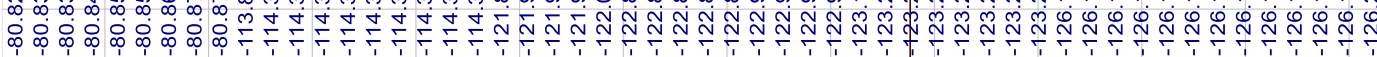

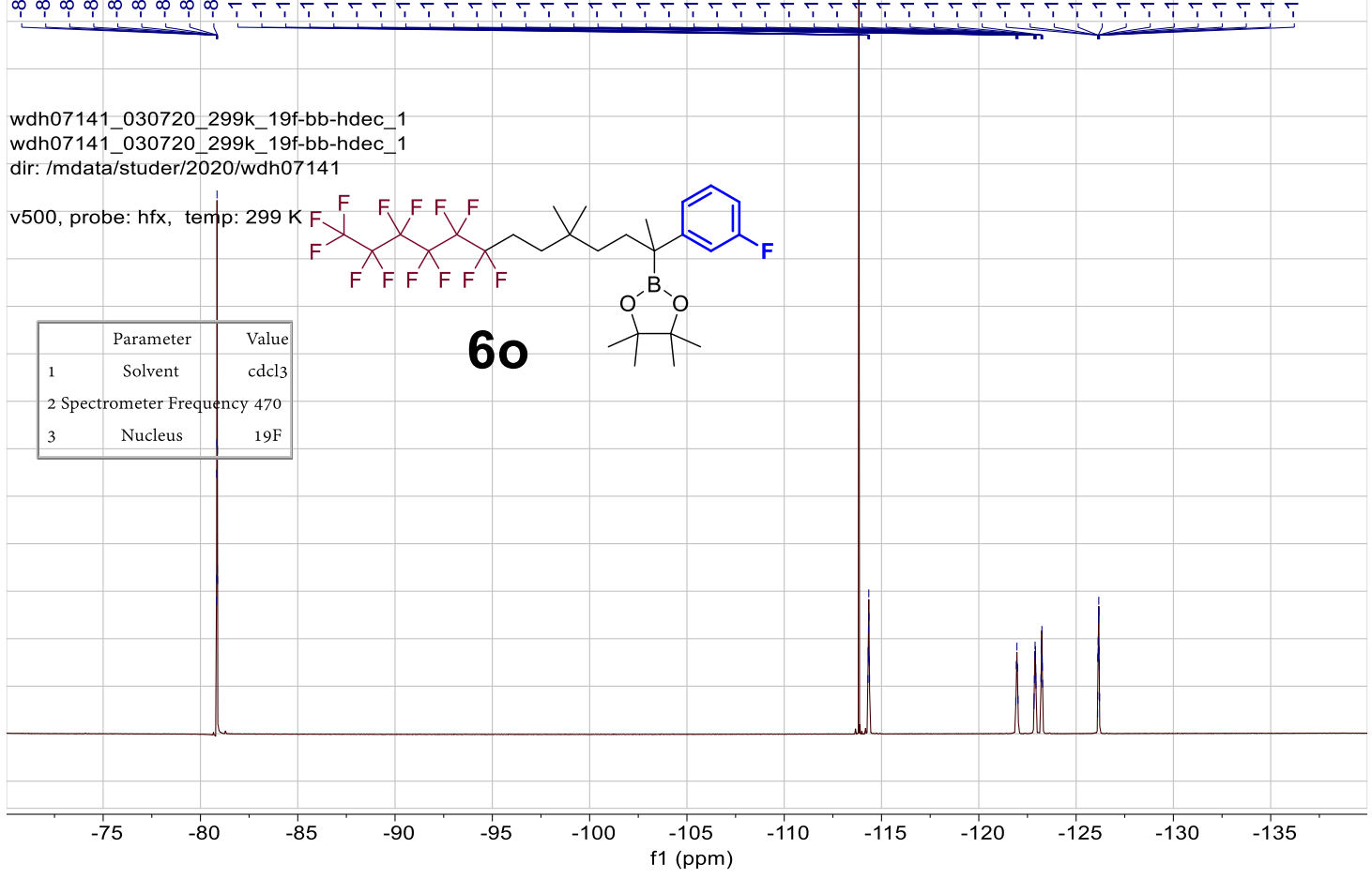



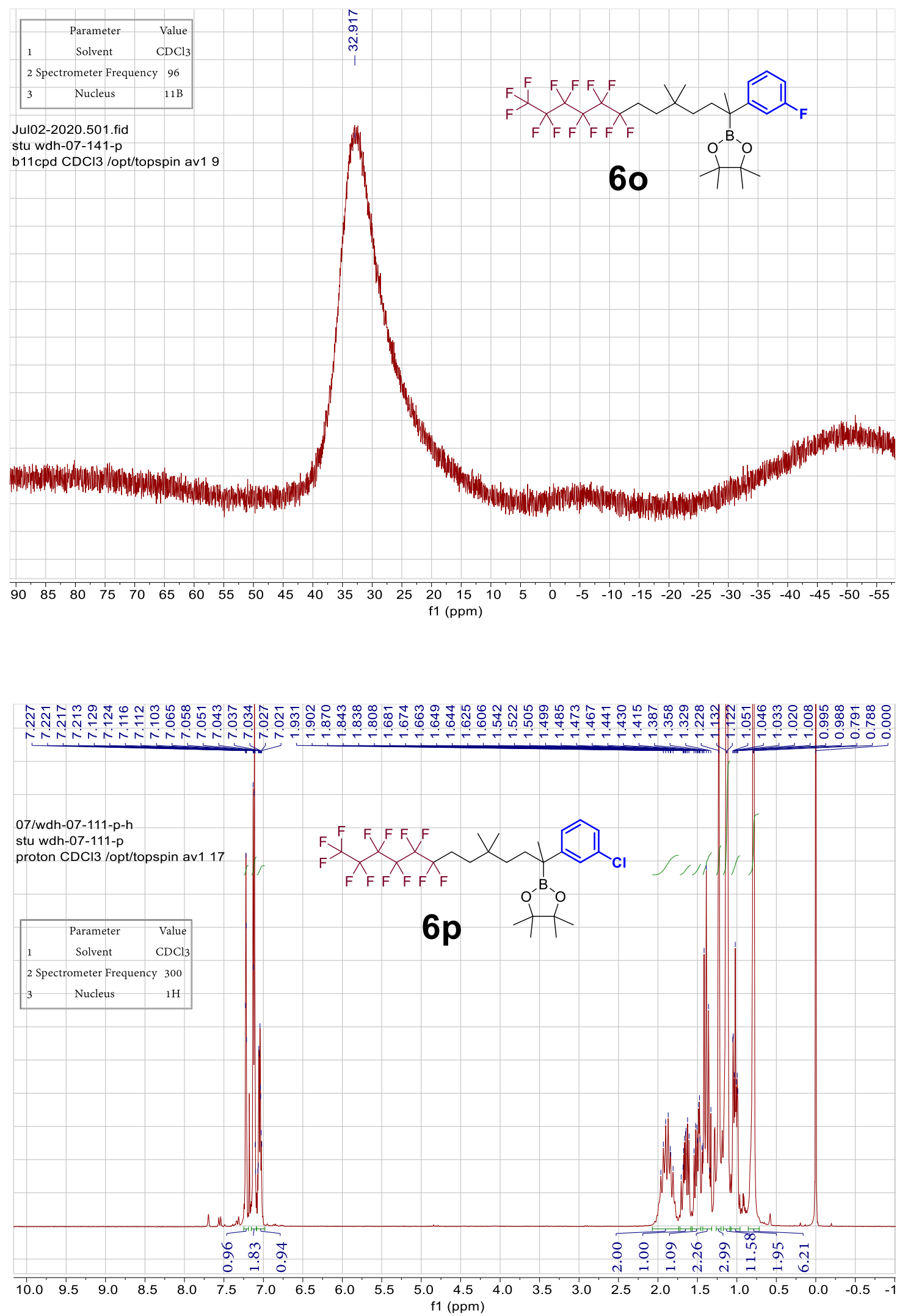

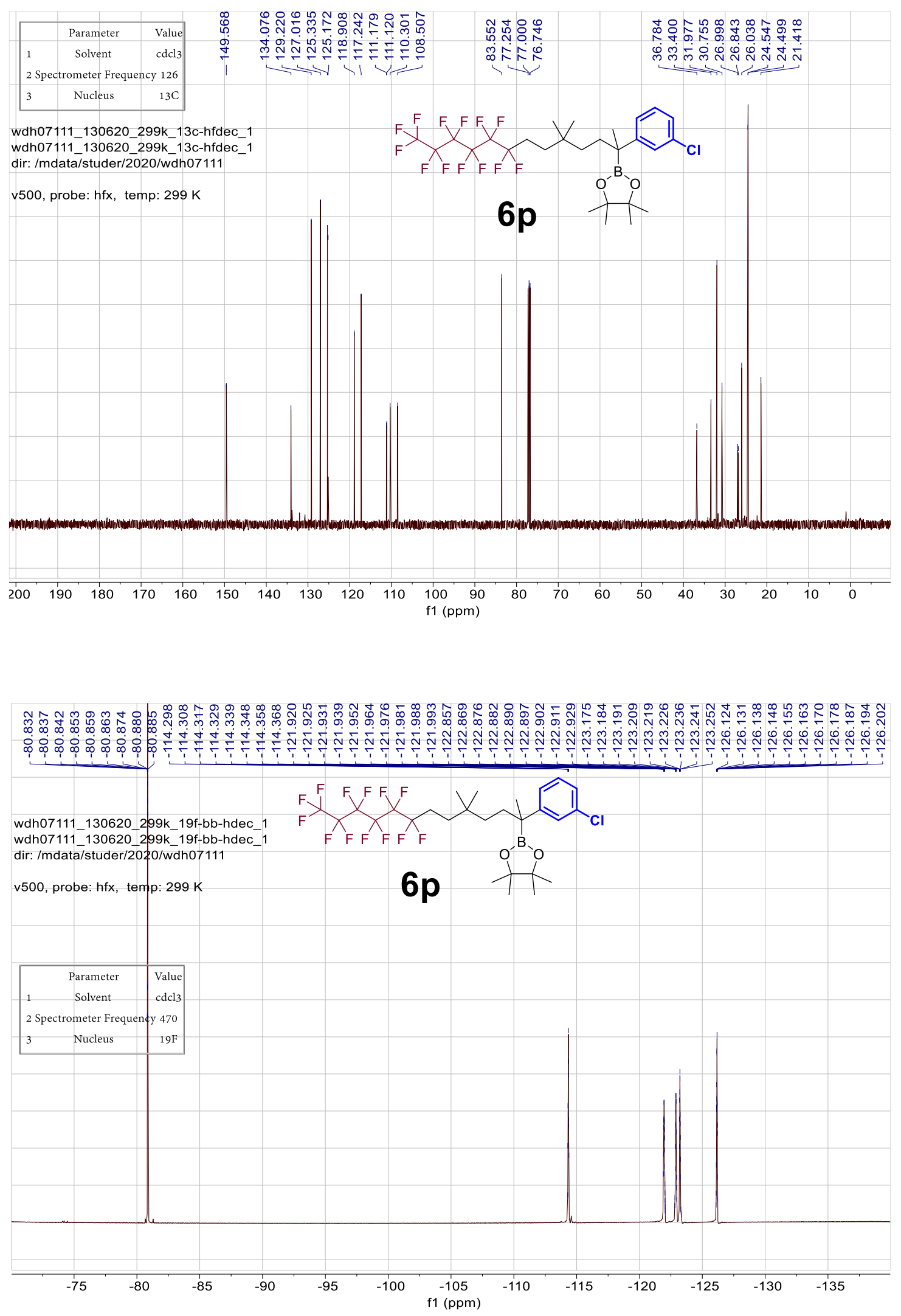

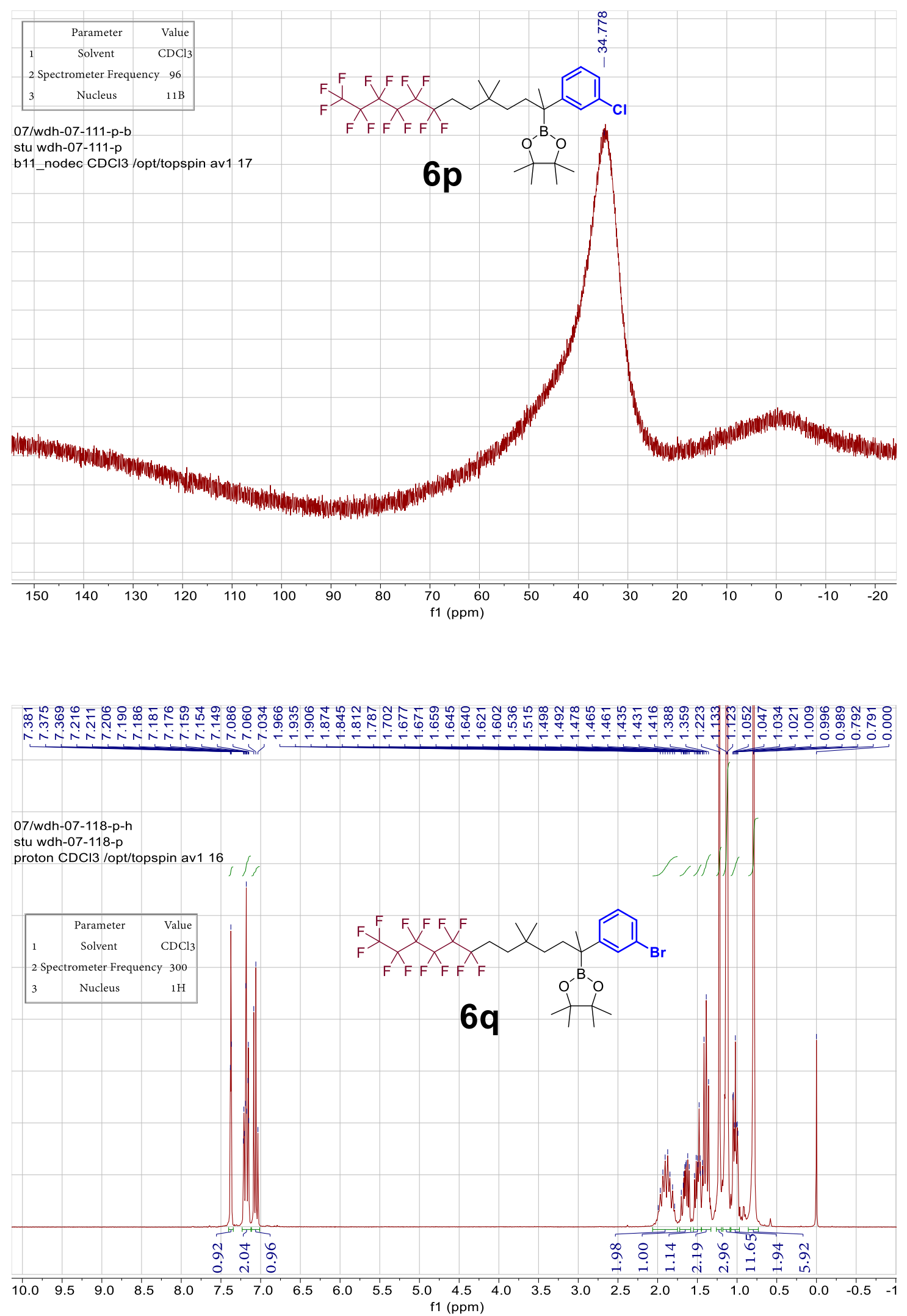

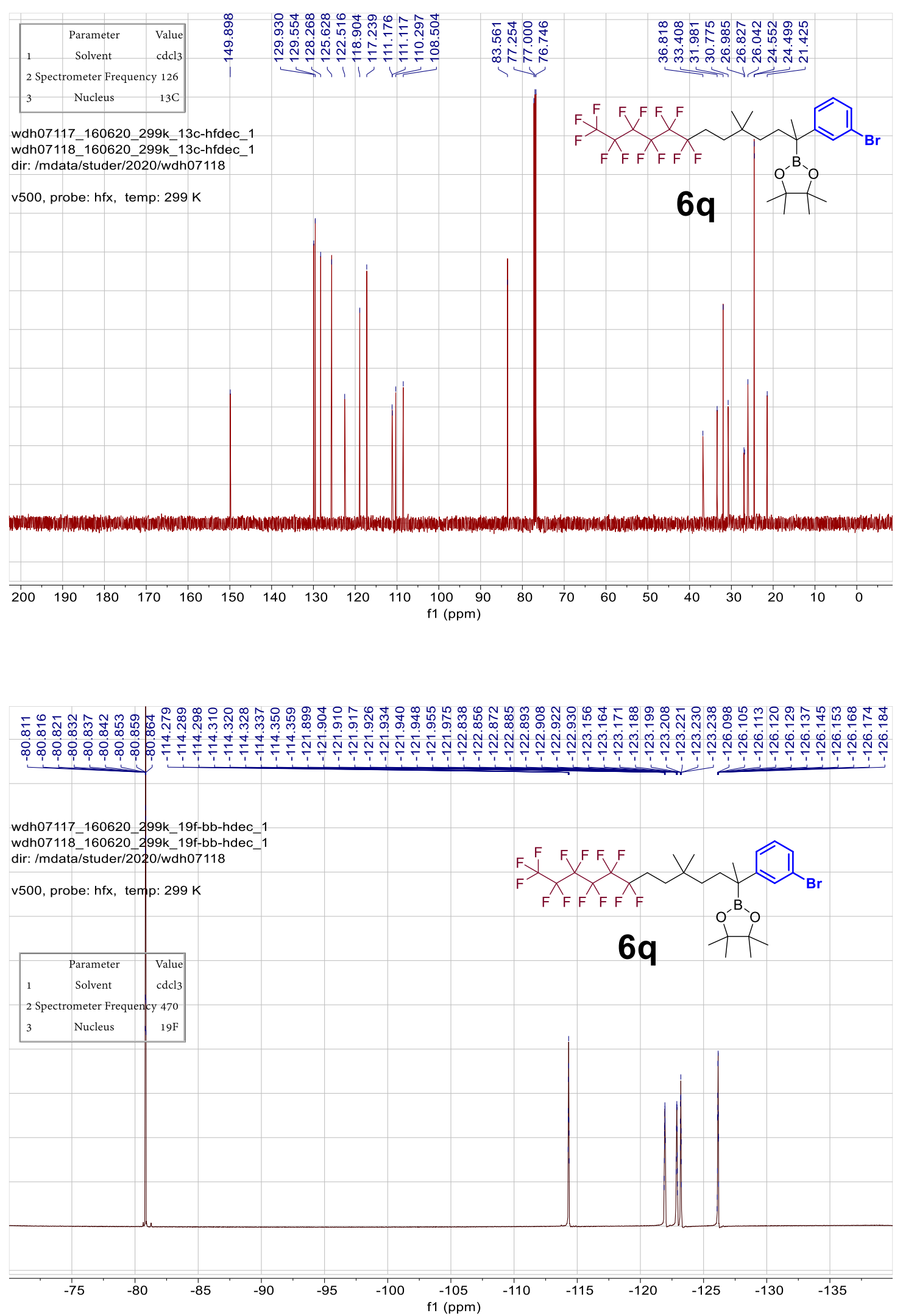

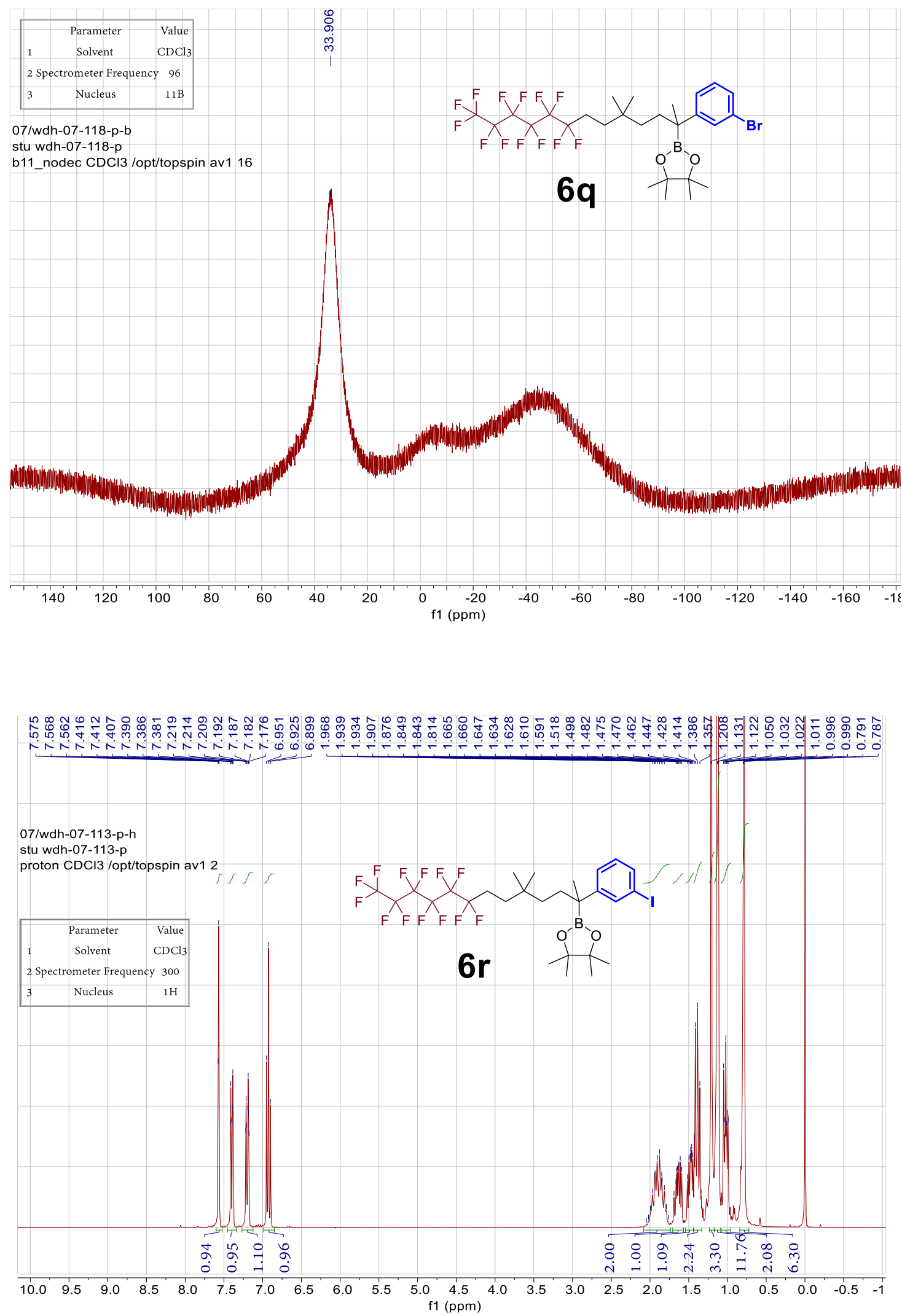


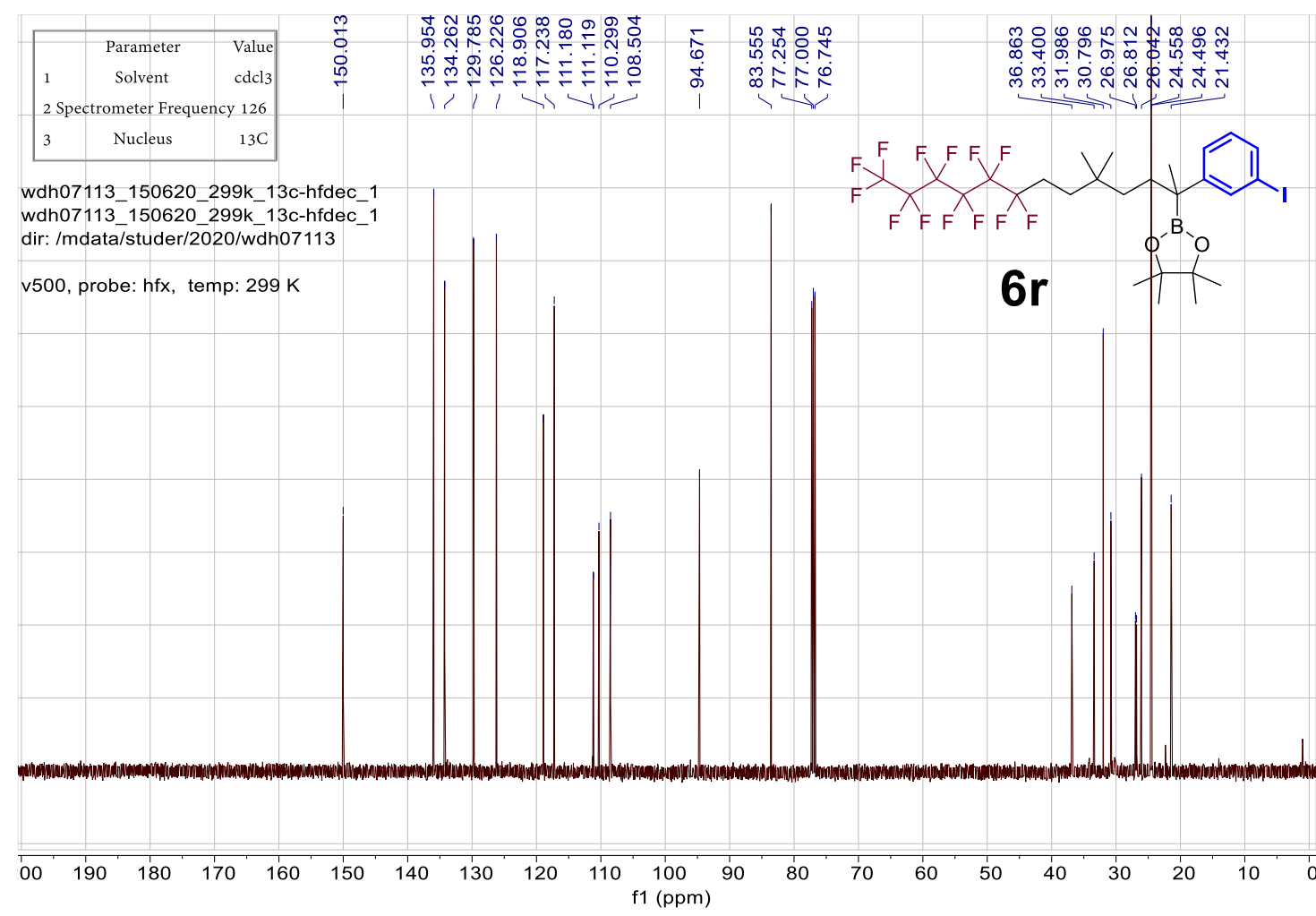

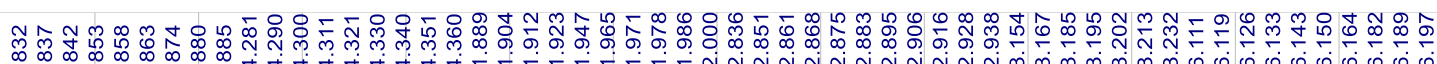

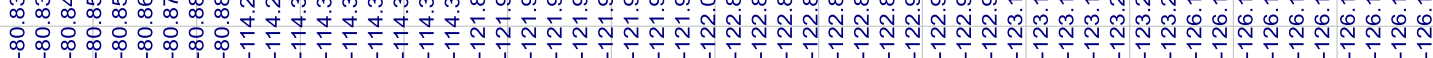

wdh07113_150620_299k_19f-bb-hdec wdh07113_150620_299k_19f-bb-hdec_1

dir: /mdata/studer/2020/wdh07113

v500, probe: hfx, temp: $299 \mathrm{~K}$
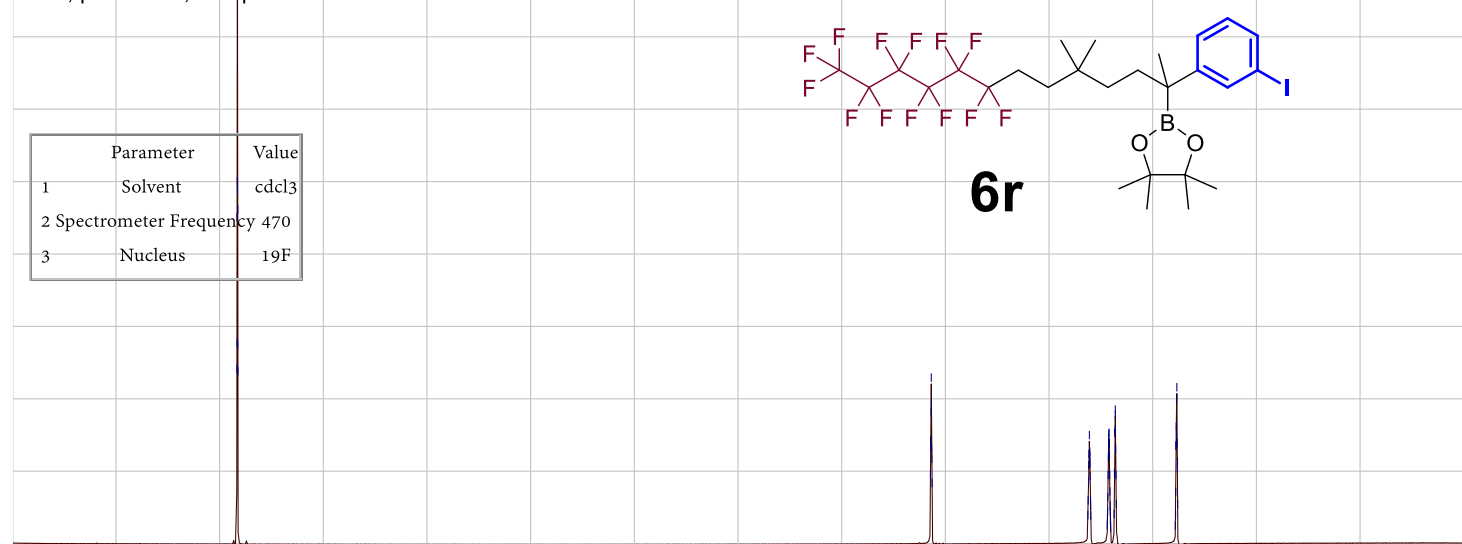

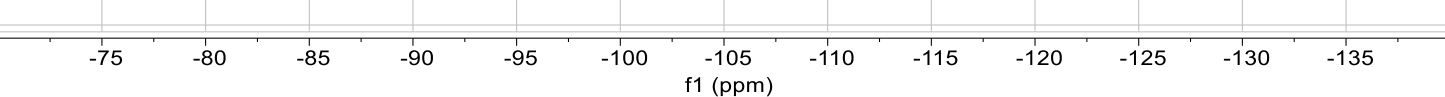



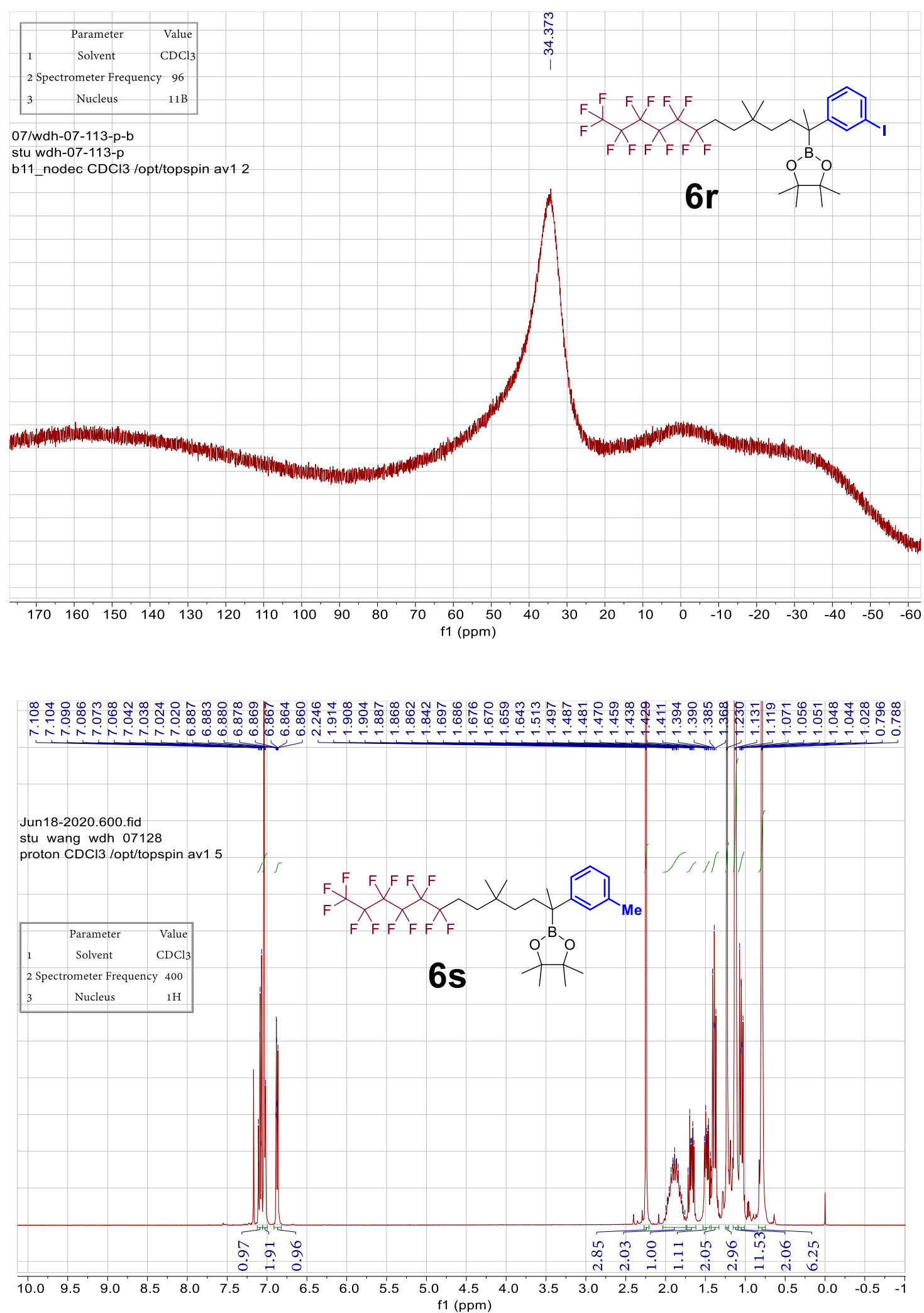

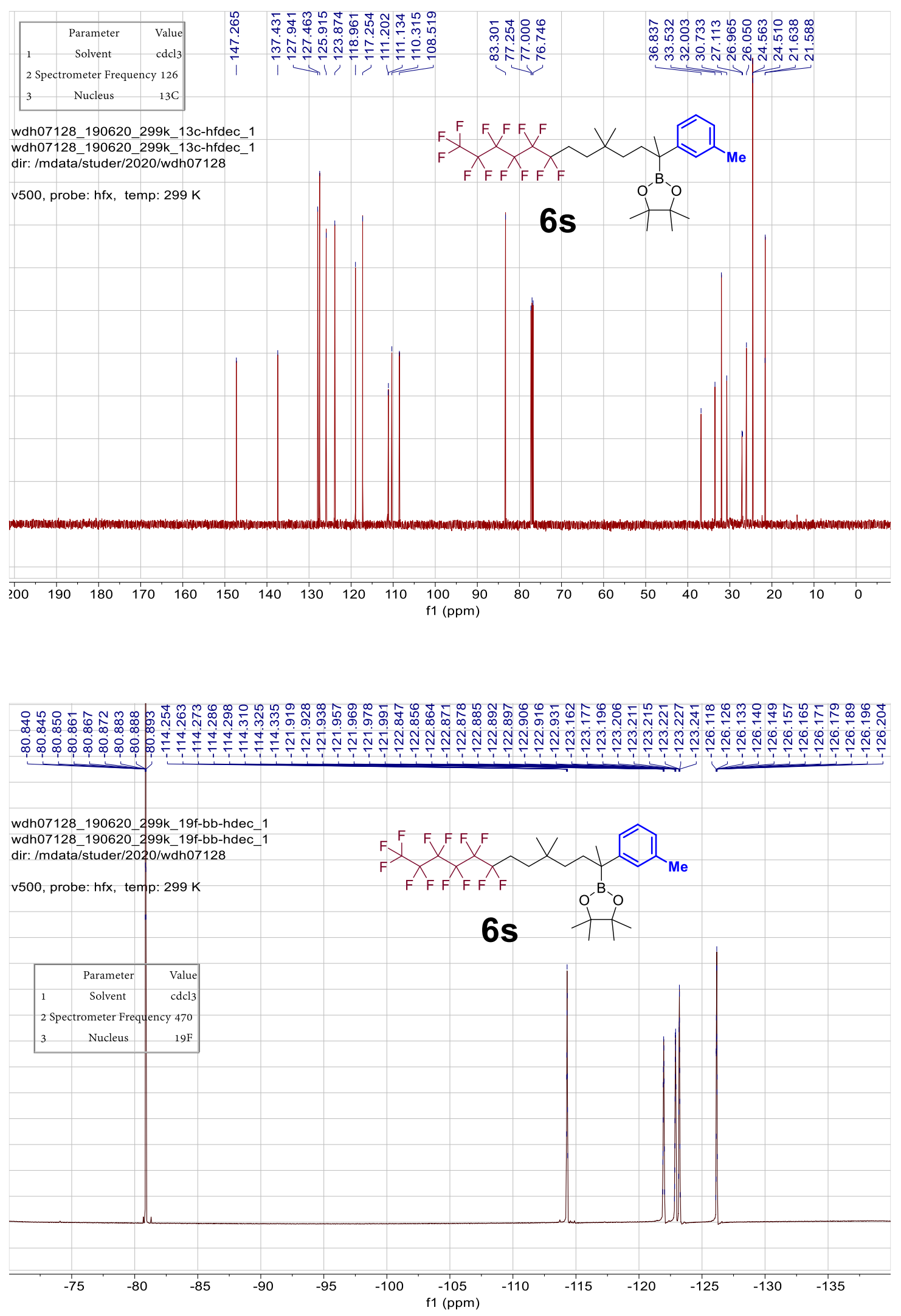


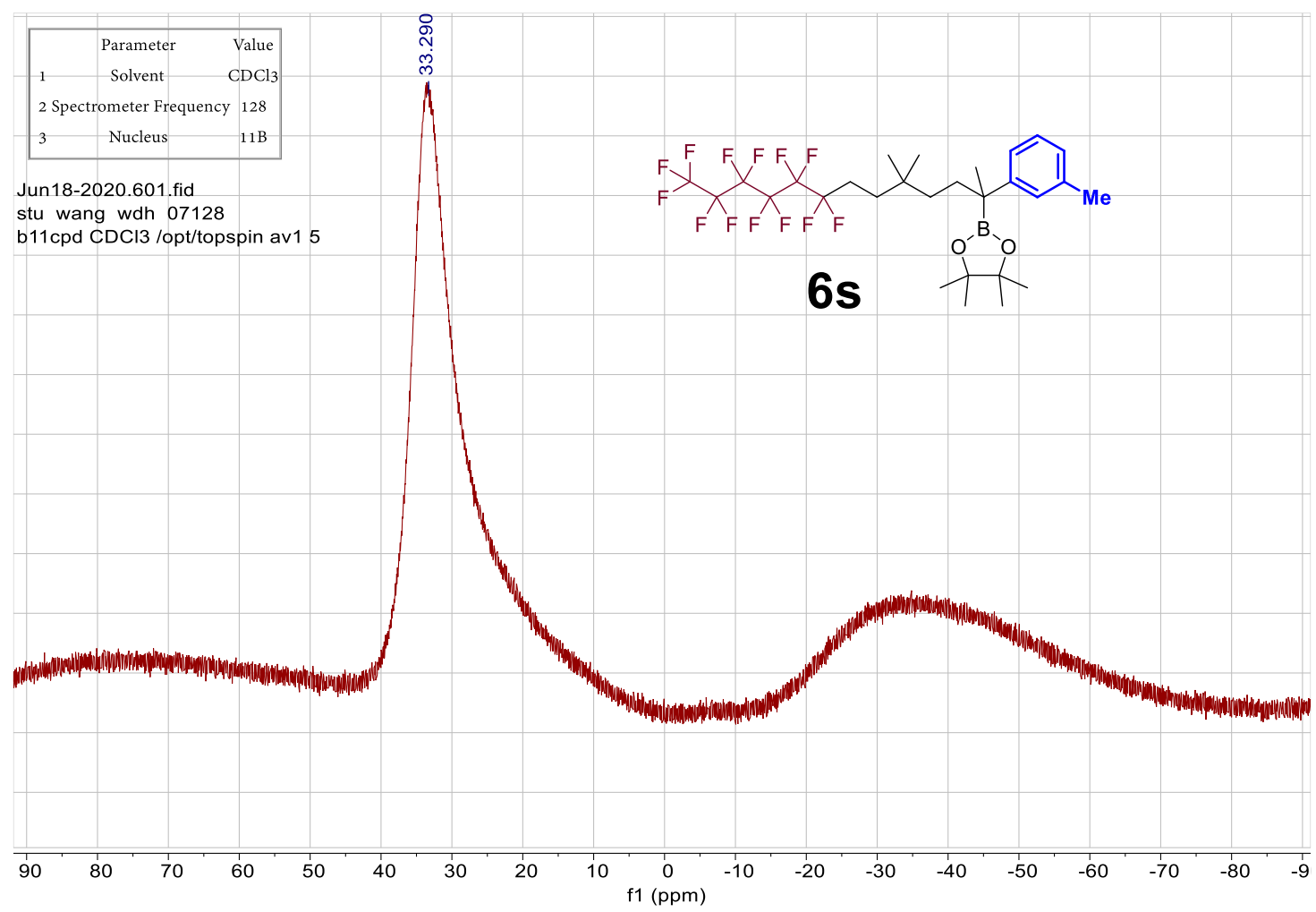

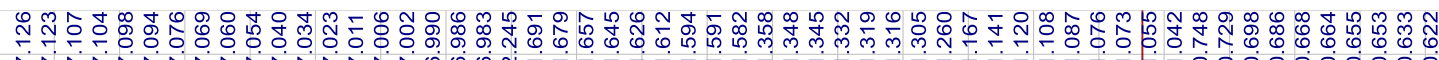

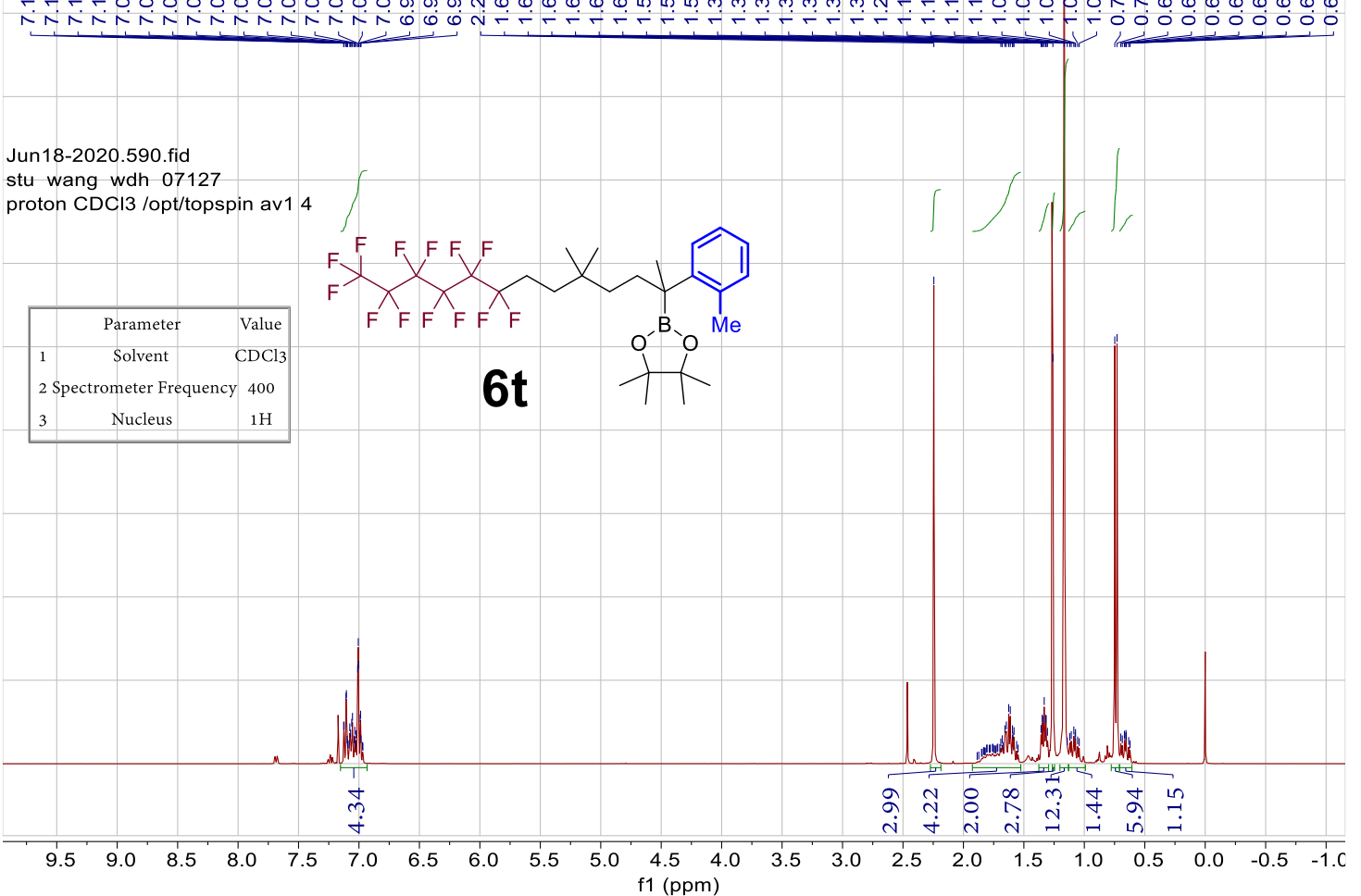



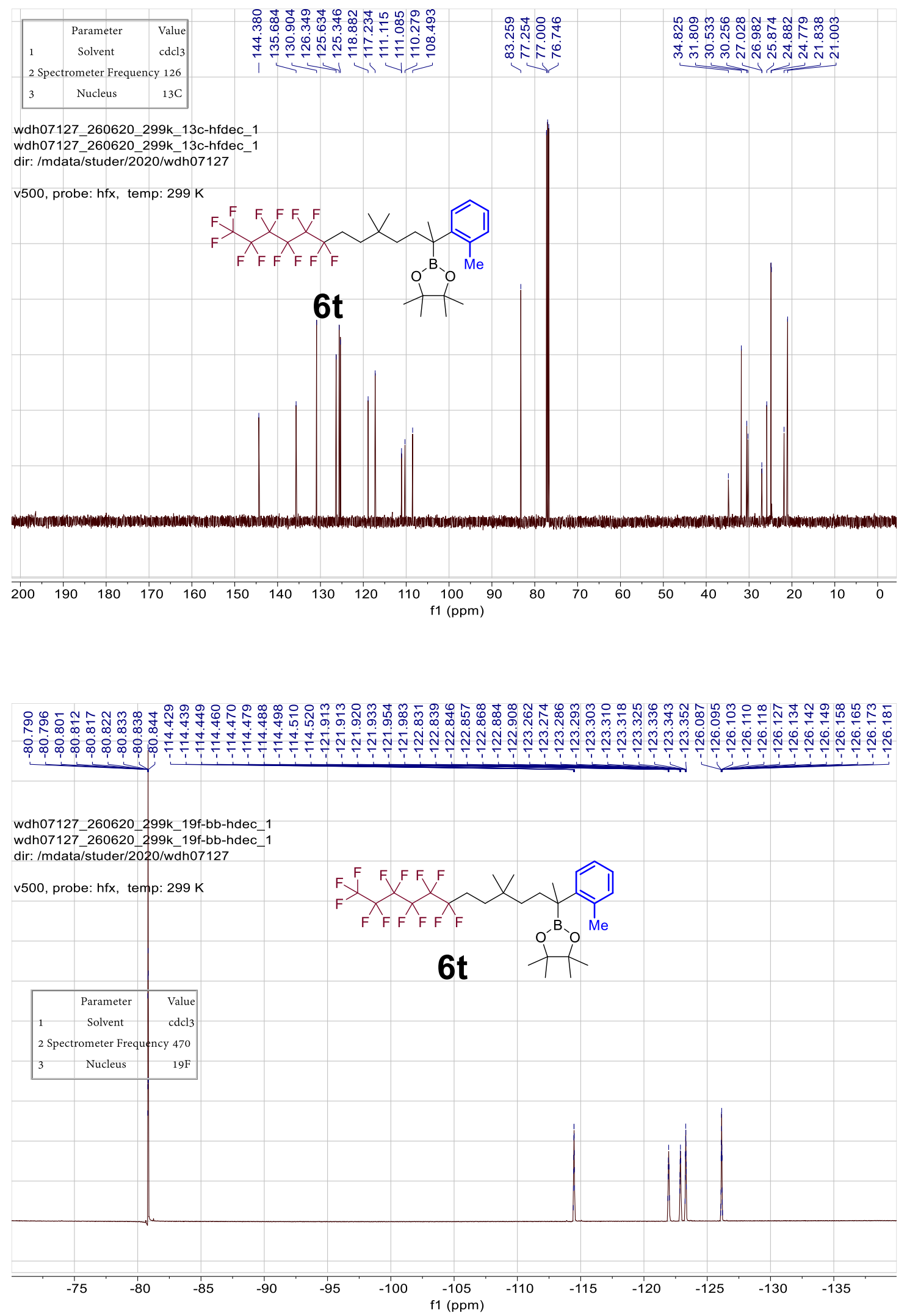

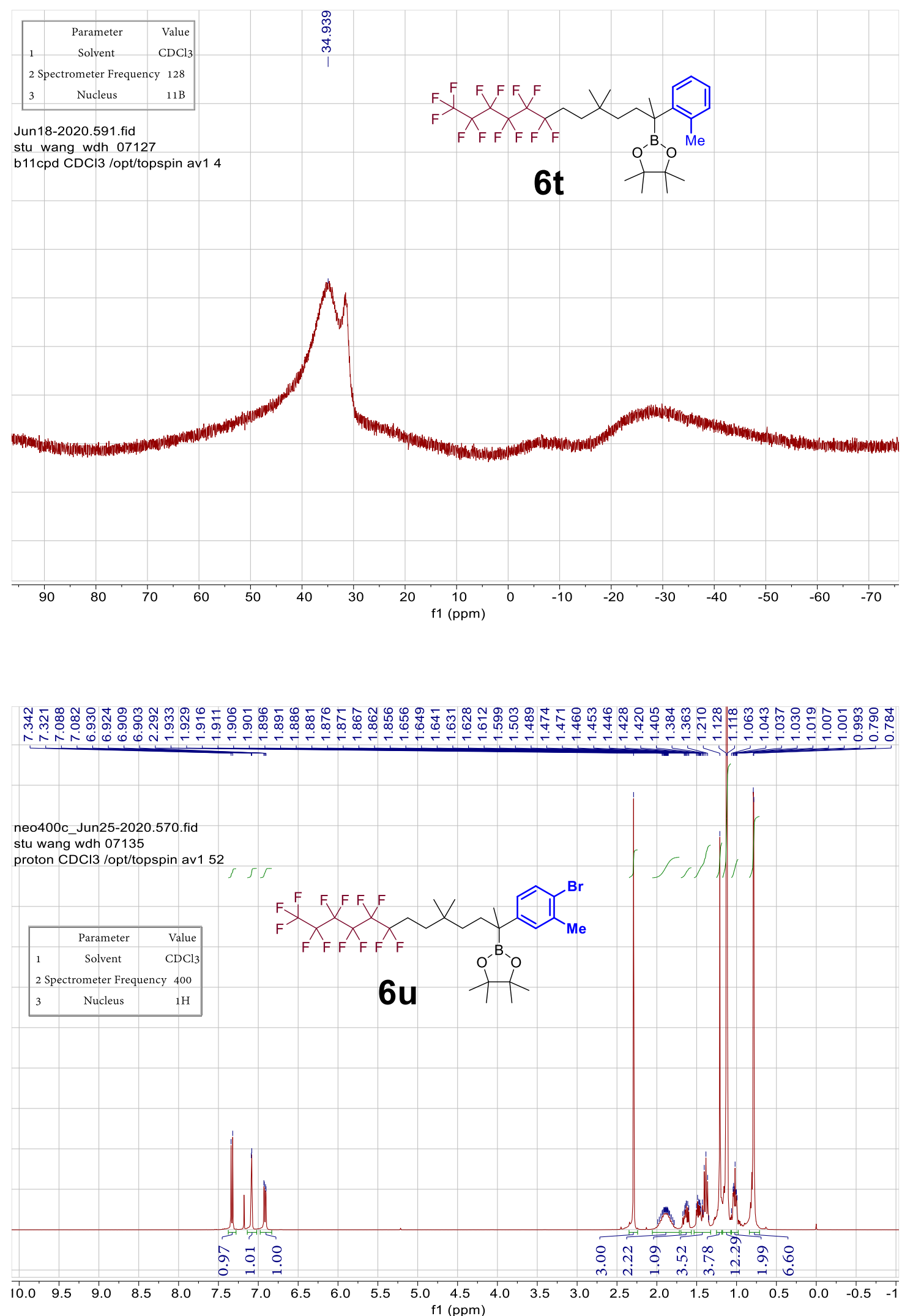

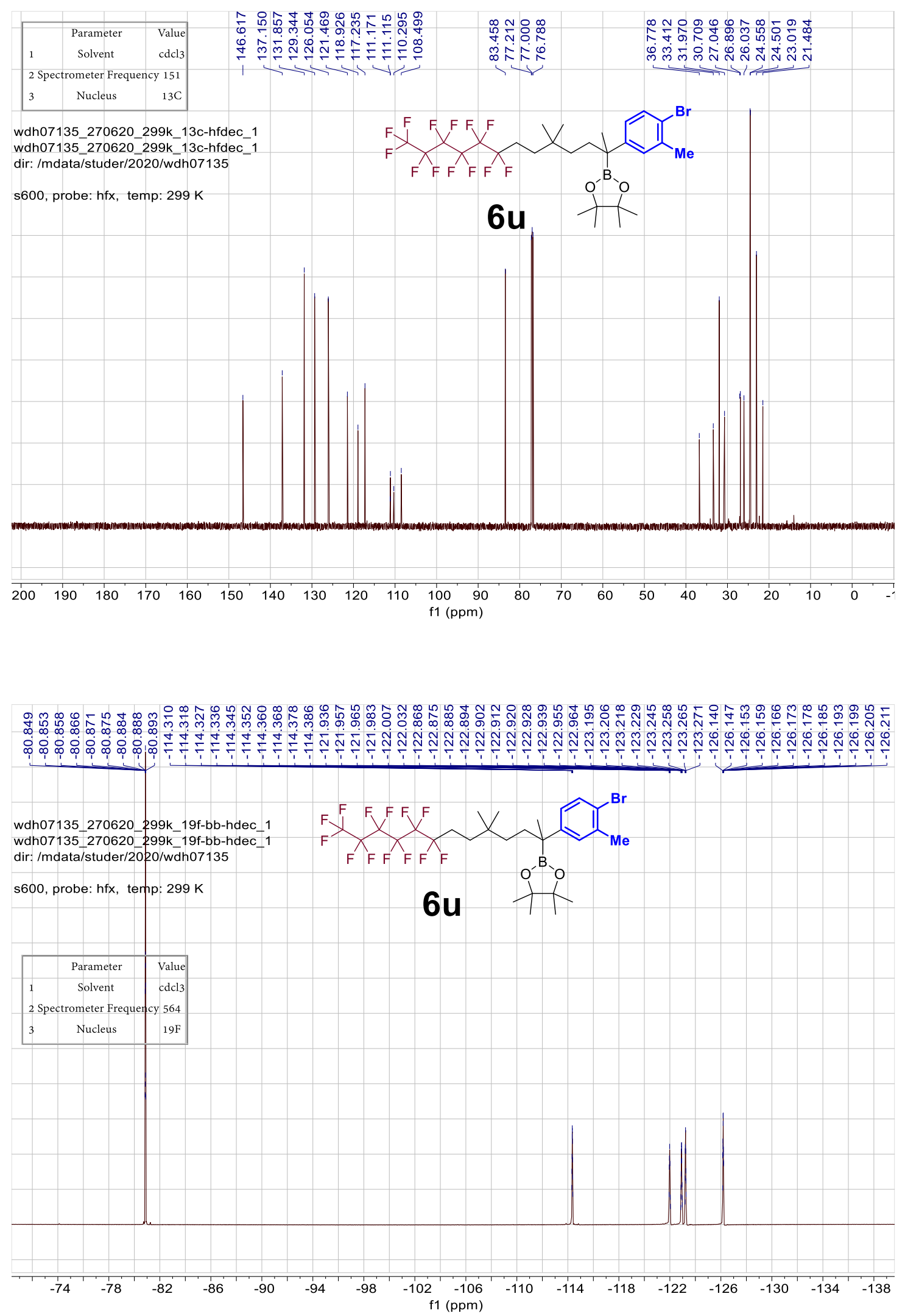

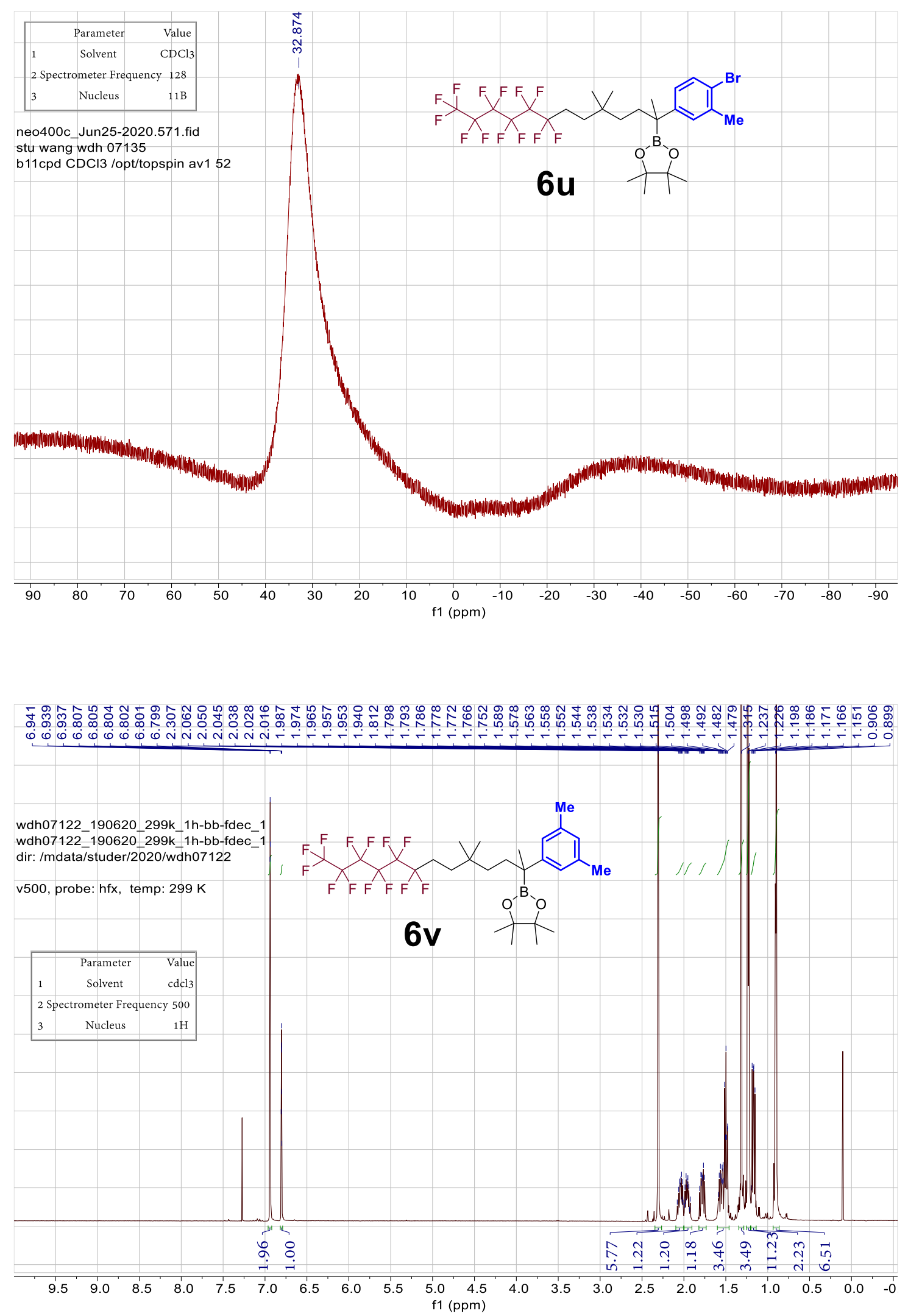

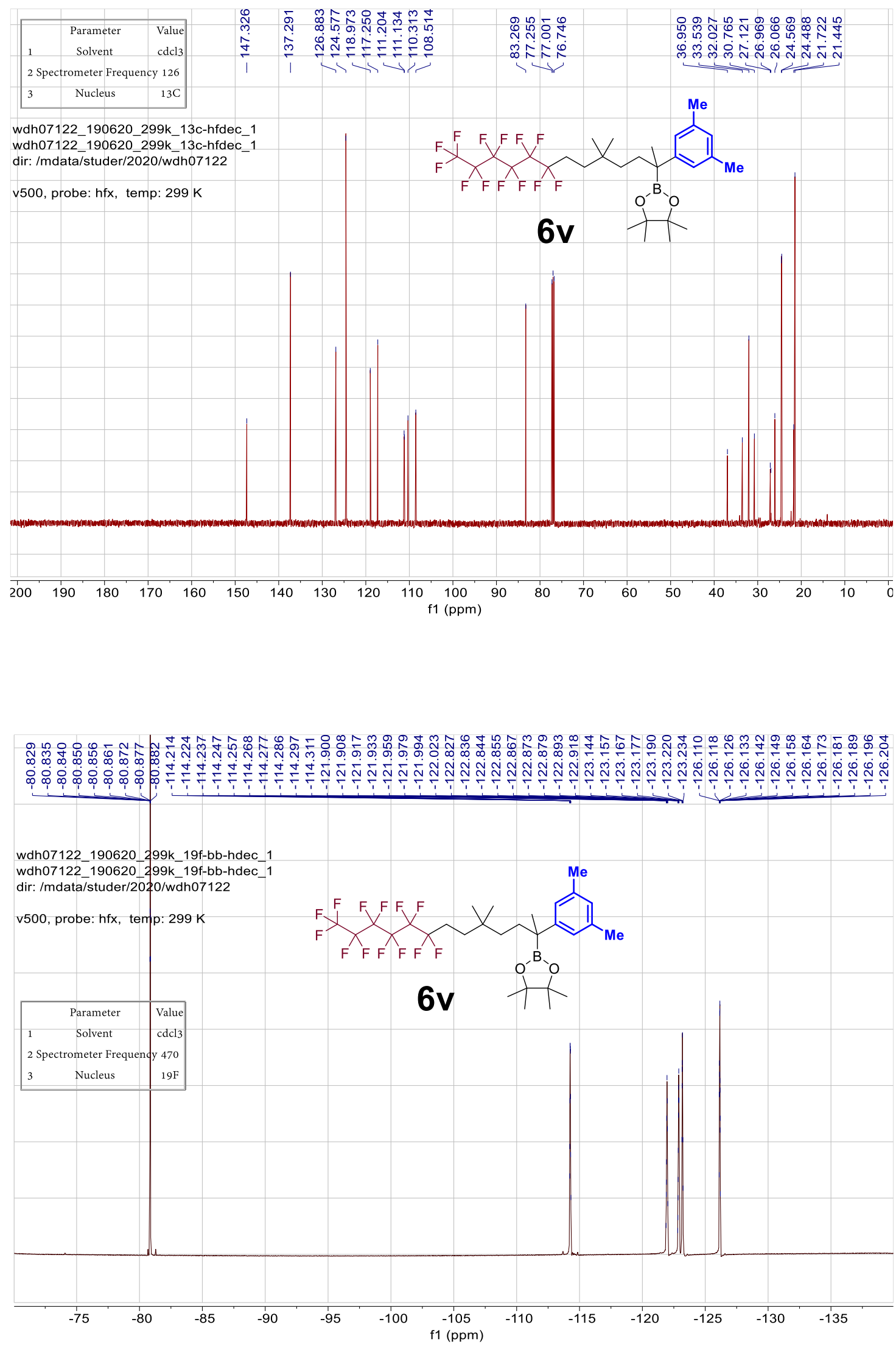

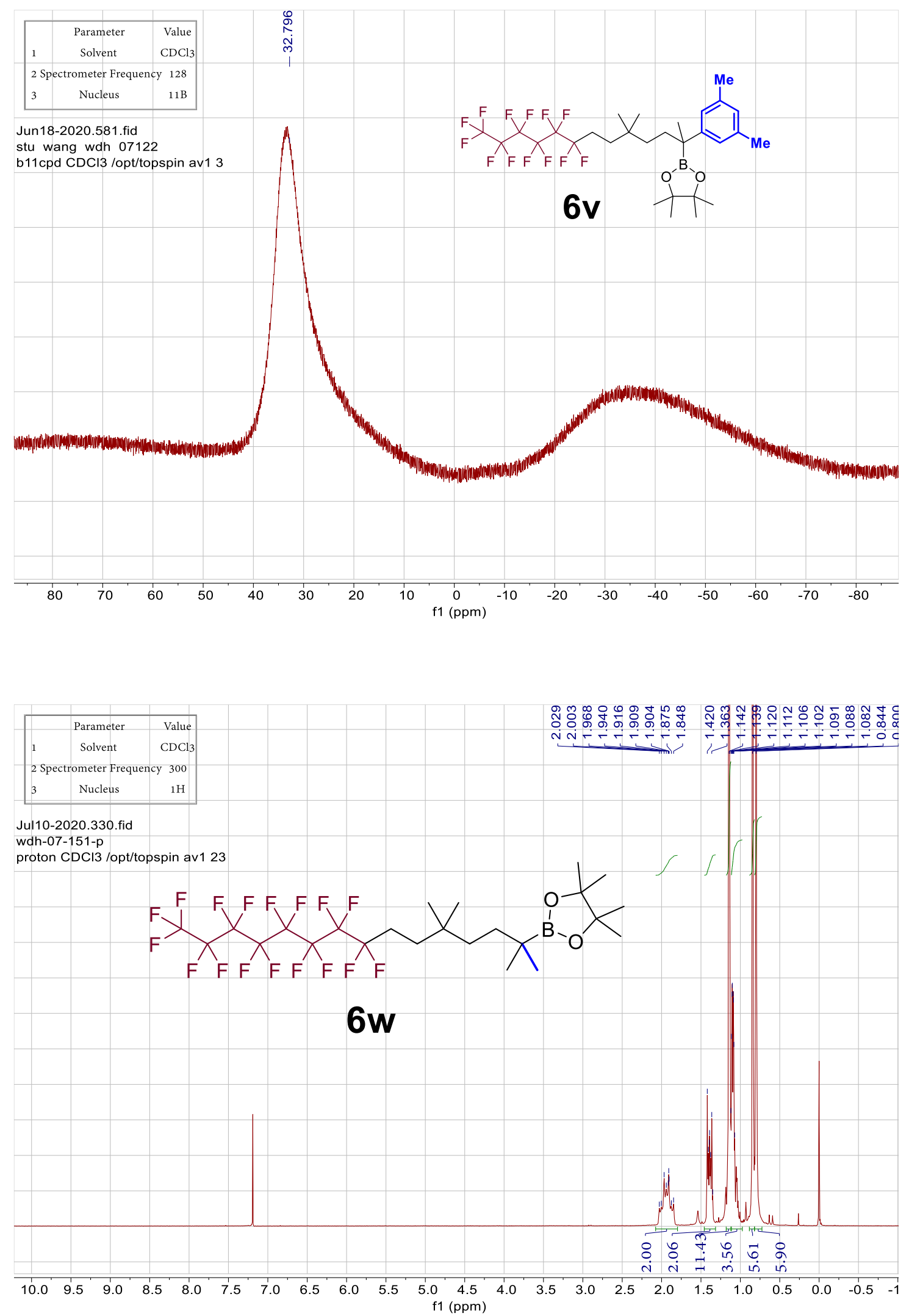

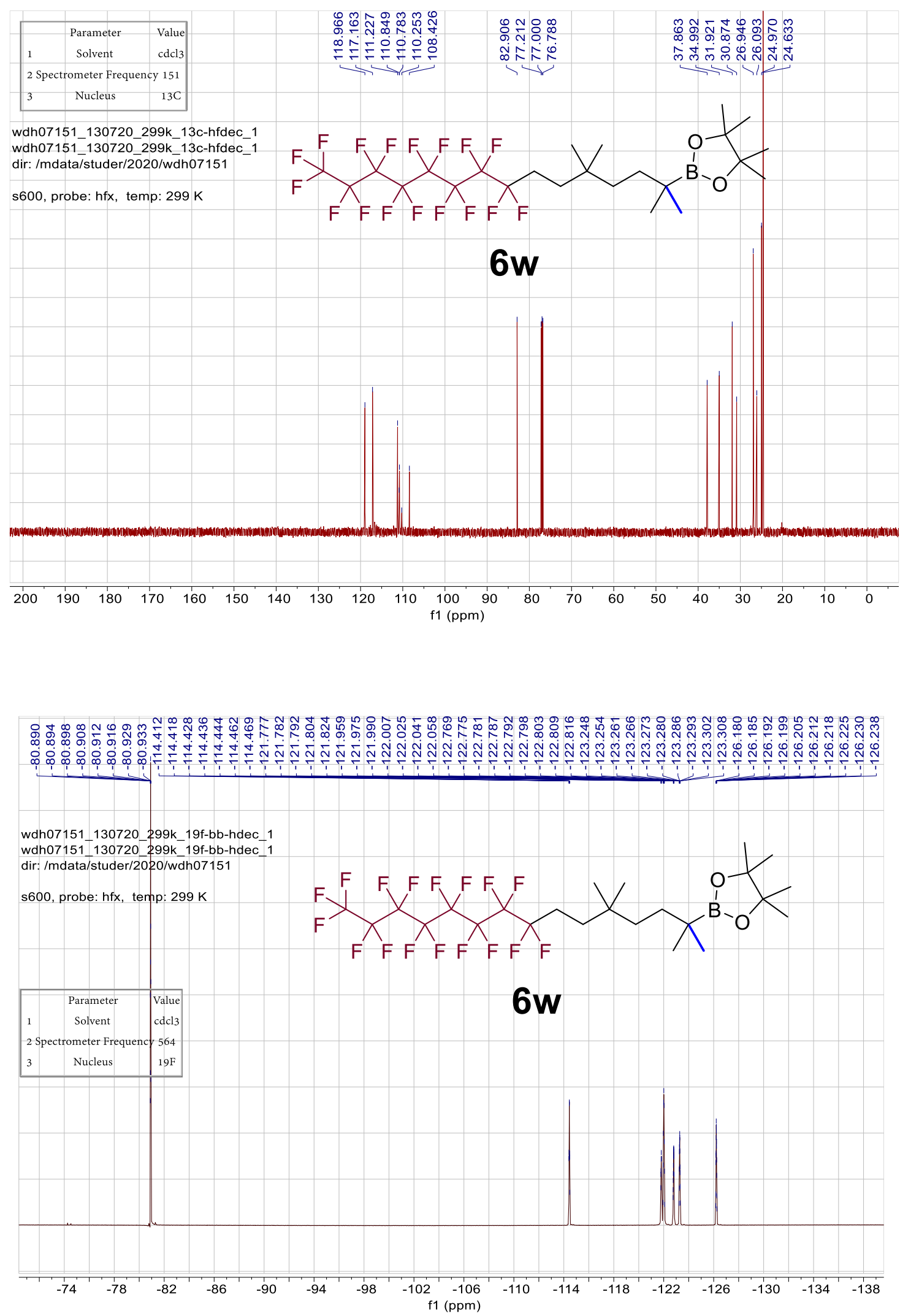

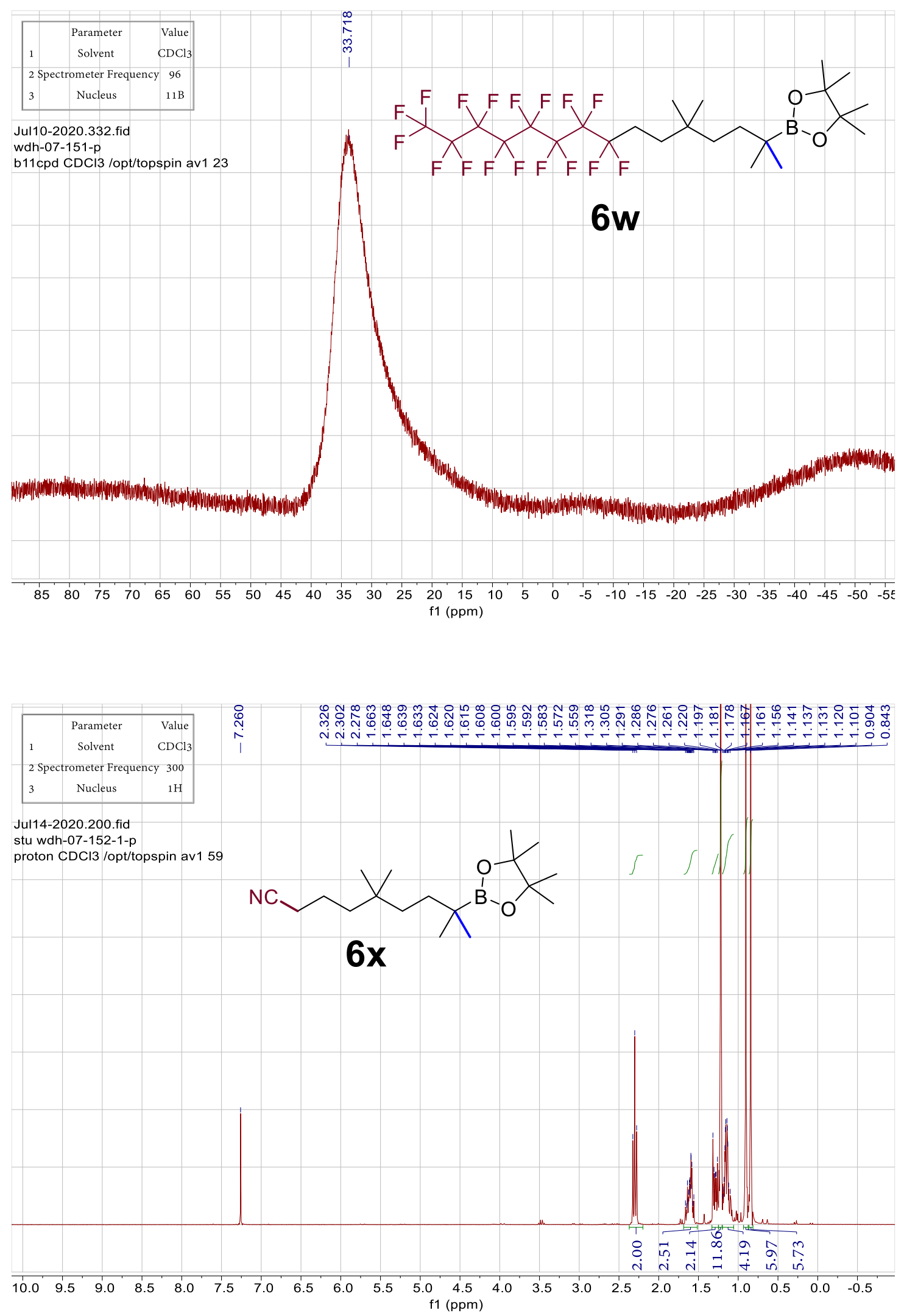

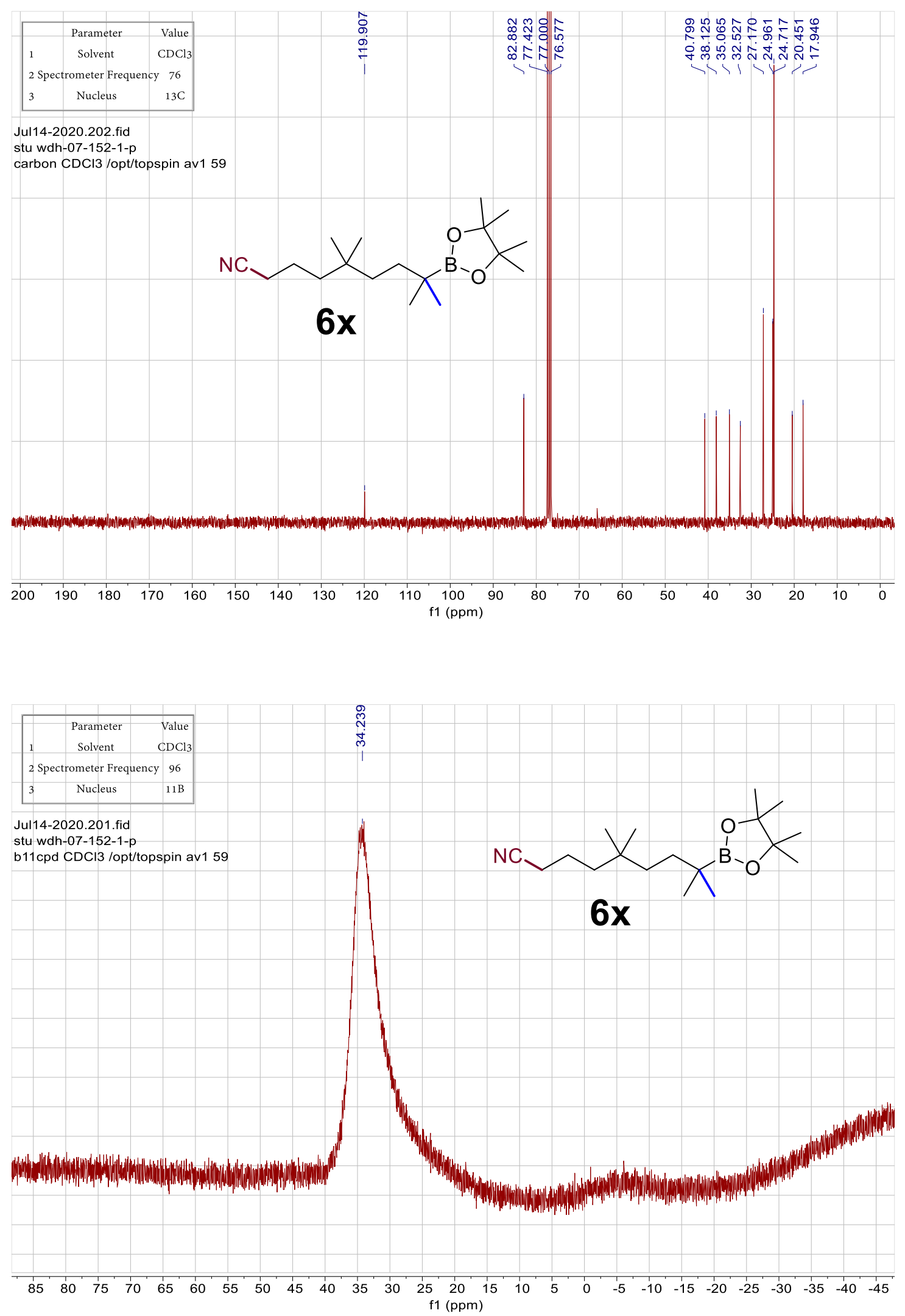

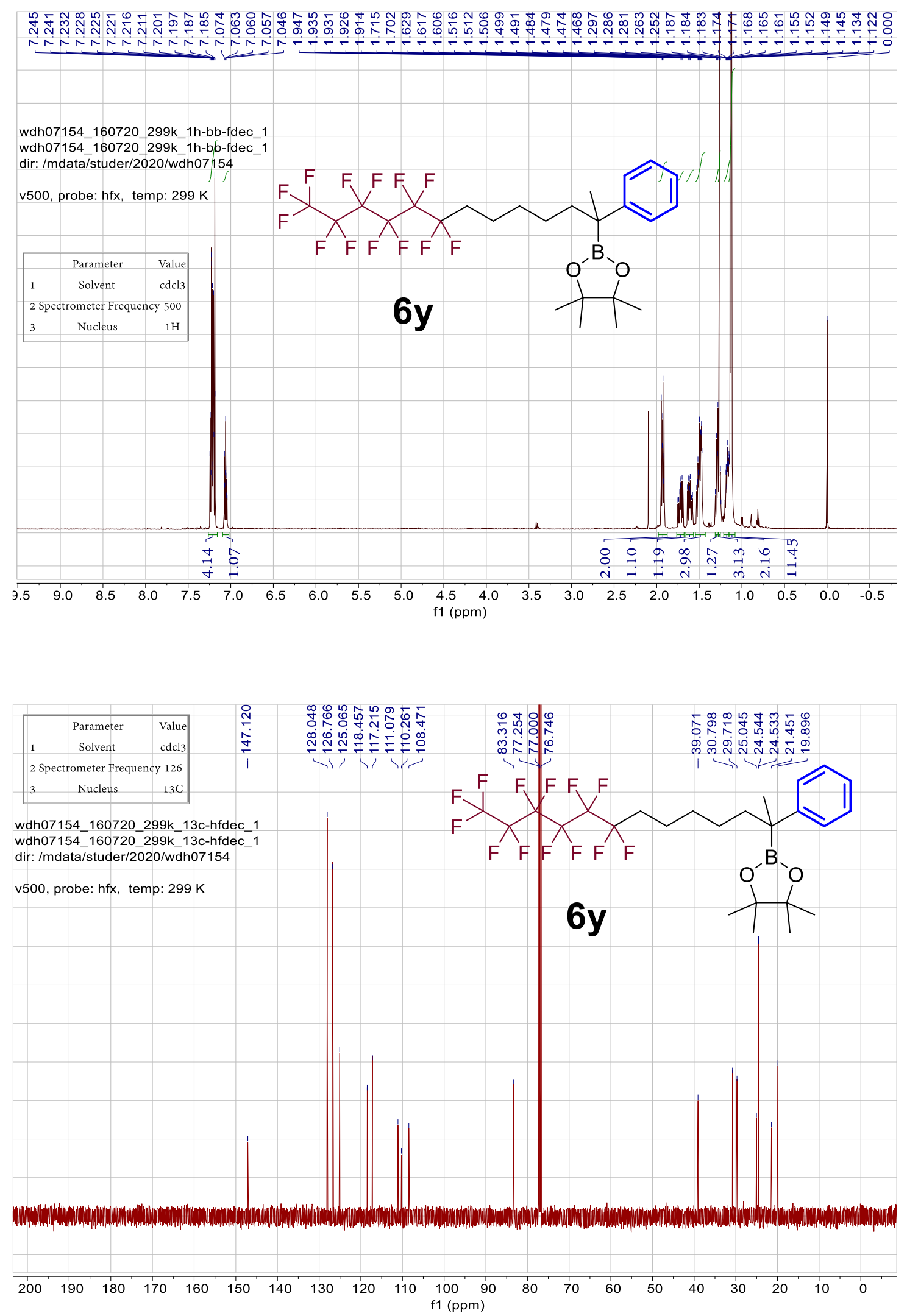


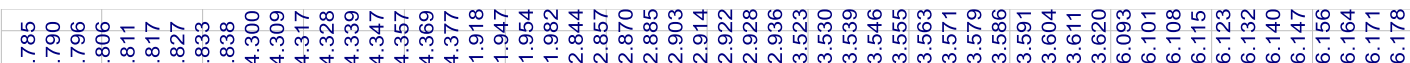
o.
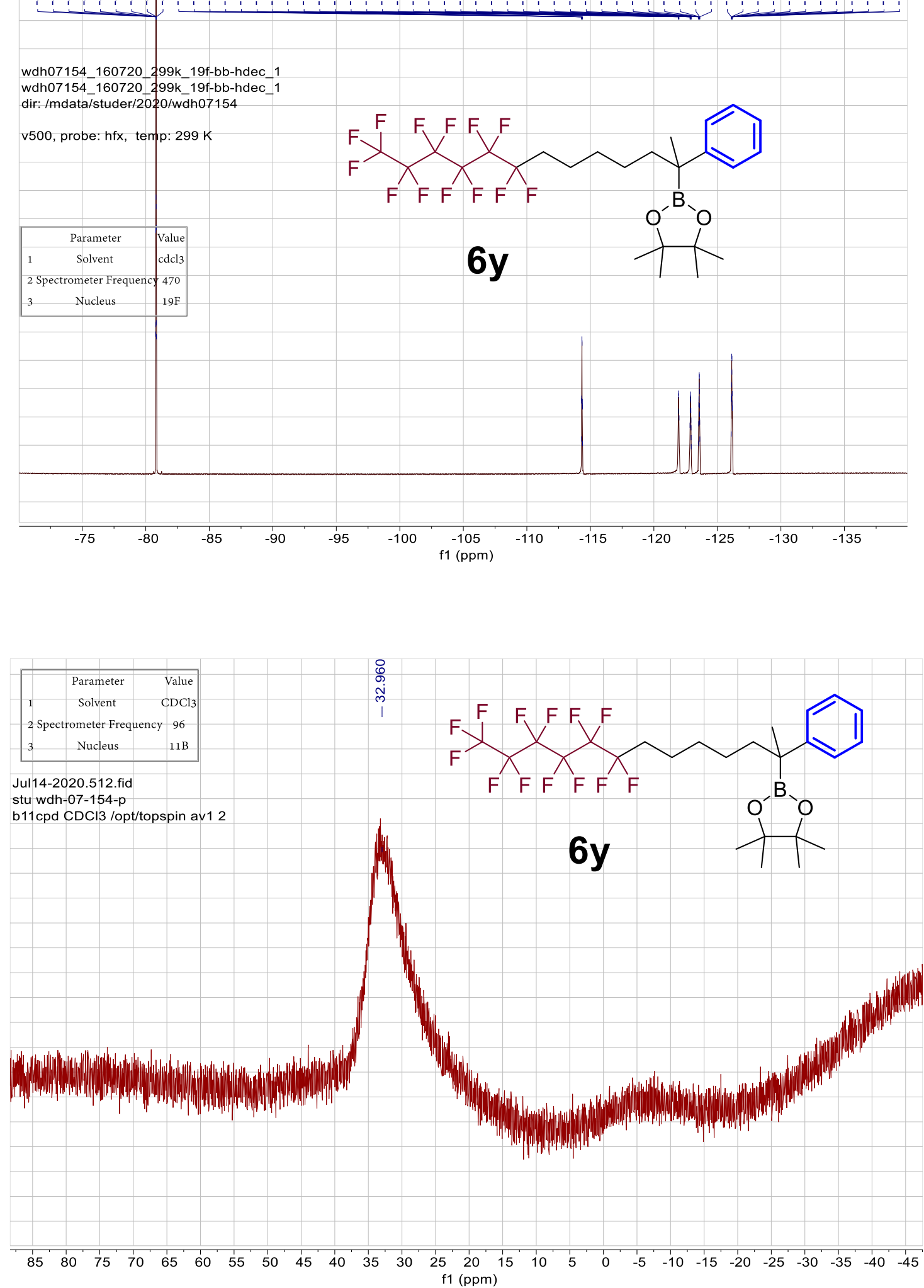

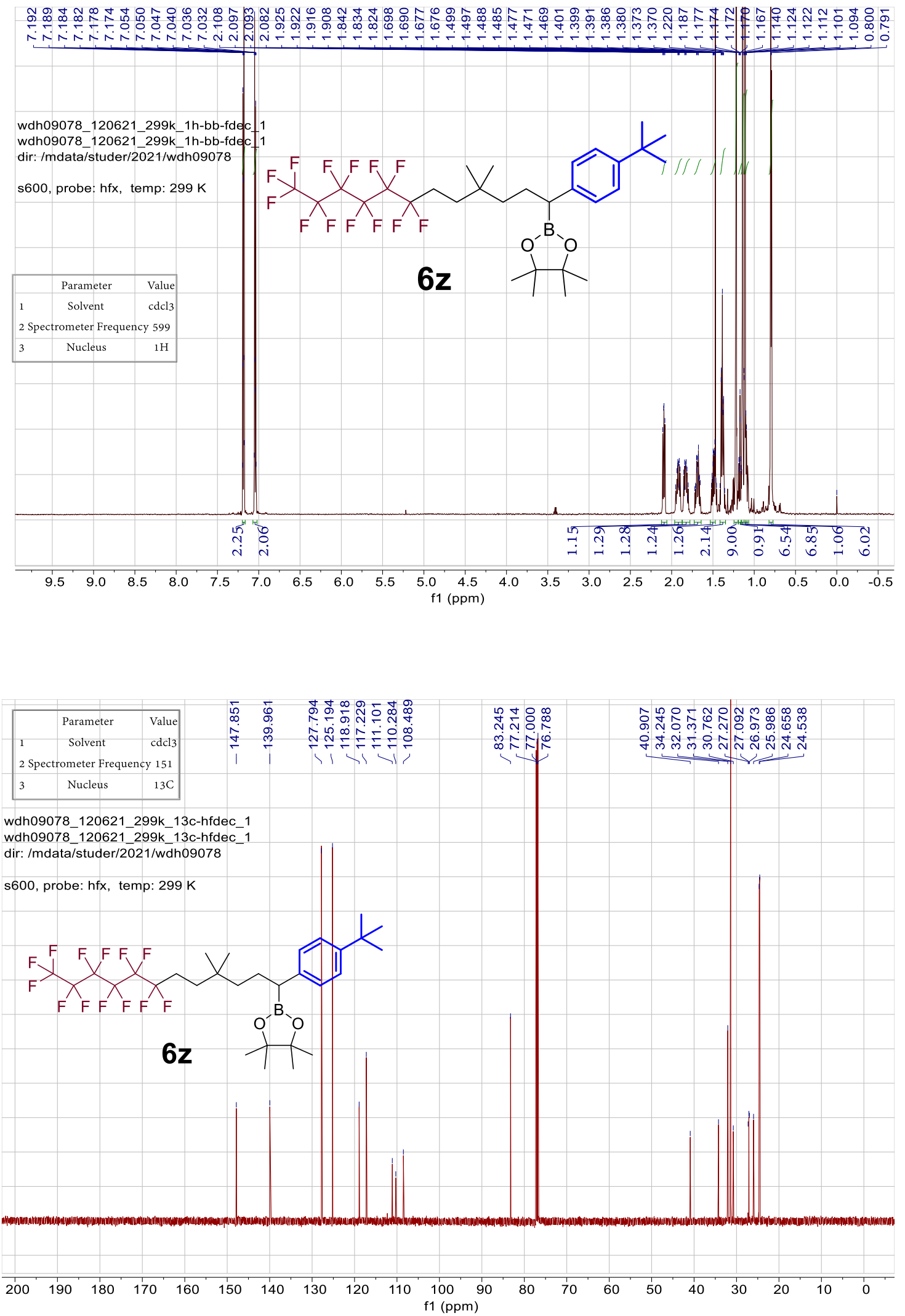

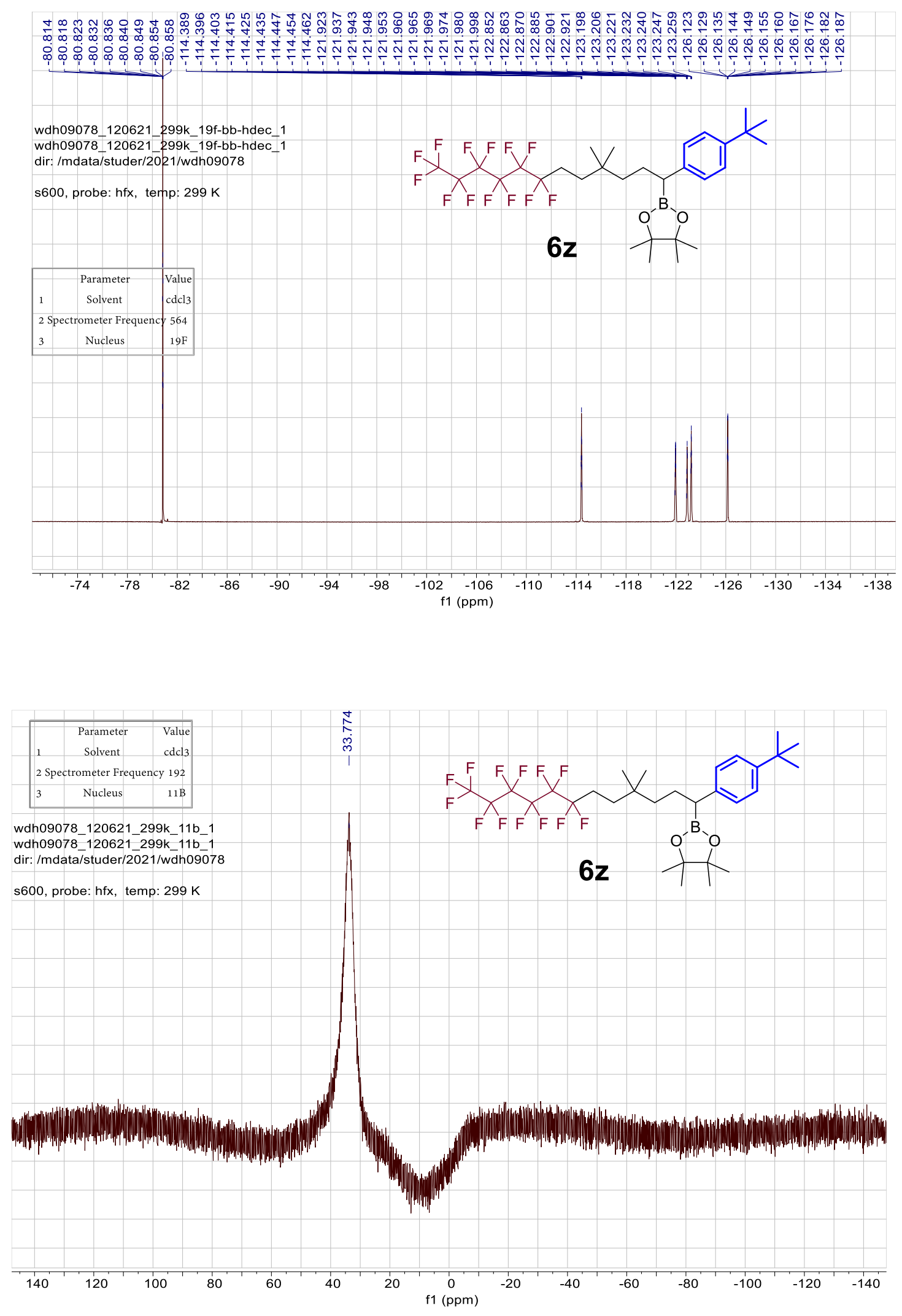

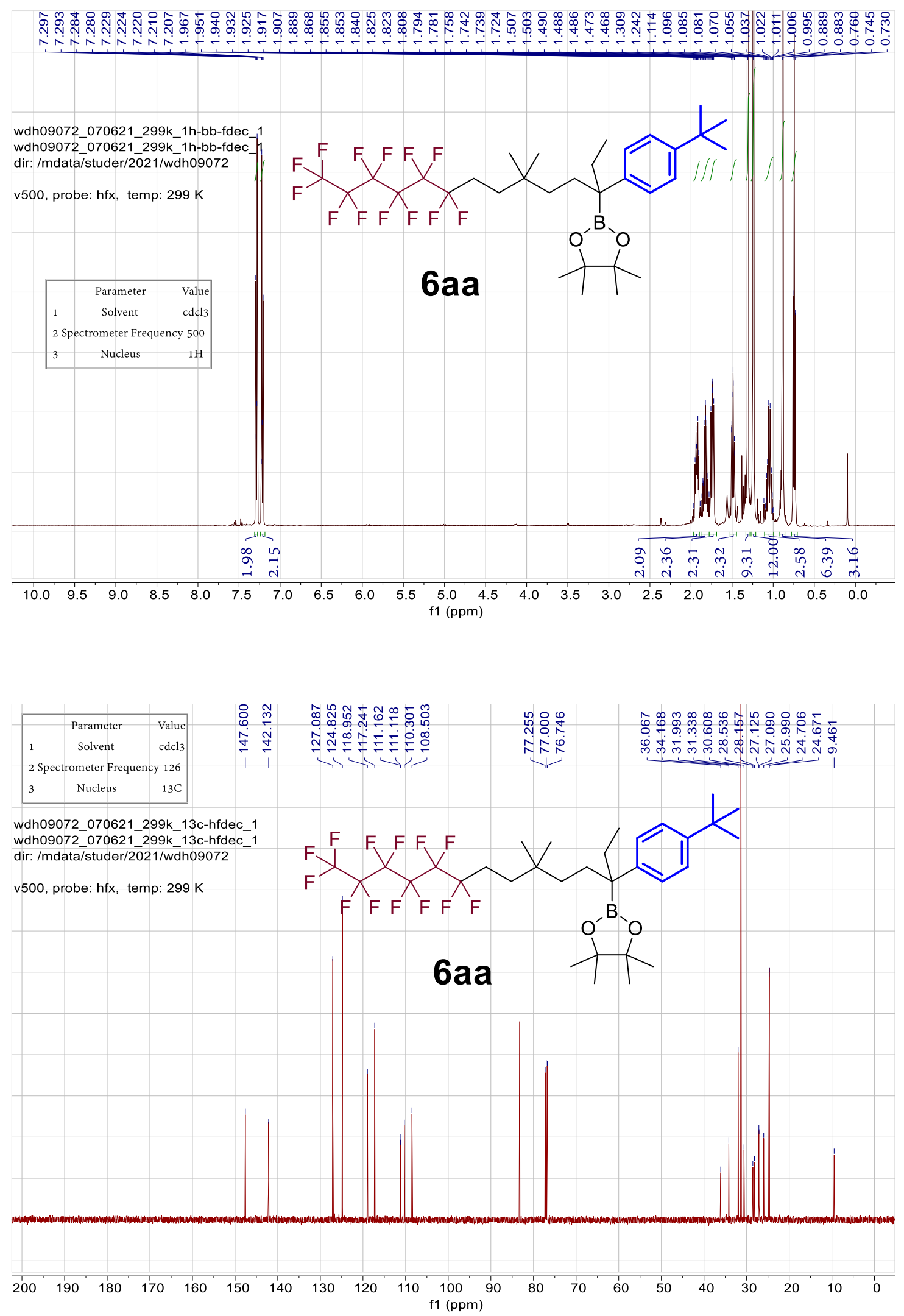

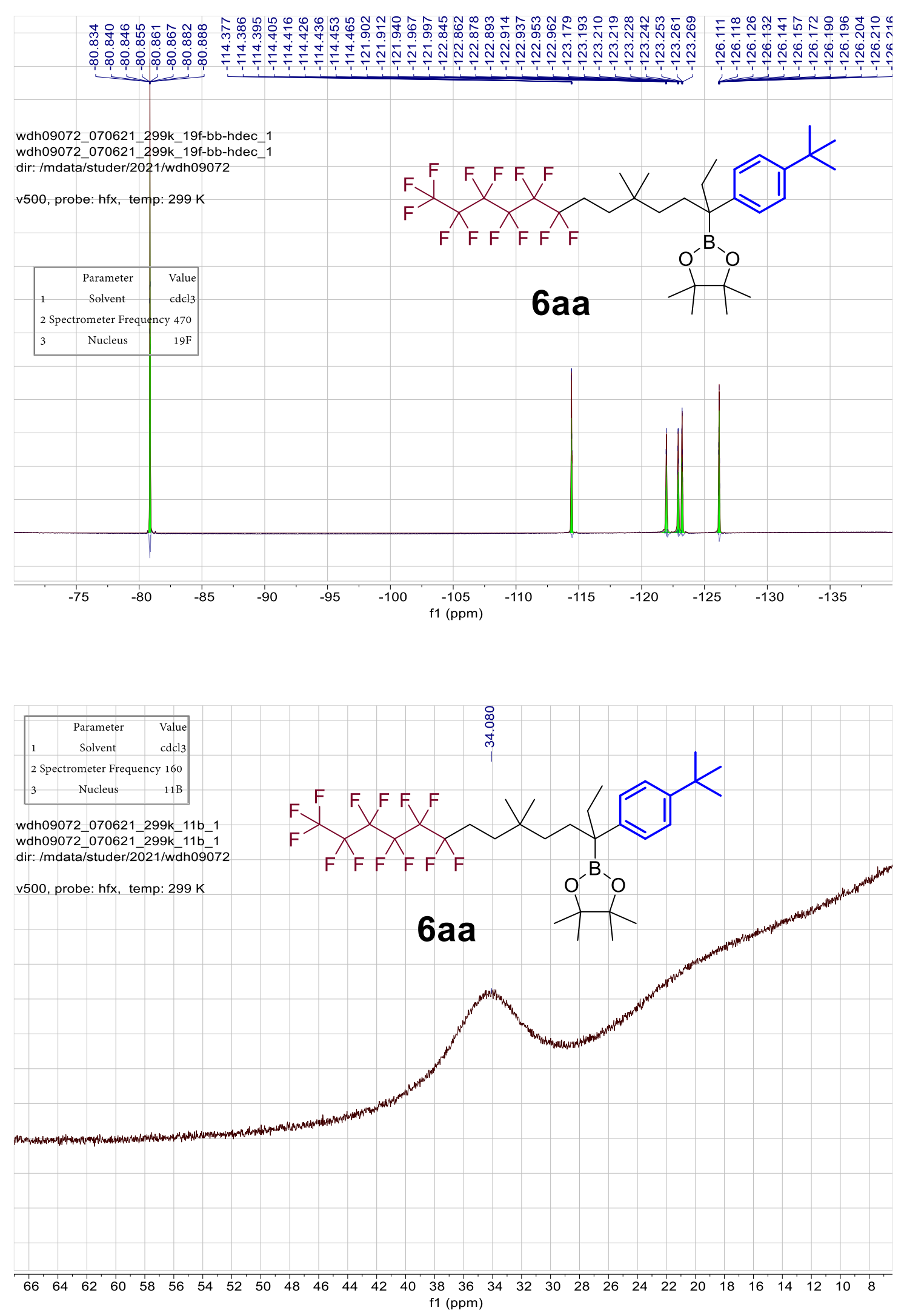

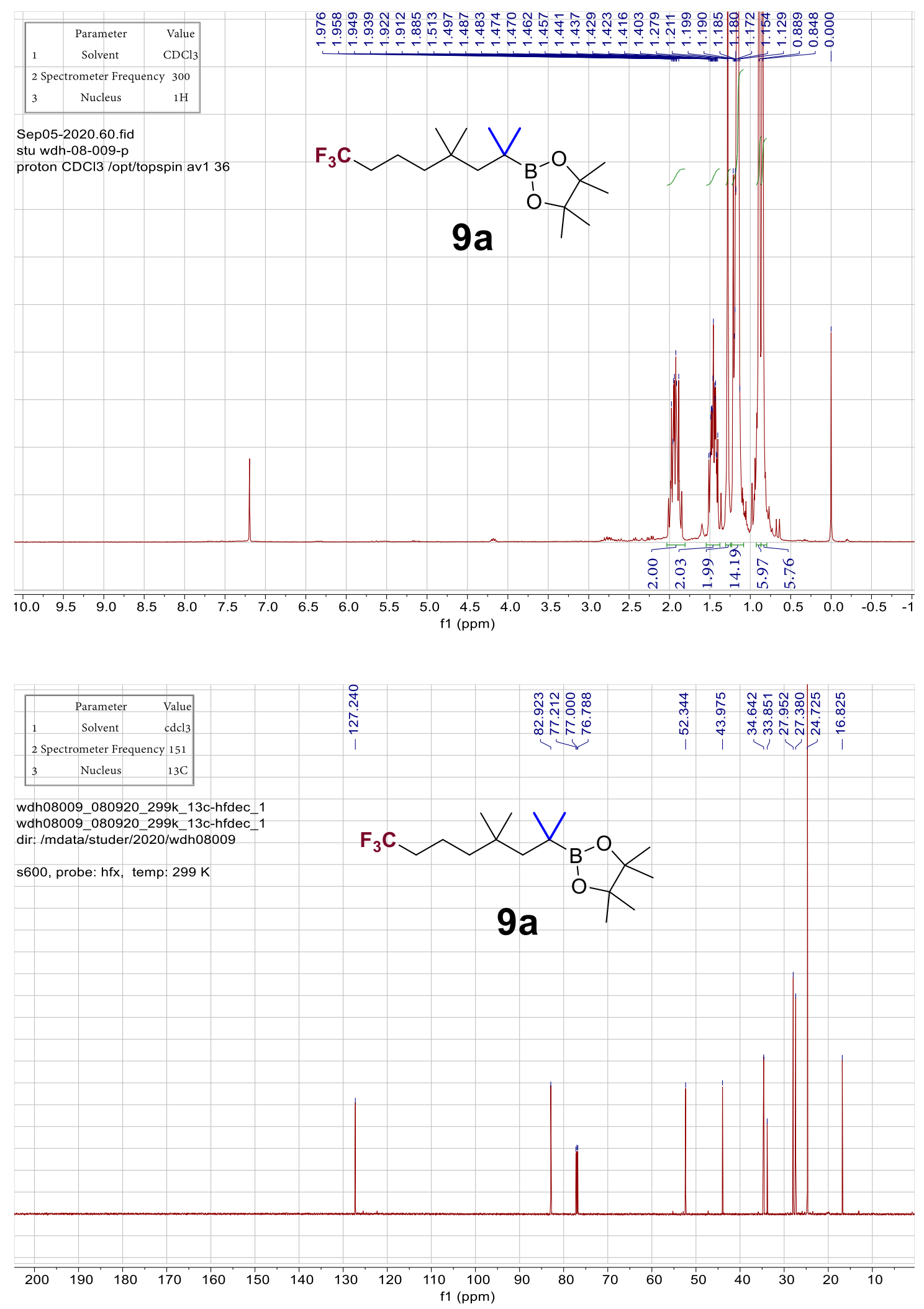

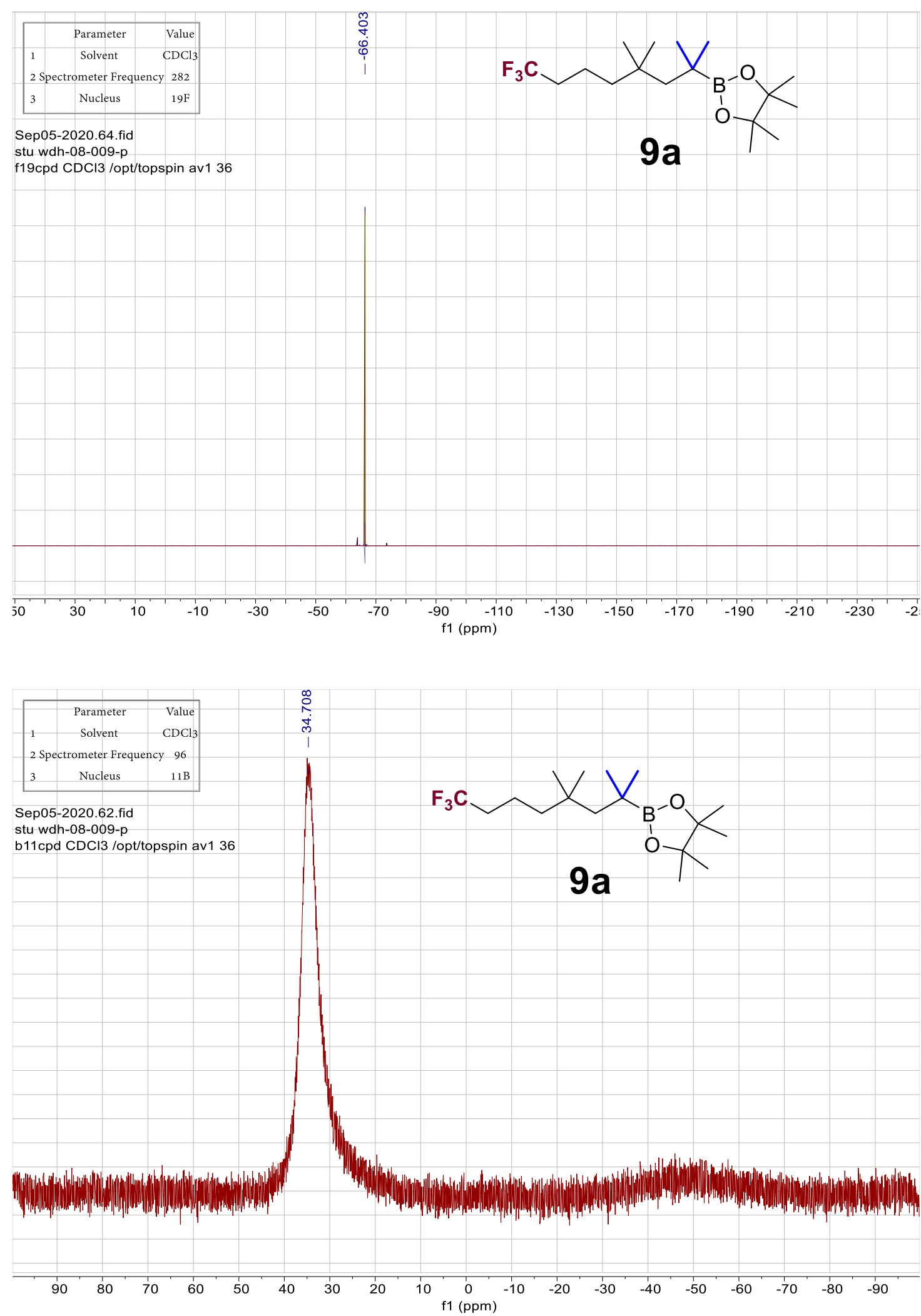

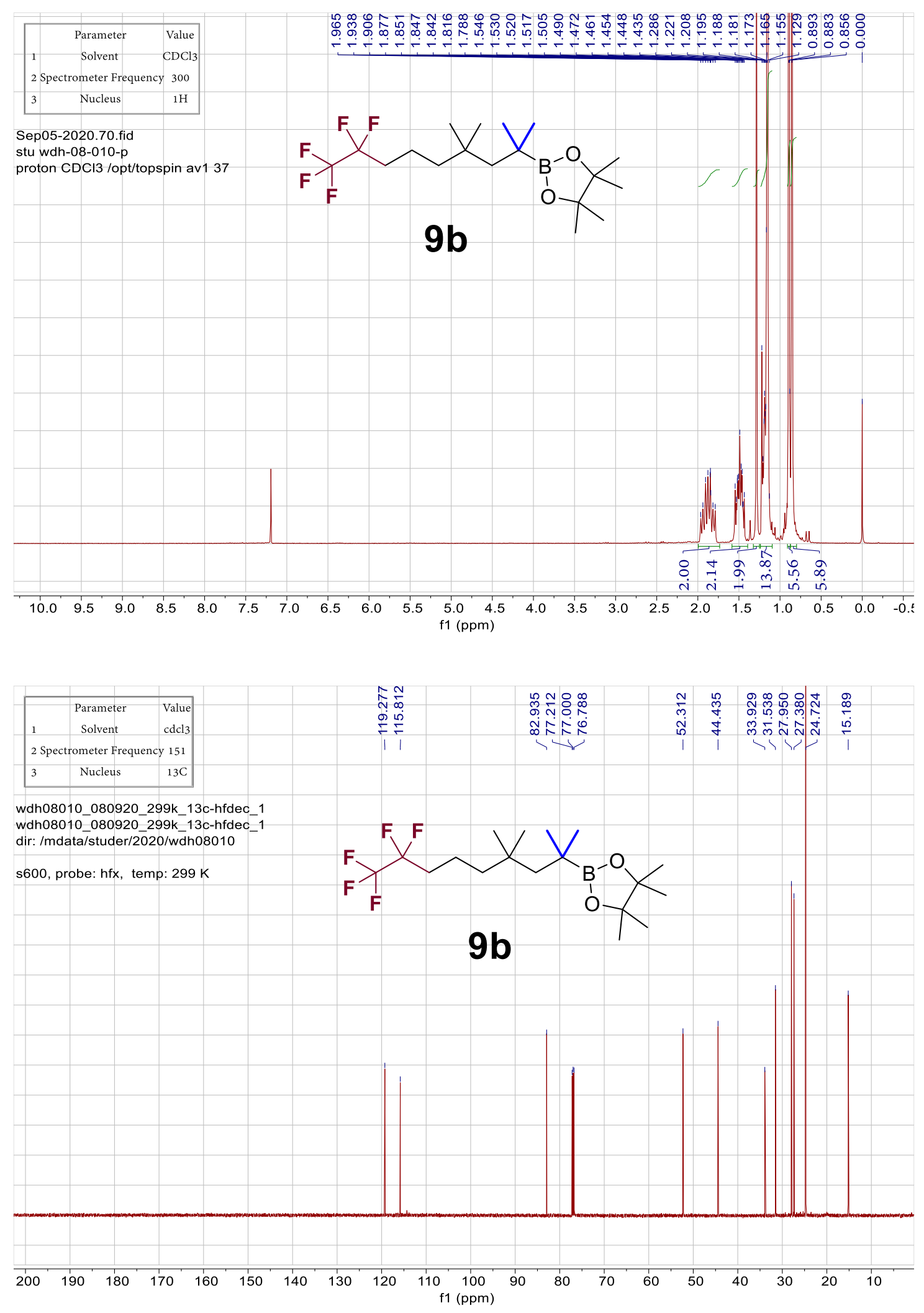

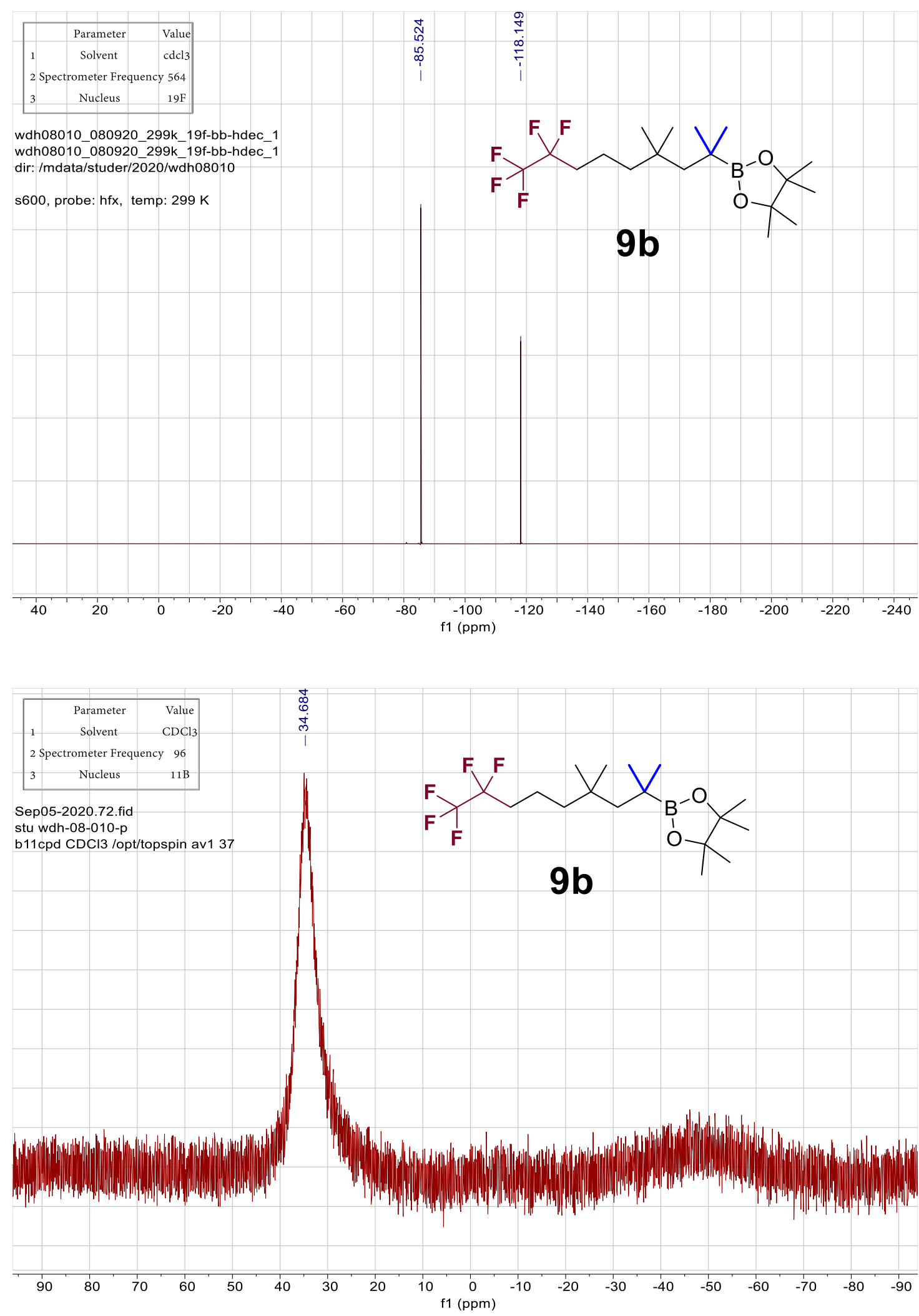

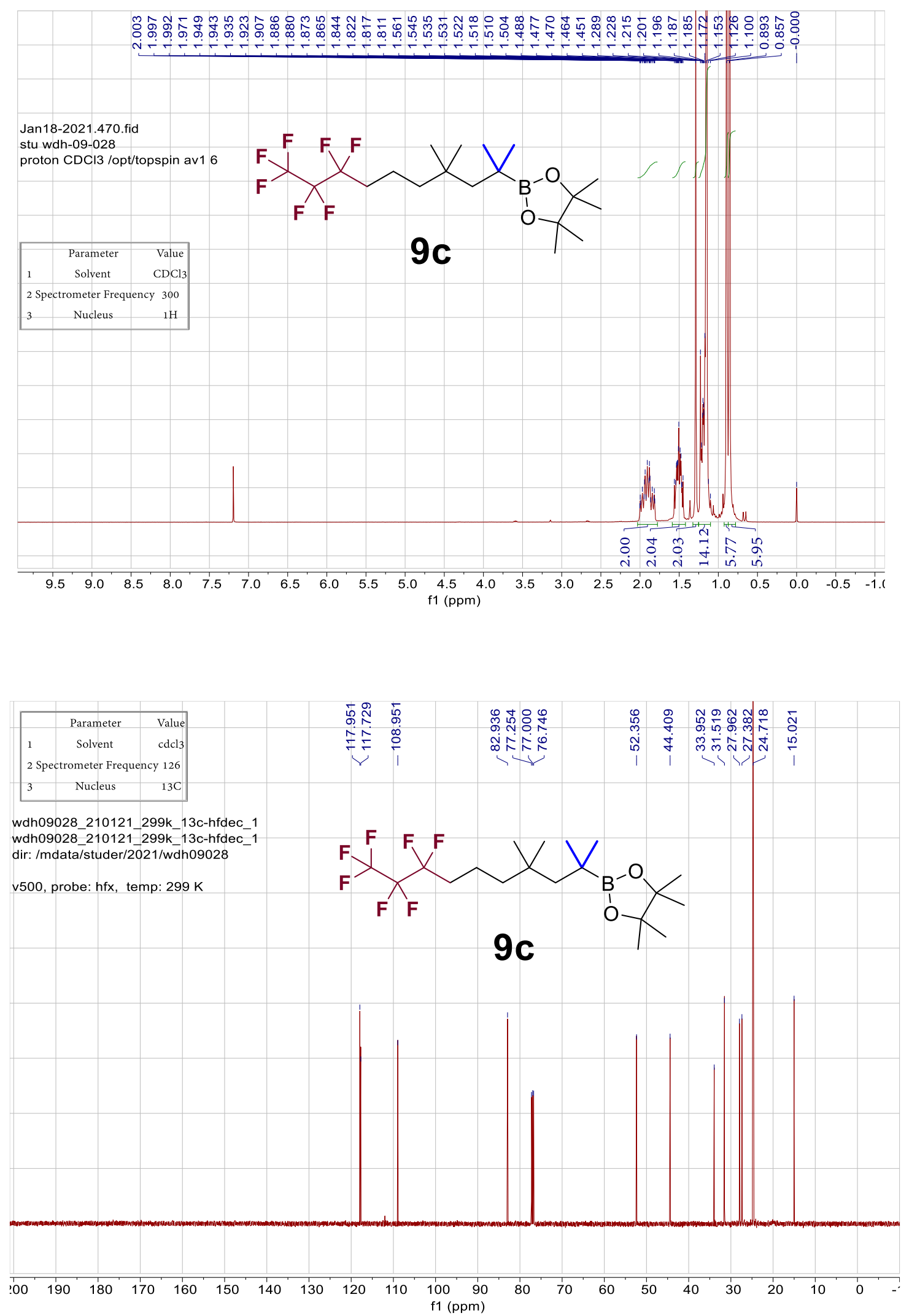

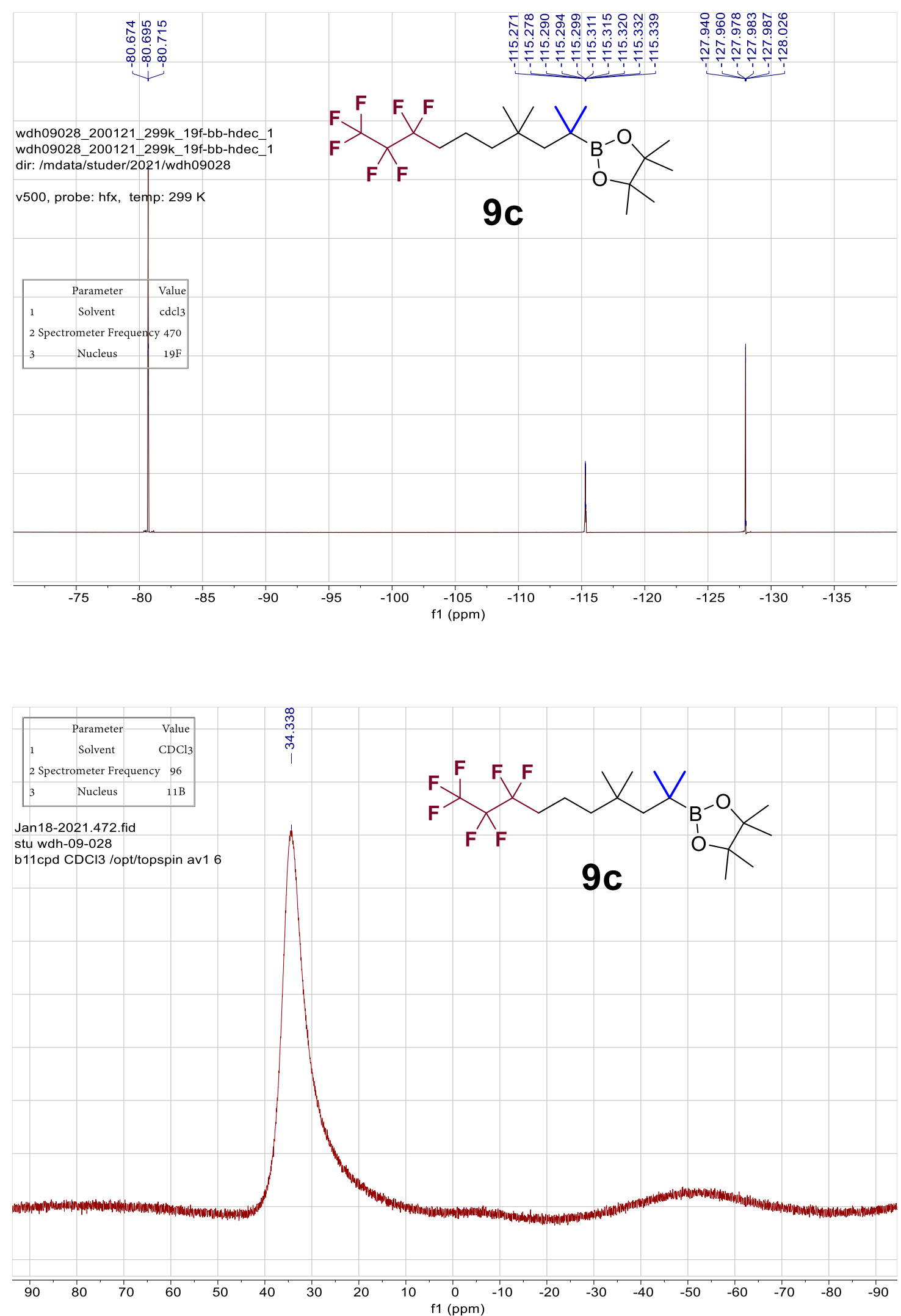

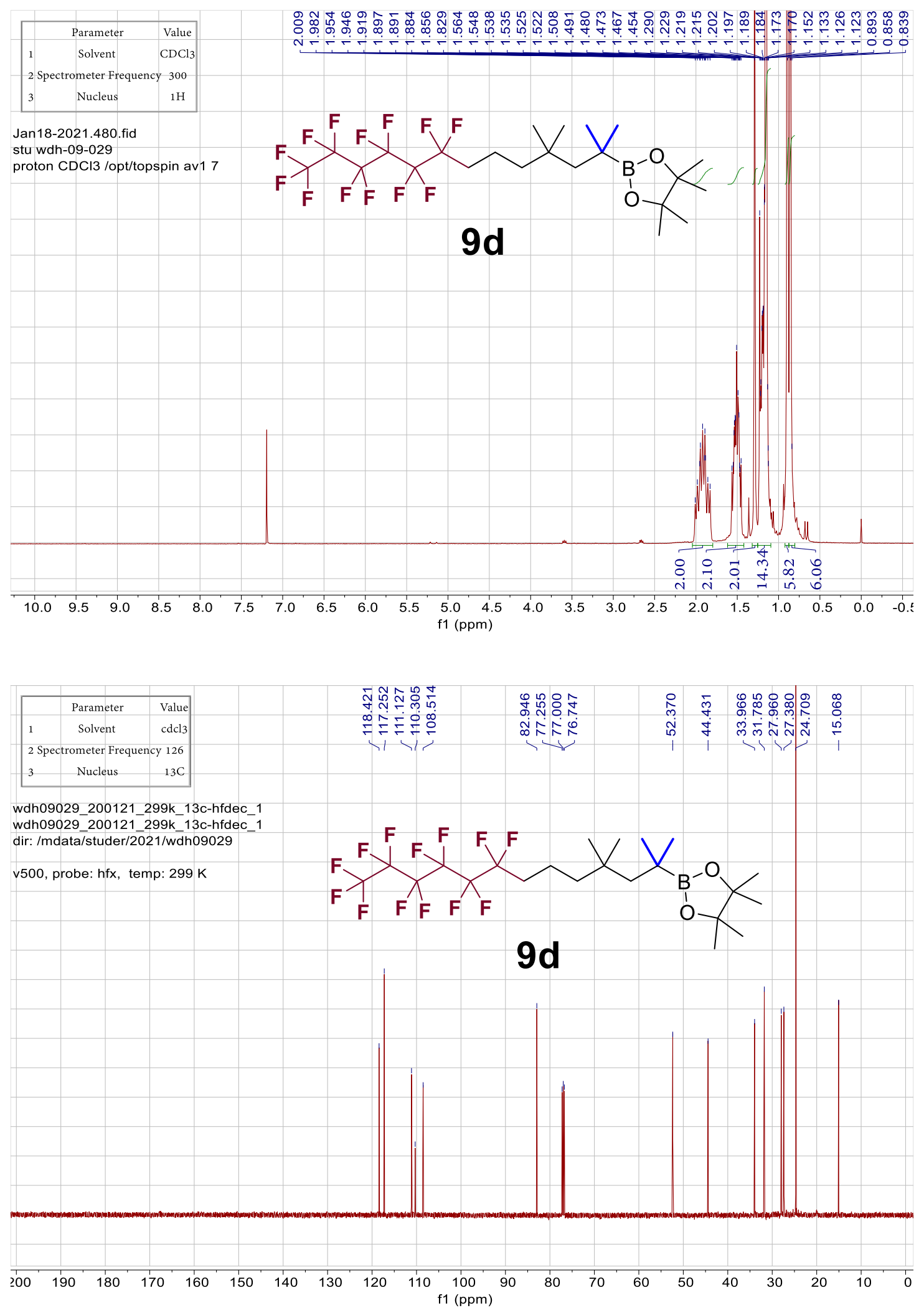

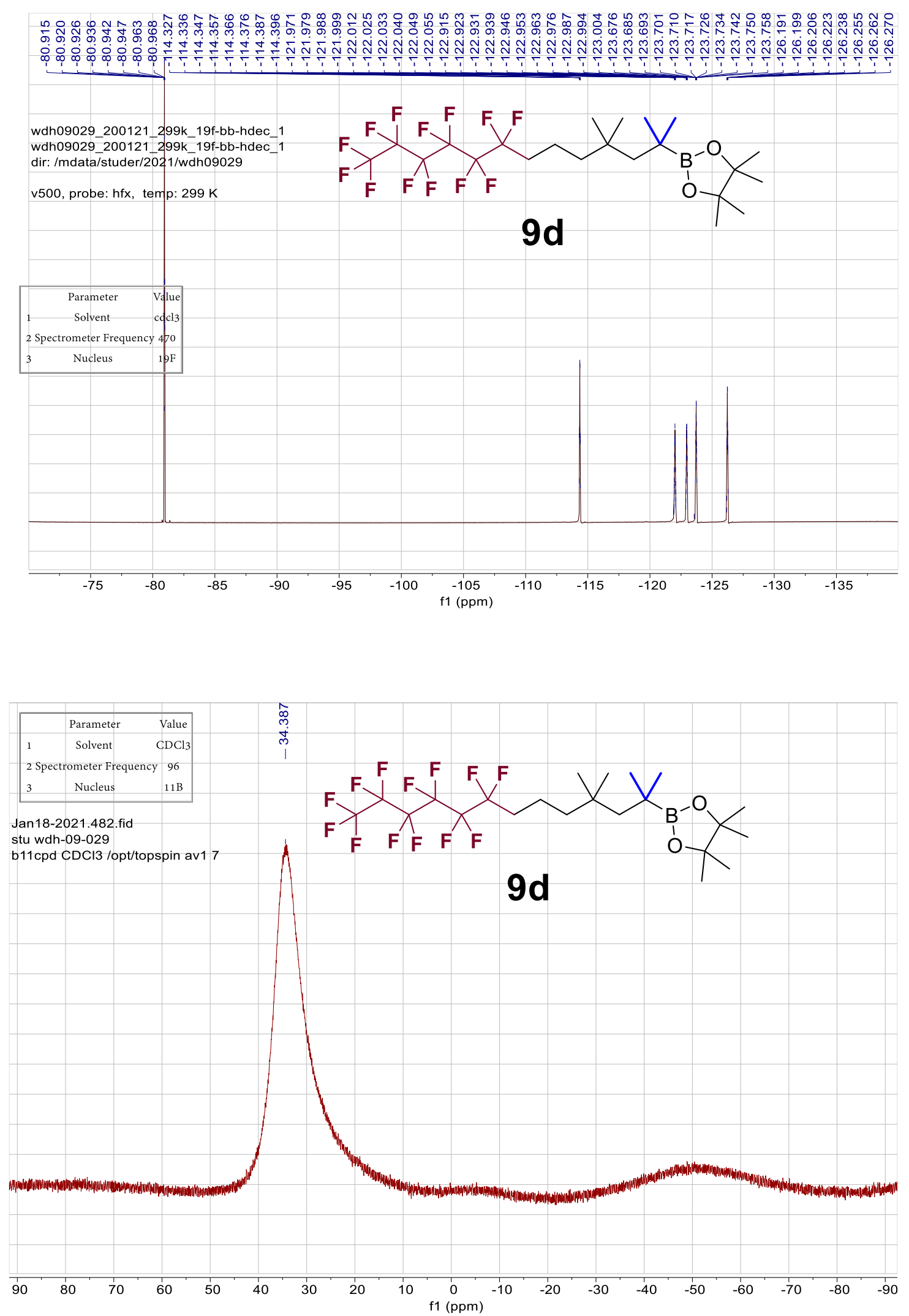

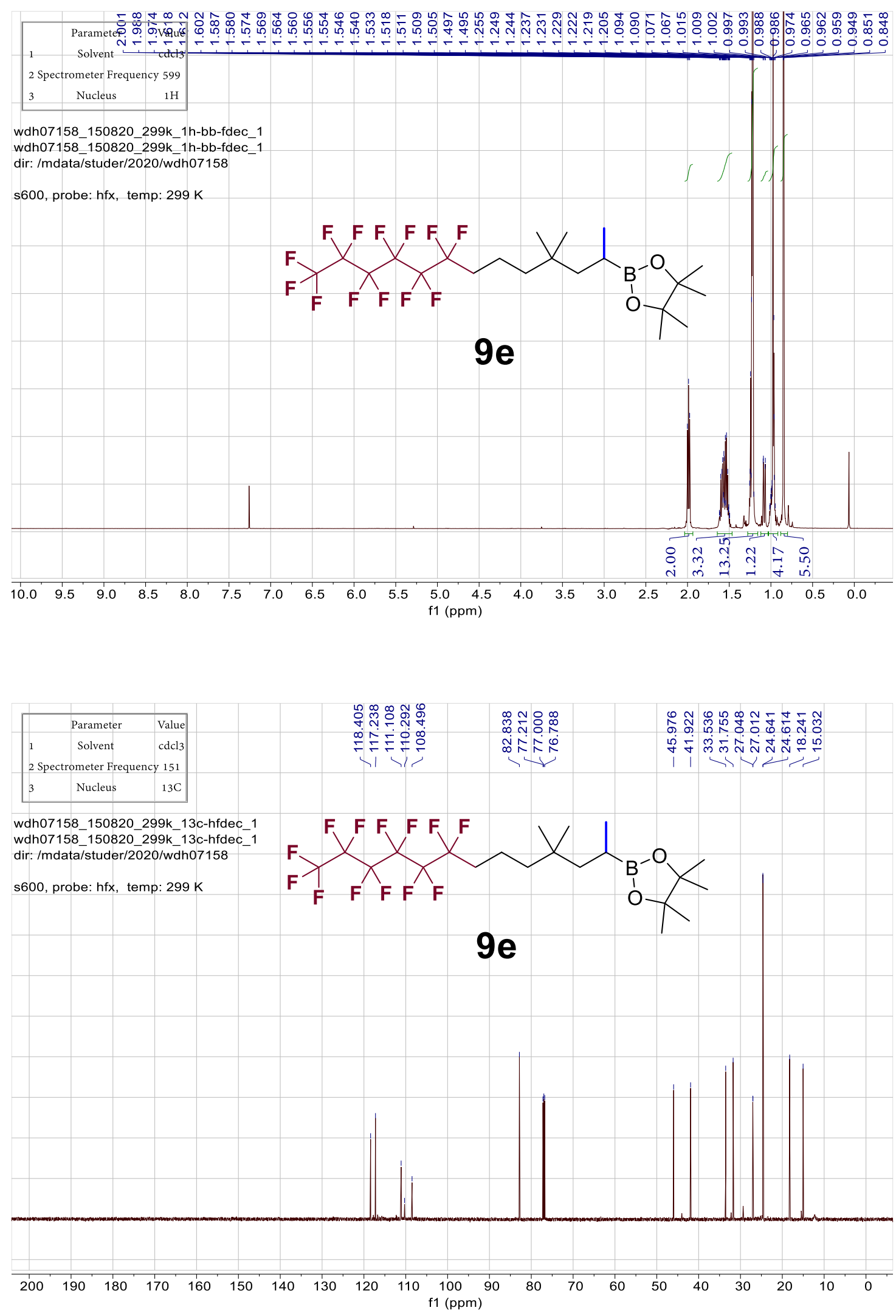


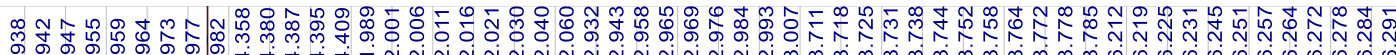

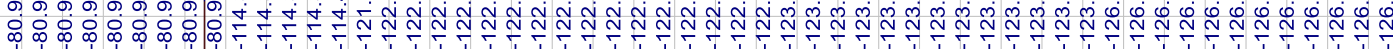

dh07158 150820 299k 19f-bb-hdec

wdh07158 150820 299k $19 \mathrm{f}-\mathrm{bb}$-hdec

dir:/mdata/studer/2020/wdh07158

s600, probe: hfx, tenpp: $299 \mathrm{~K}$<smiles>CC(C)CC(C)(C)CCCC(F)(F)C(F)(F)C(F)(F)C(F)(F)C(F)(F)C(F)(F)F</smiles>

$9 e$
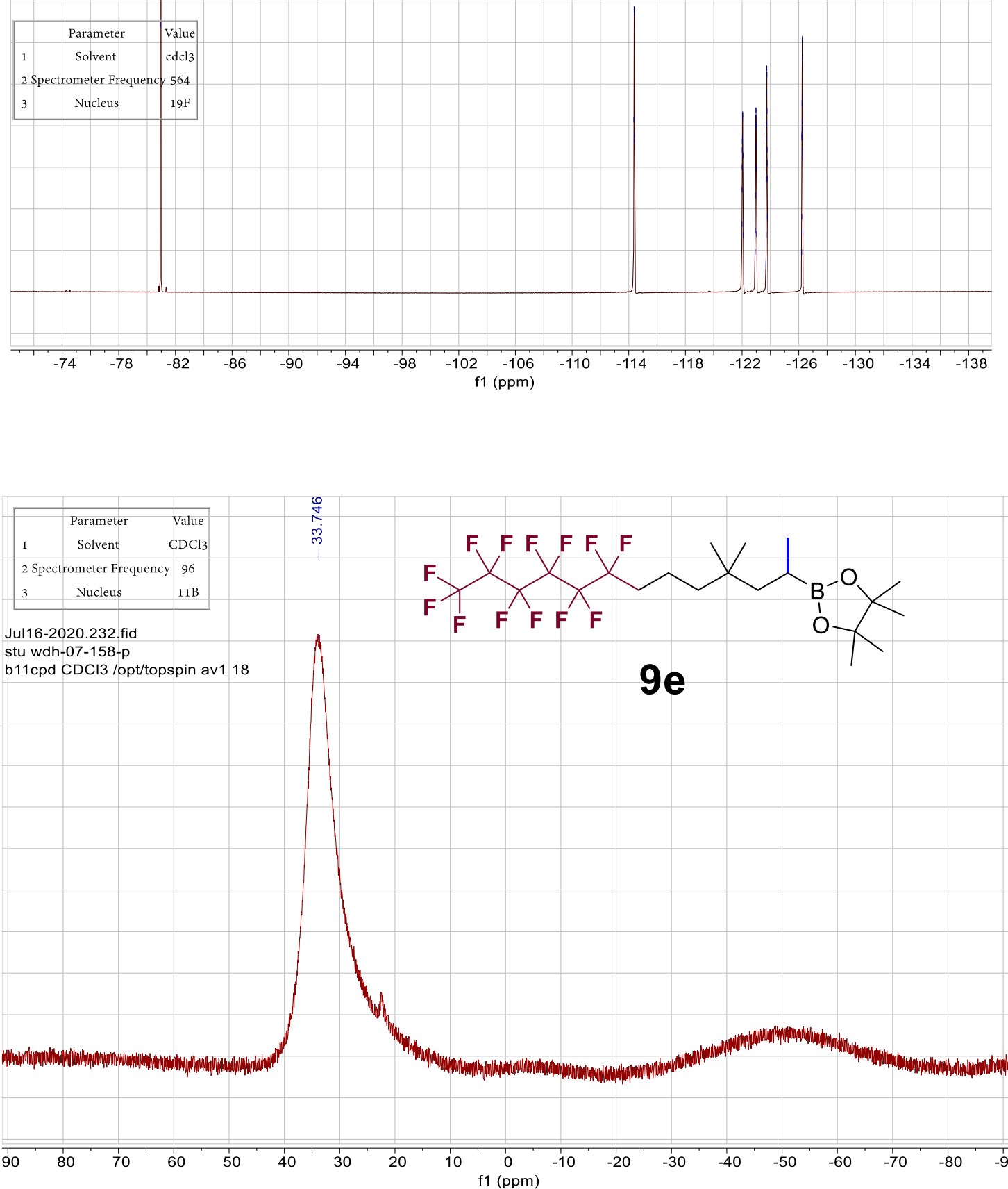

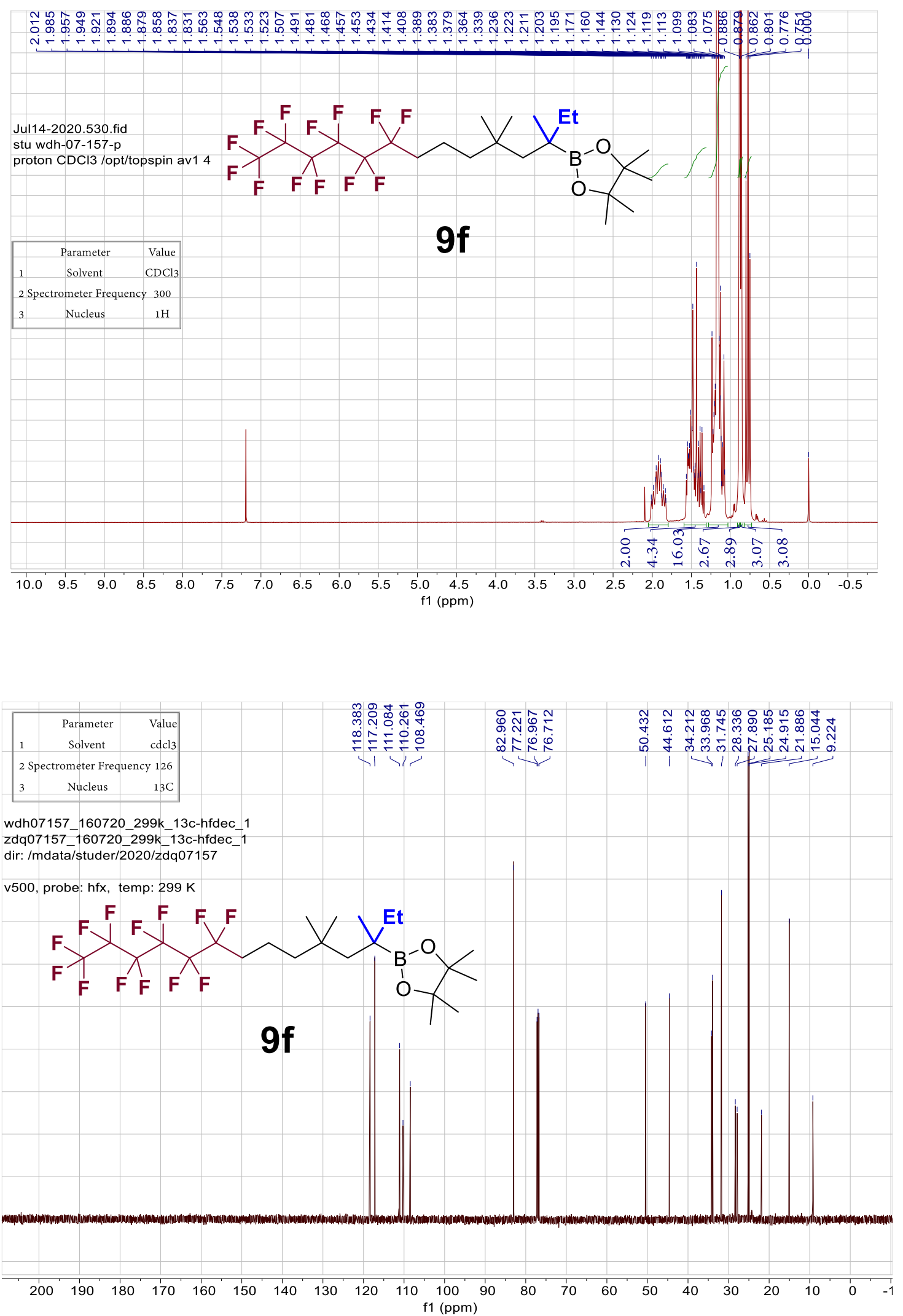

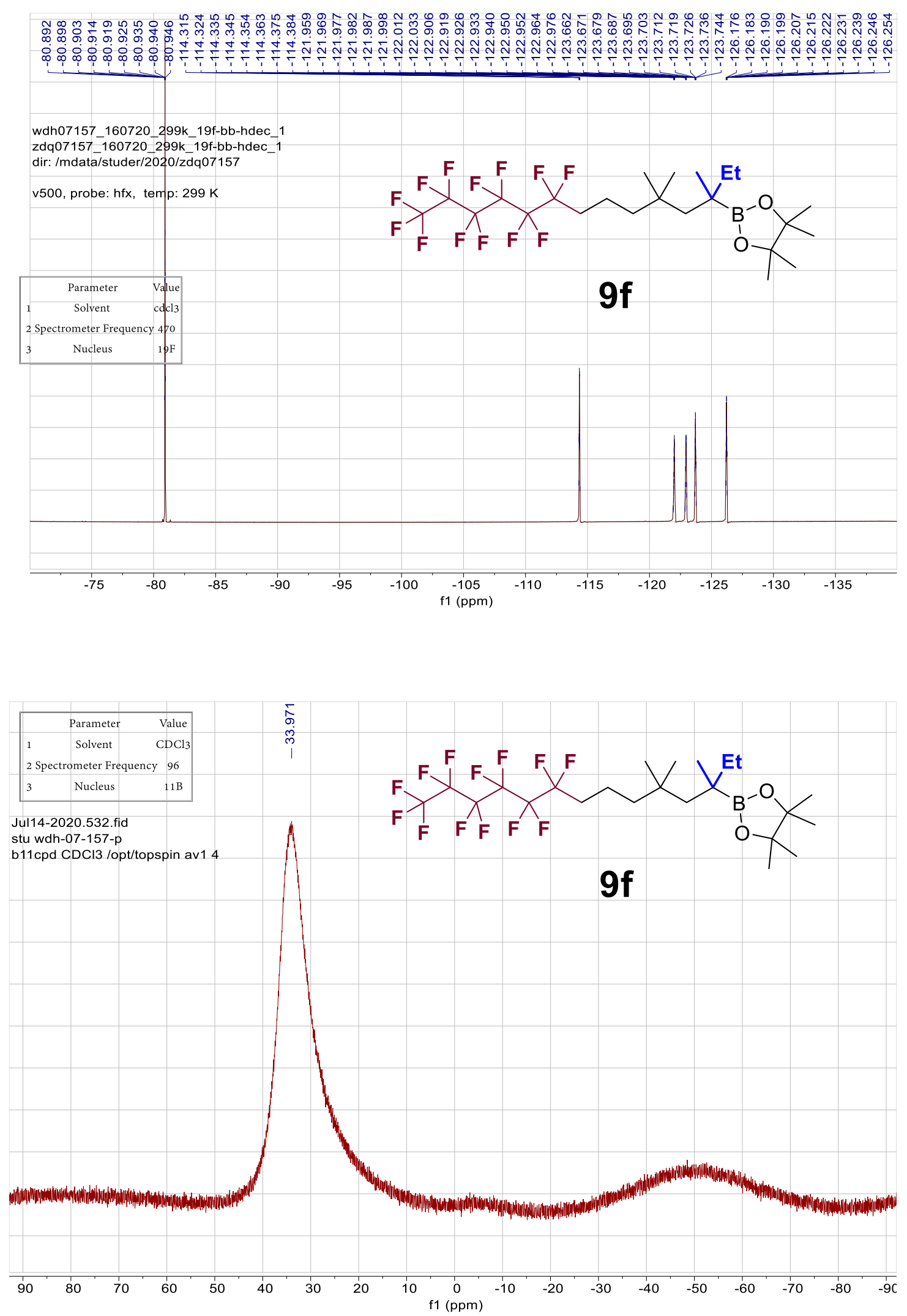

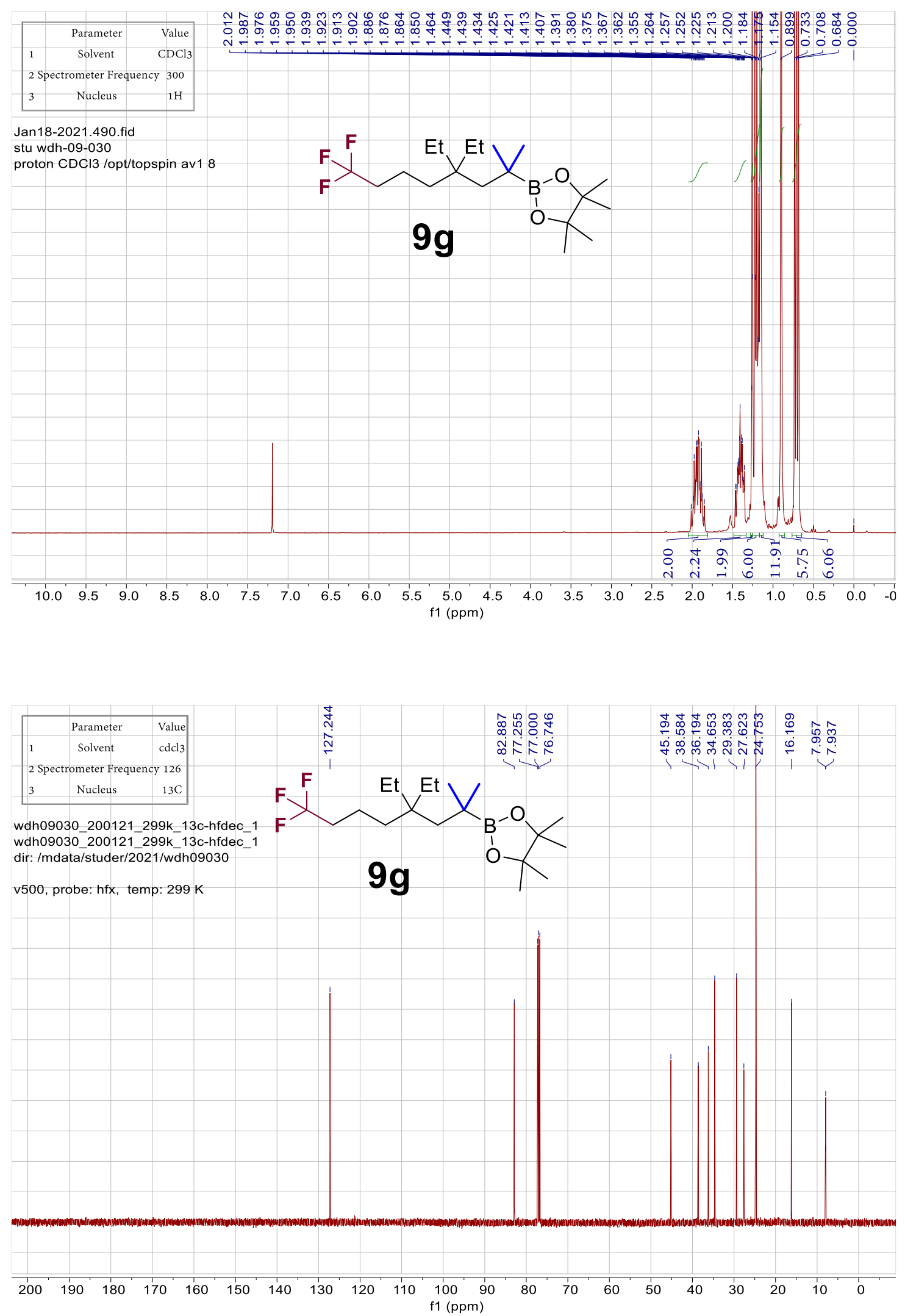

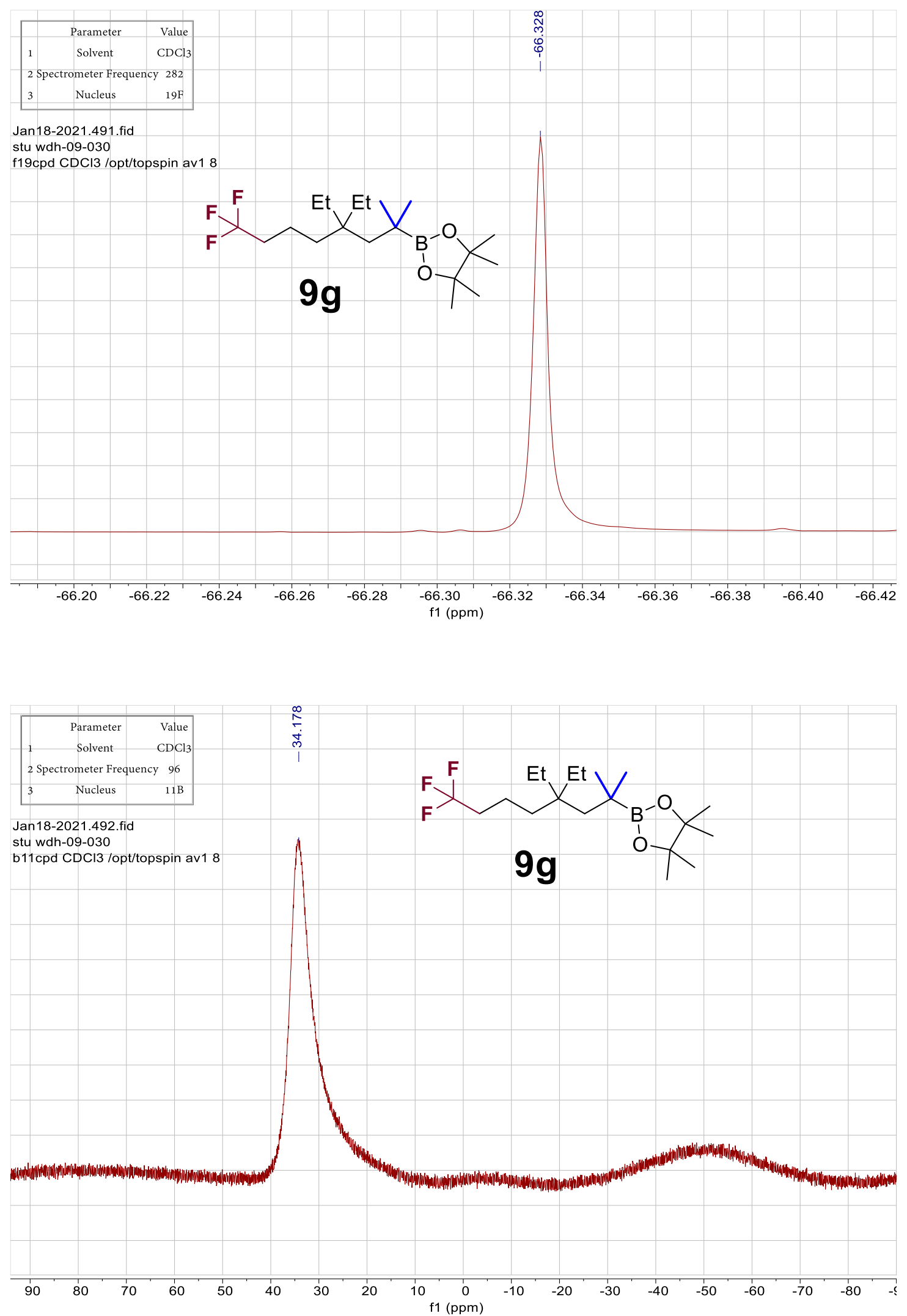

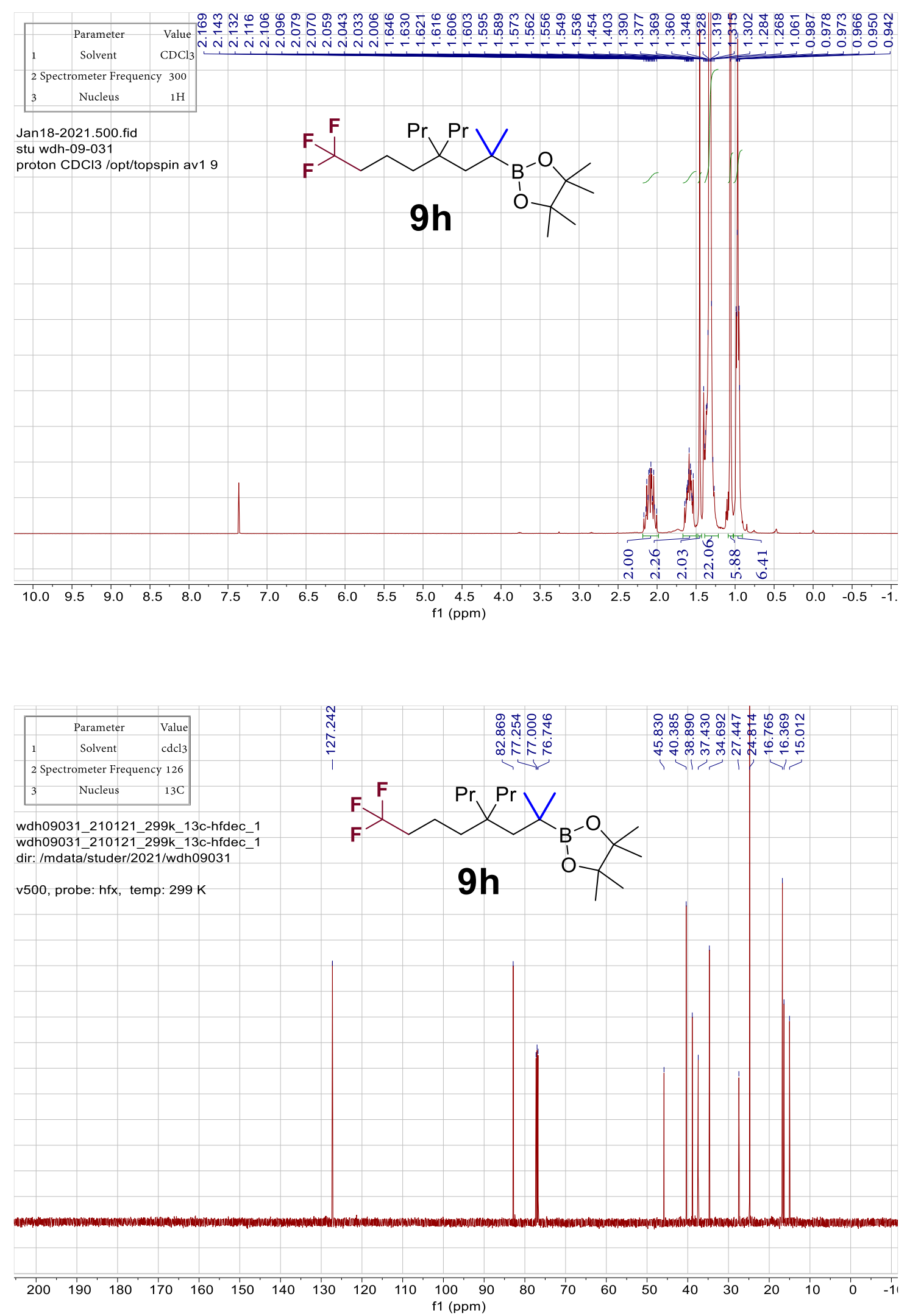

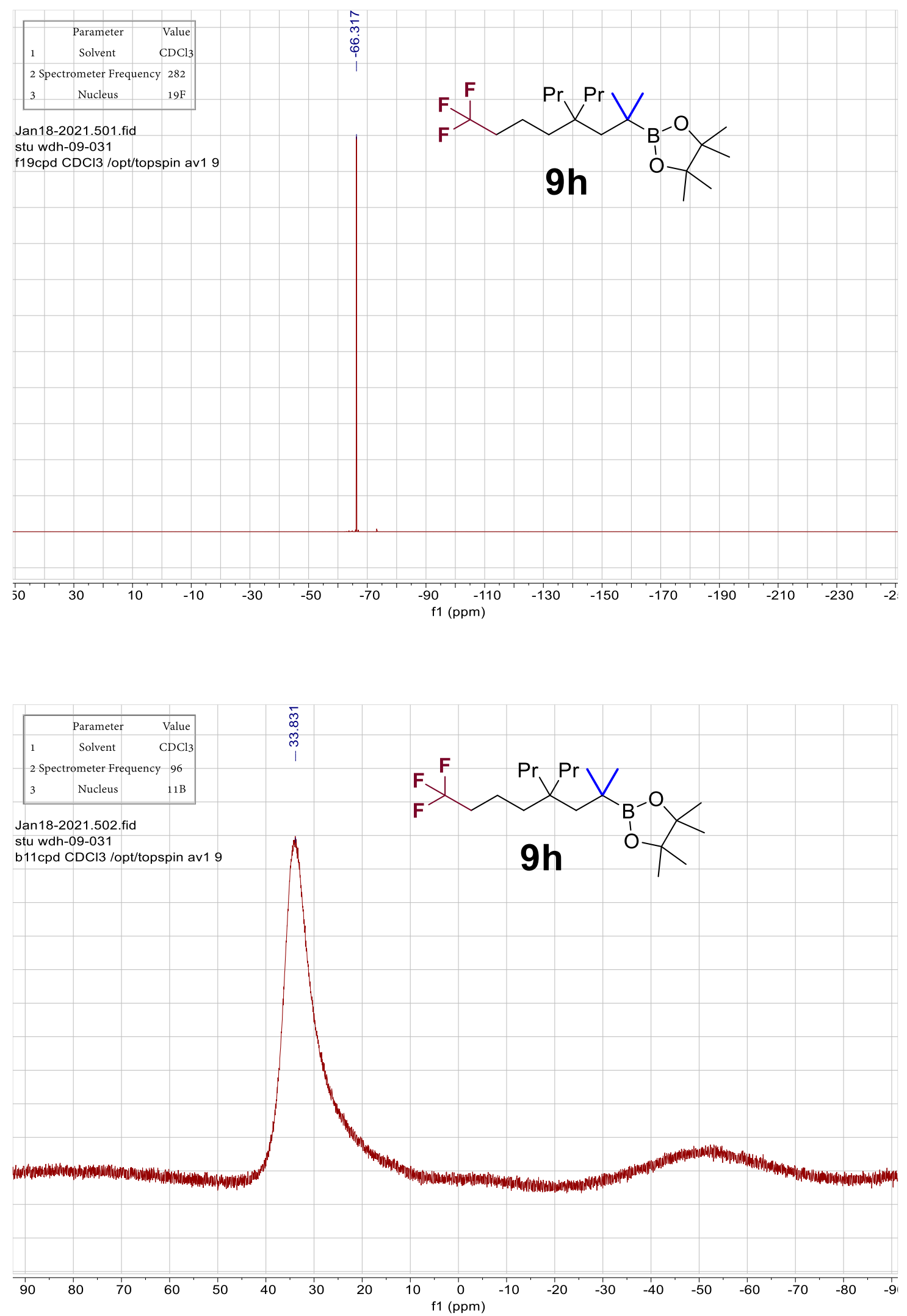

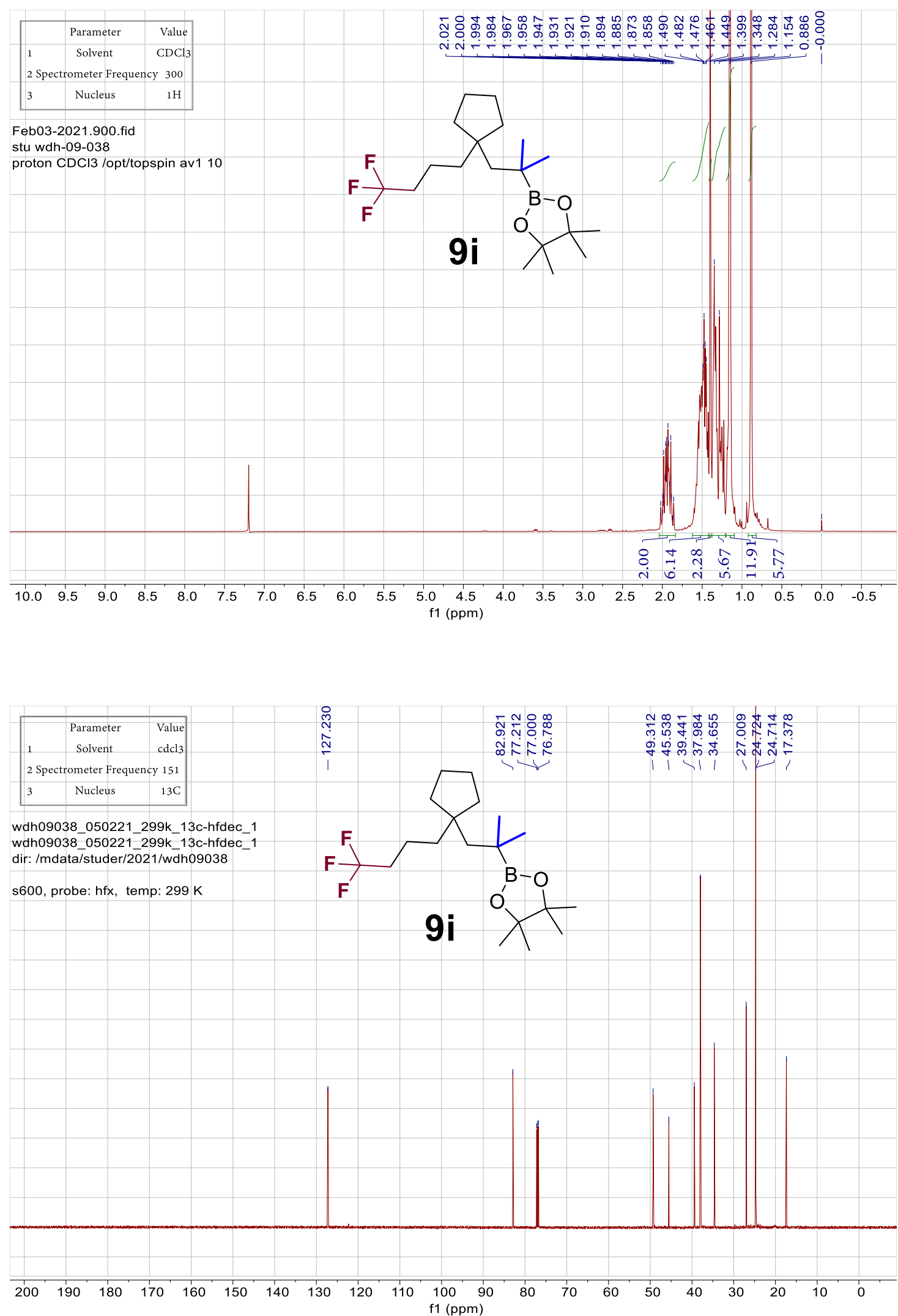

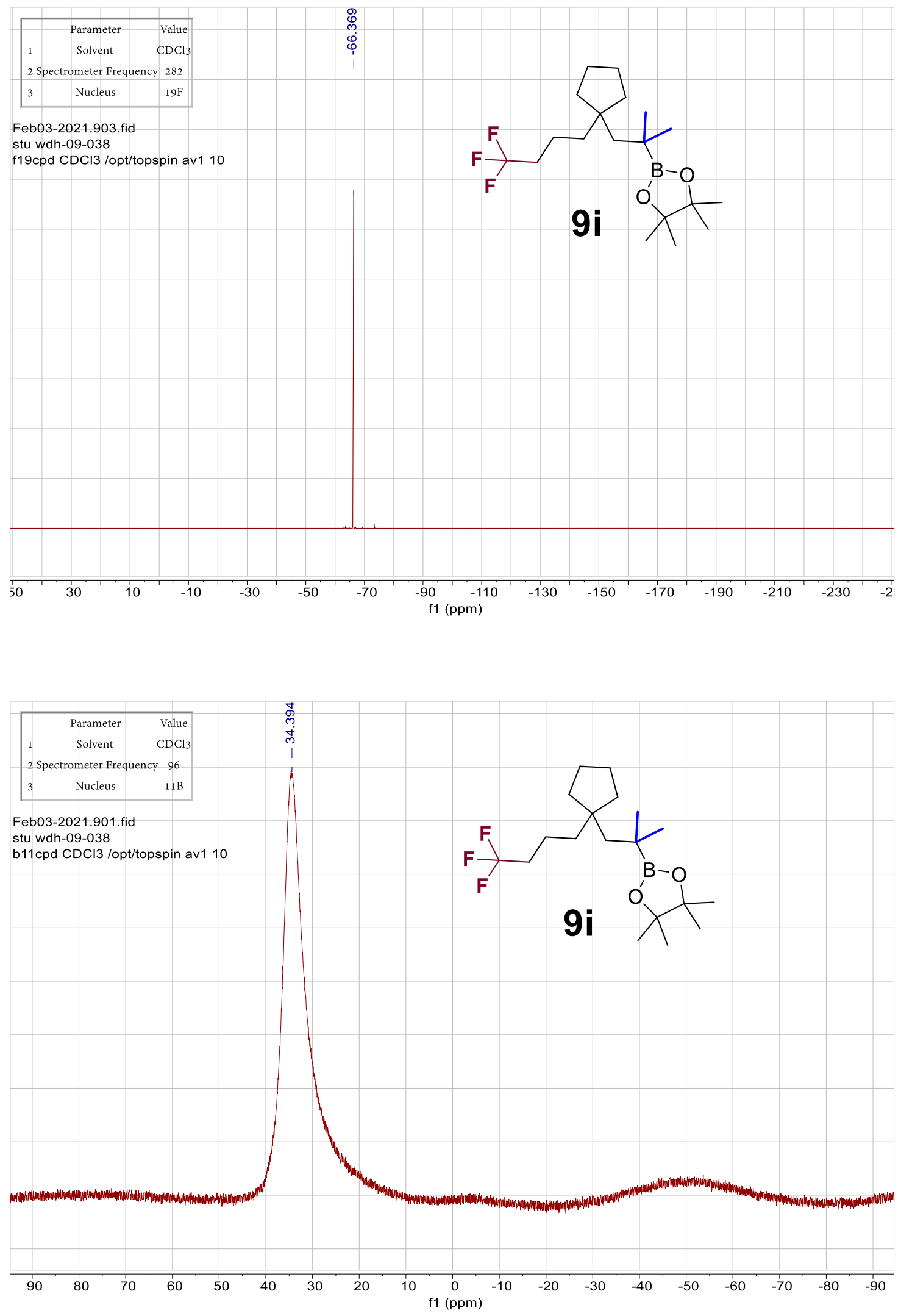

S109 

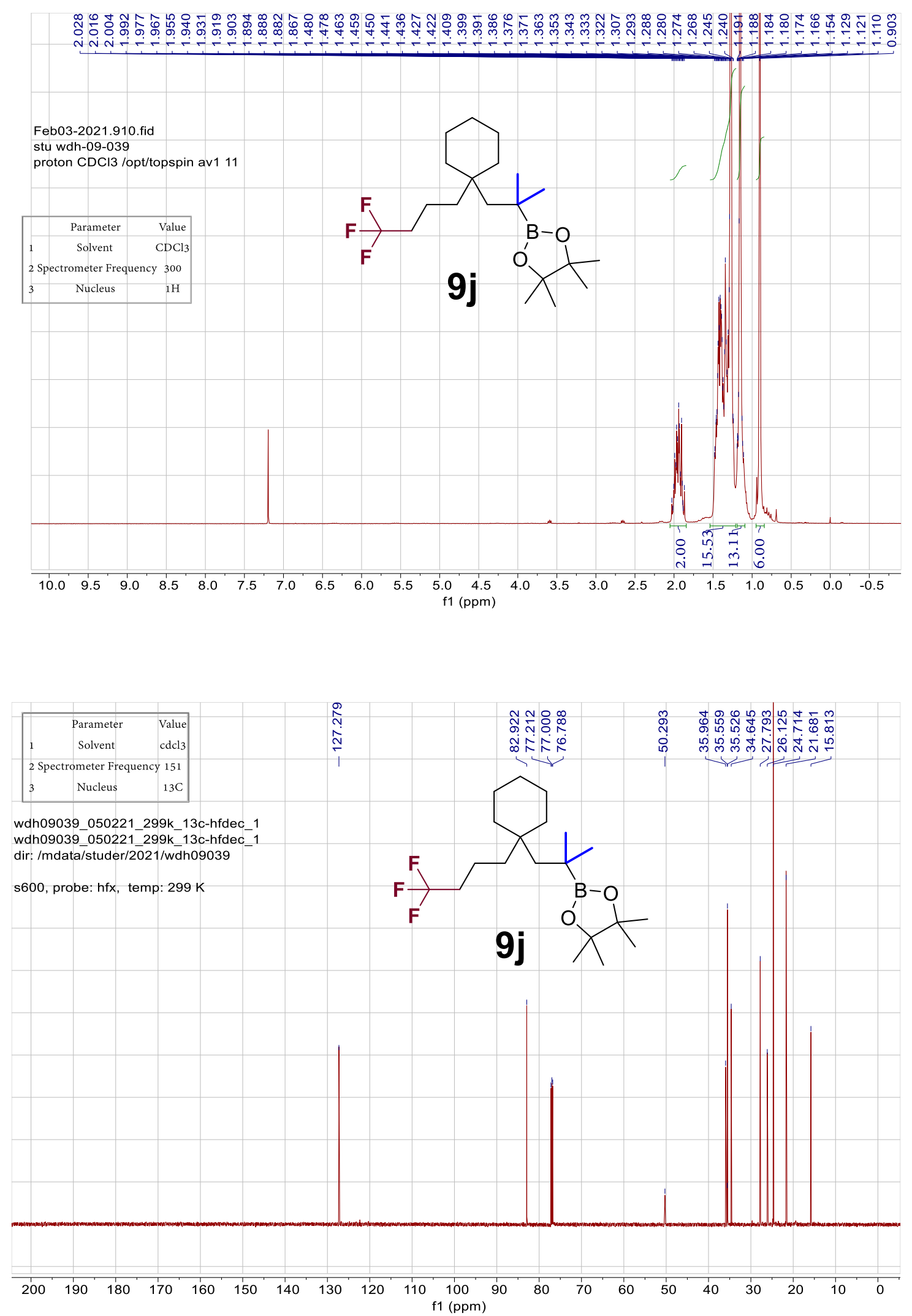

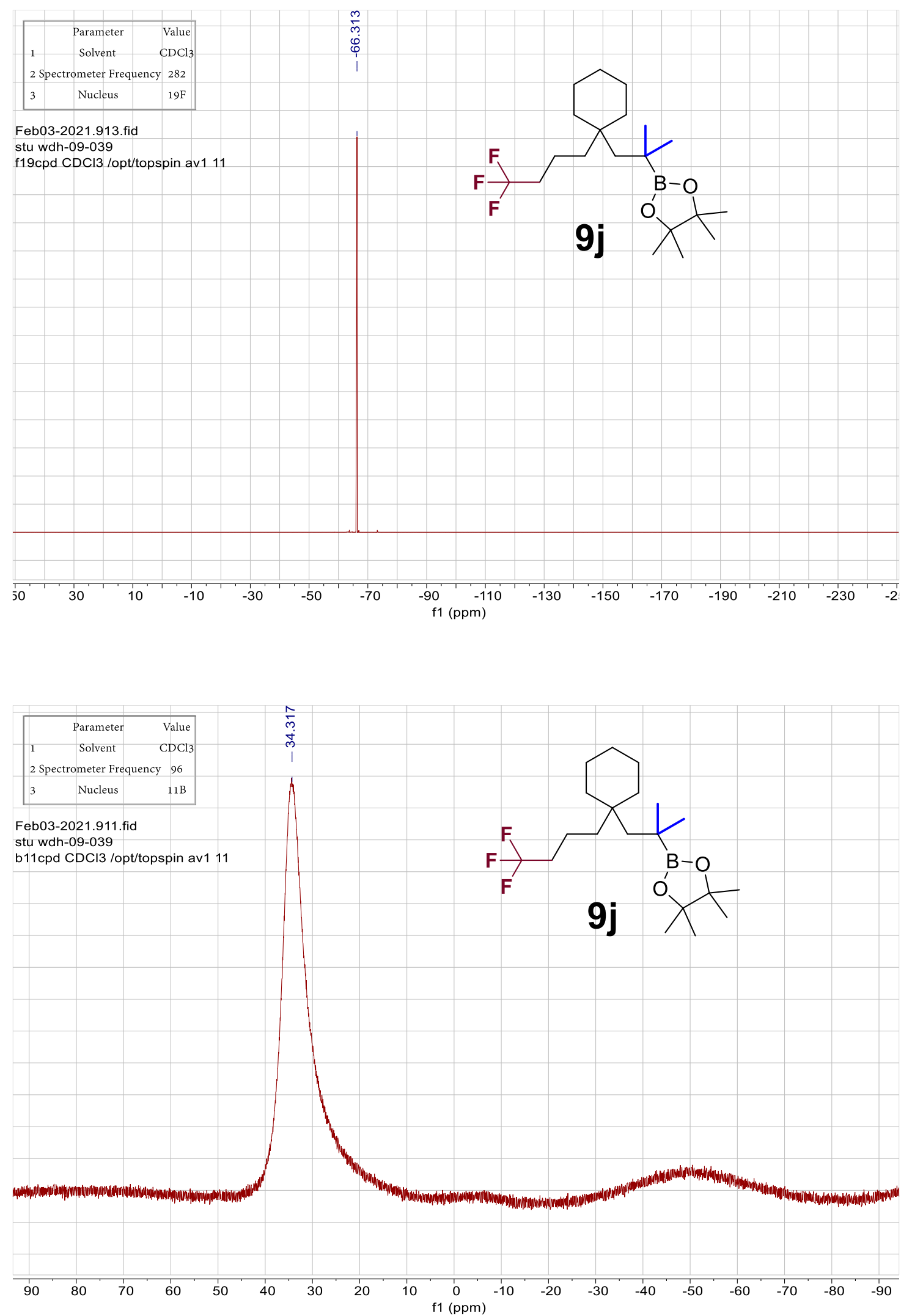

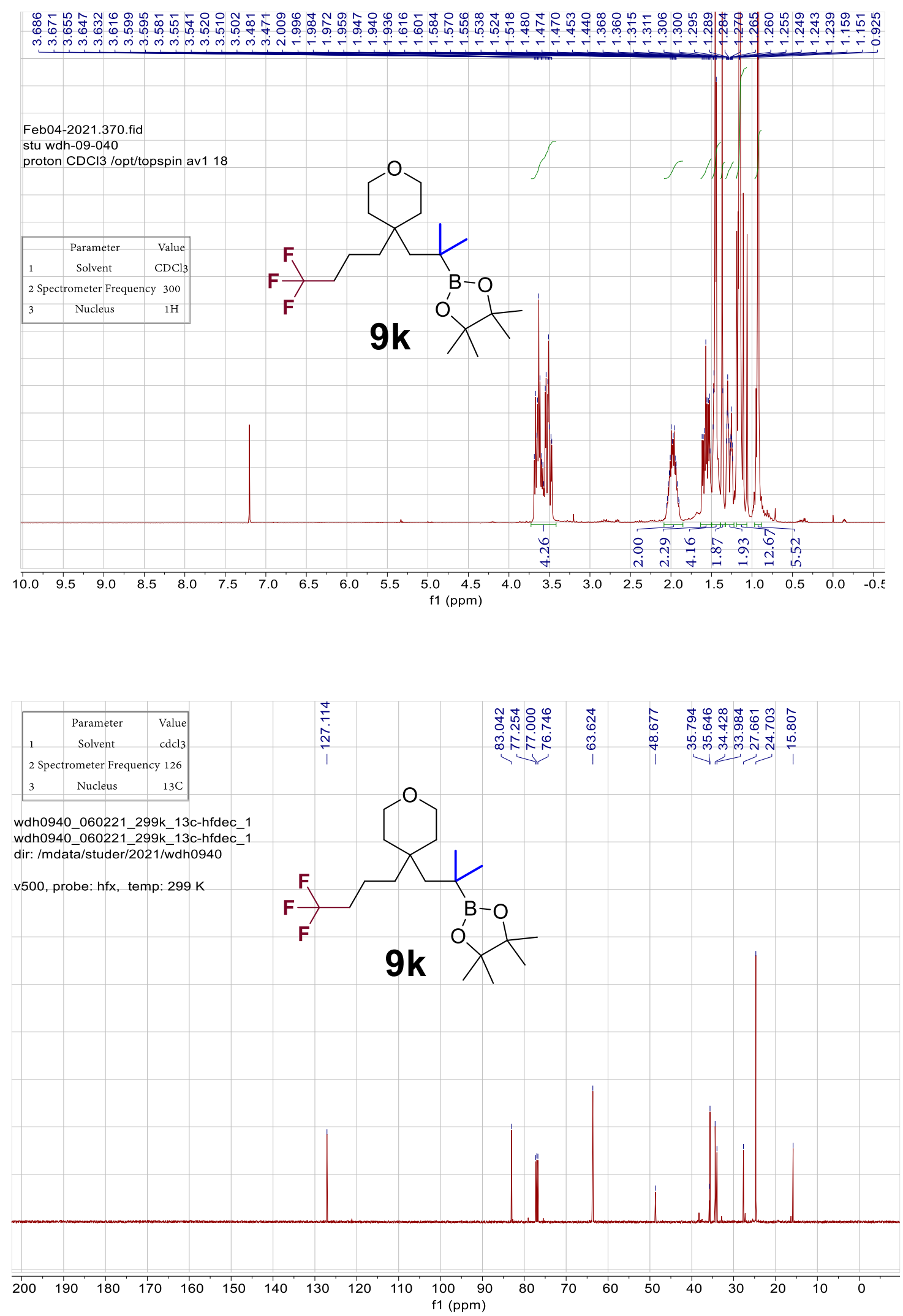

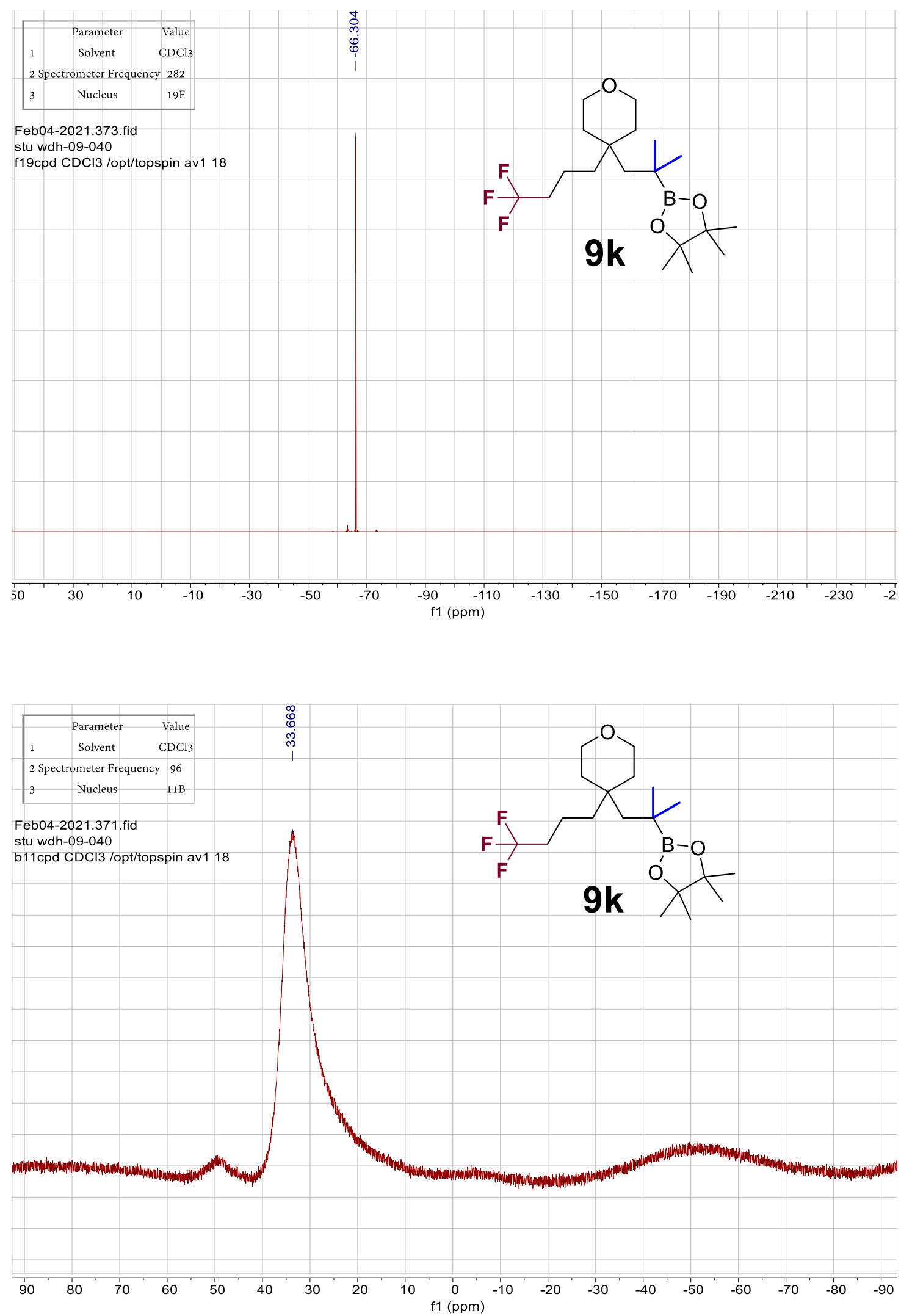

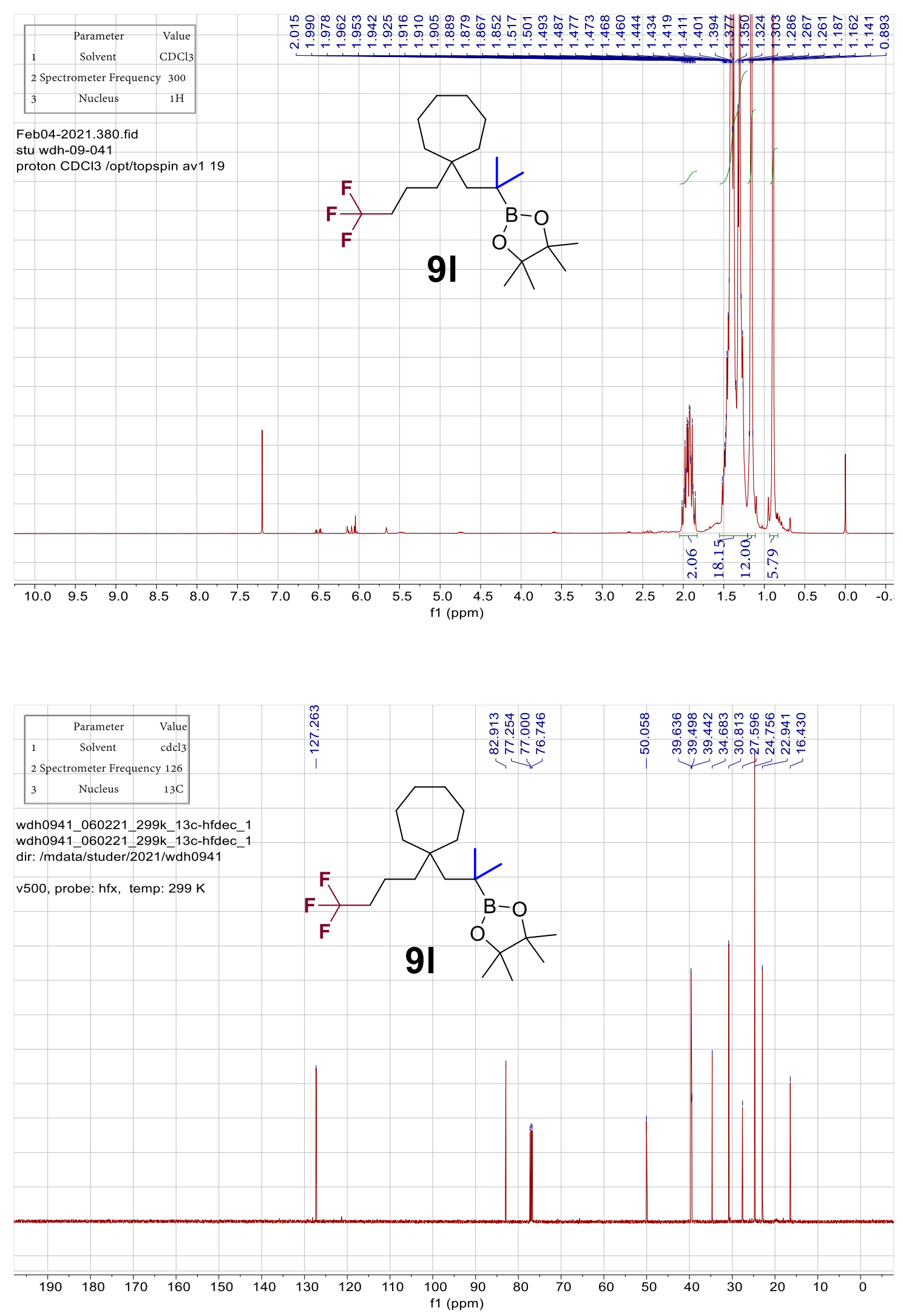

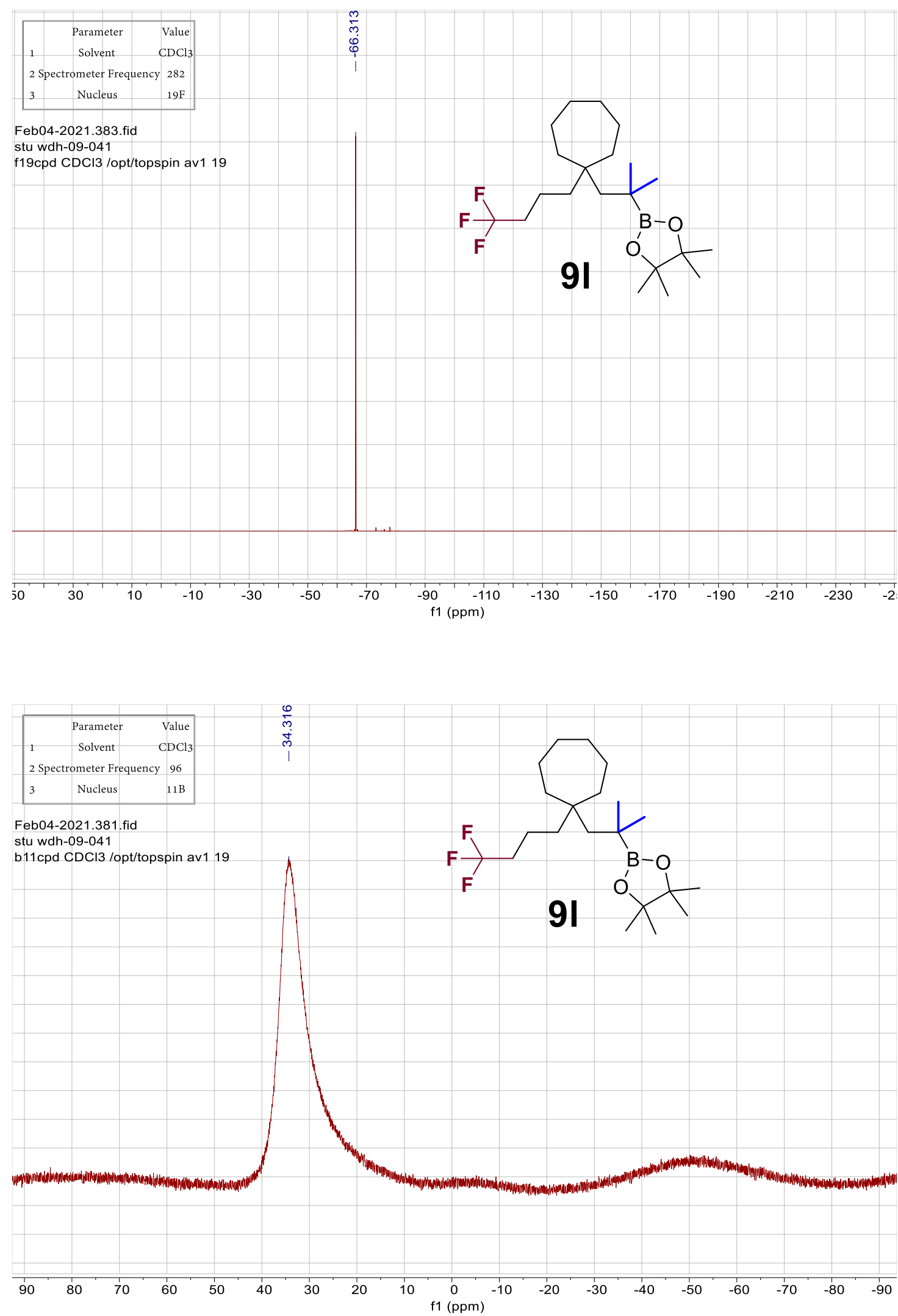

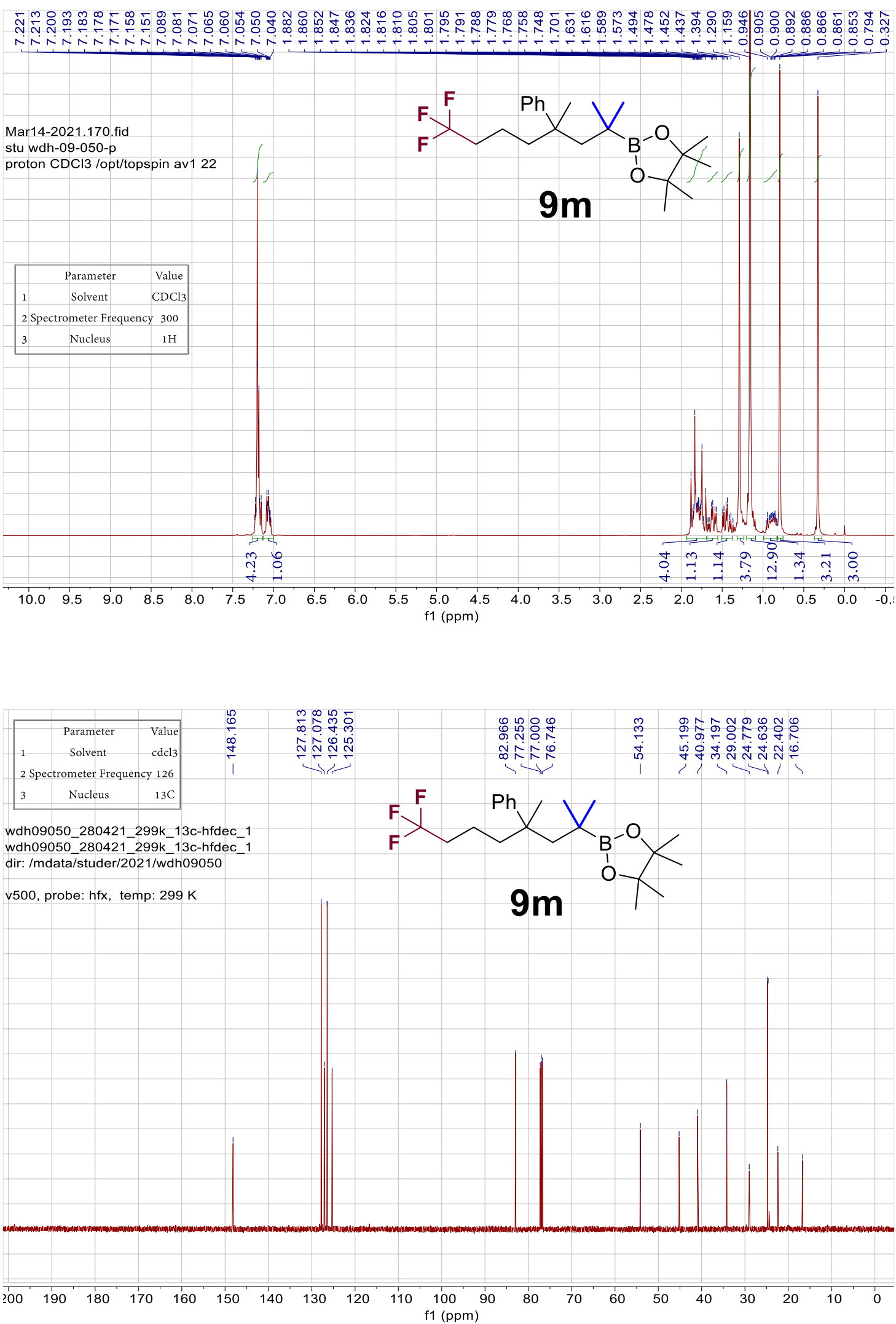

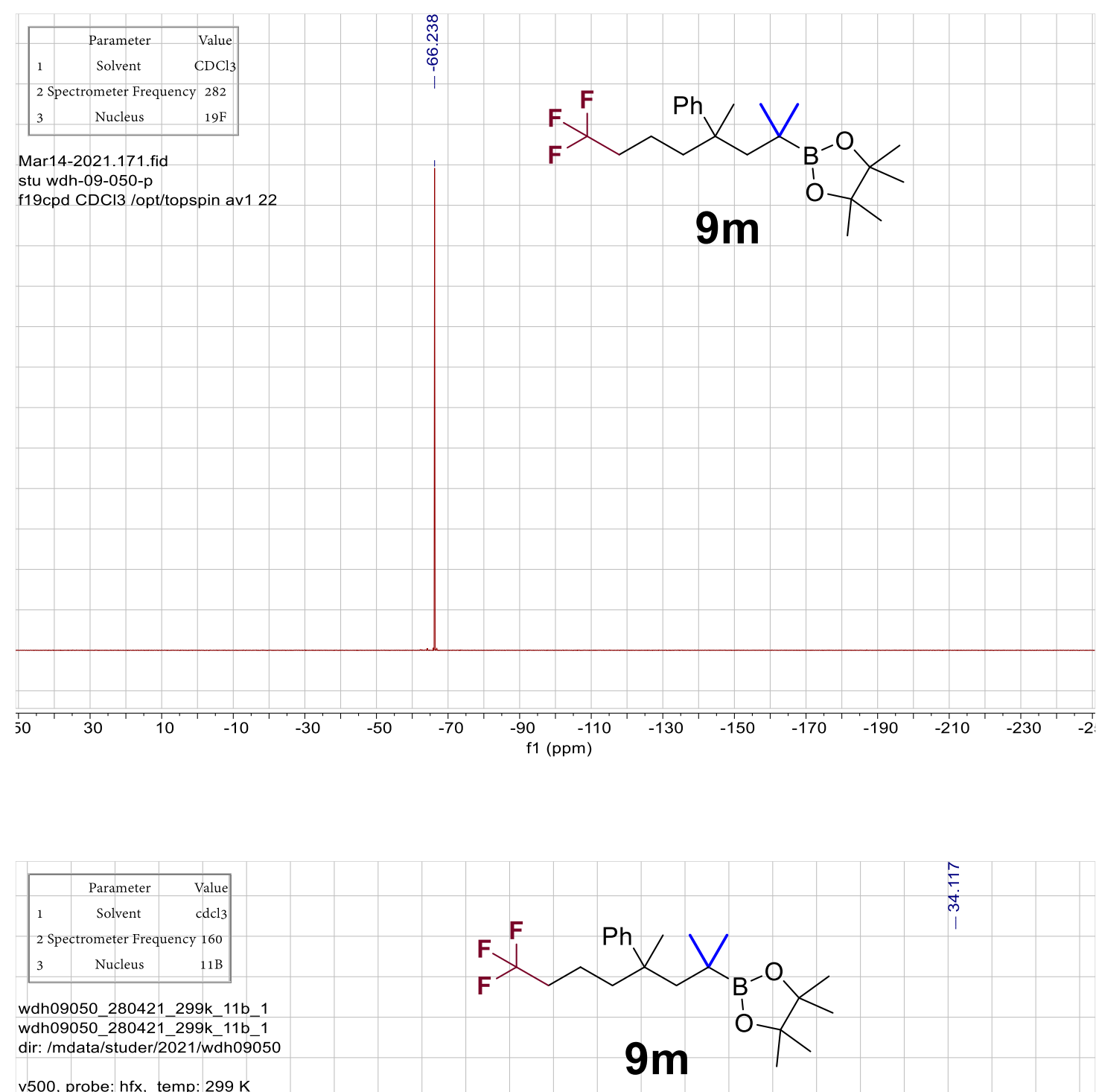

v500, probe: $\mathrm{hfx}$, temp: $299 \mathrm{~K}$

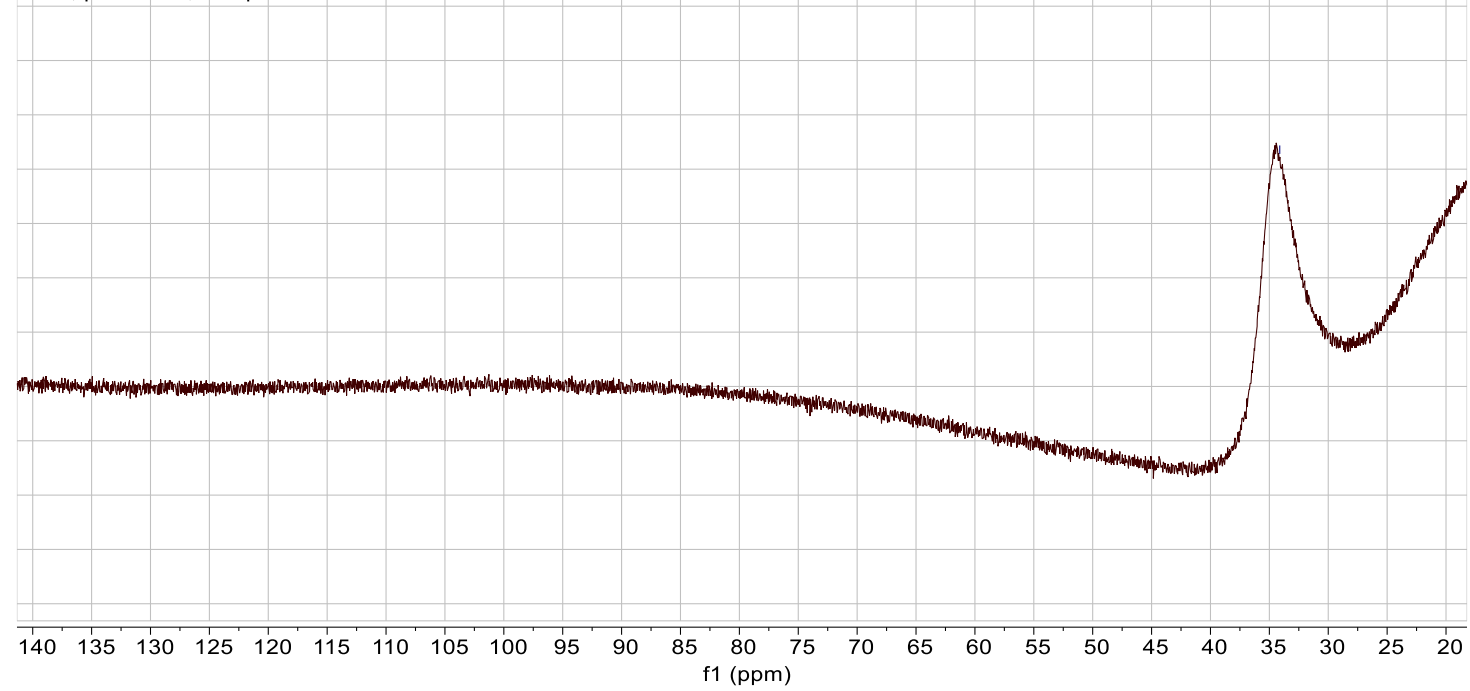



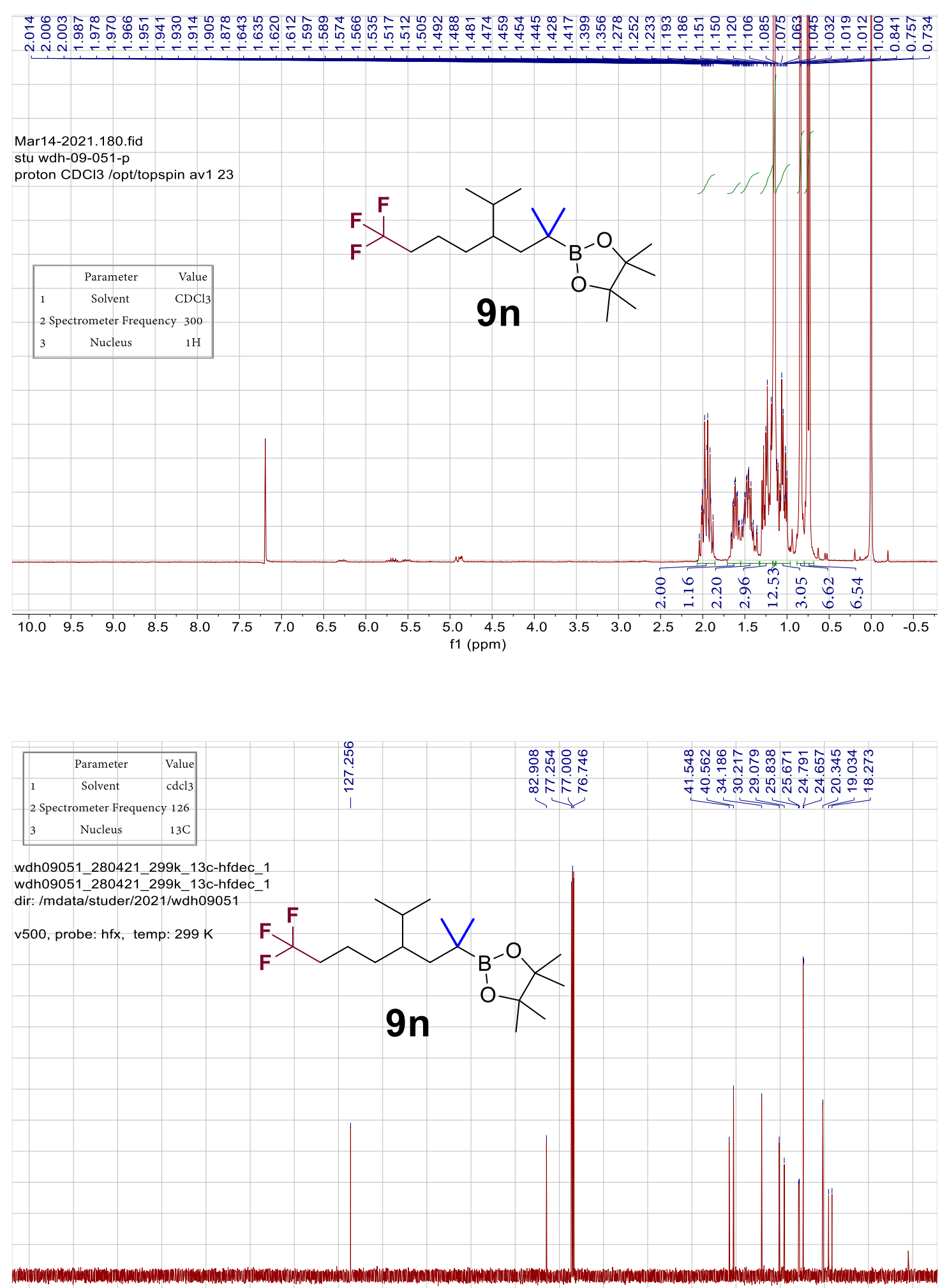

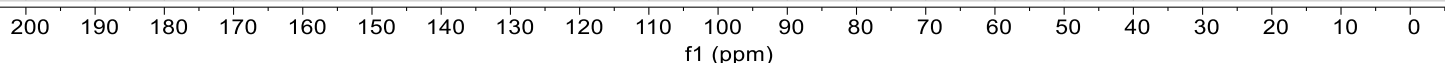



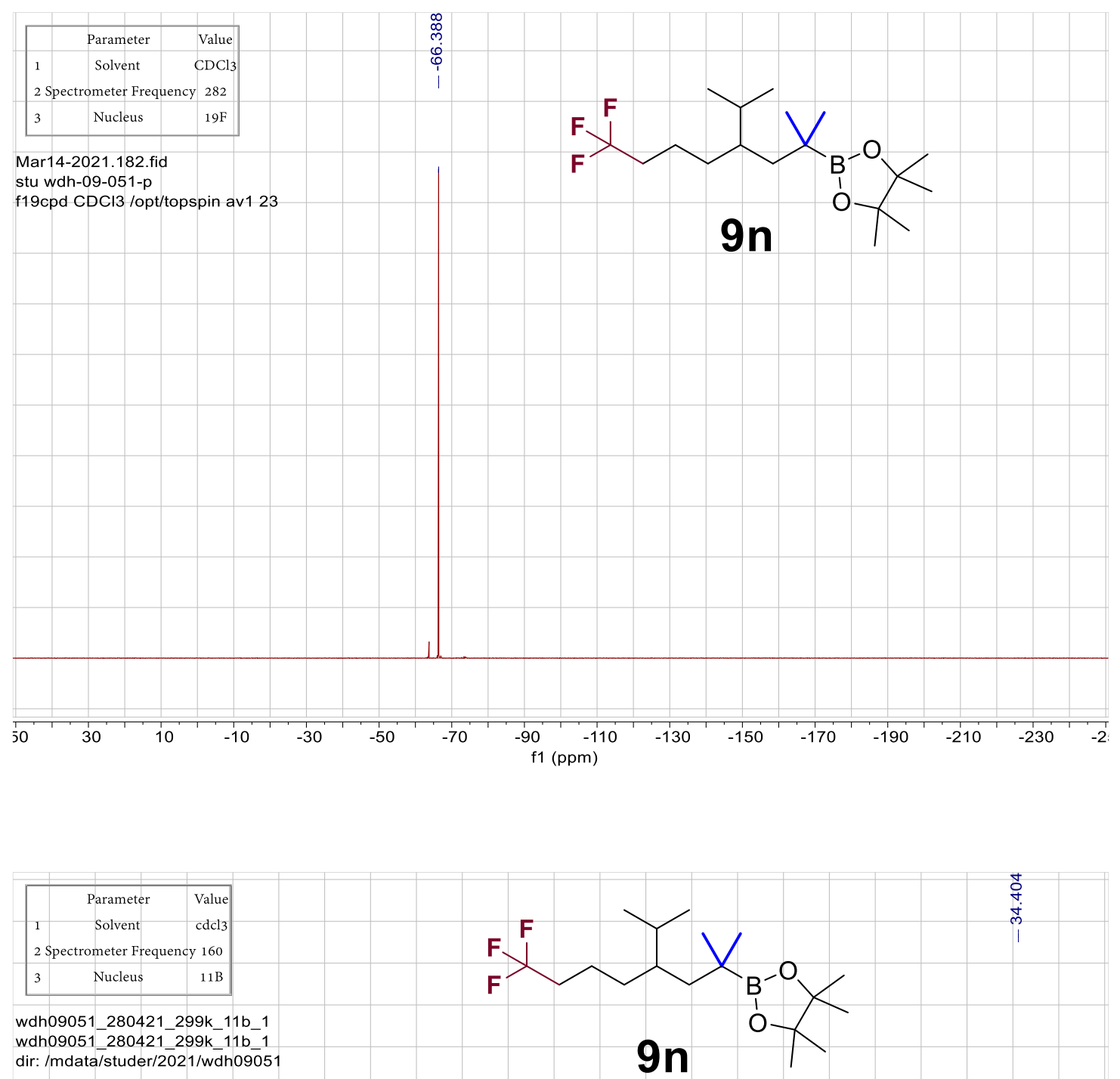

v500, probe: hfx, temp: $299 \mathrm{~K}$

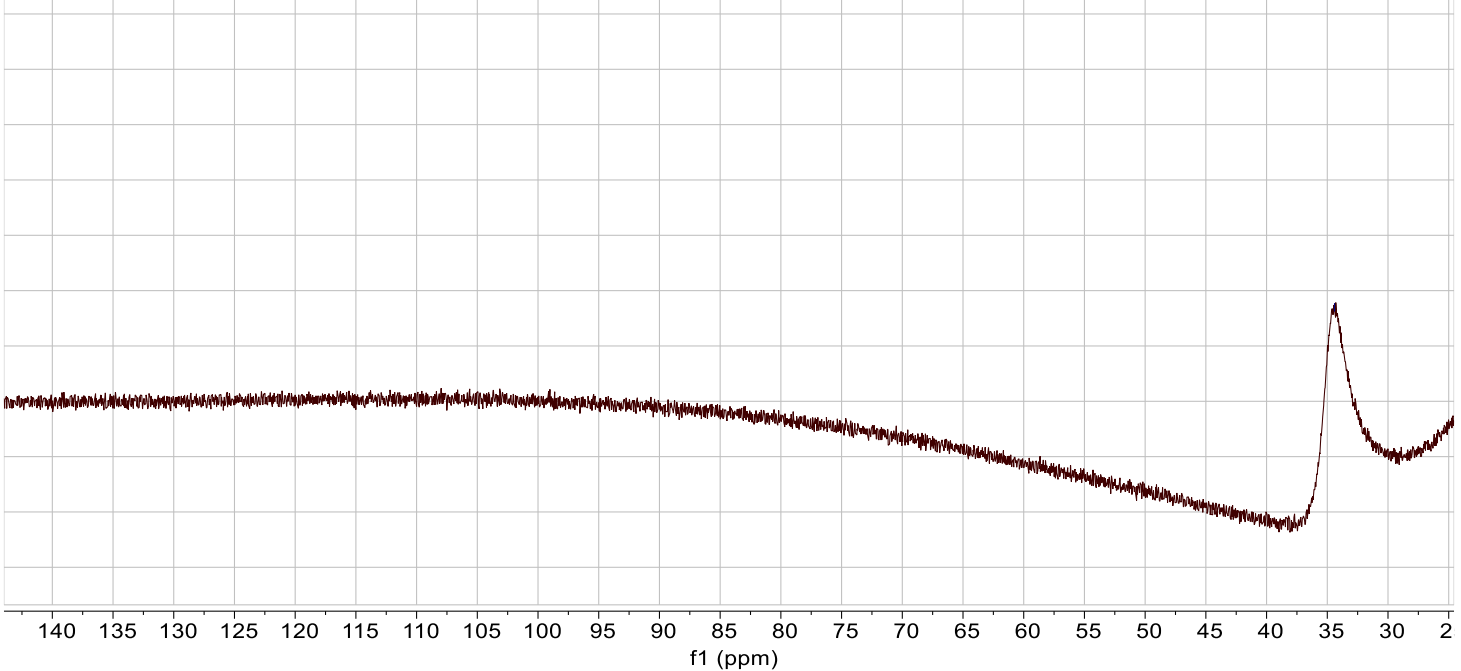

